\title{
Cis Selective RCM Study to the 14-Membered Cyclic Subunit of Bielschowskysin
}

\author{
Subramanian G. Gramani ${ }^{\dagger}{ }^{\ddagger}$, Ravi K. Sriramula ${ }^{\ddagger}$, Karthik Sekar ${ }^{\ddagger}$, Eugene G. Yang ${ }^{\ddagger}$, \\ Praveena Battu ${ }^{\ddagger}$, Aïcha Kopecky ${ }^{\ddagger}$, and Martin J. Lear ${ }^{\ddagger}$, ,*
}

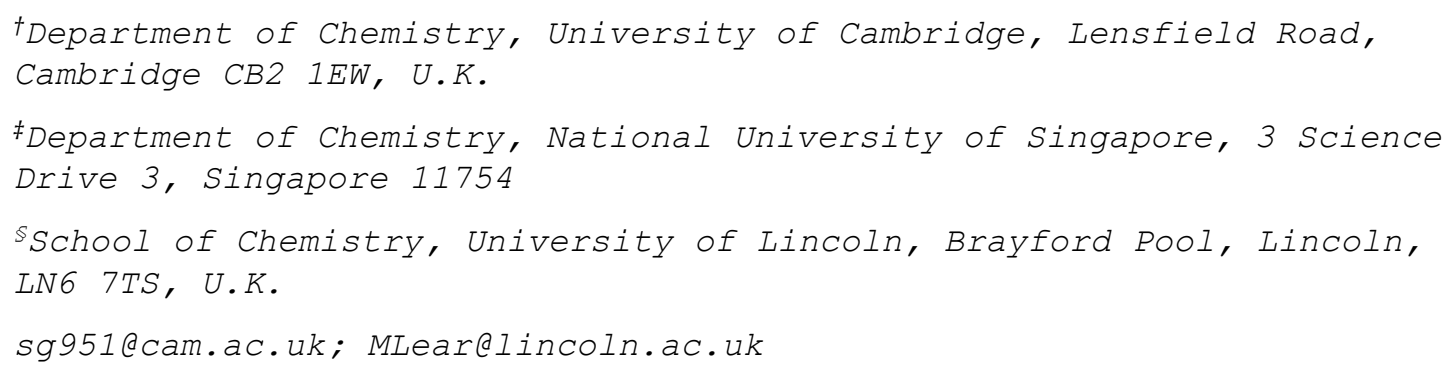




\section{Table of Contents}

\begin{tabular}{|c|c|}
\hline Compounds & Page no. \\
\hline DFT Macrocyclizations Studies & 3 \\
\hline DFT data including cartesian co-ordinates & 8 \\
\hline $\begin{array}{l}\text { (3aR,5S,6R,6aR)-5-((R)-2,2-dimethyl-1,3-dioxlan-4-yl)-6-(2-iodoethyl)-2,2- } \\
\text { dimethyltetrahydrofuro [2,3-d][1,3]dioxole (4) }\end{array}$ & 75 \\
\hline $\begin{array}{l}\text { (3aR,5S,6R,6aR)-6-(but-3-en-1-yl)-5-((R)-2,2-dimethyl-1,3-dioxolan-4-yl)-2,2- } \\
\text { dimethyltetrahydro furo[2,3-d][1,3] dioxole (6) }\end{array}$ & 76 \\
\hline $\begin{array}{l}\text { Methyl (E)-3-((3aR,5R,6R,6aR)-6-(but-3-en-1-yl)-2,2-dimethyltetrahydrofuro[2,3- } \\
\text { d][1,3]dioxol-5-yl)-2-methylacry late (8) }\end{array}$ & 77 \\
\hline $\begin{array}{l}(E)-3-((3 \mathrm{a} R, 5 R, 6 R, 6 \mathrm{a} R)-6-(\text { but-3-en-1-yl)-2,2-dimethyltetrahydrofuro[2,3- } d][1,3] \text { dioxol-5-yl)- } \\
\text { 2-methylprop-2-en-1-ol (9) }\end{array}$ & 78 \\
\hline $\begin{array}{l}(E)-3-((3 \mathrm{a} R, 5 R, 6 R, 6 \mathrm{a} R)-6-(\mathrm{But}-3 \text {-enyl)-2,2-dimethyltetrahydrofuro[2,3- } d][1,3] \text { dioxol -5-yl)-2- } \\
\text { methylacrylaldehyde }(\mathbf{1 0})\end{array}$ & 79 \\
\hline$(2 \mathrm{~S}, 4 \mathrm{~S})$-2-phenyl-4-vinyl-1,3-dioxane (13) & 80 \\
\hline (S)-3-(benzyloxy)pent-4-en-1-ol (13a) & 81 \\
\hline$(S)$-3-(benzyloxy)pent-4-enal (14) & 82 \\
\hline (S)-4-(benzyloxy)hex-5-en-2-one (15) & 84 \\
\hline (5S)-5-(benzyloxy)-3-methylhept-6-en-1-yn-3-ol (16) & 85 \\
\hline$(3 R, 5 S)$-5-(benzyloxy)-3-methylhept-6-en-1-yn-3-ol(16s) & 86 \\
\hline$((3 \mathrm{~S}, 5 \mathrm{~S})-5$-(benzyloxy)-3-methylhept-6-en-1-yn-3-yloxy)trimethylsilane (17) & 87 \\
\hline $\begin{array}{l}(3 R, 6 S, 8 S, E)-8 \text {-(benzyloxy)-1-((3a } R, 5 R, 6 R, 6 \mathrm{a} R)-6 \text {-(but-3-enyl)-2,2-dimethyltetrahydrofuro[2,3- } \\
\text { d] [1,3]dioxol-5-yl)-2,6-dimethyl-6-(trimethylsilyloxy)deca-1,9-dien-4-yn-3-ol (18a) }\end{array}$ & 88 \\
\hline $\begin{array}{l}(3 S, 6 S, 8 S, E)-8 \text {-(Benzyloxy)-1-((3a R,5R,6R,6aR)-6-(but-3-enyl)-2,2- } \\
\text { dimethyltetrahydrofuro[2,3- } d][1,3] \text { dioxol-5-yl)-2,6-dimethyl-6-(trimethylsilyloxy)deca-1,9- } \\
\text { dien-4-yn-3-ol (18b) }\end{array}$ & 88 \\
\hline $\begin{array}{l}((3 S, 5 S, 8 R, E)-3 \text {-(benzyloxy)-10-((3a } R, 5 R, 6 R, 6 \mathrm{a} R) \text {-6-(but-3-enyl)-2,2- } \\
\text { dimethyltetrahydrofuro[2,3- } d][1,3] \text { dioxol-5-yl)-8-methoxy-5,9-dimethyldeca-1,9-dien-6-yn-5- } \\
\text { yloxy)trimethylsilane (19) }\end{array}$ & 89 \\
\hline $\begin{array}{l}(4 S, 7 \mathrm{R})-4-((S)-2-(\text { benzyloxy)but-3-enyl)-7-( }(E)-1-((3 \mathrm{a} R, 5 R, 6 R, 6 \mathrm{a} R)-6 \text {-(but-3-enyl)-2,2- } \\
\text { dimethyl tetrahydrofuro[2,3- } d][1,3] \text { dioxol-5-yl)prop-1-en-2-yl)-9,9-diethyl-2,2,4-trimethyl-3,8- } \\
\text { dioxa-2,9-disilaundec-5-yne (20a) }\end{array}$ & 91 \\
\hline $\begin{array}{l}(4 S, 7 S)-4-((S)-2-(\text { benzyloxy)but-3-enyl)-7-((E)-1-((3a } R, 5 R, 6 R, 6 \mathrm{a} R)-6 \text {-(but-3-enyl)-2,2-dimethyl } \\
\text { tetrahydrofuro[2,3- } d][1,3] \text { dioxol-5-yl)prop-1-en-2-yl)-9,9-diethyl-2,2,4-trimethyl-3,8-dioxa-2,9- } \\
\text { disilaundec-5-yne (20b) }\end{array}$ & 92 \\
\hline $\begin{array}{l}(6 S, 8 S, E)-8 \text {-(benzyloxy)-1-((3a } R, 5 R, 6 R, 6 \mathrm{a} R)-6 \text {-(but-3-en-1-yl)-2,2-dimethyltetrahydrofuro[2,3- } \\
d][1,3] \text { dioxol-5-yl)-2,6-dimethyl-6-((trimethylsilyl)oxy)deca-1,9-dien-4-yn-3-one (21) }\end{array}$ & 92 \\
\hline${ }^{1} \mathrm{H}$ NMR $\left(500 \mathrm{MHz}, \mathrm{CDCl}_{3}\right)$ and ${ }^{13} \mathrm{C}\{1 \mathrm{H}\} \mathrm{NMR}\left(\mathrm{CDCl}_{3}, 125 \mathrm{MHz}\right.$ and DEPT) Spectrums of 22 & $93-94$ \\
\hline COSY Spectrum of $\mathbf{2 2}$ & 95 \\
\hline HMQC Spectrum of $\mathbf{2 2}$ & 96 \\
\hline NOESY Spectrum of 22 & 97 \\
\hline$(2 R, 4 S, 6 S)$-4-ethynyl-2-phenyl-6-vinyl-1,3-dioxane $(\mathbf{2 7 h})$ & 98 \\
\hline $\begin{array}{l}(E)-1-((3 \mathrm{a} R, 5 R, 6 R, 6 \mathrm{a} R)-6 \text {-(but-3-en-1-yl)-2,2-dimethyltetrahydrofuro[2,3-d][1,3]dioxol-5-yl)- } \\
\text { 2-methyl-5-((2R,4S,6S)-2-phenyl-6-vinyl-1,3-dioxan-4-yl)pent-1-en-4-yn-3-ol (28h) }\end{array}$ & 99 \\
\hline $\begin{array}{l}(E)-1-((3 \mathrm{a} R, 5 R, 6 R, 6 \mathrm{a} R)-6-(\text { but-3-en-1-yl)-2,2-dimethyltetrahydrofuro[2,3- } d][1,3] \text { dioxol-5-yl)- } \\
\text { 2-methyl-5-((2R,4S,6S)-2-phenyl-6-vinyl-1,3-dioxan-4-yl)pent-1-en-4-yn-3-one (31) }\end{array}$ & 100 \\
\hline$(2 S, 4 S, 6 S)$-4-ethynyl-4-methyl-2-phenyl-6-vinyl-1,3-dioxane (27) & 101 \\
\hline $\begin{array}{l}(E)-1-((3 \mathrm{a} R, 5 R, 6 R, 6 \mathrm{a} R)-6 \text {-(but-3-en-1-yl)-2,2-dimethyltetrahydrofuro[2,3- } d][1,3] \text { dioxol-5-yl)- } \\
\text { 2-methyl-5-((2R,4S,6S)-4-methyl-2-phenyl-6-vinyl-1,3-dioxan-4-yl)pent-1-en -4-yn-3-ol (28) }\end{array}$ & 102 \\
\hline $\begin{array}{l}(E)-1-((3 \mathrm{a} R, 5 R, 6 R, 6 \mathrm{a} R)-6 \text {-(but-3-en-1-yl)-2,2-dimethyltetrahydrofuro[2,3-d][1,3]dioxol-5-yl)- } \\
\text { 2-methyl-5-((2R,4S,6S)-4-methyl-2-phenyl-6-vinyl-1,3-dioxan-4-yl)pent-1-en -4-yn-3-one (29) }\end{array}$ & 103 \\
\hline${ }^{1} \mathrm{H} \mathrm{NMR}\left(500 \mathrm{MHz}, \mathrm{CDCl}_{3}\right)$ and ${ }^{13} \mathrm{C}\{1 \mathrm{H}\}$ NMR $\left(\mathrm{CDCl}_{3}, 125 \mathrm{MHz}\right)$ Spectrum of 30 & 104 \\
\hline
\end{tabular}


DFT Macrocyclizations Studies: Gaussian 09 software was used to analyze the stable energy and thermochemistry properties of the reaction [Frisch, M. J.; Trucks, G. W.; Schlegel, H. B.; Scuseria, G. E.; Robb, M. A.; Cheeseman, J. R.; Scalmani, G.; Barone, V.; Mennucci, B.; Petersson, G. A. Revision A 2009, 1]. Models were composed of the following basic key factors.

- Geometry optimization was used with 'opt' command

- Frequency for calculating zero-point vibrational energy thermochemistry properties was used with 'freq' command

- Computational method was HF/DFT under the hybrid functional method of B3LYP with the polarized basis set of 6-31G(d)

Energy and frequency values were calculated by changing the geometry of the C3-C4 double bond and the stereochemistry of the C5-methyl ether. From the results, we evaluated the conformation of the possible macrocycle precursor 2.19 and energies of formation of the 14-membered macrocycle 2.20 and the ethylene by product (Scheme 5).

The key issues to take into account are:

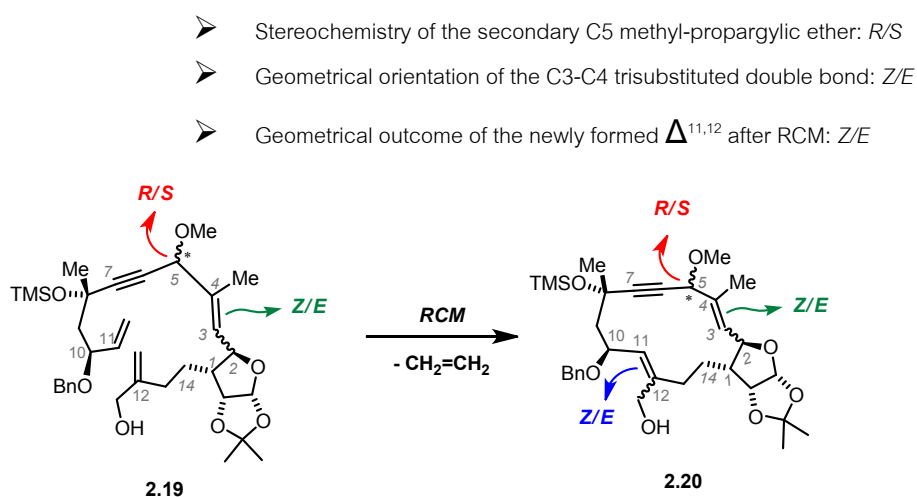

Scheme 5: RCM with alkyne di-olefin linear chain

By varying the above-mentioned factors, all diastereomers of the diene precursors (2.19a-2.19d) and macrocyclic products (2.20a-2.20d) were populated (Scheme 6). In order to predict the most feasible macrocycle formation from variable diene linear precursors in the synthesis of bielschowskysin, we selected the most established method DFT, B3LYP, 6-31G(d) basis set to calculate the frequency and minimum energy values.

Total energy of diene precursors: The total energy values at local minimum were obtained for each isomeric diene by DFT calculation. A comparative study indicates that the energy difference is diverse among the diene configurational isomers. The energy difference ranges from $\sim 0-9 \mathrm{kcal} / \mathrm{mol}$ (Table 1). The trisubstituted $E-\Delta^{3,4}$ olefin with $S_{\mathrm{C} 5} 2.19 \mathrm{a}$ was found to be the most stable $\left(-1368058.788 \mathrm{kcal} / \mathrm{mol}\right.$; entry 1 , Table 1). On the other hand, the $Z-\Delta^{3,4}$ olefin with $R_{\mathrm{C} 5} 2.19 \mathrm{~d}$ was highest in energy (-1368050.021 kcal/mol; entry 4, Table 1) by $8.76 \mathrm{kcal} / \mathrm{mol}$ than $2.19 \mathrm{a}$ (Fig 1\&2). 

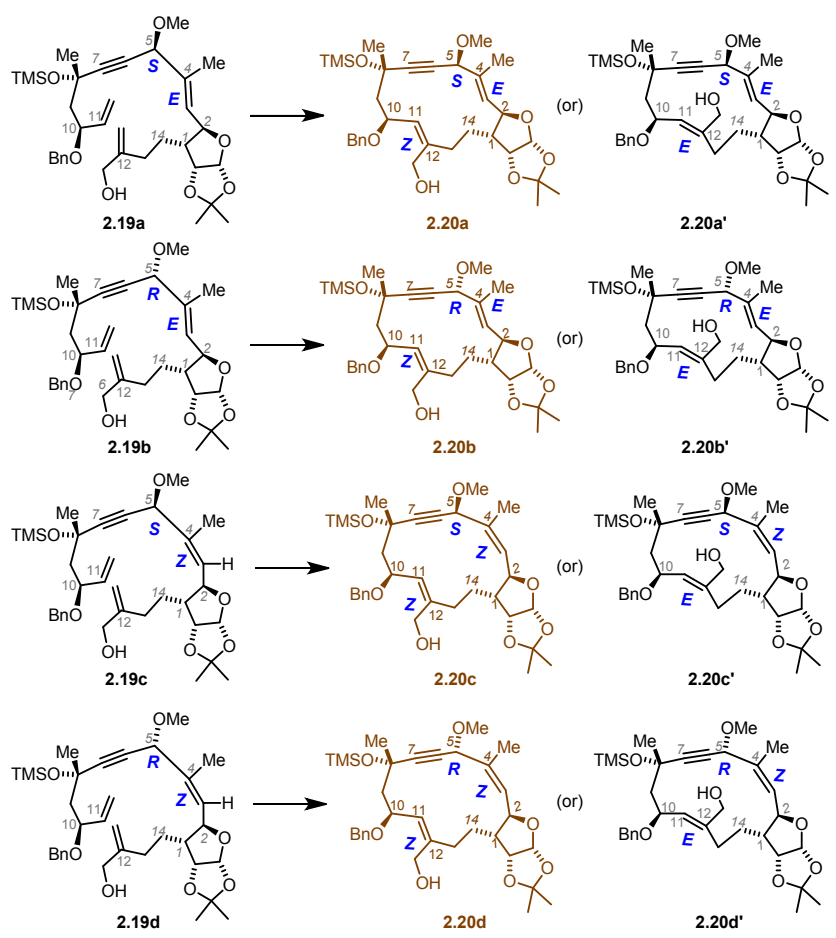

Scheme 6: RCM reaction

\begin{tabular}{|c|c|c|c|}
\hline Entry & Conformer & ZPE (Hartree) & $\begin{array}{l}\text { Total energy }(\mathrm{E}) \\
(\mathrm{kcal} / \mathrm{mol})\end{array}$ \\
\hline 1 & $2.19 a$ & 0.814361 & -1368058.788 \\
\hline 2 & $2.19 b$ & 0.813096 & -1368051.966 \\
\hline 3 & $2.19 c$ & 0.813505 & -1368051.573 \\
\hline 4 & $2.19 \mathrm{~d}$ & 0.81388 & -1368050.021 \\
\hline 5 & $2.20 a$ & 0.760287 & -1318767.511 \\
\hline 6 & 2.20a' & 0.760389 & -1318762.849 \\
\hline 7 & $2.20 \mathrm{~b}$ & 0.760366 & -1318767.043 \\
\hline 8 & $2.20 b^{\prime}$ & 0.760324 & -1318764.602 \\
\hline 9 & $2.20 \mathrm{c}$ & 0.760317 & -1318763.646 \\
\hline 10 & $2.20 c^{\prime}$ & 0.761006 & -1318762.724 \\
\hline 11 & $2.20 d$ & 0.760344 & -1318763.106 \\
\hline 12 & $2.20 d^{\prime}$ & 0.761104 & -1318763.659 \\
\hline 13 & Ethylene & 0.051226 & -49280.3234 \\
\hline
\end{tabular}

Table 1: Total energy values obtained from DFT, B3LYP, 6-31G(d) at 297K 


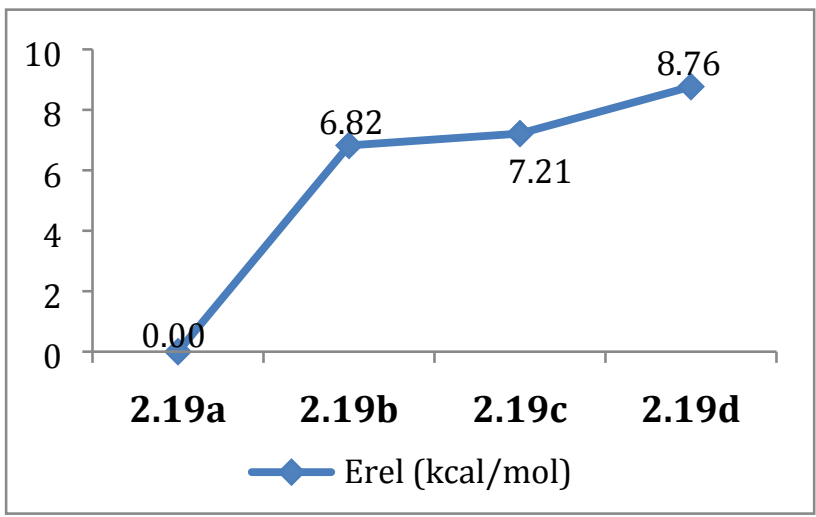

Figure 2: Relative energy of alkyne di-olefin linear chains

Total energy of desired RCM products: RCM can produce both Z and $E-\Delta^{11,12}$ olefins (Scheme 6). Hence, 4 diastereomeric precursors (2.19) could in principle produce 8 diastereomeric macrocycles. Among these 8 (2.20) macrocycles, the individual total energy value is the lowest $(-1318767.511 \mathrm{kcal} / \mathrm{mol})$ for a macrocycle in which a Z-configuration is at the newly generated $\Delta^{11,12}$ double bond $2.20 \mathrm{a}$ for a preexisting $E-\Delta^{3,4}$ double bond and $S_{\mathrm{C} 5}$ (entry 5 , table 1 and Fig 3 ). The same new $Z-\Delta^{11,12}$ double bond for preexisting $E-\Delta^{3,4}$ olefin configurations but $R_{\mathrm{C} 5}$ containing macrocycle $2.20 \mathrm{~b}$ is above by only $0.47 \mathrm{kcal} / \mathrm{mol}$ in energy (entry 7 , table 1 and Fig 3).The highest energy was observed for new $E-\Delta^{11,12}$, particularly with $S_{\mathrm{C} 5} 2.20 \mathrm{a}^{\prime}$ and $2.20 \mathrm{c}^{\prime}$ (entry 6 and entry 10 , table 1 and Fig 3 ). Both $Z-\Delta^{3,4}$ and $Z$ $\Delta^{11,12}$ double bonds with $R_{\mathrm{C} 5} 2.20 \mathrm{~d}$ are again close in energy to the above systems (entry 11 , table 1 and Fig 3 ). These high energy values could perhaps develop due to the strain imposed by restricted orientation of double bonds and other fused ring systems within the macrocycle. The overall energy difference ranges from $\sim 0-5 \mathrm{kcal} / \mathrm{mol}$ among all the possible RCM products (table 1; Fig 3).

Free energy change of RCM reactions: The alkyne linear chain precursor 2.19a which possess $S_{C 5}$ and $E-\Delta^{3,4}$ can give two possible macrocycles with a ' $Z$ ' 2.20 a or ' $E$ ' 2.20a' geometry at the nascent $\Delta^{11,12}$ olefin (Scheme 6). From the DFT calculation, formation of the new $\Delta^{11,12}$ double bond with a Z-geometry in the macrocycle 2.20a $\left(\Delta \mathrm{G}=2.06 \mathrm{kcal} / \mathrm{mol}\right.$; entry 2, table 2) is always more thermodynamically favorable than formation of an $E-\Delta^{11,12}$ in the macrocycle $2.20 \mathrm{a}$ ' $(\Delta \mathrm{G}=6.6 \mathrm{kcal} / \mathrm{mol}$, entry 1, table 2). This is substantiated from the less +ve $\Delta G$ values for Z-olefin formation over its $E$-olefin counterpart (table 2 and Fig 4 ). A negative free energy change for a chemical reaction indicates spontaneity of the reaction. In the context of macrocyclization, the macrocyclic product always occupies a higher energy values due to restricted degrees of freedom, and the strain developed in the rigid cyclic system. However, macrocycles can be formed from their lower energy precursor under techniques such as dilution, concentration, and temperature control to overcome both kinetic/thermodynamic barriers.

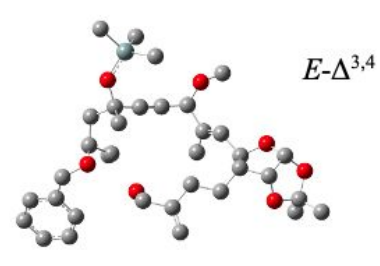

2.19a- $(5 S)$ $\mathrm{E}_{\text {rel }}=0.00 \mathrm{kcal} / \mathrm{mol}$

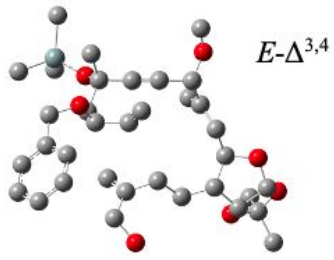

2.19b- $(5 R)$

$\mathrm{E}_{\text {rel }}=6.82 \mathrm{kcal} / \mathrm{mol}$ 


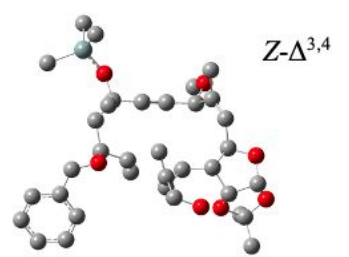

$$
\text { 2.19c- }(5 S)
$$

$\mathrm{E}_{\text {rel }}=7.21 \mathrm{kcal} / \mathrm{mol}$

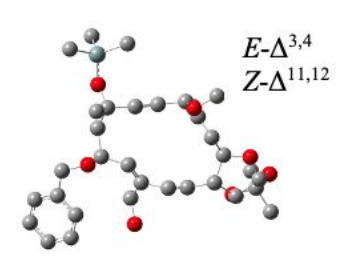

2.20a- $(5 S)$

$\mathrm{E}_{\mathrm{rel}}=0.00 \mathrm{kcal} / \mathrm{mol}$

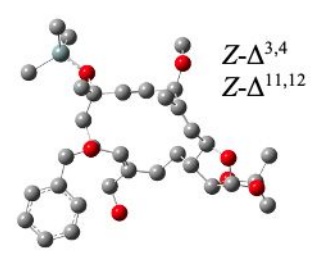

$$
2.20 \mathrm{c}-(5 S)
$$

$\mathrm{E}_{\text {rel }}=3.86 \mathrm{kcal} / \mathrm{mol}$
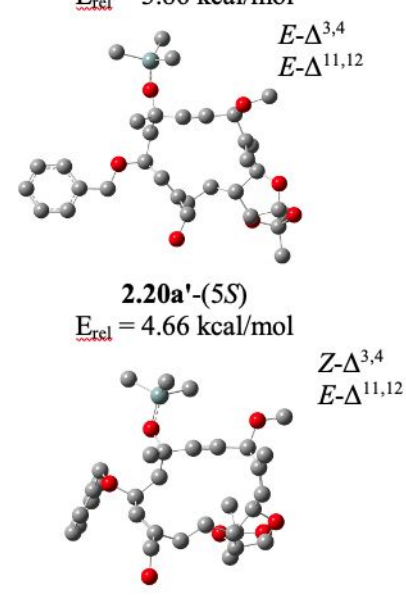

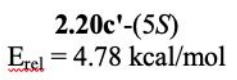

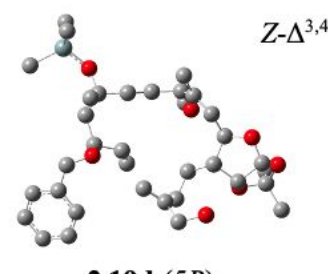

2.19d- $(5 R)$

$\mathrm{E}_{\mathrm{rel}}=8.76 \mathrm{kcal} / \mathrm{mol}$

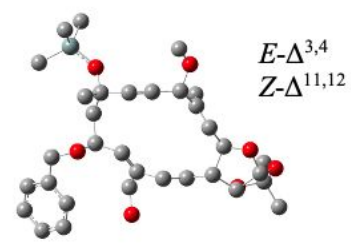

$2.20 \mathrm{~b}-(5 R)$

$\mathrm{E}_{\text {rel }}=0.47 \mathrm{kcal} / \mathrm{mol}$

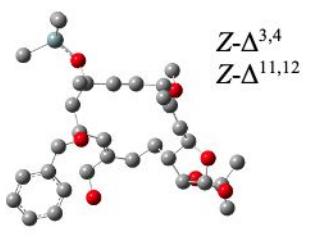

2.20d-( $5 R$ )

$\mathrm{E}_{\text {rel }}=4.40 \mathrm{kcal} / \mathrm{mol}$

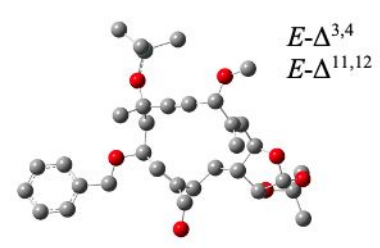

$2.20 b^{\prime}-(5 R)$

$\mathrm{E}_{\text {rel. }}=2.91 \mathrm{kcal} / \mathrm{mol}$

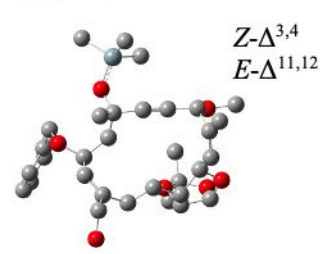

2.20d'-(5R)

$\mathrm{E}_{\text {rel }}=3.85 \mathrm{kcal} / \mathrm{mol}$

Figure 3: Geometry optimization with DFT, B3LYP, 6-31G(d) at 297K

\begin{tabular}{llll}
\hline & & $\Delta \mathrm{G}^{\dagger}$ & $\Delta \mathrm{H}$ \\
\hline Entry & Conformer & $(\mathrm{kcal} / \mathrm{mol})$ & $(\mathrm{kcal} / \mathrm{mol})$ \\
\hline 1 & $2.20 \mathrm{a}$ & 2.06 & 11.55 \\
\hline 3 & $2.20 \mathrm{a}^{\prime}$ & 6.6 & 16.21 \\
\hline & $2.20 \mathrm{~b}$ & -1.79 & 5.19 \\
\hline & & & 7.63 \\
\hline
\end{tabular}




\begin{tabular}{llcc}
5 & $2.20 c$ & -0.07 & 8.2 \\
6 & $2.20 c^{\prime}$ & 2.64 & 9.11 \\
7 & $2.20 d$ & -1.5 & 7.18 \\
8 & $2.20 d^{\prime}$ & -1.27 & 6.63 \\
\hline
\end{tabular}

Table 2: Free energy, enthalpy differences obtained from DFT, B3LYP, 6-31G(d) - 297K

The alkyne linear chain $2.19 \mathrm{~b}$ with $E-\Delta^{3,4}$ and R-configuration at C5 position can form either a $Z-\Delta^{11,12}$ macrocycle $2.20 \mathrm{~b}$ or $E-\Delta^{11,12}$ macrocycle $2.20 \mathrm{~b}$ after RCM (Scheme 2). These have a $\Delta \mathrm{G}$ of formation of $-1.79,0.31 \mathrm{kcal} / \mathrm{mol}$ respectively (entry 3, 4; table 2); and a $\Delta \mathrm{H}$ formation of $5.19,7.63 \mathrm{kcal} / \mathrm{mol}$ respectively. The ve $\Delta \mathrm{G}$ for $2.20 \mathrm{~b}$ confirms that the formation of the desired, $Z-\Delta^{11,12}$ macrocycle is relatively more feasible than for the other $E-\Delta^{11,12}$ isomer $2.20 \mathrm{~b}^{\prime}$ (Fig 4 ).

The two diastereomeric $Z-\Delta^{3,4}$ linear chains $2.19 \mathrm{c}, 2.19 \mathrm{~b}$ (differing at the C5-propargylic ether stereochemistry) vary only by $2.5 \mathrm{kcal} / \mathrm{mol}$. The $S$-isomer $2.19 \mathrm{c}$ with a $Z-\Delta^{3,4}$ double bond can produce two possible macrocycles $2.20 \mathrm{c}, 2.20 \mathrm{c}$ ' by eliminating ethylene gas as a byproduct during the RCM reaction (Scheme 2). Similar to previous cases, the $Z-\Delta^{11,12}$ macrocycle $2.20 \mathrm{c}$ is more feasible than $E-\Delta^{11,12}$ macrocycle $2.20 \mathrm{c}^{\prime}$ as the $\Delta \mathrm{G}$ values are $-0.07,2.64 \mathrm{kcal} / \mathrm{mol}$ for $2.20 \mathrm{c}, 2.20 \mathrm{c}$ respectively (entry 5,6 ; table 2).

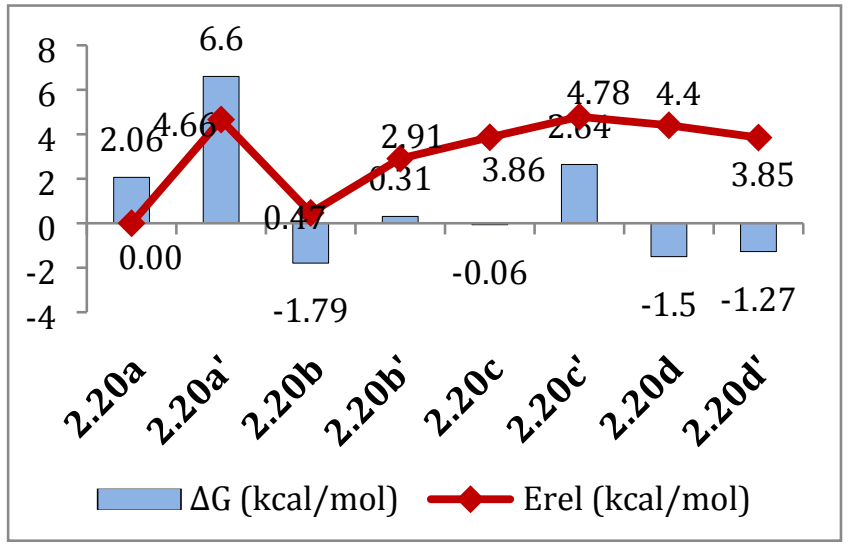

Figure 4: $\mathrm{E}_{\mathrm{rel}}$ and $\Delta \mathrm{G}$ differences for RCM products

On the other hand, the $R_{\mathrm{C} 5}$ linear precursor $2.19 \mathrm{~d}$ with $Z-\Delta^{3,4}$ upon RCM can produce $Z-\Delta^{11,12} 2.20 \mathrm{~d}$ or $E-\Delta^{11,12} 2.20 \mathrm{~d}^{\prime}$ macrocycles (Scheme 6). The $\Delta \mathrm{G}$ for the formation of $Z-\Delta^{11,12}$ and $E-\Delta^{11,12}$ macrocycles are -1.5, -1.27 kcal/mol respectively (entry 7, 8; table 2). From the DFT calculations, formation of both $Z-\Delta^{11,12}$ and $E-\Delta^{11,12}$ macrocycles $2.20 \mathrm{~d}, 2.20 \mathrm{~d}$ ' are feasible since the $\Delta \mathrm{G}$ values vary merely by $0.3 \mathrm{kcal} / \mathrm{mol}$ in their macrocyclic forms (Fig 4 ).

Summary: The above results can be summarized as follows

- The more stable $Z-\Delta^{3,4}$ olefin with $R_{C 5} 2.19 \mathrm{~b}$ will generate a newly formed $\Delta^{11,12}$ double bond with $Z$-geometry $2.20 \mathrm{~b}$ more readily, which is useful for butenolide construction.

- Macrocycles $2.20 \mathrm{a}, 2.20 \mathrm{~b}$ are lower in energy than the other isomers.

- $\Delta \mathrm{G}$ values for $2.20 \mathrm{~b}, 2.20 \mathrm{~d}$ are more negative than their competing isomers

- The linear precursors, $E-\Delta^{3,4} 2.19 \mathrm{~b}$ and $Z-\Delta^{3,4}$ olefin $2.19 \mathrm{~d}$ with $R_{\mathrm{C} 5}$ would more feasibly yield the desired $Z$-macrocycles $2.20 \mathrm{~b}, 2.20 \mathrm{~d}$.

- $R_{\mathrm{C} 5}$ is more advantageous than its $S$-counterpart, however, both are far apart in energy values.

- $\quad$ Pre-existing $\Delta^{3,4}$ olefin with Z/E configurations are almost equal in energy. 


\section{DFT study: Cartesian co-ordinates}

The cartesian co-ordinates obtained in the DFT study for each compound from '.out files' are shown as follows. 


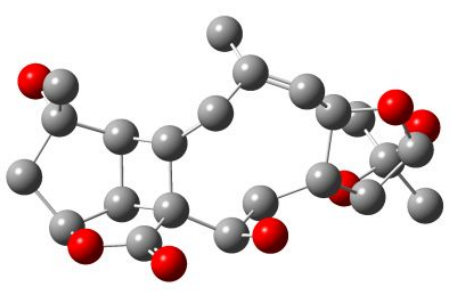

$$
\begin{array}{rr} 
& E-\Delta^{3,4} \\
\mathbf{2 . 1 0 a} & E-\Delta^{5,6} \\
\mathrm{E}_{\mathrm{rel}}=42.26 \mathrm{kcal} / \mathrm{mol}
\end{array}
$$

C

C

C

C

$\mathrm{H}$

$\mathrm{H}$

C

$\mathrm{H}$

O

O

C

C

$\mathrm{H}$

$\mathrm{H}$

$\mathrm{H}$

C

$\mathrm{H}$

$\mathrm{H}$

$\mathrm{H}$

C

$\mathrm{H}$

C

C

$\mathrm{H}$

C

$\mathrm{H}$

$\mathrm{H}$

$\mathrm{H}$

O

O

C

$\mathrm{H}$

$\mathrm{H}$

C

C

$\mathrm{H}$

C

O

C

$$
\begin{array}{ccc}
-2.43488 & 0.90508 & -0.55976 \\
-3.93318 & 1.3873 & -0.58378 \\
3.29077 & -1.13603 & -0.04426 \\
4.24018 & -0.46172 & 0.98717 \\
3.52645 & -2.19265 & -0.22847 \\
4.89008 & -1.13287 & 1.55514 \\
1.89918 & -0.91675 & 0.55262 \\
1.78441 & -1.683 & 1.3318 \\
3.46075 & -0.36544 & -1.22896 \\
5.03498 & 0.42201 & 0.21845 \\
4.74531 & 0.25832 & -1.17403 \\
5.80657 & -0.63654 & -1.82146 \\
5.59103 & -0.77683 & -2.88555 \\
6.79774 & -0.18441 & -1.71739 \\
5.82671 & -1.61931 & -1.33911 \\
4.64778 & 1.62839 & -1.82787 \\
5.60846 & 2.14714 & -1.75908 \\
4.37747 & 1.52652 & -2.88337 \\
3.88655 & 2.2268 & -1.32048 \\
-0.53366 & -1.87562 & 0.03563 \\
-0.56794 & -2.78643 & -0.57638 \\
-1.92528 & -1.20021 & -0.03124 \\
-2.36165 & -0.45527 & -1.31833 \\
-1.70934 & -0.52129 & -2.19096 \\
-4.5926 & 1.45445 & 0.79555 \\
-4.02809 & 2.11101 & 1.46464 \\
-5.61383 & 1.84843 & 0.70147 \\
-4.66085 & 0.46611 & 1.25884 \\
-3.89684 & 2.70004 & -1.16335 \\
3.43255 & 0.21544 & 1.92438 \\
-4.6481 & 0.37767 & -1.5321 \\
-5.69017 & 0.18167 & -1.25714 \\
-4.63707 & 0.81214 & -2.53893 \\
-1.96888 & 0.1754 & 0.68614 \\
-1.25245 & 0.39061 & 1.81164 \\
-1.82092 & 1.72517 & -0.93944 \\
-3.09123 & -2.11173 & 0.30947 \\
-3.14591 & -2.95358 & 1.17021 \\
-3.81681 & -0.90258 & -1.56594
\end{array}
$$


$\mathrm{O}$
$\mathrm{H}$
$\mathrm{H}$
$\mathrm{C}$
$\mathrm{H}$
$\mathrm{H}$
$\mathrm{H}$
$\mathrm{C}$
$\mathrm{C}$
$\mathrm{H}$
$\mathrm{C}$
$\mathrm{H}$
$\mathrm{C}$
$\mathrm{H}$
$\mathrm{H}$
$\mathrm{H}$
$\mathrm{O}$
$\mathrm{H}$

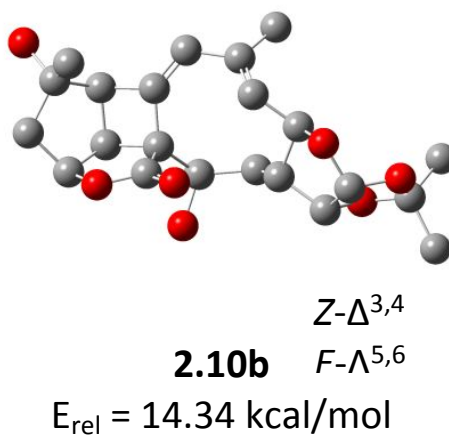

$\begin{array}{rrr}-4.16594 & -1.802 & -0.47979 \\ -3.95857 & -1.46865 & -2.49135 \\ -4.79693 & 3.06168 & -1.12387 \\ 0.68569 & -0.99444 & -0.42259 \\ 1.04752 & -1.37689 & -1.38267 \\ 0.35302 & 0.02607 & -0.6361 \\ -1.13326 & -0.50197 & 2.42475 \\ -0.09837 & 1.29064 & 1.89209 \\ 1.00839 & 0.59042 & 2.27312 \\ 0.86105 & -0.28901 & 2.90057 \\ 2.14744 & 0.43324 & 1.32628 \\ 2.20329 & 1.26171 & 0.60868 \\ -0.04477 & 2.59763 & 1.14117 \\ -0.12574 & 2.47417 & 0.05557 \\ -0.8779 & 3.24066 & 1.44667 \\ 0.88664 & 3.12869 & 1.35339 \\ -0.35047 & -2.33287 & 1.37811 \\ -0.25017 & -3.28761 & 1.38058\end{array}$

C

C

C

C

$\mathrm{H}$

$\mathrm{H}$

C

$\mathrm{H}$

O

O

C

C

$\mathrm{H}$

$\mathrm{H}$

$\mathrm{H}$

C

$\mathrm{H}$

$\mathrm{H}$

$\mathrm{H}$

C

$\begin{array}{ccc}-3.56101 & 0.59288 & -0.88217 \\ -4.76952 & 0.40573 & 0.10019 \\ 3.11493 & -1.1216 & 0.0237 \\ 3.63985 & -0.53812 & 1.37161 \\ 3.18398 & -2.21601 & -0.03155 \\ 3.82886 & -1.26105 & 2.16964 \\ 1.6847 & -0.59014 & -0.03797 \\ 1.08898 & -1.23806 & 0.61144 \\ 3.94141 & -0.51382 & -0.96474 \\ 4.85332 & 0.11048 & 1.02849 \\ 5.17059 & -0.13544 & -0.34478 \\ 6.19618 & -1.26996 & -0.4444 \\ 6.43565 & -1.47774 & -1.49237 \\ 7.11629 & -0.99669 & 0.08164 \\ 5.80207 & -2.18517 & 0.00943 \\ 5.65816 & 1.15697 & -0.98307 \\ 6.57663 & 1.49529 & -0.49436 \\ 5.86203 & 1.00109 & -2.04698 \\ 4.89475 & 1.93201 & -0.87606 \\ -0.46134 & -0.9585 & -1.56531\end{array}$




\begin{tabular}{|c|c|c|c|}
\hline $\mathrm{H}$ & -0.75958 & -0.6789 & -2.58385 \\
\hline $\mathrm{C}$ & -1.68065 & -0.58715 & -0.6598 \\
\hline $\mathrm{C}$ & -3.07646 & -0.81444 & -1.32855 \\
\hline $\mathrm{H}$ & -3.07805 & -1.03524 & -2.39747 \\
\hline C & -4.4863 & 0.8186 & 1.54661 \\
\hline $\mathrm{H}$ & -4.19264 & 1.87108 & 1.59698 \\
\hline $\mathrm{H}$ & -5.39085 & 0.68296 & 2.15495 \\
\hline $\mathrm{H}$ & -3.69513 & 0.21564 & 2.00079 \\
\hline 0 & -5.82346 & 1.2234 & -0.42983 \\
\hline 0 & 2.66766 & 0.36238 & 1.85493 \\
\hline $\mathrm{C}$ & -5.11848 & -1.10755 & -0.03218 \\
\hline $\mathrm{H}$ & -5.55063 & -1.54189 & 0.87612 \\
\hline $\mathrm{H}$ & -5.85231 & -1.20532 & -0.84138 \\
\hline C & -2.15548 & 0.86268 & -0.36192 \\
\hline $\mathrm{H}$ & -3.88112 & 1.27485 & -1.6751 \\
\hline $\mathrm{C}$ & -1.77422 & -1.4839 & 0.59634 \\
\hline 0 & -0.93637 & -1.68627 & 1.44163 \\
\hline C & -3.84221 & -1.82504 & -0.4622 \\
\hline O & -2.98983 & -2.08702 & 0.67905 \\
\hline $\mathrm{H}$ & -4.03273 & -2.79128 & -0.93809 \\
\hline $\mathrm{H}$ & -6.56479 & 1.18645 & 0.19593 \\
\hline C & 0.98971 & -0.42889 & -1.40275 \\
\hline $\mathrm{H}$ & 1.57427 & -0.94634 & -2.17354 \\
\hline $\mathrm{H}$ & 1.01241 & 0.62813 & -1.68943 \\
\hline $\mathrm{C}$ & -0.14212 & 2.15443 & 0.28487 \\
\hline C & 0.48151 & 1.2214 & 1.03485 \\
\hline $\mathrm{H}$ & -0.12177 & 0.52813 & 1.61352 \\
\hline $\mathrm{C}$ & 1.85587 & 0.74789 & 0.73769 \\
\hline $\mathrm{H}$ & 2.40955 & 1.46041 & 0.11283 \\
\hline $\mathrm{C}$ & 0.54422 & 3.28188 & -0.45862 \\
\hline $\mathrm{H}$ & 0.53253 & 3.13226 & -1.54673 \\
\hline $\mathrm{H}$ & 0.01012 & 4.22305 & -0.2695 \\
\hline $\mathrm{H}$ & 1.58066 & 3.42044 & -0.14162 \\
\hline $\mathrm{C}$ & -1.57972 & 2.01632 & 0.01664 \\
\hline $\mathrm{H}$ & -2.19559 & 2.91787 & -0.02991 \\
\hline 0 & -0.41372 & -2.3877 & -1.56397 \\
\hline $\mathrm{H}$ & -0.35551 & -2.70725 & -2.46735 \\
\hline
\end{tabular}

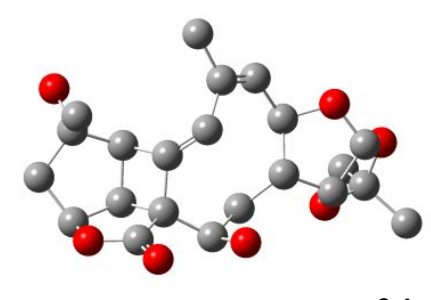

$$
\begin{array}{rr}
\mathbf{2 . 1 0 c} & \begin{array}{l}
Z-\Delta^{3,4} \\
Z-\Delta^{5,6}
\end{array} \\
E_{\text {rel }}=10.93 \mathrm{kcal} / \mathrm{mol}
\end{array}
$$

C

$\begin{array}{lll}-2.31755 & 1.07642 & -1.00011\end{array}$

C

$\begin{array}{lll}-3.772 & 1.58897 & -0.76552\end{array}$




\begin{tabular}{|c|c|c|c|}
\hline $\mathrm{C}$ & 3.24771 & -0.60864 & -0.50425 \\
\hline C & 4.1823 & -0.49254 & 0.68884 \\
\hline $\mathrm{H}$ & 3.51752 & -1.37023 & -1.20549 \\
\hline $\mathrm{H}$ & 4.63286 & -1.4274 & 0.95073 \\
\hline $\mathrm{C}$ & 1.92784 & -0.79779 & 0.14929 \\
\hline $\mathrm{H}$ & 1.85982 & -1.75529 & 0.62163 \\
\hline 0 & 3.37431 & 0.61747 & -1.18208 \\
\hline 0 & 5.13069 & 0.49888 & 0.26496 \\
\hline $\mathrm{C}$ & 4.82461 & 0.58294 & -1.13693 \\
\hline C & 5.34952 & -0.6558 & -1.88619 \\
\hline $\mathrm{H}$ & 5.07168 & -0.5929 & -2.91752 \\
\hline $\mathrm{H}$ & 6.41576 & -0.69585 & -1.80597 \\
\hline $\mathrm{H}$ & 4.92612 & -1.53902 & -1.45595 \\
\hline $\mathrm{C}$ & 5.44828 & 1.84027 & -1.77061 \\
\hline $\mathrm{H}$ & 6.51376 & 1.79103 & -1.68732 \\
\hline $\mathrm{H}$ & 5.17339 & 1.89303 & -2.80334 \\
\hline $\mathrm{H}$ & 5.09048 & 2.71067 & -1.26139 \\
\hline C & -0.43364 & -1.62429 & -0.20256 \\
\hline $\mathrm{H}$ & -0.32013 & -2.61603 & -0.58816 \\
\hline C & -1.79236 & -1.02235 & -0.45243 \\
\hline C & -2.4558 & -0.36469 & -1.66241 \\
\hline $\mathrm{H}$ & -2.28827 & -0.5502 & -2.7028 \\
\hline $\mathrm{C}$ & -4.06996 & 1.56875 & 0.74479 \\
\hline $\mathrm{H}$ & -3.41263 & 2.24713 & 1.24702 \\
\hline $\mathrm{H}$ & -5.08429 & 1.86536 & 0.91196 \\
\hline $\mathrm{H}$ & -3.92029 & 0.57982 & 1.12447 \\
\hline 0 & -3.99813 & 2.91388 & -1.25399 \\
\hline 0 & 3.37662 & -0.03888 & 1.83014 \\
\hline C & -4.64058 & 0.58765 & -1.58315 \\
\hline $\mathrm{H}$ & -5.67658 & 0.61548 & -1.31781 \\
\hline $\mathrm{H}$ & -4.55774 & 0.78819 & -2.63097 \\
\hline $\mathrm{C}$ & -1.56282 & 0.25485 & 0.07628 \\
\hline C & -0.73191 & 0.254 & 1.09869 \\
\hline $\mathrm{H}$ & -1.67855 & 1.8745 & -1.3157 \\
\hline $\mathrm{C}$ & -2.90058 & -1.84411 & 0.15503 \\
\hline 0 & -2.807 & -3.01404 & 0.60871 \\
\hline $\mathrm{C}$ & -3.94882 & -0.70197 & -1.28627 \\
\hline 0 & -4.06509 & -1.04931 & 0.10674 \\
\hline $\mathrm{H}$ & -4.3121 & -1.5491 & -1.82973 \\
\hline $\mathrm{H}$ & -4.916 & 3.15612 & -1.11035 \\
\hline C & 0.68282 & -0.71524 & -0.78896 \\
\hline $\mathrm{H}$ & 0.85471 & -1.09427 & -1.77448 \\
\hline $\mathrm{H}$ & 0.43206 & 0.32363 & -0.84116 \\
\hline $\mathrm{H}$ & -0.33999 & -0.71873 & 1.31196 \\
\hline $\mathrm{C}$ & 2.18304 & 0.45712 & 1.17787 \\
\hline $\mathrm{H}$ & 2.40833 & 1.30078 & 0.56005 \\
\hline C & -1.00061 & 2.47968 & 2.49632 \\
\hline $\mathrm{H}$ & -0.93627 & 3.29479 & 1.80654 \\
\hline $\mathrm{H}$ & -2.02402 & 2.18882 & 2.61042 \\
\hline $\mathrm{H}$ & -0.6085 & 2.78316 & 3.44479 \\
\hline 0 & -0.31676 & -1.64346 & 1.22242 \\
\hline
\end{tabular}




$\begin{array}{lrrl}\mathrm{H} & -1.00879 & -2.19656 & 1.5931 \\ \mathrm{C} & -0.18406 & 1.28776 & 1.96168 \\ \mathrm{C} & 1.13972 & 1.09293 & 2.23869 \\ \mathrm{H} & 1.52009 & 1.44067 & 3.17644\end{array}$

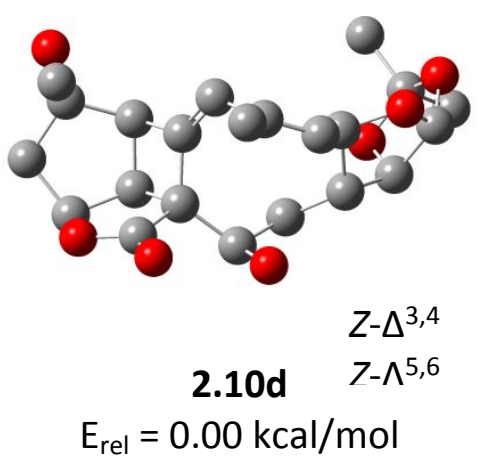

C

C

C

C

$\mathrm{H}$

$\mathrm{H}$

C

$\mathrm{H}$

O

O

C

C

$\mathrm{H}$

$\mathrm{H}$

$\mathrm{H}$

C

$\mathrm{H}$

$\mathrm{H}$

$\mathrm{H}$

C

$\mathrm{H}$

$\mathrm{H}$

C

$\mathrm{H}$

C

C

$\mathrm{H}$

C

$\mathrm{H}$

$\mathrm{H}$

$\mathrm{H}$

O

C

$\mathrm{H}$
$2.06915-1.33267$

$3.52098-1.62329$

$-3.38502$

$-4.12798$

$-3.89976$

$-4.939$

$-1.9934$

$-2.06813$

$-3.30678$

$-4.66734$

$-4.3596$

$-5.59722$

$-5.37675$

$-6.42239$

$-5.92046$

$-3.83917$

$-4.61356$

$-3.5525$

$-2.96731$

$-0.89241$

$-0.76981$

$-1.26233$

0.46392

0.78331

1.62113

2.12715

1.5492

4.11375

3.49011

5.11588

4.21726

3.42546

$-1.84599$

$-1.57781$
0.29754

0.90496

0.45532

1. 60204

0.94098

1.91477

$-1.09211$

$-0.20486$

$-1.41843$

$-1.8995$

$-2.82408$

$-2.08517$

$-1.1411$

$-2.44739$

$-2.68558$

$-3.367$

$-2.04566$

0.1046

$-0.82705$

$-0.19734$

0.83155

0.75212

$-1.15644$

$-1.84884$

$-0.53448$

$-0.38124$

$-0.83549$

0.41963

$-2.84985$

1. 21019

0.26996
0.30738
0.41816

0.93277

$-1.07513$

0.13843

$-2.03236$

$-0.09137$

$-1.02298$

$-1.52842$

$-0.76886$

0.83198

0.13945

$-0.62395$

$-1.16716$

0.07066

$-1.34454$

1.13304

1.86821

0.61339

1.65591

$-1.69184$

$-1.12945$

$-2.67929$

$-1.86904$

$-2.91684$

$-1.00837$

$-1.13109$

$-1.74549$

1.83079

2.71643

2.16517

1.30669

1.67267

0.49537

0.99546 


$\begin{array}{ccc}-3.17608 & 1.61252 & 0.89217 \\ -0.90617 & 2.30643 & 0.89699 \\ -1.31458 & 3.31189 & 0.79984 \\ 4.34513 & -1.82598 & -0.37561 \\ 5.39438 & -1.52358 & -0.28628 \\ 4.31636 & -2.89521 & -0.61823 \\ 1.46676 & 0.06824 & 0.50343 \\ 0.94961 & 0.78788 & 1.50165 \\ 0.99878 & 0.37558 & 2.5129 \\ 1.41226 & -2.12275 & 0.79276 \\ 2.88561 & 1.14387 & -1.25821 \\ 2.97417 & 2.34617 & -1.26919 \\ 3.62243 & -1.07108 & -1.48782 \\ 3.96578 & 0.33957 & -1.45996 \\ 3.87763 & -1.43427 & -2.48747 \\ 4.29993 & -3.02951 & 2.05428 \\ 0.34162 & 2.14048 & 1.35784 \\ 1.21771 & 3.30384 & 1.75802 \\ 1.61472 & 3.16664 & 2.77294 \\ 2.07705 & 3.38052 & 1.08067 \\ 0.66578 & 4.2489 & 1.73007 \\ 0.3309 & 2.23147 & -1.60934 \\ 0.29912 & 2.71006 & -2.44093\end{array}$

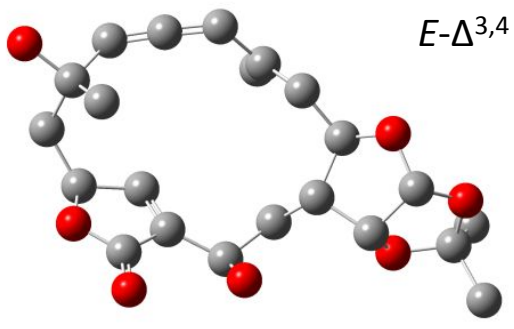

$$
\begin{gathered}
2.11 \mathrm{a}-(6 \mathrm{~S}) \\
\mathrm{E}_{\mathrm{rel}}=7.58 \mathrm{kcal} / \mathrm{mol}
\end{gathered}
$$

C

C

C

C

$\mathrm{H}$

$\mathrm{H}$

C

$\mathrm{H}$

O

0

C

C

$\mathrm{H}$

$\mathrm{H}$

$$
\begin{array}{rrr}
-2.57917 & -1.78728 & 0.38336 \\
-3.41281 & -0.50058 & 0.26988 \\
4.88506 & 0.71545 & -0.78028 \\
5.47032 & -0.65613 & -1.22155 \\
5.27638 & 1.56566 & -1.35391 \\
6.03239 & -0.6639 & -2.15939 \\
3.37608 & 0.51892 & -0.93209 \\
3.15485 & 0.6658 & -1.99881 \\
5.24671 & 0.82096 & 0.59265 \\
6.32517 & -1.03984 & -0.16029 \\
6.4012 & 0.00806 & 0.81239 \\
7.68159 & 0.82084 & 0.59782 \\
7.74547 & 1.63381 & 1.32815 \\
8.56053 & 0.17774 & 0.7058
\end{array}
$$


$\mathrm{H}$

C

$\mathrm{H}$

H

$\mathrm{H}$

C

H

$\mathrm{H}$

C

$\mathrm{H}$

C

C

$\mathrm{H}$

C

H

H

$\mathrm{H}$

C

$\mathrm{H}$

O

C

$\mathrm{H}$

C

C

$\mathrm{H}$

$\mathrm{H}$

H

C

$\mathrm{H}$

H

C

C

H

$\mathrm{H}$

C

O

C

0

H

O

H

o

$\mathrm{H}$

$\begin{array}{ccc}7.69733 & 1.25534 & -0.40705 \\ 6.31142 & -0.59569 & 2.20585 \\ 7.16464 & -1.25639 & 2.38538 \\ 6.31332 & 0.19508 & 2.9623 \\ 5.39016 & -1.17683 & 2.299 \\ 2.43977 & 1.40208 & -0.08626 \\ 2.23195 & 0.89227 & 0.86101 \\ 2.94203 & 2.33612 & 0.18804 \\ 1.12098 & 1.72246 & -0.83363 \\ 1.2187 & 2.65914 & -1.39378 \\ -0.13035 & 1.80503 & -0.0082 \\ -0.58507 & 1.05733 & 1.0062 \\ -0.06481 & 0.25861 & 1.51837 \\ -3.2582 & 0.13702 & -1.11448 \\ -3.61325 & -0.56183 & -1.87652 \\ -3.82049 & 1.07145 & -1.18921 \\ -2.20875 & 0.35432 & -1.32634 \\ 3.26684 & -1.00304 & -0.65068 \\ 3.43725 & -1.16224 & 0.4205 \\ 4.38374 & -1.53981 & -1.38678 \\ 1.97305 & -1.60382 & -1.08688 \\ 1.7807 & -1.5466 & -2.15897 \\ 0.98737 & -2.04446 & -0.27991 \\ 1.10785 & -2.27018 & 1.21028 \\ 0.25258 & -1.84662 & 1.74686 \\ 1.0877 & -3.34883 & 1.41388 \\ 2.02447 & -1.86555 & 1.64214 \\ -3.11957 & 0.47867 & 1.45148 \\ -4.02502 & 1.07585 & 1.59904 \\ -2.99731 & -0.12015 & 2.36251 \\ -1.47819 & -2.08363 & -0.25712 \\ -0.34277 & -2.35019 & -0.86795 \\ -0.36361 & -2.75754 & -1.88008 \\ -3.03036 & -2.52575 & 1.04785 \\ -1.20133 & 2.78268 & -0.35302 \\ -1.19902 & 3.6422 & -1.20108 \\ -1.96537 & 1.49927 & 1.40223 \\ -2.27117 & 2.56608 & 0.47985 \\ -1.93192 & 1.95827 & 2.4021 \\ 0.88903 & 0.71191 & -1.81846 \\ 0.92099 & 1.10436 & -2.694 \\ -4.74756 & -0.96562 & 0.48688 \\ -5.30756 & -0.68277 & -0.23975 \\ & & \end{array}$




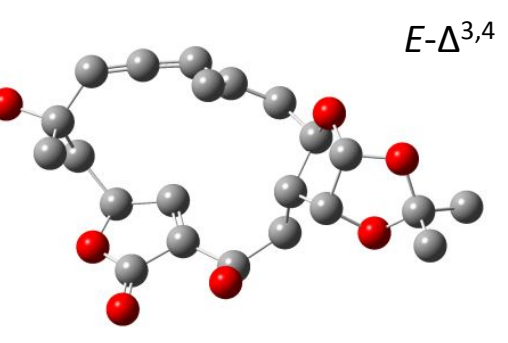

2.11b- $(6 R)$

$\mathrm{E}_{\mathrm{rel}}=8.24 \mathrm{kcal} / \mathrm{mol}$

C

C

C

C

$\mathrm{H}$

$\mathrm{H}$

O

O

C

C

$\mathrm{H}$

$\mathrm{H}$

$\mathrm{H}$

C

$\mathrm{H}$

$\mathrm{H}$

$\mathrm{H}$

C

$\mathrm{H}$

$\mathrm{H}$

C

$\mathrm{H}$

C

C

$\mathrm{H}$

C

$\mathrm{H}$

$\mathrm{H}$

$\mathrm{H}$

O

C

$\mathrm{H}$

C

$\mathrm{H}$

$\mathrm{H}$

C

C

$\mathrm{H}$

$\mathrm{H}$
0 .

1.53613

$-0.8253$

$-2.34327$

$-0.2207$

$-2.62226$

$-0.75472$

$-2.94784$

$-1.94011$

$-1.72868$

$-0.94541$

$-2.65589$

$-1.4294$

$-2.33262$

$-3.26376$

$-1.54764$

$-2.47923$

0.7135

0.4774

0.81775

2.0573

2. 88411

2. 3576

1.72088

0.75584

2.02175

1. 59215

3.11233

1.70346

3.75774

2. 61672

2. 98498

2. 14636

3.03804

1. 44905

$-0.78232$

$-1.51964$

$-0.42702$

$-1.87287$
0 .

0 .

7.28197

7.13715

7.49469

7.12113

8.36252

8.24958

9.14987

10.27279

10.95676

10.83721

9.86081

9.66554

10.2363

10.31539

8.8233

5.99355

5.38142

7.01762

5.52635

6.03618

4.05401

3.02136

3.0331

0.87645

0.51136

0.86832

1. 91329

2.20658

1.8103

1.70728

0.39176

$-0.22851$

0.07295

0.78481

1.67389

$-0.73314$

1.47328
0 .

0 .

0 .

0.30621

0.89095

1.36385

$-0.92293$

$-0.32662$

$-0.79321$

0.22695

$-0.11489$

0.36646

1.19639

$-2.16926$

$-2.10624$

$-2.56793$

$-2.85045$

$-1.66861$

$-2.54794$

$-2.0379$

$-1.07515$

$-1.58942$

$-1.16055$

$-1.73264$

$-2.22204$

1. 16648

2.10462

1. 24739

1.02575

$-0.95784$

$-1.74794$

$-2.78244$

$-1.38208$

$-1.50274$

$-2.16507$

$-0.69006$

$-1.32345$

0.68504

$-2.33613$ 


$\begin{array}{llc}-1.76556 & 3.02007 & -0.75937 \\ -1.73493 & 3.13606 & 0.74606 \\ -2.38878 & 2.37492 & 1.18747 \\ -0.72873 & 2.94491 & 1.1392 \\ -2.07447 & 4.11547 & 1.07876 \\ -1.9106 & 4.05787 & -1.60856 \\ -1.944 & 3.8373 & -2.67503 \\ -0.46476 & 5.94987 & -0.67772 \\ -0.23833 & 5.2348 & 0.12134 \\ -1.83417 & 5.52361 & -1.26983 \\ -2.03564 & 6.11096 & -2.17721 \\ -2.77268 & 5.91653 & -0.24665 \\ 3.65531 & 3.5392 & -0.63616 \\ 4.51486 & 4.1312 & -0.02917 \\ 2.18147 & 5.94696 & 0.28594 \\ 2.8611 & 6.62175 & 0.35209 \\ 1.86805 & -1.3739 & 0.21712 \\ 1.82429 & -1.56965 & 1.15593\end{array}$

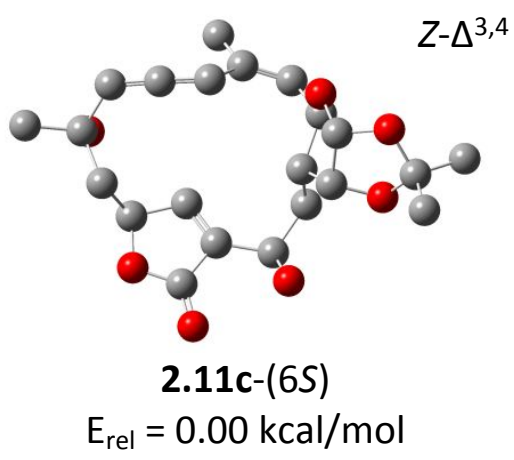

C
C
H
C
H
C
C
H
C
H
C
H
O
C
H
C
H
C

$\begin{array}{llr}-0.9913 & -1.57413 & -1.52468 \\ -2.15069 & -1.06682 & -1.85615 \\ -2.85266 & -1.65575 & -2.44779 \\ 0.19932 & -1.98817 & -1.14376 \\ 1.06113 & -1.78469 & -1.77792 \\ 0.4666 & -2.71417 & 0.11886 \\ 1.64073 & -2.6195 & 0.77402 \\ 1.74655 & -3.20407 & 1.68603 \\ 2.81376 & -1.72771 & 0.46534 \\ 3.63032 & -1.97396 & 1.15765 \\ 2.54183 & -0.2046 & 0.52684 \\ 1.67403 & -0.02201 & -0.11628 \\ 3.29981 & -1.90149 & -0.88924 \\ 3.76447 & 0.34407 & -0.21139 \\ 3.59457 & 1.30629 & -0.71077 \\ 4.13749 & -0.81177 & -1.18708 \\ 4.02731 & -0.59759 & -2.25418 \\ 2.25424 & 0.3732 & 1.91282\end{array}$




$\begin{array}{lcr}1.44414 & -0.21392 & 2.36512 \\ 3.13446 & 0.25218 & 2.55377 \\ 4.89057 & 0.42986 & 0.66534 \\ 5.49888 & -1.09905 & -0.89536 \\ 5.98767 & -0.06099 & -0.06958 \\ 1.85743 & 1.86585 & 1.89604 \\ 1.62498 & 2.17755 & 2.92315 \\ 0.69982 & 2.19414 & 0.99223 \\ -0.5059 & 1.62934 & 0.84072 \\ -0.92582 & 0.79096 & 1.382 \\ -1.24788 & 2.28316 & -0.29246 \\ -2.18595 & 2.74312 & 0.03658 \\ -1.47762 & 1.37716 & -1.51691 \\ -1.67784 & 2.03982 & -2.36516 \\ -0.529 & 0.87879 & -1.73069 \\ -2.63172 & 0.32937 & -1.40893 \\ -3.80135 & 0.7681 & -2.30417 \\ -4.6307 & 0.05376 & -2.25834 \\ -4.17146 & 1.7501 & -1.99188 \\ -3.47735 & 0.83745 & -3.34845 \\ -0.36544 & 3.34549 & -0.72112 \\ -0.649 & -3.57741 & 0.65808 \\ -1.55437 & -2.98056 & 0.82636 \\ -0.91897 & -4.36411 & -0.05746 \\ -0.36898 & -4.05023 & 1.60391 \\ 0.80888 & 3.28508 & -0.01722 \\ 1.74348 & 4.01839 & -0.2413 \\ 2.96823 & 2.66507 & 1.48099 \\ 3.27271 & 3.19842 & 2.21884 \\ 7.03226 & -0.63236 & 0.90713 \\ 7.87348 & -0.9978 & 0.35603 \\ 7.35234 & 0.13764 & 1.57761 \\ 6.59676 & -1.43391 & 1.46637 \\ 6.61348 & 1.05536 & -0.92616 \\ 6.77649 & 1.92152 & -0.31945 \\ 7.54749 & 0.71806 & -1.3246 \\ 5.95127 & 1.30214 & -1.72957 \\ -3.04568 & 0.27736 & -0.04115 \\ -4.00026 & 0.18488 & 0.00147\end{array}$

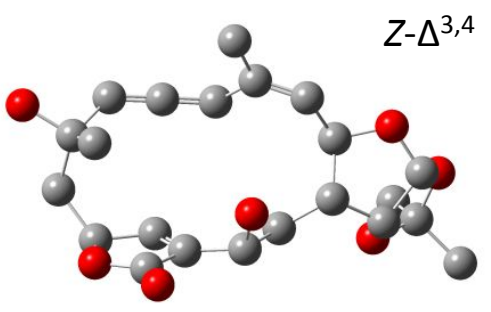

$$
\begin{gathered}
2.11 \mathrm{~d}-(6 R) \\
E_{\mathrm{rel}}=5.98 \mathrm{kcal} / \mathrm{mol}
\end{gathered}
$$




\begin{tabular}{|c|c|c|c|}
\hline $\mathrm{C}$ & -2.18825 & -1.3579 & 0.90796 \\
\hline $\mathrm{C}$ & -3.20101 & -0.26756 & 0.50368 \\
\hline C & 4.86235 & 0.57714 & -0.59219 \\
\hline C & 5.52442 & -0.81151 & -0.74515 \\
\hline $\mathrm{H}$ & 5.37207 & 1.37406 & -1.14957 \\
\hline $\mathrm{H}$ & 6.2563 & -0.91367 & -1.55124 \\
\hline C & 3.41136 & 0.36204 & -1.03664 \\
\hline $\mathrm{H}$ & 3.38875 & 0.44697 & -2.13205 \\
\hline 0 & 4.91521 & 0.82629 & 0.81026 \\
\hline O & 6.17108 & -1.04902 & 0.48992 \\
\hline C & 5.99684 & 0.07405 & 1.36168 \\
\hline C & 7.27939 & 0.91041 & 1.38103 \\
\hline $\mathrm{H}$ & 7.15853 & 1.78019 & 2.03462 \\
\hline $\mathrm{H}$ & 8.11858 & 0.30872 & 1.74355 \\
\hline $\mathrm{H}$ & 7.52135 & 1.26318 & 0.37316 \\
\hline $\mathrm{C}$ & 5.58931 & -0.41855 & 2.74253 \\
\hline $\mathrm{H}$ & 6.38498 & -1.03402 & 3.17253 \\
\hline $\mathrm{H}$ & 5.40202 & 0.42965 & 3.40825 \\
\hline $\mathrm{H}$ & 4.68046 & -1.02111 & 2.66575 \\
\hline C & 2.40924 & 1.34842 & -0.40893 \\
\hline $\mathrm{H}$ & 2.15082 & 0.98808 & 0.59235 \\
\hline $\mathrm{H}$ & 2.90006 & 2.31499 & -0.24597 \\
\hline C & 1.14435 & 1.56183 & -1.26263 \\
\hline $\mathrm{H}$ & 1.32648 & 2.31708 & -2.03631 \\
\hline $\mathrm{C}$ & -0.09801 & 1.93881 & -0.51058 \\
\hline $\mathrm{C}$ & -0.52321 & 1.63394 & 0.72207 \\
\hline $\mathrm{H}$ & 0.01909 & 1.11446 & 1.50224 \\
\hline $\mathrm{C}$ & -3.19591 & -0.0758 & -1.01881 \\
\hline $\mathrm{H}$ & -3.49863 & -1.01021 & -1.49971 \\
\hline $\mathrm{H}$ & -3.87412 & 0.7252 & -1.32666 \\
\hline $\mathrm{H}$ & -2.19457 & 0.17384 & -1.3764 \\
\hline $\mathrm{C}$ & 3.21958 & -1.14099 & -0.6693 \\
\hline $\mathrm{H}$ & 3.07404 & -1.20643 & 0.41875 \\
\hline 0 & 4.49099 & -1.72997 & -1.00959 \\
\hline 0 & -2.27157 & 2.74238 & -0.33108 \\
\hline C & -1.93628 & 2.10012 & 0.91609 \\
\hline $\mathrm{H}$ & -1.97981 & 2.88161 & 1.68897 \\
\hline C & -3.00647 & 1.05975 & 1.30388 \\
\hline $\mathrm{H}$ & -3.961 & 1.59396 & 1.26228 \\
\hline $\mathrm{H}$ & -2.84679 & 0.79371 & 2.3558 \\
\hline C & -0.94765 & -1.48644 & 0.51662 \\
\hline $\mathrm{C}$ & 0.32677 & -1.55952 & 0.18562 \\
\hline $\mathrm{H}$ & -2.61951 & -2.10759 & 1.57234 \\
\hline $\mathrm{H}$ & 1.03377 & -1.07292 & 0.857 \\
\hline C & 0.88974 & -2.14835 & -1.03855 \\
\hline $\mathrm{C}$ & 2.16875 & -1.92182 & -1.40623 \\
\hline $\mathrm{H}$ & 2.52424 & -2.34398 & -2.34457 \\
\hline $\mathrm{C}$ & -0.03564 & -2.96836 & -1.90383 \\
\hline $\mathrm{H}$ & -0.42218 & -3.83256 & -1.34946 \\
\hline $\mathrm{H}$ & -0.90757 & -2.38196 & -2.21932 \\
\hline
\end{tabular}


$\mathrm{H}$

C

O

O

$\mathrm{H}$

O

$\mathrm{H}$

$\begin{array}{rcr}0.47613 & -3.33351 & -2.79857 \\ -1.20347 & 2.66776 & -1.19273 \\ -1.22913 & 3.13871 & -2.30426 \\ 0.84134 & 0.35965 & -1.97526 \\ 0.89248 & 0.5253 & -2.91948 \\ -4.45139 & -0.79676 & 0.95244 \\ -5.16661 & -0.39697 & 0.45221\end{array}$

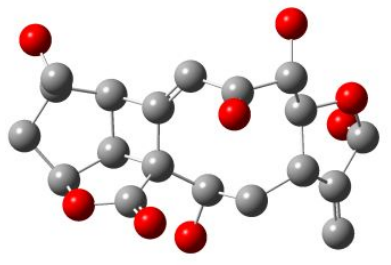

2.13a- $\left(Z-\Delta^{3,4}\right)$ $\mathrm{E}_{\mathrm{rel}}=0.00 \mathrm{kcal} / \mathrm{mol}$
C

C

C

$\mathrm{H}$

$\mathrm{H}$

C

$\mathrm{H}$

C

$\mathrm{H}$

$\mathrm{H}$

C

O

O

C

C

$\mathrm{H}$

C

C

C

$\mathrm{H}$

$\mathrm{H}$

O

C

$\mathrm{H}$

C

$\mathrm{H}$

O

$\mathrm{H}$

C

C

$\begin{array}{lrr}-1.96665 & -1.02214 & -1.09764 \\ -0.97602 & -0.82088 & 0.07677 \\ -2.1973 & 0.51353 & -1.20305 \\ -1.59167 & -1.55347 & -1.97217 \\ -1.97246 & 0.98696 & -2.16244 \\ -3.24796 & -1.61135 & -0.48425 \\ -3.47468 & -2.63197 & -0.80271 \\ -4.35074 & -0.61283 & -0.82691 \\ -4.70225 & -0.83122 & -1.84266 \\ -5.20484 & -0.68964 & -0.14524 \\ -1.75652 & -1.32294 & 1.30727 \\ -3.02476 & -1.66318 & 0.94721 \\ -1.37848 & -1.39422 & 2.44691 \\ -1.12126 & 0.6994 & -0.1378 \\ -0.46136 & 1.7924 & 0.25361 \\ -0.6964 & 2.7353 & -0.23903 \\ 0.63465 & 1.87069 & 1.28017 \\ 0.41154 & -1.46159 & -0.06793 \\ 1.37431 & -1.17172 & 1.11317 \\ 0.84448 & -0.66079 & 1.91726 \\ 1.65844 & -2.13659 & 1.54801 \\ 0.42799 & 1.76466 & 2.47386 \\ 2.65596 & -0.40158 & 0.75511 \\ 3.15402 & -0.13692 & 1.701 \\ 4.36543 & -0.10638 & -0.96229 \\ 5.4543 & -0.07204 & -0.82874 \\ 4.03396 & -0.35896 & -2.31353 \\ 4.41087 & 0.3715 & -2.83297 \\ 3.66517 & -1.14681 & -0.10371 \\ 3.9108 & -2.45473 & -0.15062\end{array}$




$\begin{array}{ccc}4.66242 & -2.86427 & -0.81965 \\ 3.38554 & -3.16579 & 0.483 \\ -4.20096 & 1.58082 & -1.95162 \\ -5.11529 & 1.82881 & -1.73943 \\ 2.53404 & 0.92013 & -0.0479 \\ 1.87508 & 0.79546 & -0.91622 \\ 3.87346 & 1.14316 & -0.51088 \\ -3.69982 & 0.80363 & -0.85425 \\ -3.91676 & 1.57052 & 0.45295 \\ -4.9898 & 1.75414 & 0.59913 \\ -3.55988 & 1.01264 & 1.32315 \\ -3.40464 & 2.53682 & 0.42568 \\ 0.84595 & -1.07675 & -1.00214 \\ 0.1499 & -2.85893 & -0.22781 \\ 0.98838 & -3.27934 & -0.47623 \\ 2.05227 & 2.13592 & 0.7337 \\ 2.71937 & 2.31545 & 1.58713 \\ 2.02555 & 3.24511 & -0.16332 \\ 2.00124 & 4.05444 & 0.37018\end{array}$

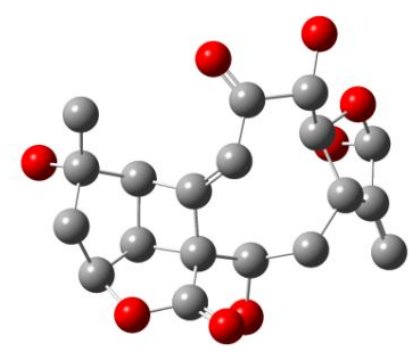

2.13b- $\left(E-\Delta^{3,4}\right)$ $\mathrm{E}_{\mathrm{rel}}=5.36 \mathrm{kcal} / \mathrm{mol}$

C

C

C

$\mathrm{H}$

$\mathrm{H}$

C

$\mathrm{H}$

C

$\mathrm{H}$

$\mathrm{H}$

C

0

O

C

C

C

$\mathrm{H}$

$\mathrm{H}$

$\begin{array}{lrr}-1.96665 & -1.02214 & -1.09764 \\ -0.97602 & -0.82088 & 0.07677 \\ -2.1973 & 0.51353 & -1.20305 \\ -1.59167 & -1.55347 & -1.97217 \\ -1.97246 & 0.98696 & -2.16244 \\ -3.24796 & -1.61135 & -0.48425 \\ -3.47468 & -2.63197 & -0.80271 \\ -4.35074 & -0.61283 & -0.82691 \\ -4.70225 & -0.83122 & -1.84266 \\ -5.20484 & -0.68964 & -0.14524 \\ -1.75652 & -1.32294 & 1.30727 \\ -3.02476 & -1.66318 & 0.94721 \\ -1.37848 & -1.39422 & 2.44691 \\ -1.12126 & 0.6994 & -0.1378 \\ 0.41154 & -1.46159 & -0.06793 \\ 1.37431 & -1.17172 & 1.11317 \\ 0.84448 & -0.66079 & 1.91726 \\ 1.65844 & -2.13659 & 1.54801\end{array}$




$\begin{array}{ccc}2.65596 & -0.40158 & 0.75511 \\ 3.15402 & -0.13692 & 1.701 \\ 4.36543 & -0.10638 & -0.96229 \\ 5.4543 & -0.07204 & -0.82874 \\ 4.03396 & -0.35896 & -2.31353 \\ 4.41087 & 0.3715 & -2.83297 \\ 3.66517 & -1.14681 & -0.10371 \\ 3.9108 & -2.45473 & -0.15062 \\ 4.66242 & -2.86427 & -0.81965 \\ 3.38554 & -3.16579 & 0.483 \\ -4.20096 & 1.58082 & -1.95162 \\ -5.11529 & 1.82881 & -1.73943 \\ 2.53404 & 0.92013 & -0.0479 \\ 1.87508 & 0.79546 & -0.91622 \\ 3.87346 & 1.14316 & -0.51088 \\ -3.69982 & 0.80363 & -0.85425 \\ -3.91676 & 1.57052 & 0.45295 \\ -4.9898 & 1.75414 & 0.59913 \\ -3.55988 & 1.01264 & 1.32315 \\ -3.40464 & 2.53682 & 0.42568 \\ 0.84595 & -1.07675 & -1.00214 \\ 0.1499 & -2.85893 & -0.22781 \\ 0.98838 & -3.27934 & -0.47623 \\ 2.05227 & 2.13592 & 0.7337 \\ 2.71937 & 2.31545 & 1.58713 \\ 2.02555 & 3.24511 & -0.16332 \\ 2.00124 & 4.05444 & 0.37018 \\ -0.45158 & 1.8086 & 0.25941 \\ -0.74896 & 2.32883 & 1.14588 \\ 0.74152 & 2.32207 & -0.56791 \\ 0.79066 & 2.11073 & -1.80746\end{array}$

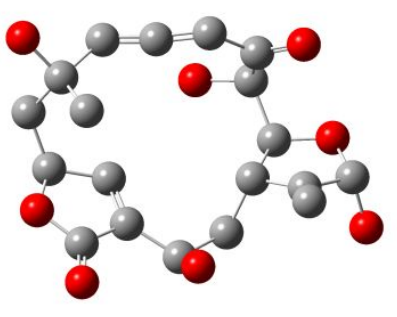

2.14a-(6S)

$E_{\text {rel }}=0.00 \mathrm{kcal} / \mathrm{mol}$

C

$\mathrm{O}$

C

$\mathrm{H}$

C

$\mathrm{H}$
2. 29727

3.39972

1. 49554

0.8825

3.00097

3.3689
2.54407

1.83631

0.80434

0.06885

0.73664

0.98842
$-0.01711$

$-0.38937$

$-1.25746$

$-1.76704$

$-1.24263$

$-2.24905$ 
O

C

H

H

C

C

H

C

C

$\mathrm{H}$

C

C

C

$\mathrm{H}$

H

C

$\mathrm{H}$

$\mathrm{H}$

C

$\mathrm{H}$

O

$\mathrm{H}$

O

$\mathrm{H}$

O

C

C

$\mathrm{H}$

H

C

H

$\mathrm{H}$

H

C

O

H

C

$\mathrm{H}$

O

C

H

O

H

$$
\begin{array}{ccc}
2.35635 & 3.53317 & 0.68513 \\
3.74534 & -0.54071 & -0.82699 \\
4.78222 & -0.22477 & -0.65647 \\
3.77645 & -1.21176 & -1.69338 \\
3.30443 & -1.37907 & 0.40869 \\
2.27887 & -2.43258 & 0.00706 \\
2.68846 & -3.20374 & -0.64828 \\
1.04209 & -2.56528 & 0.39765 \\
-0.15941 & -2.82334 & 0.87282 \\
-0.22878 & -3.37327 & 1.81234 \\
-1.51153 & -2.52617 & 0.32954 \\
-1.80747 & 0.48957 & -0.12231 \\
-4.17248 & 0.21163 & 0.32881 \\
-0.88916 & 0.08869 & 0.31747 \\
-4.86423 & -0.27781 & 1.0239 \\
-1.4651 & 1.80246 & -0.87611 \\
-1.2749 & 1.58259 & -1.93526 \\
-2.33099 & 2.47012 & -0.84571 \\
-0.24689 & 2.56874 & -0.36024 \\
-0.20488 & 3.51627 & -0.9268 \\
-0.40751 & 2.85555 & 1.0248 \\
0.36776 & 3.39081 & 1.2802 \\
4.43728 & -2.19783 & 0.76568 \\
5.10124 & -1.61028 & 1.163 \\
-2.48748 & -2.80374 & 1.00819 \\
-2.87375 & 0.65604 & 0.94924 \\
-2.72593 & 1.05806 & 2.20742 \\
-3.57562 & 1.10851 & 2.88381 \\
-1.76327 & 1.37955 & 2.58954 \\
2.87167 & -0.54742 & 1.61808 \\
2.72652 & -1.21209 & 2.47436 \\
3.64113 & 0.1906 & 1.87041 \\
1.93731 & -0.00964 & 1.4377 \\
1.09028 & 1.89466 & -0.59075 \\
-4.78264 & 1.33766 & -0.28173 \\
-5.54458 & 1.00283 & -0.784 \\
-2.45946 & -0.57428 & -1.05473 \\
-2.45797 & -0.22413 & -2.09864 \\
-3.81146 & -0.75964 & -0.63686 \\
-1.75212 & -1.95192 & -1.07441 \\
-2.44226 & -2.6599 & -1.55299 \\
-0.53477 & -1.85758 & -1.81557 \\
-0.76586 & -1.88382 & -2.75674 \\
& & \\
-10 & &
\end{array}
$$




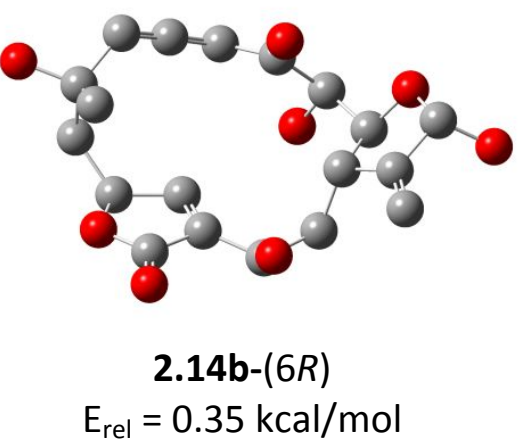

C

C

C

$\mathrm{H}$

C

$\mathrm{H}$

$\mathrm{H}$

C

$\mathrm{H}$

C

C

$\mathrm{H}$

C

$\mathrm{H}$

$\mathrm{H}$

$\mathrm{H}$

O

C

$\mathrm{H}$

C

$\mathrm{H}$

$\mathrm{H}$

C

C

$\mathrm{H}$

$\mathrm{H}$

C

$\mathrm{H}$

C

$\mathrm{H}$

O

C

O

O

$\mathrm{H}$

O

$\mathrm{H}$

C

$\begin{array}{ccc}3.90247 & -2.14547 & 0.4152 \\ 4.66252 & -0.79342 & 0.40606 \\ -3.43254 & -1.04807 & 1.0771 \\ -3.32576 & -1.30916 & 2.10814 \\ -1.18755 & 1.17339 & -1.05783 \\ -0.67143 & 0.74842 & -1.88733 \\ -2.1302 & 1.58911 & -1.38007 \\ -0.26161 & 2.26219 & -0.5452 \\ -0.33072 & 3.09572 & -1.21096 \\ 1.23281 & 1.80287 & -0.57079 \\ 1.90599 & 0.76872 & -1.21074 \\ 1.47245 & -0.09181 & -1.67289 \\ 4.20174 & -0.01152 & 1.6577 \\ 4.38756 & -0.61814 & 2.52482 \\ 4.73749 & 0.90409 & 1.73548 \\ 3.1501 & 0.19138 & 1.58537 \\ 3.55968 & 1.99805 & 0.02245 \\ 3.42307 & 1.13178 & -1.13083 \\ 3.6246 & 1.64047 & -2.0675 \\ 4.56686 & 0.05704 & -0.95966 \\ 5.44938 & 0.64474 & -0.99119 \\ 4.54504 & -0.64669 & -1.77528 \\ 2.78017 & -2.38723 & -0.22498 \\ 1.6471 & -2.6312 & -0.87125 \\ 4.33375 & -2.95607 & 0.96845 \\ 1.67386 & -3.01888 & -1.90125 \\ -1.55999 & 0.08537 & -0.01547 \\ -0.76565 & -0.07499 & 0.68213 \\ -1.92799 & -1.22306 & -0.70253 \\ -2.58639 & -1.00287 & -1.51097 \\ -2.56318 & -1.95435 & 0.33495 \\ 2.27721 & 2.60194 & 0.15842 \\ 2.03779 & 3.64951 & 0.80404 \\ -0.6221 & 2.62849 & 0.78684 \\ 0.03672 & 3.22984 & 1.13439 \\ 6.05922 & -1.11979 & 0.48856 \\ 6.2137 & -1.58841 & 1.3032 \\ 0.31525 & -2.38199 & -0.19843\end{array}$




$\begin{array}{ccc}0.13862 & -2.42693 & 1.06643 \\ -0.72882 & -2.10637 & -1.2519 \\ -1.14781 & -2.98707 & -1.61069 \\ 0.02436 & -1.49686 & -2.29942 \\ 0.67967 & -2.17887 & -2.57841 \\ -2.96449 & 0.36625 & 0.64378 \\ -3.6189 & 1.54258 & 0.80055 \\ -3.17975 & 2.45391 & 0.45195 \\ -4.57802 & 1.56007 & 1.27454 \\ -4.78607 & -1.15457 & 0.6282 \\ -5.33087 & -1.50974 & 1.33434\end{array}$

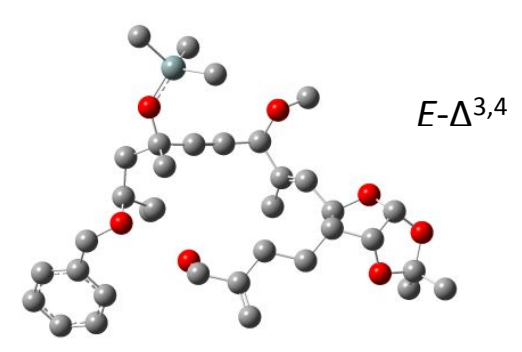

$$
\begin{gathered}
2.19 a-(5 S) \\
E_{\text {rel }}=0.00 \mathrm{kcal} / \mathrm{mol}
\end{gathered}
$$

C

C

C

C

C

$\mathrm{H}$

$\mathrm{H}$

C

$\mathrm{H}$

O

O

C

C

$\mathrm{H}$

$\mathrm{H}$

$\mathrm{H}$

C

$\mathrm{H}$

$\mathrm{H}$

$\mathrm{H}$

C

$\mathrm{H}$

$\mathrm{H}$

C

$\mathrm{H}$

$\begin{array}{lrr}-1.67928 & -1.66752 & -0.07964 \\ -0.65988 & -2.21256 & -0.51393 \\ -3.02508 & -1.1082 & 0.50086 \\ 5.09526 & 1.12474 & -0.09208 \\ 5.9784 & 0.08495 & -0.74705 \\ 5.48575 & 2.12157 & -0.11213 \\ 6.47396 & 0.32809 & -1.66608 \\ 3.80374 & 0.93856 & -0.73273 \\ 3.81415 & 1.22364 & -1.76106 \\ 4.98862 & 0.65513 & 1.24269 \\ 6.9327 & -0.16632 & 0.29602 \\ 6.3744 & 0.59013 & 1.41927 \\ 6.87261 & 2.04791 & 1.33211 \\ 6.44598 & 2.62939 & 2.12705 \\ 7.93337 & 2.06494 & 1.4095 \\ 6.57007 & 2.46933 & 0.38907 \\ 6.749 & -0.05607 & 2.77529 \\ 7.80998 & -0.07198 & 2.87737 \\ 6.32057 & 0.51439 & 3.57697 \\ 6.36743 & -1.05628 & 2.81888 \\ 2.66687 & 1.69009 & -0.06431 \\ 2.53686 & 1.30611 & 0.93594 \\ 2.89187 & 2.7207 & -0.02581 \\ 1.38454 & 1.51767 & -0.87215 \\ 1.53152 & 1.89932 & -1.86479\end{array}$


$\mathrm{H}$

$\mathrm{H}$

$\mathrm{H}$

C

$\mathrm{H}$

$\mathrm{H}$

$\mathrm{H}$

C

$\mathrm{H}$

$\mathrm{H}$

$\mathrm{H}$

C

C

C

C

$\mathrm{H}$

C

$\mathrm{H}$

C

$\mathrm{H}$

$\mathrm{H}$

$\mathrm{H}$

C

$\mathrm{H}$

O

C

$\mathrm{H}$

C

C

$\mathrm{H}$

$\mathrm{H}$

$\mathrm{H}$

O

C

$\mathrm{H}$

$\mathrm{H}$

C

$\mathrm{H}$

C

$\mathrm{H}$

$\mathrm{H}$

C

C
1.11882

$-4.14631$

$-4.24963$

$-5.06335$

$-3.90708$

$-3.35038$

$-3.99127$

$-4.29112$

$-4.7011$

$-3.36192$

$-4.97501$

$-5.6642$

$-6.35254$

$-5.49086$

$-6.07369$

$-2.74864$

$-2.58931$

$-1.81449$

$-3.15594$

$-4.36958$

$-3.3612$

$-5.12445$

$-3.08473$

$-2.80075$

$-4.84947$

$-5.91053$

$-3.82574$

$-2.30796$

$-5.42411$

$-3.61067$

3. 74866

3. 75321

5.00337

2.48283

2.02907

1. 92551

2. 57682

2. 15458

2.39094

3. 62608

$-3.46621$

$-4.66626$

$-5.39855$

$-5.03494$

$-2.45761$

$-2.26521$

$-2.89043$

$-3.82487$

$-2.13768$

0.62935

$-0.86195$
0.49287

$-1.23196$

$-2.25506$

$-0.88283$

$-0.64031$

$-1.90456$

$-3.39057$

$-4.36168$

$-5.32152$

$-4.48244$

$-3.81544$

$-3.16429$

$-2.61877$

$-2.62359$

$-4.12565$

$-4.36073$

$-3.8126$

$-4.48139$

$-5.32225$

3. 32916

4.02714

3.96282

5.35449

3.54425

5.28894

3.4278

5.98594

5.88928

5.76927

6.99772

$-0.56469$

$-0.77717$

$-0.99783$

$-1.21906$

$-0.81993$

$-2.2985$

$-2.90121$

$-2.43839$

$-3.95291$

$-2.71585$

1.22143

1.86837

1.84487

1.35954

1.22015

2. 22989

0.38023

0.74469

0.45988

$-2.90805$

2.90166
$-0.92348$

$-0.55676$

$-0.84534$

$-0.12905$

$-1.42001$

1. 62901

1. 27928

2. 91963

2. 69354

3.43859

3.53624

0.32925

0.94046

$-0.57929$

0.09821

0.14573

$-0.76303$

0.65437

$-0.08677$

$-0.51506$

$-1.18473$

0.47774

$-0.8428$

$-1.95667$

0.82462

0.97484

0.16714

$-1.35209$

1.59004

0.43709

$-0.50176$

0.53623

$-1.09844$

$-1.08641$

$-1.97899$

$-0.45594$

0.8104

1.68688

0.83977

0.7833

$-1.33424$

$-0.87995$

$-1.65247$

$-0.01545$

$-0.312$

$-0.00453$

0.9117

1.29582

1.66195

$-1.05145$

$-1.05349$ 


$\begin{array}{lrrr}\mathrm{H} & -1.74591 & 2.99723 & -0.46935 \\ \mathrm{H} & -0.56884 & 3.86374 & -1.41687 \\ \mathrm{O} & 0.71304 & -2.75297 & -2.45169 \\ \mathrm{C} & 1.96349 & -3.35838 & -2.80622 \\ \mathrm{H} & 1.96533 & -4.38749 & -2.51122 \\ \mathrm{H} & 2.09798 & -3.28666 & -3.86347 \\ \mathrm{H} & 2.76073 & -2.84899 & -2.30441 \\ \mathrm{H} & 0.57614 & -3.94696 & -0.80478 \\ \mathrm{O} & -1.10597 & 2.01945 & -2.15731 \\ \mathrm{H} & -1.80195 & 2.35704 & -2.71871 \\ \mathrm{C} & -1.17285 & 0.60407 & -0.86543 \\ \mathrm{H} & -0.92243 & 0.70013 & -1.90671 \\ \mathrm{C} & -0.36864 & -0.06304 & -0.01361 \\ \mathrm{H} & -0.64973 & -0.15809 & 1.01316 \\ \mathrm{H} & 0.54173 & -0.50082 & -0.37159 \\ \mathrm{C} & 0.27278 & 2.31524 & -0.18052 \\ \mathrm{C} & 0.32001 & 2.49033 & 1.18723 \\ \mathrm{H} & -0.47185 & 3.00273 & 1.70464 \\ \mathrm{H} & 1.15187 & 2.10773 & 1.75771\end{array}$

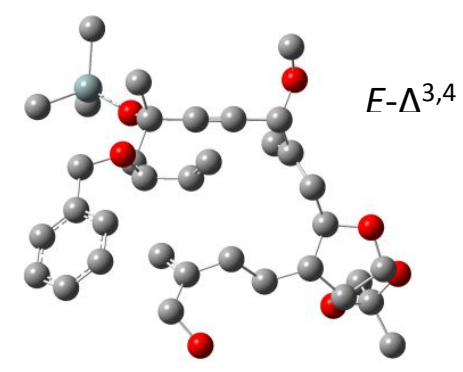

2.19b-(5R)

$\mathrm{E}_{\mathrm{rel}}=6.82 \mathrm{kcal} / \mathrm{mol}$

C

C

C

C

C

$\mathrm{H}$

$\mathrm{H}$

C

$\mathrm{H}$

O

O

C

C

$\mathrm{H}$

$\mathrm{H}$

$\mathrm{H}$

C

$\mathrm{H}$

$$
\begin{array}{rrr}
-1.43492 & -1.79844 & -0.51945 \\
-0.40718 & -2.22165 & -1.00287 \\
-2.78162 & -1.35298 & 0.101 \\
5.25518 & 1.1977 & 0.12587 \\
6.11396 & 0.10948 & -0.5213 \\
5.67598 & 2.185 & 0.11428 \\
6.76402 & 0.48608 & -1.29248 \\
3.9628 & 1.01561 & -0.60023 \\
4.03916 & 1.38852 & -1.61706 \\
5.18379 & 0.83892 & 1.48661 \\
6.82416 & -0.45545 & 0.60491 \\
6.60006 & 0.56964 & 1.57453 \\
7.44472 & 1.80003 & 1.17091 \\
7.25762 & 2.61852 & 1.83739 \\
8.48221 & 1.53304 & 1.21541 \\
7.18948 & 2.09172 & 0.16736 \\
6.96861 & 0.12721 & 3.01109 \\
8.01086 & -0.11469 & 3.05058
\end{array}
$$


$\mathrm{H}$

$\mathrm{H}$

C

$\mathrm{H}$

$\mathrm{H}$

C

$\mathrm{H}$

$\mathrm{H}$

C

$\mathrm{H}$

$\mathrm{H}$

$\mathrm{H}$

O

$\mathrm{Si}$

C

$\mathrm{H}$

$\mathrm{H}$

$\mathrm{H}$

C

$\mathrm{H}$

$\mathrm{H}$

$\mathrm{H}$

C

$\mathrm{H}$

$\mathrm{H}$

$\mathrm{H}$

C

C

C

C

$\mathrm{H}$

C

$\mathrm{H}$

C

$\mathrm{H}$

$\mathrm{H}$

$\mathrm{H}$

C

$\mathrm{H}$

O

C

$\mathrm{H}$

C

C

$\mathrm{H}$

$\mathrm{H}$

$\mathrm{H}$

O

C

$\mathrm{H}$

$\mathrm{H}$

\begin{tabular}{|c|c|c|}
\hline 6.75678 & 0.92943 & 3.69658 \\
\hline 6.3835 & -0.74027 & 3.28444 \\
\hline 2.71565 & 1.65073 & 0.07702 \\
\hline 2.59474 & 1.25367 & 1.07307 \\
\hline 2.82852 & 2.71418 & 0.1366 \\
\hline 1.48564 & 1.30137 & -0.78396 \\
\hline 1.64914 & 1.66019 & -1.78967 \\
\hline 1.35547 & 0.23117 & -0.79942 \\
\hline-3.89227 & -1.40145 & -0.96264 \\
\hline-3.96982 & -2.39266 & -1.36783 \\
\hline-4.8259 & -1.13059 & -0.501 \\
\hline-3.66503 & -0.71081 & -1.752 \\
\hline-3.08076 & -2.27346 & 1.15736 \\
\hline-4.59605 & -2.92193 & 1.383 \\
\hline-4.50452 & -4.15953 & 2.857 \\
\hline-5.46804 & -4.60588 & 3.007 \\
\hline-3.78493 & -4.92113 & 2.635 \\
\hline-4.21158 & -3.62966 & 3.74 \\
\hline-5.91365 & -1.54555 & 1.78 \\
\hline-5.62765 & -1.02974 & 2.683 \\
\hline-5.97589 & -0.84666 & 0.977 \\
\hline-6.87375 & -2.00968 & 1.93399 \\
\hline-5.12491 & -3.85089 & -0.231 \\
\hline-5.16371 & -3.15572 & -1.04867 \\
\hline-4.41006 & -4.61895 & -0.444 \\
\hline-6.09518 & -4.28962 & -0.0876 \\
\hline-4.12118 & 3.32131 & -0.548 \\
\hline-3.09903 & 4.02611 & -1.20868 \\
\hline-4.86222 & 3.97345 & 0.46507 \\
\hline-2.81256 & 5.3523 & -0.86369 \\
\hline-2.53828 & 3.54449 & -1.9792 \\
\hline-4.56525 & 5.30761 & 0.807 \\
\hline-5.65488 & 3.45486 & 0.97235 \\
\hline-3.53011 & 5.98986 & 0.150 \\
\hline-2.03579 & 5.87909 & -1.37392 \\
\hline-5.1214 & 5.80262 & 1.573 \\
\hline-3.28952 & 6.99612 & 0.42167 \\
\hline 3.92482 & -0.50016 & -0.5576 \\
\hline 3.89101 & -0.83125 & 0.45425 \\
\hline 5.18917 & -0.85416 & -1.15044 \\
\hline 2.71046 & -1.08903 & -1.294 \\
\hline 2.32299 & -0.60069 & -2.16613 \\
\hline 2.12105 & -2.21847 & -0.83852 \\
\hline 2.66082 & -2.92115 & 0.4219 \\
\hline 2.14737 & -2.54539 & 1.290 \\
\hline 2.49768 & -3.98301 & 0.33882 \\
\hline 3.71413 & -2.72755 & 0.51681 \\
\hline-3.21063 & 1.22502 & -1.4326 \\
\hline-4.40474 & 1.84371 & -0.955 \\
\hline-5.139 & 1.8216 & -1.742 \\
\hline-4 & 1.302 & -0.1 \\
\hline
\end{tabular}




$\begin{array}{ccr}-2.25524 & 1.13367 & -0.38217 \\ -2.16425 & 2.08125 & 0.09298 \\ -0.25404 & -4.73527 & -2.16387 \\ -0.2139 & -4.46616 & -3.20568 \\ -0.24919 & -5.80757 & -2.06693 \\ -1.15063 & -4.33429 & -1.72037 \\ -2.69106 & 0.0831 & 0.68549 \\ -3.64851 & 0.35413 & 1.07654 \\ -1.96157 & 0.08551 & 1.47993 \\ 0.894 & -4.20839 & -1.49665 \\ 0.9038 & -2.77705 & -1.60007 \\ 0.96186 & -2.47963 & -2.62871 \\ -0.05954 & 3.4437 & -0.51671 \\ -1.12205 & 3.62189 & -0.55235 \\ 0.37043 & 4.05154 & 0.25646 \\ 0.5371 & 3.76641 & -1.78107 \\ 0.3802 & 4.68379 & -1.98505 \\ -0.90372 & 0.73262 & -0.97464 \\ -0.00396 & 1.12806 & -0.55248 \\ -0.85516 & -0.11624 & -2.03433 \\ -1.76207 & -0.51418 & -2.45517 \\ 0.09097 & -0.38521 & -2.44986 \\ 0.2167 & 1.97116 & -0.20964 \\ -0.64746 & 1.26887 & 0.56275 \\ -1.67728 & 1.18932 & 0.28337 \\ -0.29874 & 0.79228 & 1.45503\end{array}$

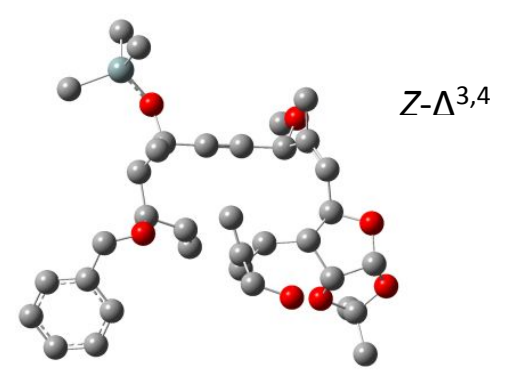

2.19c- $(5 S)$

$E_{\text {rel }}=7.21 \mathrm{kcal} / \mathrm{mol}$

$\mathrm{C}$
$\mathrm{C}$
$\mathrm{C}$
$\mathrm{H}$
$\mathrm{H}$
$\mathrm{C}$
$\mathrm{H}$
$\mathrm{O}$
$\mathrm{O}$
$\mathrm{C}$
$\mathrm{C}$
$\mathrm{H}$

\begin{tabular}{lrr}
2.752 & -1.26455 & \multicolumn{1}{c}{0.0088} \\
-4.45323 & 1.00443 & -0.34172 \\
-5.31927 & 0.68635 & 0.88269 \\
-4.40786 & 2.07675 & -0.57167 \\
-5.45501 & 1.49537 & 1.60649 \\
-3.08231 & 0.3898 & -0.02904 \\
-2.50878 & 1.13394 & 0.54018 \\
-5.1163 & 0.30216 & -1.39207 \\
-6.58811 & 0.39437 & 0.36209 \\
-6.45863 & -0.02419 & -1.00211 \\
-7.45225 & 0.77568 & -1.83955 \\
-7.36851 & 0.49882 & -2.89511
\end{tabular}


H

$\mathrm{H}$

C

$\mathrm{H}$

$\mathrm{H}$

$\mathrm{H}$

C

$\mathrm{H}$

$\mathrm{H}$

$\mathrm{H}$

O

$\mathrm{Si}$

C

$\mathrm{H}$

$\mathrm{H}$

$\mathrm{H}$

C

$\mathrm{H}$

$\mathrm{H}$

$\mathrm{H}$

C

$\mathrm{H}$

$\mathrm{H}$

$\mathrm{H}$

C

$\mathrm{H}$

O

C

$\mathrm{H}$

$\mathrm{H}$

C

C

$\mathrm{H}$

C

$\mathrm{H}$

$\mathrm{H}$

$\mathrm{H}$

C

$\mathrm{H}$

$\mathrm{H}$

C

C

C

$\mathrm{H}$

C

$\mathrm{H}$

C

$\mathrm{H}$

O

C

$\mathrm{H}$

\begin{tabular}{|c|c|c|}
\hline-8.47568 & 0.58063 & -1.50403 \\
\hline-7.24834 & 1.84556 & -1.73714 \\
\hline-6.6543 & -1.53355 & -1.11938 \\
\hline-7.67576 & -1.80445 & -0.8344 \\
\hline-6.47769 & -1.86116 & -2.14907 \\
\hline-5.95964 & -2.05096 & -0.45309 \\
\hline 3.44365 & -1.1412 & 1.38096 \\
\hline 3.48539 & -2.11848 & 1.86924 \\
\hline 4.46407 & -0.7592 & 1.26102 \\
\hline 2.88515 & -0.4475 & 2.0119 \\
\hline 3.42256 & -2.21482 & -0.83336 \\
\hline 5.00729 & -2.71264 & -1.05541 \\
\hline 4.9101 & -3.81725 & -2.576 \\
\hline 5.89106 & -4.24052 & -2.82552 \\
\hline 4.21751 & -4.65037 & -2.41 \\
\hline 4.5537 & -3.25851 & -3.4 \\
\hline 6.17 & -1.26519 & -1.3 \\
\hline 5.84001 & -0.66648 & -2.24615 \\
\hline 6.28548 & -0.59323 & -0.53265 \\
\hline 7.18172 & -1.64274 & -1.63 \\
\hline 5.65 & -3.712 & 0.41 \\
\hline 5.811 & -3.09822 & 1.3 \\
\hline 4.96192 & -4.51821 & 0.67738 \\
\hline 6.61834 & -4.17472 & 0.15817 \\
\hline-3.45297 & -0.78156 & 0.92624 \\
\hline-3.60937 & -1.69389 & 0.3 \\
\hline-4.70868 & -0.41095 & 1.53883 \\
\hline 2.76596 & 0.08841 & -0.7661 \\
\hline 3.81173 & 0.42217 & -0.76115 \\
\hline 2.5192 & -0.14149 & -1.80 \\
\hline-1.4612 & -1.87346 & 2.17 \\
\hline-2.48897 & -1.02048 & 05989 \\
\hline-2.70907 & -0.38589 & 2.91788 \\
\hline-0.65779 & -1.97394 & 3.44 \\
\hline 0.40215 & -1.76156 & 3.2 \\
\hline-1.02679 & -1.27343 & 4.19 \\
\hline-0.71451 & -2.98969 & 3.84991 \\
\hline-1.66544 & 1.07769 & -2.09678 \\
\hline-2.31302 & 1.32771 & -2.9 \\
\hline-0.74512 & 0.69163 & -2.56077 \\
\hline 1.39594 & -1.80642 & 0.24286 \\
\hline 0.32513 & -2.27732 & 0.5578 \\
\hline-0.98529 & -2.77301 & 1.03675 \\
\hline-2.99021 & -0.61304 & -1.9063 \\
\hline-2.29521 & -0.05753 & -1.26938 \\
\hline-1.51101 & -0.76326 & -0.97709 \\
\hline 1.85947 & 1.27892 & -0.34354 \\
\hline 2.23375 & 2.1455 & -0.90177 \\
\hline 1.94672 & 1.57379 & 1.06316 \\
\hline 3.01406 & 2.43707 & 1.42549 \\
\hline 3.08249 & 2.35374 & 2.51752 \\
\hline
\end{tabular}




$\begin{array}{lccc}\mathrm{H} & 3.97379 & 2.08118 & 1.01916 \\ \mathrm{C} & 2.79798 & 3.8895 & 1.0326 \\ \mathrm{C} & 3.89193 & 4.7161 & 0.75254 \\ \mathrm{C} & 1.50886 & 4.43518 & 0.99591 \\ \mathrm{C} & 3.70642 & 6.06687 & 0.45292 \\ \mathrm{H} & 4.89792 & 4.30064 & 0.76912 \\ \mathrm{C} & 1.32071 & 5.78253 & 0.68695 \\ \mathrm{H} & 0.65921 & 3.78948 & 1.19675 \\ \mathrm{C} & 2.41844 & 6.60355 & 0.41836 \\ \mathrm{H} & 4.56611 & 6.69618 & 0.23748 \\ \mathrm{H} & 0.31472 & 6.19348 & 0.65728 \\ \mathrm{H} & 2.27039 & 7.65333 & 0.17926 \\ \mathrm{C} & -0.46686 & 3.29374 & -2.24673 \\ \mathrm{H} & -0.34991 & 4.29473 & -1.82503 \\ \mathrm{H} & 0.53761 & 2.88743 & -2.43206 \\ \mathrm{H} & -1.70485 & -2.75113 & 0.20324 \\ \mathrm{O} & -0.90487 & -4.10444 & 1.54185 \\ \mathrm{C} & -0.57644 & -5.0652 & 0.55368 \\ \mathrm{H} & -1.32322 & -5.08107 & -0.25635 \\ \mathrm{H} & 0.4133 & -4.87681 & 0.11526 \\ \mathrm{H} & -0.57267 & -6.0372 & 1.05313 \\ \mathrm{O} & -1.08649 & 3.43595 & -3.52764 \\ \mathrm{H} & -0.44636 & 3.77548 & -4.15736 \\ \mathrm{C} & 0.37487 & 1.05001 & -0.68292 \\ \mathrm{H} & -0.37693 & 1.65646 & -0.22258 \\ \mathrm{C} & 0.01735 & 0.08312 & -1.56261 \\ \mathrm{H} & -0.79953 & -0.57147 & -1.34098 \\ \mathrm{H} & 0.5538 & -0.02609 & -2.48196 \\ \mathrm{C} & -1.27707 & 2.35982 & -1.37499 \\ \mathrm{C} & -1.62016 & 2.64061 & -0.09436 \\ \mathrm{H} & -2.60816 & 2.42853 & 0.25748 \\ \mathrm{H} & -0.9006 & 3.0759 & 0.56719\end{array}$

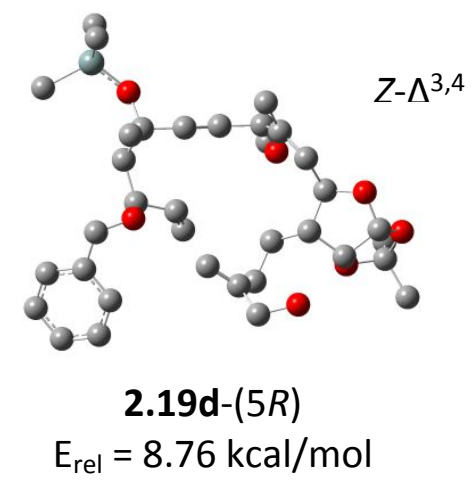




\begin{tabular}{|c|c|c|c|}
\hline $\mathrm{C}$ & 2.87773 & -1.2829 & 0.15199 \\
\hline C & -4.4018 & 0.90006 & -0.26875 \\
\hline $\mathrm{C}$ & -5.24616 & 0.63042 & 0.98222 \\
\hline $\mathrm{H}$ & -4.33787 & 1.96578 & -0.52406 \\
\hline $\mathrm{H}$ & -5.35658 & 1.46411 & 1.68236 \\
\hline C & -3.03697 & 0.26383 & 0.02861 \\
\hline $\mathrm{H}$ & -2.44219 & 1.01077 & 0.57284 \\
\hline O & -5.10506 & 0.19438 & -1.29099 \\
\hline O & -6.53231 & 0.34562 & 0.49498 \\
\hline C & -6.43625 & -0.1183 & -0.85589 \\
\hline C & -7.45001 & 0.65433 & -1.69522 \\
\hline $\mathrm{H}$ & -7.39205 & 0.34258 & -2.74279 \\
\hline $\mathrm{H}$ & -8.46515 & 0.47154 & -1.32875 \\
\hline $\mathrm{H}$ & -7.24291 & 1.72665 & -1.63316 \\
\hline C & -6.63703 & -1.6309 & -0.91969 \\
\hline $\mathrm{H}$ & -7.64997 & -1.89044 & -0.59588 \\
\hline $\mathrm{H}$ & -6.49178 & -1.99039 & -1.9437 \\
\hline $\mathrm{H}$ & -5.92263 & -2.12891 & -0.25977 \\
\hline C & 3.57465 & -1.04091 & 1.50631 \\
\hline $\mathrm{H}$ & 3.66461 & -1.98163 & 2.05625 \\
\hline $\mathrm{H}$ & 4.57588 & -0.62347 & 1.34919 \\
\hline $\mathrm{H}$ & 2.99192 & -0.33188 & 2.09675 \\
\hline 0 & 3.58023 & -2.2563 & -0.63517 \\
\hline $\mathrm{Si}$ & 5.1753 & -2.73386 & -0.82182 \\
\hline $\mathrm{C}$ & 5.11391 & -3.90096 & -2.29746 \\
\hline $\mathrm{H}$ & 6.10588 & -4.31201 & -2.52229 \\
\hline $\mathrm{H}$ & 4.43898 & -4.74209 & -2.10231 \\
\hline $\mathrm{H}$ & 4.75163 & -3.38699 & -3.19503 \\
\hline C & 6.32774 & -1.28182 & -1.20212 \\
\hline $\mathrm{H}$ & 5.98658 & -0.71901 & -2.07886 \\
\hline $\mathrm{H}$ & 6.41566 & -0.57865 & -0.36528 \\
\hline $\mathrm{H}$ & 7.33767 & -1.65215 & -1.42087 \\
\hline C & 5.82093 & -3.66581 & 0.69103 \\
\hline $\mathrm{H}$ & 5.95076 & -3.01737 & 1.56442 \\
\hline $\mathrm{H}$ & 5.13796 & -4.47534 & 0.97427 \\
\hline $\mathrm{H}$ & 6.796 & -4.11867 & 0.46938 \\
\hline $\mathrm{C}$ & -3.41033 & -0.87979 & 1.01699 \\
\hline $\mathrm{H}$ & -3.59428 & -1.80523 & 0.46695 \\
\hline O & -4.64088 & -0.45387 & 1.65765 \\
\hline C & 2.82789 & 0.01403 & -0.71051 \\
\hline $\mathrm{H}$ & 3.86052 & 0.38483 & -0.74331 \\
\hline $\mathrm{H}$ & 2.5779 & -0.29441 & -1.73093 \\
\hline C & -1.36481 & -1.89535 & 2.29623 \\
\hline $\mathrm{C}$ & -2.42387 & -1.08868 & 2.13908 \\
\hline $\mathrm{H}$ & -2.65359 & -0.43515 & 2.98087 \\
\hline $\mathrm{C}$ & -0.57361 & -1.86496 & 3.58602 \\
\hline $\mathrm{H}$ & 0.47696 & -1.60543 & 3.40793 \\
\hline $\mathrm{H}$ & -0.99063 & -1.13692 & 4.28689 \\
\hline $\mathrm{H}$ & -0.58098 & -2.84901 & 4.0758 \\
\hline C & -1.65443 & 0.9011 & -2.07448 \\
\hline $\mathrm{H}$ & -2.3594 & 1.21519 & -2.86043 \\
\hline
\end{tabular}




\begin{tabular}{|c|c|c|c|}
\hline $\mathrm{H}$ & -0.80768 & 0.47109 & -2.63181 \\
\hline C & 1.55012 & -1.8635 & 0.4417 \\
\hline $\mathrm{C}$ & 0.50427 & -2.36273 & 0.79312 \\
\hline $\mathrm{C}$ & -0.7938 & -2.88195 & 1.28101 \\
\hline $\mathrm{H}$ & -0.59872 & -3.83239 & 1.81299 \\
\hline $\mathrm{H}$ & -2.99381 & -0.7683 & -1.83357 \\
\hline $\mathrm{C}$ & -2.27847 & -0.21412 & -1.21832 \\
\hline $\mathrm{H}$ & -1.51193 & -0.93587 & -0.92645 \\
\hline O & -1.71314 & -3.14055 & 0.23177 \\
\hline C & -1.29757 & -4.17711 & -0.6433 \\
\hline $\mathrm{H}$ & -0.35588 & -3.92597 & -1.14887 \\
\hline $\mathrm{H}$ & -1.16821 & -5.12669 & -0.10108 \\
\hline $\mathrm{H}$ & -2.09026 & -4.29332 & -1.38583 \\
\hline $\mathrm{C}$ & 1.88201 & 1.19566 & -0.35378 \\
\hline $\mathrm{H}$ & 2.23242 & 2.04165 & -0.95619 \\
\hline 0 & 1.96161 & 1.56827 & 1.03658 \\
\hline $\mathrm{C}$ & 2.9873 & 2.50128 & 1.34212 \\
\hline $\mathrm{H}$ & 3.06512 & 2.48367 & 2.43665 \\
\hline $\mathrm{H}$ & 3.96153 & 2.17017 & 0.94912 \\
\hline $\mathrm{C}$ & 2.69969 & 3.91712 & 0.8698 \\
\hline $\mathrm{C}$ & 3.75043 & 4.77366 & 0.52224 \\
\hline $\mathrm{C}$ & 1.38662 & 4.40181 & 0.82483 \\
\hline $\mathrm{C}$ & 3.49919 & 6.09493 & 0.14811 \\
\hline $\mathrm{H}$ & 4.77439 & 4.40479 & 0.54417 \\
\hline C & 1.13281 & 5.71905 & 0.44206 \\
\hline $\mathrm{H}$ & 0.57026 & 3.7315 & 1.0764 \\
\hline C & 2.18783 & 6.57095 & 0.10642 \\
\hline $\mathrm{H}$ & 4.32595 & 6.74794 & -0.11944 \\
\hline $\mathrm{H}$ & 0.10884 & 6.08236 & 0.40674 \\
\hline $\mathrm{H}$ & 1.98847 & 7.59725 & -0.19043 \\
\hline C & -0.38692 & 3.06795 & -2.31905 \\
\hline $\mathrm{H}$ & -0.17171 & 4.05083 & -1.89376 \\
\hline $\mathrm{H}$ & 0.56587 & 2.62557 & -2.64312 \\
\hline 0 & -1.15937 & 3.27301 & -3.50487 \\
\hline $\mathrm{H}$ & -0.58698 & 3.58268 & -4.21062 \\
\hline $\mathrm{C}$ & 0.40521 & 0.91099 & -0.68487 \\
\hline $\mathrm{H}$ & -0.36048 & 1.19467 & 0.00661 \\
\hline $\mathrm{C}$ & 0.07208 & 0.30242 & -1.84902 \\
\hline $\mathrm{H}$ & -0.64365 & -0.49294 & -1.85549 \\
\hline H & 0.52633 & 0.61837 & -2.76484 \\
\hline $\mathrm{C}$ & -1.1189 & 2.14092 & -1.37297 \\
\hline$C$ & -1.28032 & 2.39324 & -0.05129 \\
\hline & -2.09195 & 1.94959 & 0.48661 \\
\hline & -0.59378 & 3.03832 & 0.4561 \\
\hline
\end{tabular}




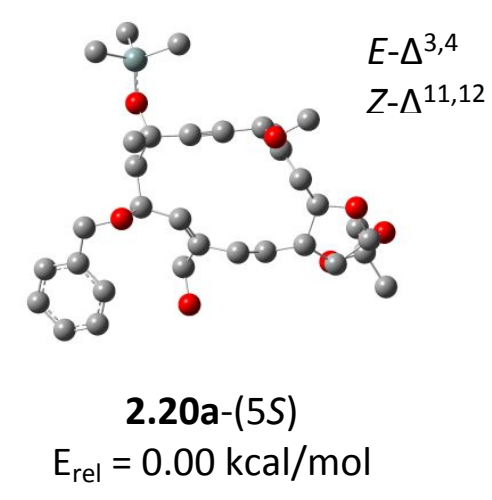

C

C

C

C

C

$\mathrm{H}$

$\mathrm{H}$

C

$\mathrm{H}$

O

O

C

C

$\mathrm{H}$

$\mathrm{H}$

$\mathrm{H}$

C

$\mathrm{H}$

$\mathrm{H}$

$\mathrm{H}$

C

$\mathrm{H}$

$\mathrm{H}$

C

$\mathrm{H}$

$\mathrm{H}$

C

C

$\mathrm{H}$

C

$\mathrm{H}$

$\mathrm{H}$

$\mathrm{H}$

O

$\mathrm{Si}$

C

$\mathrm{H}$

$\mathrm{H}$

$\mathrm{H}$

$$
\begin{array}{lcr}
-1.59176 & -1.92177 & -0.33541 \\
-0.54286 & -2.41911 & -0.67972 \\
-2.92408 & -1.40959 & 0.05543 \\
5.11597 & 1.31818 & -0.46403 \\
5.98654 & 0.0678 & -0.76449 \\
5.40359 & 2.20378 & -1.04583 \\
6.63631 & 0.1296 & -1.64206 \\
3.69058 & 0.83756 & -0.74778 \\
3.55368 & 0.90843 & -1.83653 \\
5.30619 & 1.54633 & 0.92911 \\
6.78615 & -0.10254 & 0.3926 \\
6.55555 & 0.97048 & 1.31154 \\
7.68227 & 2.00165 & 1.19296 \\
7.51272 & 2.83436 & 1.88313 \\
8.6462 & 1.53875 & 1.42629 \\
7.73145 & 2.39948 & 0.17402 \\
6.41736 & 0.40465 & 2.71733 \\
7.34994 & -0.07922 & 3.02249 \\
6.18692 & 1.20487 & 3.42733 \\
5.61226 & -0.33447 & 2.73947 \\
2.53477 & 1.56101 & -0.03082 \\
2.2253 & 0.96498 & 0.83689 \\
2.87897 & 2.51969 & 0.37198 \\
1.32157 & 1.77235 & -0.96731 \\
1.54351 & 2.62595 & -1.62814 \\
1.21674 & 0.89673 & -1.61668 \\
0.00231 & 2.00769 & -0.25853 \\
-1.04031 & 1.2128 & -0.54999 \\
-0.8924 & 0.43738 & -1.29738 \\
-3.84658 & -1.39137 & -1.17694 \\
-3.90029 & -2.38891 & -1.62395 \\
-4.8525 & -1.08337 & -0.87407 \\
-3.48251 & -0.68755 & -1.92662 \\
-3.4601 & -2.2687 & 1.07797 \\
-4.03194 & -3.84929 & 1.09224 \\
-4.0454 & -4.29797 & 2.92119 \\
-4.45146 & -5.30476 & 3.07962 \\
-3.03349 & -4.27343 & 3.34138 \\
-4.66028 & -3.59531 & 3.49516
\end{array}
$$




\begin{tabular}{|c|c|c|c|}
\hline $\mathrm{C}$ & -5.79339 & -3.94389 & 0.41045 \\
\hline $\mathrm{H}$ & -6.4511 & -3.22269 & 0.90996 \\
\hline $\mathrm{H}$ & -5.83648 & -3.74422 & -0.66592 \\
\hline $\mathrm{H}$ & -6.21327 & -4.94428 & 0.57738 \\
\hline $\mathrm{C}$ & -2.92825 & -5.04876 & 0.13844 \\
\hline $\mathrm{H}$ & -2.86028 & -4.79882 & -0.92579 \\
\hline $\mathrm{H}$ & -1.90909 & -5.05846 & 0.53998 \\
\hline $\mathrm{H}$ & -3.33083 & -6.06714 & 0.21692 \\
\hline $\mathrm{C}$ & -4.25769 & 3.7471 & -0.36689 \\
\hline C & -3.29766 & 4.4752 & -1.08134 \\
\hline C & -5.02412 & 4.40564 & 0.60086 \\
\hline C & -3.11343 & 5.83552 & -0.83416 \\
\hline $\mathrm{H}$ & -2.68983 & 3.95972 & -1.81901 \\
\hline $\mathrm{C}$ & -4.84683 & 5.76919 & 0.84453 \\
\hline $\mathrm{H}$ & -5.76631 & 3.84833 & 1.16953 \\
\hline $\mathrm{C}$ & -3.88985 & 6.488 & 0.12692 \\
\hline $\mathrm{H}$ & -2.36318 & 6.38864 & -1.39368 \\
\hline $\mathrm{H}$ & -5.44949 & 6.26618 & 1.60044 \\
\hline $\mathrm{H}$ & -3.74603 & 7.54815 & 0.31826 \\
\hline $\mathrm{C}$ & 3.82628 & -0.66495 & -0.40417 \\
\hline $\mathrm{H}$ & 3.88673 & -0.76145 & 0.68638 \\
\hline O & 5.10622 & -1.01048 & -0.97904 \\
\hline C & 2.74906 & -1.53158 & -0.96897 \\
\hline $\mathrm{H}$ & 2.63216 & -1.48464 & -2.04987 \\
\hline $\mathrm{C}$ & 1.88883 & -2.29113 & -0.27752 \\
\hline $\mathrm{C}$ & 1.87534 & -2.50534 & 1.21474 \\
\hline $\mathrm{H}$ & 0.95284 & -2.10837 & 1.65579 \\
\hline $\mathrm{H}$ & 1.89907 & -3.57907 & 1.44667 \\
\hline $\mathrm{H}$ & 2.72289 & -2.03793 & 1.71919 \\
\hline 0 & -3.32684 & 1.5722 & -1.05581 \\
\hline C & -4.49824 & 2.27888 & -0.67767 \\
\hline $\mathrm{H}$ & -5.17121 & 2.18633 & -1.5395 \\
\hline $\mathrm{H}$ & -5.00367 & 1.79734 & 0.17348 \\
\hline $\mathrm{C}$ & -2.42619 & 1.25679 & 0.0308 \\
\hline $\mathrm{H}$ & -2.48996 & 2.06192 & 0.77307 \\
\hline $\mathrm{C}$ & -2.85769 & -0.02948 & 0.77412 \\
\hline $\mathrm{H}$ & -3.86564 & 0.12605 & 1.17784 \\
\hline $\mathrm{H}$ & -2.19851 & -0.15195 & 1.641 \\
\hline $\mathrm{C}$ & 0.75628 & -3.02667 & -1.01306 \\
\hline $\mathrm{C}$ & -0.01353 & 3.15248 & 0.72601 \\
\hline $\mathrm{H}$ & -1.01154 & 3.38822 & 1.10104 \\
\hline $\mathrm{H}$ & 0.63059 & 2.94357 & 1.59038 \\
\hline 0 & 0.90187 & -3.04708 & -2.42696 \\
\hline $\mathrm{C}$ & 1.75354 & -4.08569 & -2.88845 \\
\hline $\mathrm{H}$ & 1.35261 & -5.07604 & -2.62292 \\
\hline $\mathrm{H}$ & 1.78794 & -3.99689 & -3.97701 \\
\hline $\mathrm{H}$ & 2.77164 & -3.99438 & -2.48738 \\
\hline $\mathrm{H}$ & 0.73888 & -4.06806 & -0.6403 \\
\hline 0 & 0.50537 & 4.3354 & 0.11255 \\
\hline $\mathrm{H}$ & 0.60541 & 5.02263 & 0.77535 \\
\hline
\end{tabular}




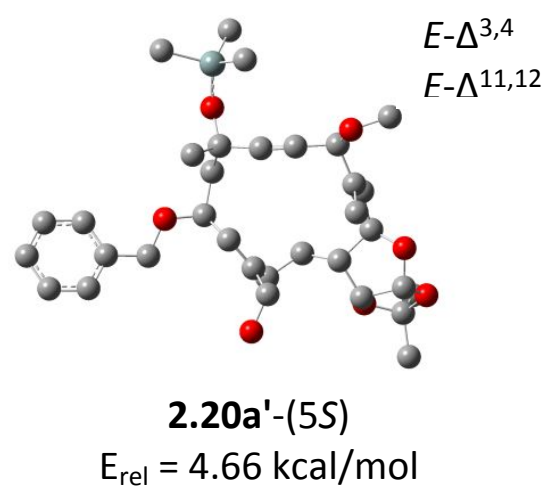

C

C

C

C

C

$\mathrm{H}$

$\mathrm{H}$

C

C

$\mathrm{H}$

C

C

$\mathrm{H}$

$\mathrm{H}$

C

$\mathrm{H}$

O

o

C

C

$\mathrm{H}$

$\mathrm{H}$

$\mathrm{H}$

C

$\mathrm{H}$

$\mathrm{H}$

$\mathrm{H}$

C

$\mathrm{H}$

$\mathrm{H}$

C

$\mathrm{H}$

$\mathrm{H}$

C

C

C

$\mathrm{H}$

C
1.06099

0.03539

2. 30586

$-1.26991$

1.94089

2.77915

1.06914

$-2.36525$

$-2.98999$

$-2.74766$

$-4.44972$

$-5.56506$

$-4.29364$

$-5.95239$

$-3.22222$

$-2.77577$

$-4.90011$

$-6.61176$

$-6.31632$

$-7.0367$

$-6.80458$

$-8.11956$

$-6.72525$

$-6.68932$

$-7.76554$

$-6.42239$

$-6.15571$

$-2.14349$

$-1.50302$

$-2.62316$

$-1.23819$

$-1.86206$

$-0.59159$

$-0.39365$

1.69642

0.8147

1. 30892

3.29024
2.02562

2. 62651

1. 39835

3. 29653

0.17223

0.05568

0.45824

2.23919

1. 78154

2.25757

$-1.65622$

$-0.64747$

$-2.44308$

$-0.73062$

$-0.76209$

$-0.5828$

$-2.21707$

$-0.88793$

$-2.04826$

$-3.26745$

$-4.16431$

$-3.109$

$-3.4375$

$-1.77857$

$-1.6018$

$-2.63568$

$-0.89465$

$-1.28601$

$-0.44367$

$-1.66035$

$-2.40032$

$-3.25693$

$-2.75605$

$-1.96718$

$-1.2292$

$-1.38527$

$-1.09925$

1.0668
$-0.32181$

$-0.55375$

0.17515

$-0.64353$

1.07041

1.76452

1.66795

$-0.83551$

0.25975

1.20967

$-0.23834$

$-0.63236$

$-0.98769$

$-1.65205$

$-0.0197$

$-1.00082$

0.99038

0.2903

1. 07232

0.48778

1.07101

0.49759

$-0.54821$

2.52227

2. 60814

3.14807

2.88116

0.93319

1. 22241

1. 84445

0.34657

0.05762

1. 15812

$-0.84189$

0.4764

$-0.7416$

$-1.66894$

$-0.95802$ 


\begin{tabular}{|c|c|c|c|}
\hline $\mathrm{H}$ & 3.56601 & 1.98101 & -1.49237 \\
\hline $\mathrm{H}$ & 4.18843 & 0.60592 & -0.54068 \\
\hline $\mathrm{H}$ & 2.85614 & 0.37346 & -1.6809 \\
\hline 0 & 2.91788 & 2.30764 & 1.10512 \\
\hline C & -2.54141 & 1.74031 & -2.24521 \\
\hline $\mathrm{H}$ & -3.4575 & 1.15957 & -2.35347 \\
\hline $\mathrm{H}$ & -2.57359 & 2.58453 & -2.94152 \\
\hline $\mathrm{H}$ & -1.68081 & 1.127 & -2.5451 \\
\hline Si & 3.61913 & 3.83001 & 0.94651 \\
\hline $\mathrm{C}$ & 3.20811 & 4.7089 & 2.56164 \\
\hline $\mathrm{H}$ & 3.69868 & 5.68857 & 2.6189 \\
\hline $\mathrm{H}$ & 2.12801 & 4.86743 & 2.66024 \\
\hline $\mathrm{H}$ & 3.53737 & 4.11907 & 3.42488 \\
\hline C & 5.49166 & 3.62959 & 0.79167 \\
\hline $\mathrm{H}$ & 5.89786 & 3.04964 & 1.62875 \\
\hline $\mathrm{H}$ & 5.77001 & 3.11355 & -0.13465 \\
\hline $\mathrm{H}$ & 5.99243 & 4.6063 & 0.78949 \\
\hline C & 2.97736 & 4.83214 & -0.5202 \\
\hline $\mathrm{H}$ & 3.25639 & 4.3936 & -1.48512 \\
\hline $\mathrm{H}$ & 1.8879 & 4.93296 & -0.51276 \\
\hline $\mathrm{H}$ & 3.41412 & 5.8391 & -0.48354 \\
\hline C & -2.30055 & 5.1996 & -1.59449 \\
\hline $\mathrm{H}$ & -2.2789 & 5.75535 & -0.64383 \\
\hline $\mathrm{H}$ & -2.17258 & 5.90194 & -2.42158 \\
\hline $\mathrm{H}$ & -3.27954 & 4.70747 & -1.68576 \\
\hline 0 & -5.02748 & 0.64655 & -0.50491 \\
\hline C & -3.88718 & 0.57714 & 0.381 \\
\hline $\mathrm{H}$ & -4.25097 & 0.50987 & 1.4162 \\
\hline $\mathrm{H}$ & 1.27648 & -1.82362 & 1.30431 \\
\hline 0 & 2.99895 & -1.75862 & 0.16888 \\
\hline $\mathrm{C}$ & 3.04187 & -3.16865 & 0.0111 \\
\hline $\mathrm{H}$ & 2.57694 & -3.64695 & 0.89294 \\
\hline $\mathrm{H}$ & 2.459 & -3.48985 & -0.86458 \\
\hline C & 4.47768 & -3.62176 & -0.12638 \\
\hline C & 4.79608 & -4.70522 & -0.95235 \\
\hline C & 5.5014 & -2.99953 & 0.59864 \\
\hline C & 6.10953 & -5.16847 & -1.04658 \\
\hline $\mathrm{H}$ & 4.01066 & -5.18929 & -1.52934 \\
\hline C & 6.81592 & -3.4549 & 0.49868 \\
\hline $\mathrm{H}$ & 5.26052 & -2.14949 & 1.22897 \\
\hline C & 7.12429 & -4.54291 & -0.32119 \\
\hline $\mathrm{H}$ & 6.34019 & -6.01074 & -1.69382 \\
\hline $\mathrm{H}$ & 7.60201 & -2.95912 & 1.06263 \\
\hline $\mathrm{H}$ & 8.14897 & -4.8971 & -0.39751 \\
\hline C & -0.95995 & -2.2669 & -2.21425 \\
\hline $\mathrm{H}$ & -0.31336 & -1.88733 & -3.01156 \\
\hline $\mathrm{H}$ & -1.96267 & -1.85104 & -2.3678 \\
\hline 0 & -1.22694 & 4.2763 & -1.67666 \\
\hline $\mathrm{H}$ & -1.44527 & 3.80274 & 0.32183 \\
\hline O & -1.08575 & -3.68057 & -2.38918 \\
\hline $\mathrm{H}$ & -1.17169 & -3.8814 & -3.324 \\
\hline
\end{tabular}




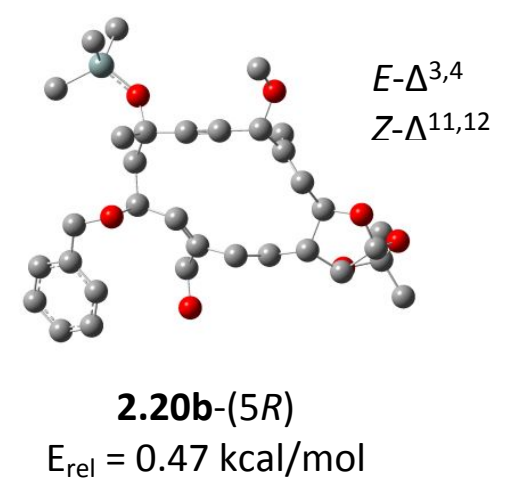

$\begin{array}{llll}\text { C } & 0 . & 0 . & 0 . \\ \mathrm{C} & 1.21119 & 0 . & 0 . \\ \mathrm{C} & -1.47757 & 0.08883 & 0 . \\ \mathrm{C} & 4.59059 & -5.86098 & -0.56356 \\ \mathrm{C} & 5.93091 & -5.11056 & -0.79714 \\ \mathrm{H} & 4.67475 & -6.70326 & 0.1357 \\ \mathrm{H} & 6.75125 & -5.36192 & -0.11889 \\ \mathrm{C} & 3.64424 & -4.75849 & -0.07985 \\ \mathrm{H} & 3.84161 & -4.63279 & 0.99471 \\ \mathrm{O} & 4.21559 & -6.31693 & -1.85942 \\ \mathrm{O} & 6.30358 & -5.4431 & -2.12315 \\ \mathrm{C} & 5.39507 & -6.40948 & -2.65973 \\ \mathrm{C} & 6.00812 & -7.80972 & -2.55454 \\ \mathrm{H} & 5.3164 & -8.561 & -2.94913 \\ \mathrm{H} & 6.94337 & -7.85724 & -3.12106 \\ \mathrm{H} & 6.22817 & -8.05521 & -1.51037 \\ \mathrm{C} & 5.04263 & -6.02516 & -4.08904 \\ \mathrm{H} & 5.93745 & -6.05168 & -4.71789 \\ \mathrm{H} & 4.3041 & -6.7217 & -4.49774 \\ \mathrm{H} & 4.62791 & -5.01398 & -4.10826 \\ \mathrm{C} & 2.13478 & -4.96402 & -0.30908 \\ \mathrm{H} & 1.83197 & -4.41572 & -1.20981 \\ \mathrm{H} & 1.92347 & -6.01884 & -0.51551 \\ \mathrm{C} & 1.29981 & -4.47383 & 0.89759 \\ \mathrm{H} & 1.37087 & -5.23241 & 1.69392 \\ \mathrm{H} & 1.7556 & -3.56133 & 1.29678 \\ \mathrm{C} & -0.15867 & -4.19142 & 0.59408 \\ \mathrm{C} & -0.65999 & -2.9813 & 0.89102 \\ \mathrm{H} & -1.93957 & 0.71134 & 1.33 \\ \mathrm{C} & -1.48092 & 1.69578 & 1.45744 \\ \mathrm{H} & -3.02972 & 0.8195 & 1.34121 \\ \mathrm{H} & -1.66144 & 0.07224 & 2.16919 \\ \mathrm{H} & -1.80194 & 0.92879 & -1.11966 \\ \mathrm{O} & -2.93596 & 2.11174 & -1.46513 \\ \mathrm{Si} & -2.61574 & 2.50756 & -3.27679 \\ \mathrm{C} & -28772 & 3.29627 & -3.63718 \\ \mathrm{H} & & & \\ \mathrm{H} & -3.8426 & 2.84893 & -3.42661 \\ & & \end{array}$




\begin{tabular}{|c|c|c|c|}
\hline $\mathrm{H}$ & -2.76645 & 1.62366 & -3.90689 \\
\hline $\mathrm{C}$ & -4.71074 & 1.48583 & -1.25888 \\
\hline $\mathrm{H}$ & -4.88565 & 0.58346 & -1.85671 \\
\hline $\mathrm{H}$ & -4.96401 & 1.25347 & -0.2177 \\
\hline $\mathrm{H}$ & -5.42172 & 2.24895 & -1.60144 \\
\hline $\mathrm{C}$ & -2.69749 & 3.67179 & -0.42442 \\
\hline $\mathrm{H}$ & -2.97505 & 3.52799 & 0.62542 \\
\hline $\mathrm{H}$ & -1.65489 & 4.00967 & -0.45325 \\
\hline $\mathrm{H}$ & -3.31862 & 4.48574 & -0.82028 \\
\hline C & -4.45914 & -3.78108 & 2.15754 \\
\hline C & -3.67078 & -4.73207 & 2.81815 \\
\hline C & -5.6849 & -4.1787 & 1.61254 \\
\hline $\mathrm{C}$ & -4.10449 & -6.05265 & 2.93383 \\
\hline $\mathrm{H}$ & -2.71069 & -4.42782 & 3.22431 \\
\hline $\mathrm{C}$ & -6.12476 & -5.49847 & 1.73418 \\
\hline $\mathrm{H}$ & -6.30161 & -3.45088 & 1.08836 \\
\hline $\mathrm{C}$ & -5.33449 & -6.43945 & 2.3956 \\
\hline $\mathrm{H}$ & -3.48212 & -6.78191 & 3.44631 \\
\hline $\mathrm{H}$ & -7.07905 & -5.79219 & 1.30455 \\
\hline $\mathrm{H}$ & -5.67179 & -7.46855 & 2.48694 \\
\hline C & 4.25197 & -3.52953 & -0.79954 \\
\hline $\mathrm{H}$ & 3.99827 & -3.59555 & -1.86406 \\
\hline O & 5.67189 & -3.73642 & -0.63764 \\
\hline $\mathrm{C}$ & 3.84478 & -2.21047 & -0.22789 \\
\hline $\mathrm{H}$ & 4.08884 & -2.0822 & 0.82737 \\
\hline $\mathrm{C}$ & 3.16495 & -1.23992 & -0.85724 \\
\hline $\mathrm{C}$ & 2.7856 & -1.20645 & -2.31483 \\
\hline $\mathrm{H}$ & 1.69572 & -1.24153 & -2.4322 \\
\hline $\mathrm{H}$ & 3.12198 & -0.26082 & -2.75286 \\
\hline $\mathrm{H}$ & 3.22264 & -2.02726 & -2.8859 \\
\hline ○ & -2.60751 & -2.16538 & 1.96854 \\
\hline C & -4.01292 & -2.33021 & 2.08197 \\
\hline I & -4.28127 & -1.80919 & 3.00971 \\
\hline $\mathrm{H}$ & -4.54656 & -1.82029 & 1.26491 \\
\hline C & -2.05916 & -2.47928 & 0.66812 \\
\hline $\mathrm{H}$ & -2.66844 & -3.2742 & 0.22088 \\
\hline C & 2.99536 & 2.3225 & 0.07598 \\
\hline $\mathrm{H}$ & 3.42709 & 2.26109 & 1.08791 \\
\hline $\mathrm{H}$ & 3.4864 & 3.13068 & -0.47171 \\
\hline $\mathrm{H}$ & 1.92245 & 2.54207 & 0.16363 \\
\hline C & -2.16734 & -1.27627 & -0.3011 \\
\hline $\mathrm{H}$ & -3.23115 & -1.04006 & -0.43008 \\
\hline $\mathrm{H}$ & -1.80448 & -1.60493 & -1.28137 \\
\hline 0 & 3.23076 & 1.13563 & -0.65958 \\
\hline C & 2.68969 & -0.03625 & -0.05079 \\
\hline $\mathrm{H}$ & 3.07507 & -0.11879 & 0.9793 \\
\hline C & -0.92941 & -5.33446 & -0.02182 \\
\hline $\mathrm{H}$ & -2.00539 & -5.15467 & -0.07096 \\
\hline H & -0.57712 & -5.55008 & -1.03914 \\
\hline 0 & -0.72755 & -6.52923 & 0.73757 \\
\hline & -1.11845 & -7.27347 & 0.27398 \\
\hline
\end{tabular}




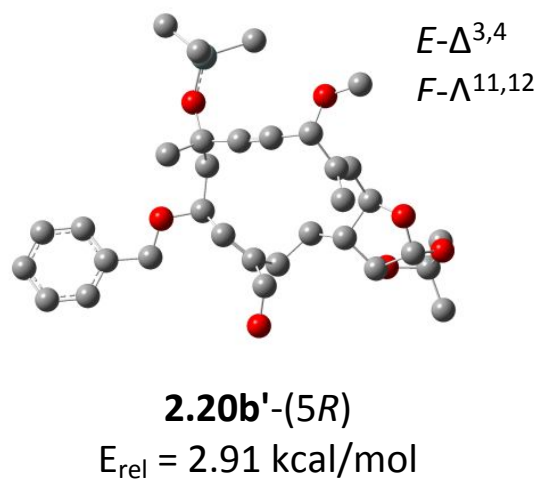

C

C

C

C

C

$\mathrm{H}$

$\mathrm{H}$

C

C

$\mathrm{H}$

C

C

$\mathrm{H}$

$\mathrm{H}$

C

$\mathrm{H}$

0

O

C

C

$\mathrm{H}$

$\mathrm{H}$

$\mathrm{H}$

C

$\mathrm{H}$

$\mathrm{H}$

$\mathrm{H}$

C

$\mathrm{H}$

$\mathrm{H}$

C

$\mathrm{H}$

$\mathrm{H}$

C

\begin{tabular}{|c|c|c|}
\hline-1.01223 & 1.99669 & 0.64412 \\
\hline 0.02952 & 2.43761 & 1.07614 \\
\hline-2.25917 & 1.53819 & -0.00727 \\
\hline 1.34319 & 2.95257 & 1.48729 \\
\hline-1.96175 & 0.32262 & -0.93888 \\
\hline-2.75759 & 0.33008 & -1.69037 \\
\hline-1.02406 & 0.53822 & -1.46197 \\
\hline 2.38262 & 1.81423 & 1.43664 \\
\hline 2.83665 & 1.43474 & 0.23465 \\
\hline 2.47367 & 1.99823 & -0.6228 \\
\hline 4.2275 & -2.06303 & 0.16125 \\
\hline 5.3958 & -1.14218 & 0.61 \\
\hline 4.09398 & -2.94218 & 0.80494 \\
\hline 5.84491 & -1.37492 & 1.584 \\
\hline 3.01733 & -1.1196 & $0.14 \varepsilon$ \\
\hline 2.64307 & -1.06643 & 1.1750 \\
\hline 4.58364 & -2.45769 & -1.15891 \\
\hline 6.37302 & -1.2714 & -0.4011 \\
\hline 5.99672 & -2.31079 & -1.30883 \\
\hline 6.71401 & -3.61238 & -0.936 \\
\hline 6.4210 & -4.41796 & -1.61768 \\
\hline 7.79855 & -3.47709 & -0.9941 \\
\hline 6.45996 & -3.91302 & 0.08504 \\
\hline $6.289^{\circ}$ & -1.85932 & -2.73144 \\
\hline 7.36335 & -1.69378 & -2.8606 \\
\hline 5.96272 & -2.62135 & -3.44548 \\
\hline 5.76025 & -0.92572 & -2.93851 \\
\hline 1.8642 & -1.4854 & -0.79331 \\
\hline 1.28 & -0.57808 & -1.00301 \\
\hline 2.280 & -1.83169 & -1.74575 \\
\hline 0.8906 & -2.55864 & -0.24104 \\
\hline 1.4588 & -3.45732 & 0.03477 \\
\hline 0.23 & -2.85912 & -1.0649 \\
\hline 0.0 & -2.10164 & 0.94 \\
\hline
\end{tabular}




\begin{tabular}{|c|c|c|c|}
\hline C & -1.90514 & -1.12159 & -0.40392 \\
\hline C & -1.09514 & -1.41691 & 0.83785 \\
\hline $\mathrm{H}$ & -1.5941 & -1.12088 & 1.75996 \\
\hline C & -3.36773 & 1.27693 & 1.02658 \\
\hline $\mathrm{H}$ & -3.57985 & 2.20108 & 1.57218 \\
\hline $\mathrm{H}$ & -4.27239 & 0.947 & 0.51114 \\
\hline $\mathrm{H}$ & -3.08203 & 0.50596 & 1.74537 \\
\hline O & -2.7384 & 2.54468 & -0.91284 \\
\hline C & 2.73156 & 1.20747 & 2.76928 \\
\hline $\mathrm{H}$ & 3.53867 & 0.47979 & 2.68783 \\
\hline $\mathrm{H}$ & 3.05277 & 1.98913 & 3.47146 \\
\hline $\mathrm{H}$ & 1.85356 & 0.72446 & 3.22113 \\
\hline $\mathrm{Si}$ & -2.58946 & 4.22025 & -1.00643 \\
\hline $\mathrm{C}$ & -4.01626 & 4.70655 & -2.13924 \\
\hline $\mathrm{H}$ & -4.02451 & 5.78837 & -2.32249 \\
\hline $\mathrm{H}$ & -3.93727 & 4.20481 & -3.11062 \\
\hline $\mathrm{H}$ & -4.98327 & 4.43281 & -1.70181 \\
\hline C & -2.79345 & 5.05951 & 0.67376 \\
\hline $\mathrm{H}$ & -3.75484 & 4.80766 & 1.13703 \\
\hline $\mathrm{H}$ & -1.99549 & 4.77466 & 1.36681 \\
\hline $\mathrm{H}$ & -2.75966 & 6.15042 & 0.55624 \\
\hline $\mathrm{C}$ & -0.95529 & 4.72205 & -1.80086 \\
\hline $\mathrm{H}$ & -0.09754 & 4.5065 & -1.15582 \\
\hline $\mathrm{H}$ & -0.80989 & 4.196 & -2.75228 \\
\hline $\mathrm{H}$ & -0.95327 & 5.79877 & -2.01729 \\
\hline $\mathrm{H}$ & 1.28697 & 3.32776 & 2.5242 \\
\hline O & 1.65003 & 4.04543 & 0.6213 \\
\hline C & 2.76777 & 4.80585 & 1.05298 \\
\hline $\mathrm{H}$ & 2.87419 & 5.62903 & 0.34279 \\
\hline $\mathrm{H}$ & 2.60404 & 5.21984 & 2.06036 \\
\hline $\mathrm{H}$ & 3.6899 & 4.20976 & 1.05733 \\
\hline O & 4.88979 & 0.16807 & 0.70355 \\
\hline C & 3.69712 & 0.24812 & -0.10802 \\
\hline $\mathrm{H}$ & 3.99458 & 0.31739 & -1.164 \\
\hline $\mathrm{H}$ & -1.51557 & -1.72384 & -1.24124 \\
\hline O & -3.26853 & -1.51668 & -0.17414 \\
\hline C & -3.46705 & -2.91849 & -0.07684 \\
\hline $\mathrm{H}$ & -3.01672 & -3.41158 & -0.95824 \\
\hline $\mathrm{H}$ & -2.95943 & -3.33153 & 0.80727 \\
\hline $\mathrm{C}$ & -4.94732 & -3.2202 & -0.01711 \\
\hline C & -5.41354 & -4.30523 & 0.73317 \\
\hline C & -5.86738 & -2.45341 & -0.74248 \\
\hline C & -6.77143 & -4.62821 & 0.752 \\
\hline $\mathrm{H}$ & -4.70931 & -4.90111 & 1.3104 \\
\hline $\mathrm{C}$ & -7.22584 & -2.76869 & -0.71723 \\
\hline $\mathrm{H}$ & -5.51079 & -1.60211 & -1.31338 \\
\hline $\mathrm{C}$ & -7.68241 & -3.85911 & 0.02669 \\
\hline $\mathrm{H}$ & -7.11746 & -5.47388 & 1.3408 \\
\hline $\mathrm{H}$ & -7.93003 & -2.16137 & -1.28028 \\
\hline $\mathrm{H}$ & -8.74113 & -4.1042 & 0.04477 \\
\hline $\mathrm{C}$ & 0.56479 & -2.48757 & 2.32043 \\
\hline
\end{tabular}




$\begin{array}{rrl}-0.08698 & -2.10802 & 3.11362 \\ 1.58082 & -2.12555 & 2.51885 \\ 0.61921 & -3.91141 & 2.44131 \\ 0.65168 & -4.15318 & 3.3698\end{array}$

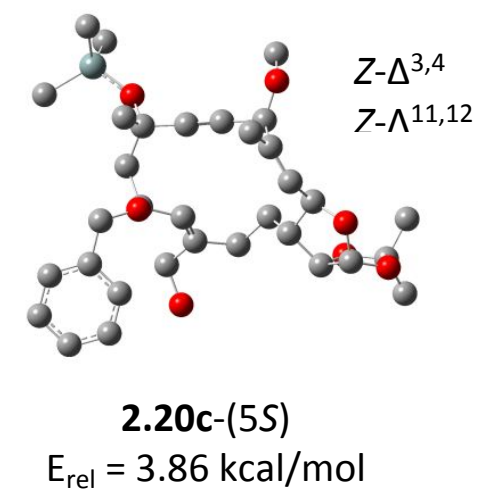

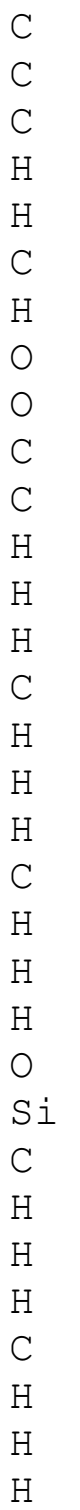

$\begin{array}{llc}2.752 & -1.26455 & 0.0088 \\ -4.45323 & 1.00443 & -0.34172 \\ -5.31927 & 0.68635 & 0.88269 \\ -4.40786 & 2.07675 & -0.57167 \\ -5.45501 & 1.49537 & 1.60649 \\ -3.08231 & 0.3898 & -0.02904 \\ -2.50878 & 1.13394 & 0.54018 \\ -5.1163 & 0.30216 & -1.39207 \\ -6.58811 & 0.39437 & 0.36209 \\ -6.45863 & -0.02419 & -1.00211 \\ -7.45225 & 0.77568 & -1.83955 \\ -7.36851 & 0.49882 & -2.89511 \\ -8.47568 & 0.58063 & -1.50403 \\ -7.24834 & 1.84556 & -1.73714 \\ -6.6543 & -1.53355 & -1.11938 \\ -7.67576 & -1.80445 & -0.8344 \\ -6.47769 & -1.86116 & -2.14907 \\ -5.95964 & -2.05096 & -0.45309 \\ 3.44365 & -1.1412 & 1.38096 \\ 3.48539 & -2.11848 & 1.86924 \\ 4.46407 & -0.7592 & 1.26102 \\ 2.88515 & -0.4475 & 2.0119 \\ 3.42256 & -2.21482 & -0.83336 \\ 5.00729 & -2.71264 & -1.05541 \\ 4.9101 & -3.81725 & -2.57644 \\ 5.89106 & -4.24052 & -2.82552 \\ 4.21751 & -4.65037 & -2.41137 \\ 4.5537 & -3.25851 & -3.44929 \\ 6.17939 & -1.26519 & -1.39282 \\ 5.84001 & -0.66648 & -2.24615 \\ 6.28548 & -0.59323 & -0.53265 \\ 7.18172 & -1.64274 & -1.63332\end{array}$




\begin{tabular}{|c|c|c|c|}
\hline C & 5.65583 & -3.712 & 0.41201 \\
\hline $\mathrm{H}$ & 5.81196 & -3.09822 & 1.30584 \\
\hline $\mathrm{H}$ & 4.96192 & -4.51821 & 0.67738 \\
\hline $\mathrm{H}$ & 6.61834 & -4.17472 & 0.15817 \\
\hline C & -3.45297 & -0.78156 & 0.92624 \\
\hline $\mathrm{H}$ & -3.60937 & -1.69389 & 0.33355 \\
\hline O & -4.70868 & -0.41095 & 1.53883 \\
\hline $\mathrm{C}$ & 2.76596 & 0.08841 & -0.7661 \\
\hline $\mathrm{H}$ & 3.81173 & 0.42217 & -0.76115 \\
\hline $\mathrm{H}$ & 2.5192 & -0.14149 & -1.80777 \\
\hline $\mathrm{C}$ & -1.4612 & -1.87346 & 2.17062 \\
\hline C & -2.48897 & -1.02048 & 2.05989 \\
\hline $\mathrm{H}$ & -2.70907 & -0.38589 & 2.91788 \\
\hline C & -0.65779 & -1.97394 & 3.44364 \\
\hline $\mathrm{H}$ & 0.40215 & -1.76156 & 3.25928 \\
\hline $\mathrm{H}$ & -1.02679 & -1.27343 & 4.19834 \\
\hline $\mathrm{H}$ & -0.71451 & -2.98969 & 3.84991 \\
\hline C & -1.66544 & 1.07769 & -2.09678 \\
\hline $\mathrm{H}$ & -2.32895 & 1.95679 & -2.1009 \\
\hline $\mathrm{H}$ & -1.63734 & 0.75756 & -3.14942 \\
\hline C & 1.39594 & -1.80642 & 0.24286 \\
\hline $\mathrm{C}$ & 0.32513 & -2.27732 & 0.5578 \\
\hline C & -0.98529 & -2.77301 & 1.03675 \\
\hline $\mathrm{H}$ & -2.99021 & -0.61304 & -1.9063 \\
\hline C & -2.29521 & -0.05753 & -1.26938 \\
\hline $\mathrm{H}$ & -1.51101 & -0.76326 & -0.97709 \\
\hline $\mathrm{C}$ & 1.85947 & 1.27892 & -0.34354 \\
\hline $\mathrm{H}$ & 2.23375 & 2.1455 & -0.90177 \\
\hline $\mathrm{C}$ & 0.40827 & 1.05516 & -0.67528 \\
\hline $\mathrm{H}$ & -0.09618 & 0.38821 & 0.01363 \\
\hline $\mathrm{C}$ & -0.25303 & 1.52182 & -1.74532 \\
\hline O & 1.94672 & 1.57379 & 1.06316 \\
\hline C & 3.01406 & 2.43707 & 1.42549 \\
\hline $\mathrm{H}$ & 3.08249 & 2.35374 & 2.51752 \\
\hline $\mathrm{H}$ & 3.97379 & 2.08118 & 1.01916 \\
\hline $\mathrm{C}$ & 2.79798 & 3.8895 & 1.0326 \\
\hline C & 3.89193 & 4.7161 & 0.75254 \\
\hline $\mathrm{C}$ & 1.50886 & 4.43518 & 0.99591 \\
\hline C & 3.70642 & 6.06687 & 0.45292 \\
\hline $\mathrm{H}$ & 4.89792 & 4.30064 & 0.76912 \\
\hline $\mathrm{C}$ & 1.32071 & 5.78253 & 0.68695 \\
\hline $\mathrm{H}$ & 0.65921 & 3.78948 & 1.19675 \\
\hline $\mathrm{C}$ & 2.41844 & 6.60355 & 0.41836 \\
\hline $\mathrm{H}$ & 4.56611 & 6.69618 & 0.23748 \\
\hline $\mathrm{H}$ & 0.31472 & 6.19348 & 0.65728 \\
\hline $\mathrm{H}$ & 2.27039 & 7.65333 & 0.17926 \\
\hline $\mathrm{C}$ & 0.3279 & 2.47223 & -2.76895 \\
\hline $\mathrm{H}$ & 1.24994 & 2.95803 & -2.44132 \\
\hline $\mathrm{H}$ & 0.53928 & 1.95213 & -3.71405 \\
\hline $\mathrm{H}$ & -1.70485 & -2.75113 & 0.20324 \\
\hline 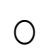 & -0.90487 & -4.10444 & 1.54185 \\
\hline
\end{tabular}




$\begin{array}{rlr}-0.57644 & -5.0652 & 0.55368 \\ -1.32322 & -5.08107 & -0.25635 \\ 0.4133 & -4.87681 & 0.11526 \\ -0.57267 & -6.0372 & 1.05313 \\ -0.61489 & 3.50204 & -3.07802 \\ -0.37562 & 3.91629 & -3.91033\end{array}$

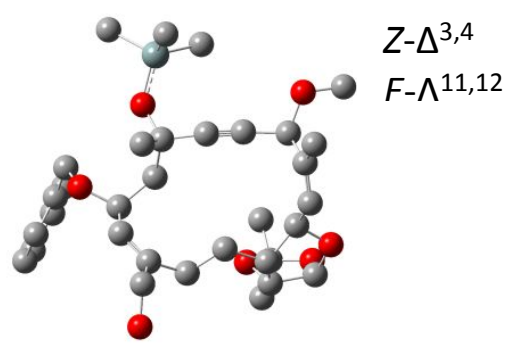

2.20c'-(5S)

$E_{\mathrm{rel}}=4.78 \mathrm{kcal} / \mathrm{mol}$

$\mathrm{C}$
$\mathrm{C}$
$\mathrm{C}$
$\mathrm{C}$
$\mathrm{H}$
$\mathrm{H}$
$\mathrm{C}$
$\mathrm{C}$
$\mathrm{H}$
$\mathrm{H}$
$\mathrm{C}$
$\mathrm{H}$
$\mathrm{O}$
$\mathrm{O}$
$\mathrm{C}$
$\mathrm{C}$
$\mathrm{H}$
$\mathrm{H}$
$\mathrm{H}$
$\mathrm{C}$
$\mathrm{H}$
$\mathrm{H}$
$\mathrm{H}$
$\mathrm{C}$
$\mathrm{H}$
$\mathrm{H}$
$\mathrm{C}$
$\mathrm{H}$
$\mathrm{H}$
$\mathrm{C}$

$$
\begin{array}{rrr}
0.81265 & 1.87182 & 0.29841 \\
1.99079 & 1.78207 & 0.03155 \\
-0.62521 & 1.88095 & 0.65387 \\
-1.17938 & 0.44869 & 0.32277 \\
-1.57312 & 0.52072 & -0.69591 \\
-0.35196 & -0.25985 & 0.25853 \\
2.08586 & -3.43215 & 0.17023 \\
3.32732 & -3.53402 & -0.75792 \\
1.81142 & -4.38475 & 0.64005 \\
3.94037 & -4.43283 & -0.64925 \\
2.48349 & -2.33838 & 1.16605 \\
3.16387 & -2.82293 & 1.88123 \\
1.05334 & -2.98101 & -0.70453 \\
2.80373 & -3.49429 & -2.07151 \\
1.37481 & -3.39986 & -2.03257 \\
0.76301 & -4.77219 & -2.32816 \\
-0.32971 & -4.71995 & -2.28913 \\
1.06591 & -5.11492 & -3.32243 \\
1.10269 & -5.51037 & -1.5944 \\
0.91251 & -2.32454 & -3.0045 \\
1.19049 & -2.59773 & -4.02678 \\
-0.17459 & -2.2094 & -2.95444 \\
1.38328 & -1.37047 & -2.75339 \\
1.39217 & -1.56368 & 1.93478 \\
1.80056 & -1.30025 & 2.91783 \\
1.22279 & -0.60775 & 1.43337 \\
0.02584 & -2.26384 & 2.12738 \\
0.185 & -3.26013 & 2.56502 \\
-0.4363 & -2.42515 & 1.14908 \\
-0.8847 & -1.46653 & 3.04373
\end{array}
$$




\begin{tabular}{|c|c|c|c|}
\hline C & -2.24525 & -0.21197 & 1.22918 \\
\hline $\mathrm{C}$ & -1.81114 & -0.58523 & 2.6356 \\
\hline $\mathrm{H}$ & -2.38832 & -0.06348 & 3.39689 \\
\hline C & -0.78748 & 2.30704 & 2.12421 \\
\hline $\mathrm{H}$ & -0.37066 & 3.30803 & 2.26453 \\
\hline $\mathrm{H}$ & -1.84989 & 2.31904 & 2.37522 \\
\hline $\mathrm{H}$ & -0.26862 & 1.61645 & 2.79349 \\
\hline 0 & -1.36401 & 2.80181 & -0.1653 \\
\hline C & -4.1967 & 0.85487 & 0.25671 \\
\hline $\mathrm{H}$ & -3.60246 & 1.36103 & -0.51417 \\
\hline $\mathrm{H}$ & -4.9509 & 1.573 & 0.6005 \\
\hline $\mathrm{Si}$ & -0.91619 & 4.25815 & -0.88961 \\
\hline $\mathrm{C}$ & -2.56753 & 4.99377 & -1.42088 \\
\hline $\mathrm{H}$ & -2.42814 & 5.96166 & -1.91818 \\
\hline $\mathrm{H}$ & -3.08903 & 4.33201 & -2.12225 \\
\hline $\mathrm{H}$ & -3.2269 & 5.151 & -0.55968 \\
\hline C & -0.04293 & 5.41757 & 0.31926 \\
\hline $\mathrm{H}$ & -0.66893 & 5.64035 & 1.19112 \\
\hline $\mathrm{H}$ & 0.90318 & 4.99521 & 0.67557 \\
\hline $\mathrm{H}$ & 0.18898 & 6.37018 & -0.17445 \\
\hline C & 0.17906 & 3.98159 & -2.4027 \\
\hline $\mathrm{H}$ & 1.17811 & 3.63416 & -2.1209 \\
\hline $\mathrm{H}$ & -0.26014 & 3.24094 & -3.08177 \\
\hline $\mathrm{H}$ & 0.29361 & 4.91818 & -2.96418 \\
\hline $\mathrm{C}$ & -4.88023 & -0.37367 & -0.3167 \\
\hline C & -5.4798 & -1.31379 & 0.53277 \\
\hline C & -4.95982 & -0.56761 & -1.69982 \\
\hline C & -6.1483 & -2.41893 & 0.00821 \\
\hline $\mathrm{H}$ & -5.40726 & -1.17371 & 1.60792 \\
\hline C & -5.63688 & -1.66892 & -2.22908 \\
\hline $\mathrm{H}$ & -4.49086 & 0.15135 & -2.36882 \\
\hline C & -6.23232 & -2.598 & -1.37547 \\
\hline $\mathrm{H}$ & -6.60733 & -3.14127 & 0.6786 \\
\hline $\mathrm{H}$ & -5.69185 & -1.80374 & -3.30638 \\
\hline $\mathrm{H}$ & -6.75606 & -3.45835 & -1.78394 \\
\hline C & 4.21388 & -0.45625 & 0.9522 \\
\hline $\mathrm{H}$ & 4.83418 & -0.86032 & 1.75299 \\
\hline $\mathrm{C}$ & 4.19973 & 0.87163 & 0.75578 \\
\hline C & 4.94645 & 1.81905 & 1.65891 \\
\hline $\mathrm{H}$ & 5.68233 & 2.41887 & 1.11292 \\
\hline $\mathrm{H}$ & 4.2512 & 2.53165 & 2.12121 \\
\hline $\mathrm{H}$ & 5.46218 & 1.27393 & 2.45486 \\
\hline O & 4.15498 & -2.42856 & -0.4668 \\
\hline C & 3.3575 & -1.45475 & 0.24192 \\
\hline $\mathrm{H}$ & 2.686 & -0.95619 & -0.46696 \\
\hline $\mathrm{H}$ & -2.5337 & -1.13342 & 0.69583 \\
\hline O & -3.41658 & 0.5945 & 1.41769 \\
\hline C & -0.67055 & -1.70428 & 4.52207 \\
\hline $\mathrm{H}$ & -1.30377 & -1.05697 & 5.13657 \\
\hline $\mathrm{H}$ & 0.37424 & -1.52825 & 4.81404 \\
\hline O & 3.95563 & 2.76683 & -0.80068 \\
\hline
\end{tabular}




$\begin{array}{llll}\mathrm{C} & 5.04985 & 2.58919 & -1.68647 \\ \mathrm{H} & 4.74268 & 2.05998 & -2.60133 \\ \mathrm{H} & 5.39994 & 3.58953 & -1.95218 \\ \mathrm{H} & 5.87565 & 2.03057 & -1.22282 \\ \mathrm{C} & 3.3811 & 1.53149 & -0.37179 \\ \mathrm{H} & 3.35883 & 0.84681 & -1.23363 \\ \mathrm{O} & -0.96092 & -3.06164 & 4.8658 \\ \mathrm{H} & -1.03203 & -3.14246 & 5.81975\end{array}$

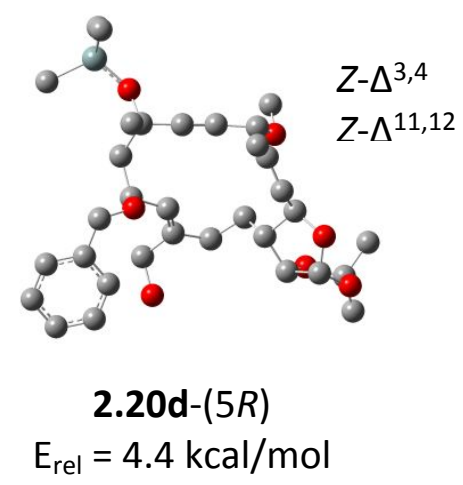

C

C

C

$\mathrm{H}$

$\mathrm{H}$

C

$\mathrm{H}$

O

O

C

C

$\mathrm{H}$

$\mathrm{H}$

$\mathrm{H}$

C

$\mathrm{H}$

$\mathrm{H}$

$\mathrm{H}$

C

$\mathrm{H}$

$\mathrm{H}$

$\mathrm{H}$

O

$\mathrm{Si}$

C

$\mathrm{H}$

$\mathrm{H}$

$\mathrm{H}$

$\begin{array}{llr}2.87773 & -1.2829 & 0.15199 \\ -4.4018 & 0.90006 & -0.26875 \\ -5.24616 & 0.63042 & 0.98222 \\ -4.33787 & 1.96578 & -0.52406 \\ -5.35658 & 1.46411 & 1.68236 \\ -3.03697 & 0.26383 & 0.02861 \\ -2.44219 & 1.01077 & 0.57284 \\ -5.10506 & 0.19438 & -1.29099 \\ -6.53231 & 0.34562 & 0.49498 \\ -6.43625 & -0.1183 & -0.85589 \\ -7.45001 & 0.65433 & -1.69522 \\ -7.39205 & 0.34258 & -2.74279 \\ -8.46515 & 0.47154 & -1.32875 \\ -7.24291 & 1.72665 & -1.63316 \\ -6.63703 & -1.6309 & -0.91969 \\ -7.64997 & -1.89044 & -0.59588 \\ -6.49178 & -1.99039 & -1.9437 \\ -5.92263 & -2.12891 & -0.25977 \\ 3.57465 & -1.04091 & 1.50631 \\ 3.66461 & -1.98163 & 2.05625 \\ 4.57588 & -0.62347 & 1.34919 \\ 2.99192 & -0.33188 & 2.09675 \\ 3.58023 & -2.2563 & -0.63517 \\ 5.1753 & -2.73386 & -0.82182 \\ 5.11391 & -3.90096 & -2.29746 \\ 6.10588 & -4.31201 & -2.52229 \\ 4.43898 & -4.74209 & -2.10231 \\ 4.75163 & -3.38699 & -3.19503\end{array}$




\begin{tabular}{|c|c|c|c|}
\hline C & 6.32774 & -1.28182 & -1.20212 \\
\hline $\mathrm{H}$ & 5.98658 & -0.71901 & -2.07886 \\
\hline $\mathrm{H}$ & 6.41566 & -0.57865 & -0.36528 \\
\hline $\mathrm{H}$ & 7.33767 & -1.65215 & -1.42087 \\
\hline C & 5.82093 & -3.66581 & 0.69103 \\
\hline $\mathrm{H}$ & 5.95076 & -3.01737 & 1.56442 \\
\hline $\mathrm{H}$ & 5.13796 & -4.47534 & 0.97427 \\
\hline $\mathrm{H}$ & 6.796 & -4.11867 & 0.46938 \\
\hline C & -3.41033 & -0.87979 & 1.01699 \\
\hline $\mathrm{H}$ & -3.59428 & -1.80523 & 0.46695 \\
\hline O & -4.64088 & -0.45387 & 1.65765 \\
\hline C & 2.82789 & 0.01403 & -0.71051 \\
\hline $\mathrm{H}$ & 3.86052 & 0.38483 & -0.74331 \\
\hline $\mathrm{H}$ & 2.5779 & -0.29441 & -1.73093 \\
\hline $\mathrm{C}$ & -1.36481 & -1.89535 & 2.29623 \\
\hline C & -2.42387 & -1.08868 & 2.13908 \\
\hline $\mathrm{H}$ & -2.65359 & -0.43515 & 2.98087 \\
\hline C & -0.57361 & -1.86496 & 3.58602 \\
\hline $\mathrm{H}$ & 0.47696 & -1.60543 & 3.40793 \\
\hline $\mathrm{H}$ & -0.99063 & -1.13692 & 4.28689 \\
\hline $\mathrm{H}$ & -0.58098 & -2.84901 & 4.0758 \\
\hline $\mathrm{C}$ & -1.65443 & 0.9011 & -2.07448 \\
\hline $\mathrm{H}$ & -2.32492 & 1.77446 & -2.10681 \\
\hline $\mathrm{H}$ & -1.62013 & 0.5522 & -3.11833 \\
\hline C & 1.55012 & -1.8635 & 0.4417 \\
\hline C & 0.50427 & -2.36273 & 0.79312 \\
\hline C & -0.7938 & -2.88195 & 1.28101 \\
\hline $\mathrm{H}$ & -0.59872 & -3.83239 & 1.81299 \\
\hline $\mathrm{H}$ & -2.99381 & -0.7683 & -1.83357 \\
\hline C & -2.27847 & -0.21412 & -1.21832 \\
\hline $\mathrm{H}$ & -1.51193 & -0.93587 & -0.92645 \\
\hline O & -1.71314 & -3.14055 & 0.23177 \\
\hline C & -1.29757 & -4.17711 & -0.6433 \\
\hline $\mathrm{H}$ & -0.35588 & -3.92597 & -1.14887 \\
\hline $\mathrm{H}$ & -1.16821 & -5.12669 & -0.10108 \\
\hline $\mathrm{H}$ & -2.09026 & -4.29332 & -1.38583 \\
\hline C & 1.88201 & 1.19566 & -0.35378 \\
\hline $\mathrm{H}$ & 2.23242 & 2.04165 & -0.95619 \\
\hline C & 0.43813 & 0.91733 & -0.67749 \\
\hline $\mathrm{H}$ & -0.04067 & 0.22782 & 0.00775 \\
\hline $\mathrm{C}$ & -0.24852 & 1.37526 & -1.73589 \\
\hline O & 1.96161 & 1.56827 & 1.03658 \\
\hline $\mathrm{C}$ & 2.9873 & 2.50128 & 1.34212 \\
\hline $\mathrm{H}$ & 3.06512 & 2.48367 & 2.43665 \\
\hline $\mathrm{H}$ & 3.96153 & 2.17017 & 0.94912 \\
\hline C & 2.69969 & 3.91712 & 0.8698 \\
\hline $\mathrm{C}$ & 3.75043 & 4.77366 & 0.52224 \\
\hline C & 1.38662 & 4.40181 & 0.82483 \\
\hline C & 3.49919 & 6.09493 & 0.14811 \\
\hline $\mathrm{H}$ & 4.77439 & 4.40479 & 0.54417 \\
\hline$C$ & 1.13281 & 5.71905 & 0.44206 \\
\hline
\end{tabular}




\begin{tabular}{llr}
0.57026 & 3.7315 & \multicolumn{1}{c}{1.0764} \\
2.18783 & 6.57095 & 0.10642 \\
4.32595 & 6.74794 & -0.11944 \\
0.10884 & 6.08236 & 0.40674 \\
1.98847 & 7.59725 & -0.19043 \\
0.29435 & 2.34805 & -2.76021 \\
1.2071 & 2.85755 & -2.44312 \\
0.50621 & 1.83895 & -3.71124 \\
-0.68001 & 3.35368 & -3.05041 \\
-0.47604 & 3.76349 & -3.89424
\end{tabular}

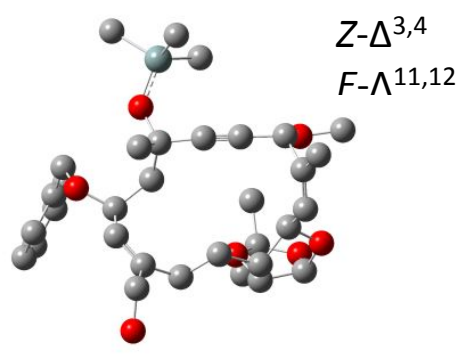

2.20d'-(5R)

$\mathrm{E}_{\mathrm{rel}}=3.85 \mathrm{kcal} / \mathrm{mol}$

$\mathrm{C}$
$\mathrm{C}$
$\mathrm{C}$
$\mathrm{C}$
$\mathrm{H}$
$\mathrm{H}$
$\mathrm{C}$
$\mathrm{C}$
$\mathrm{H}$
$\mathrm{H}$
$\mathrm{C}$
$\mathrm{H}$
$\mathrm{O}$
$\mathrm{O}$
$\mathrm{C}$
$\mathrm{C}$
$\mathrm{H}$
$\mathrm{H}$
$\mathrm{H}$
$\mathrm{C}$
$\mathrm{H}$
$\mathrm{H}$
$\mathrm{H}$
$\mathrm{C}$
$\mathrm{H}$
$\mathrm{H}$

$\begin{array}{ccc}0.79401 & 1.94923 & 0.52075 \\ 1.98568 & 1.9228 & 0.3038 \\ -0.65411 & 1.87878 & 0.82488 \\ -1.14117 & 0.44064 & 0.42693 \\ -1.50022 & 0.53162 & -0.60306 \\ -0.2876 & -0.23598 & 0.36984 \\ 2.13517 & -3.19078 & 0.04333 \\ 3.37748 & -3.1843 & -0.87864 \\ 1.85437 & -4.19196 & 0.39393 \\ 4.029 & -4.06031 & -0.82238 \\ 2.52148 & -2.21672 & 1.16179 \\ 3.22348 & -2.76719 & 1.80544 \\ 1.11313 & -2.63785 & -0.78493 \\ 2.86024 & -3.11857 & -2.18671 \\ 1.45581 & -2.82822 & -2.16338 \\ 0.69996 & -4.02886 & -2.73342 \\ -0.37849 & -3.84164 & -2.72112 \\ 1.01354 & -4.21975 & -3.76457 \\ 0.90788 & -4.9233 & -2.13761 \\ 1.17892 & -1.53388 & -2.9183 \\ 1.43603 & -1.65684 & -3.97518 \\ 0.11795 & -1.27394 & -2.84546 \\ 1.77577 & -0.71734 & -2.50329 \\ 1.42202 & -1.56614 & 2.02805 \\ 1.82813 & -1.4211 & 3.03652 \\ 1.23234 & -0.55865 & 1.64923\end{array}$




\begin{tabular}{|c|c|c|c|}
\hline C & 0.06846 & -2.3077 & 2.13149 \\
\hline $\mathrm{H}$ & 0.23761 & -3.32675 & 2.50895 \\
\hline $\mathrm{H}$ & -0.3604 & -2.41291 & 1.1307 \\
\hline C & -0.88656 & -1.58582 & 3.06446 \\
\hline C & -2.2195 & -0.2807 & 1.2698 \\
\hline C & -1.82066 & -0.70536 & 2.67216 \\
\hline $\mathrm{H}$ & -2.43342 & -0.23355 & 3.43827 \\
\hline C & -0.86705 & 2.25311 & 2.30371 \\
\hline $\mathrm{H}$ & -0.49141 & 3.26634 & 2.47569 \\
\hline $\mathrm{H}$ & -1.93506 & 2.22295 & 2.52754 \\
\hline $\mathrm{H}$ & -0.34005 & 1.56388 & 2.96822 \\
\hline O & -1.4169 & 2.81035 & 0.04019 \\
\hline C & -4.17829 & 0.75644 & 0.28387 \\
\hline $\mathrm{H}$ & -3.57826 & 1.2715 & -0.47671 \\
\hline $\mathrm{H}$ & -4.94285 & 1.46715 & 0.62014 \\
\hline Si & -0.95712 & 4.09779 & -0.94274 \\
\hline $\mathrm{C}$ & -2.60133 & 4.88577 & -1.4184 \\
\hline $\mathrm{H}$ & -2.44796 & 5.74688 & -2.08078 \\
\hline $\mathrm{H}$ & -3.24812 & 4.1737 & -1.94394 \\
\hline $\mathrm{H}$ & -3.14393 & 5.23641 & -0.53311 \\
\hline C & 0.0987 & 5.35506 & -0.00653 \\
\hline $\mathrm{H}$ & -0.43159 & 5.73592 & 0.87455 \\
\hline $\mathrm{H}$ & 1.04662 & 4.92327 & 0.33095 \\
\hline $\mathrm{H}$ & 0.33247 & 6.21394 & -0.64882 \\
\hline C & -0.06209 & 3.50377 & -2.49639 \\
\hline $\mathrm{H}$ & 0.8767 & 2.99078 & -2.26399 \\
\hline $\mathrm{H}$ & -0.69078 & 2.8082 & -3.06583 \\
\hline $\mathrm{H}$ & 0.16864 & 4.35157 & -3.15477 \\
\hline $\mathrm{C}$ & -4.84359 & -0.47473 & -0.30555 \\
\hline C & -5.43024 & -1.43391 & 0.53128 \\
\hline $\mathrm{C}$ & -4.92087 & -0.65126 & -1.69112 \\
\hline C & -6.08402 & -2.54082 & -0.00802 \\
\hline $\mathrm{H}$ & -5.35883 & -1.30726 & 1.60807 \\
\hline C & -5.58316 & -1.7543 & -2.23503 \\
\hline $\mathrm{H}$ & -4.46116 & 0.08234 & -2.35062 \\
\hline $\mathrm{C}$ & -6.16603 & -2.70255 & -1.39386 \\
\hline $\mathrm{H}$ & -6.53282 & -3.27829 & 0.65275 \\
\hline $\mathrm{H}$ & -5.63591 & -1.87582 & -3.31399 \\
\hline $\mathrm{H}$ & -6.6779 & -3.56452 & -1.81379 \\
\hline $\mathrm{C}$ & 4.21145 & -0.2753 & 1.11547 \\
\hline $\mathrm{H}$ & 4.86323 & -0.74605 & 1.85307 \\
\hline C & 4.22771 & 1.06169 & 1.00898 \\
\hline C & 5.07288 & 1.92133 & 1.914 \\
\hline $\mathrm{H}$ & 5.80681 & 2.5047 & 1.34076 \\
\hline $\mathrm{H}$ & 4.45219 & 2.64684 & 2.45788 \\
\hline $\mathrm{H}$ & 5.61335 & 1.32097 & 2.65156 \\
\hline 0 & 4.15336 & -2.0588 & -0.5112 \\
\hline C & 3.34282 & -1.20486 & 0.33123 \\
\hline $\mathrm{H}$ & 2.6531 & -0.63325 & -0.29313 \\
\hline $\mathrm{H}$ & -2.47228 & -1.18464 & 0.69058 \\
\hline 0 & -3.41223 & 0.49544 & 1.45312 \\
\hline
\end{tabular}




$\begin{array}{rrr}-0.70838 & -1.89551 & 4.53395 \\ -1.37526 & -1.29666 & 5.16197 \\ 0.32291 & -1.71036 & 4.86514 \\ 3.41083 & 1.84835 & -0.04049 \\ 3.48639 & 1.31995 & -1.36698 \\ 4.79304 & 1.34538 & -1.92391 \\ 5.21302 & 2.36378 & -1.9084 \\ 5.47331 & 0.66321 & -1.39999 \\ 4.68861 & 1.01782 & -2.96037 \\ 3.82161 & 2.87363 & -0.0585 \\ -0.97417 & -3.27562 & 4.7977 \\ -1.05743 & -3.41068 & 5.74449\end{array}$

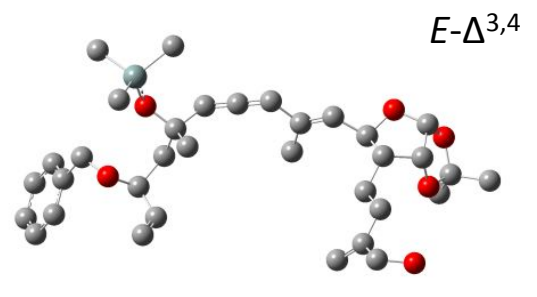

$$
\begin{gathered}
2.21 \mathrm{a}-(6 S) \\
E_{\mathrm{rel}}=0.96 \mathrm{kcal} / \mathrm{mol}
\end{gathered}
$$

$\mathrm{C}$
$\mathrm{C}$
$\mathrm{C}$
$\mathrm{C}$
$\mathrm{H}$
$\mathrm{H}$
$\mathrm{C}$
$\mathrm{H}$
$\mathrm{O}$
$\mathrm{O}$
$\mathrm{C}$
$\mathrm{C}$
$\mathrm{H}$
$\mathrm{H}$
$\mathrm{H}$
$\mathrm{C}$
$\mathrm{H}$
$\mathrm{H}$
$\mathrm{H}$
$\mathrm{C}$
$\mathrm{H}$
$\mathrm{H}$
$\mathrm{C}$
$\mathrm{H}$

$$
\begin{array}{lrr}
1.74238 & -2.43135 & \multicolumn{1}{l}{-1.7499} \\
2.24396 & -1.42825 & \multicolumn{1}{l}{0.68999} \\
-5.08779 & 0.2024 & 0.7373 \\
-6.17123 & -0.83443 & 0.50609 \\
-5.27717 & 0.89055 & 1.53443 \\
-6.63759 & -1.26638 & 1.36958 \\
-3.86224 & -0.61145 & 0.86771 \\
-3.85346 & -1.19202 & 1.76744 \\
-5.04655 & 0.87979 & -0.51758 \\
-7.13233 & -0.11624 & -0.29198 \\
-6.44463 & 1.12339 & -0.62616 \\
-6.81062 & 2.19695 & 0.41677 \\
-6.28977 & 3.10357 & 0.19384 \\
-7.86343 & 2.37582 & 0.38769 \\
-6.5331 & 1.85801 & 1.39252 \\
-6.84847 & 1.61331 & -2.02749 \\
-7.9056 & 1.78563 & -2.052 \\
-6.33251 & 2.52506 & -2.25083 \\
-6.5903 & 0.86859 & -2.75462 \\
-2.53994 & 0.17739 & 0.80178 \\
-1.72363 & -0.51342 & 0.80125 \\
-2.52903 & 0.75195 & -0.10141 \\
-2.38919 & 1.13235 & 1.99552 \\
-3.20273 & 1.82646 & 2.00969
\end{array}
$$




\begin{tabular}{|c|c|c|c|}
\hline $\mathrm{H}$ & -2.3846 & 0.57518 & 2.90987 \\
\hline C & 1.4934 & -1.675 & 0.63259 \\
\hline $\mathrm{H}$ & 1.66415 & -2.67633 & 0.96269 \\
\hline $\mathrm{H}$ & 1.8518 & -0.988 & 1.37324 \\
\hline $\mathrm{H}$ & 0.44325 & -1.52074 & 0.4842 \\
\hline 0 & 3.65528 & -1.60382 & -0.50397 \\
\hline Si & 4.29274 & -2.69155 & 0.58746 \\
\hline $\mathrm{C}$ & 3.62799 & -4.46886 & 0.19079 \\
\hline $\mathrm{H}$ & 4.04609 & -5.16841 & 0.88403 \\
\hline $\mathrm{H}$ & 2.56069 & -4.47763 & 0.2751 \\
\hline $\mathrm{H}$ & 3.90943 & -4.74013 & -0.805 \\
\hline C & 6.22878 & -2.67424 & 0.43761 \\
\hline $\mathrm{H}$ & 6.51119 & -2.94284 & -0.56 \\
\hline $\mathrm{H}$ & 6.59573 & -1.69311 & 0.65937 \\
\hline $\mathrm{H}$ & 6.64759 & -3.37625 & 1.12805 \\
\hline $\mathrm{C}$ & 3.77773 & -2.20334 & 2.39689 \\
\hline $\mathrm{H}$ & 4.14444 & -1.22283 & 2.62107 \\
\hline $\mathrm{H}$ & 2.71036 & -2.21153 & 2.48084 \\
\hline $\mathrm{H}$ & 4.19498 & -2.90682 & 3.08815 \\
\hline C & 4.60408 & 2.48978 & -0.85097 \\
\hline C & 4.19215 & 3.39723 & 0.13568 \\
\hline $\mathrm{C}$ & 4.96565 & 2.95739 & -2.12414 \\
\hline C & 4.14782 & 4.76943 & -0.14927 \\
\hline $\mathrm{H}$ & 3.91213 & 3.04571 & 1.10801 \\
\hline C & 4.92673 & 4.33197 & -2.40664 \\
\hline $\mathrm{H}$ & 5.27315 & 2.26511 & -2.87559 \\
\hline C & 4.51698 & 5.23771 & -1.41803 \\
\hline $\mathrm{H}$ & 3.83215 & 5.45934 & 0.60214 \\
\hline $\mathrm{H}$ & 5.20921 & 4.68936 & -3.37377 \\
\hline $\mathrm{H}$ & 4.48908 & 6.28576 & -1.63071 \\
\hline $\mathrm{C}$ & -4.11252 & -1.44047 & -0.38857 \\
\hline $\mathrm{H}$ & -4.08004 & -0.79742 & -1.24236 \\
\hline O & -5.45493 & -1.94411 & -0.16789 \\
\hline C & -3.06842 & -2.55663 & -0.58197 \\
\hline $\mathrm{H}$ & -3.29244 & -3.57014 & -0.31421 \\
\hline $\mathrm{C}$ & -1.85123 & -2.22556 & -1.08555 \\
\hline C & -1.50044 & -0.73953 & -1.26855 \\
\hline $\mathrm{H}$ & -1.05167 & -0.36574 & -0.37198 \\
\hline $\mathrm{H}$ & -0.81491 & -0.63499 & -2.08242 \\
\hline $\mathrm{H}$ & -2.39285 & -0.18671 & -1.48037 \\
\hline O & 3.69688 & 0.68123 & 0.47738 \\
\hline C & 4.65333 & 0.98388 & -0.52876 \\
\hline $\mathrm{H}$ & 5.6272 & 0.7316 & -0.16841 \\
\hline $\mathrm{H}$ & 4.4414 & 0.41447 & -1.41178 \\
\hline $\mathrm{C}$ & 2.35121 & 0.91848 & 0.06516 \\
\hline $\mathrm{H}$ & 2.22967 & 1.94116 & -0.21805 \\
\hline $\mathrm{C}$ & 1.98808 & 0.01071 & -1.12332 \\
\hline $\mathrm{H}$ & 2.58697 & 0.24208 & -1.98086 \\
\hline $\mathrm{H}$ & 0.95374 & 0.14523 & -1.36922 \\
\hline $\mathrm{C}$ & -0.26303 & 2.38514 & 3.05394 \\
\hline $\mathrm{H}$ & 0.78421 & 2.37748 & 2.82548 \\
\hline
\end{tabular}


$\mathrm{H}$

C

C

$\mathrm{H}$

$\mathrm{H}$

O

$\mathrm{H}$

C

$\mathrm{H}$

C

$\mathrm{H}$

$\mathrm{H}$

C

C

$\mathrm{H}$

$\mathrm{H}$

$\begin{array}{ccr}-0.44786 & 1.74221 & 3.88604 \\ 0.47607 & -2.89279 & -1.62594 \\ -0.80477 & -3.29119 & -1.45771 \\ -1.078 & -4.31983 & -1.57279 \\ 2.37868 & -2.76242 & -2.54521 \\ -0.67132 & 3.71488 & 3.38247 \\ -0.16535 & 4.02873 & 4.13545 \\ 1.41926 & 0.58154 & 1.25045 \\ 0.4477 & 0.1832 & 1.06566 \\ 1.82604 & 0.78293 & 2.52515 \\ 1.17843 & 0.51632 & 3.33375 \\ 2.78625 & 1.20889 & 2.71964 \\ -1.057 & 1.88984 & 1.83066 \\ -0.57816 & 2.12363 & 0.58291 \\ 0.345 & 2.6468 & 0.45105 \\ -1.12652 & 1.78088 & -0.27025\end{array}$

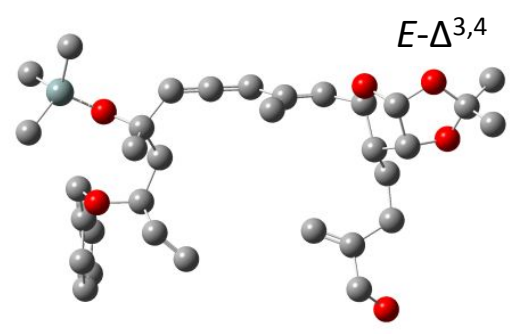

2.21b-(6R)

$\mathrm{E}_{\mathrm{rel}}=5.93 \mathrm{kcal} / \mathrm{mol}$

C

C

C

C

$\mathrm{H}$

$\mathrm{H}$

O

O

.




\begin{tabular}{|c|c|c|c|}
\hline $\mathrm{C}$ & -6.54649 & 0.33724 & 0.397 \\
\hline $\mathrm{C}$ & -6.49201 & 0.73101 & 1.88624 \\
\hline $\mathrm{H}$ & -6.7979 & 1.74827 & 1.99623 \\
\hline $\mathrm{H}$ & -7.15024 & 0.09964 & 2.44782 \\
\hline $\mathrm{H}$ & -5.49425 & 0.615 & 2.25093 \\
\hline C & -7.99645 & 0.49528 & -0.09604 \\
\hline $\mathrm{H}$ & -8.64244 & -0.13402 & 0.48279 \\
\hline $\mathrm{H}$ & -8.30185 & 1.51291 & 0.01475 \\
\hline $\mathrm{H}$ & -8.0546 & 0.21361 & -1.12609 \\
\hline C & -2.91068 & 2.21531 & -1.02764 \\
\hline $\mathrm{H}$ & -2.05813 & 2.1728 & -1.6736 \\
\hline $\mathrm{H}$ & -3.75544 & 2.57978 & -1.57427 \\
\hline $\mathrm{C}$ & -2.60954 & 3.16463 & 0.14791 \\
\hline $\mathrm{H}$ & -2.40115 & 4.14314 & -0.22887 \\
\hline $\mathrm{H}$ & -3.45462 & 3.21066 & 0.80073 \\
\hline $\mathrm{C}$ & 1.45734 & -2.24331 & 0.77241 \\
\hline $\mathrm{H}$ & 1.65656 & -3.27098 & 0.97678 \\
\hline $\mathrm{H}$ & 1.71382 & -1.65266 & 1.62684 \\
\hline $\mathrm{H}$ & 0.41771 & -2.11628 & 0.55248 \\
\hline 0 & 3.68508 & -1.96302 & -0.12964 \\
\hline $\mathrm{Si}$ & 4.17605 & -3.08737 & 1.01045 \\
\hline $\mathrm{C}$ & 3.589 & -4.85864 & 0.49334 \\
\hline $\mathrm{H}$ & 3.90884 & -5.56617 & 1.23014 \\
\hline $\mathrm{H}$ & 2.52264 & -4.87581 & 0.42134 \\
\hline $\mathrm{H}$ & 4.01383 & -5.11247 & -0.45354 \\
\hline C & 6.111 & -3.0747 & 1.14039 \\
\hline $\mathrm{H}$ & 6.53289 & -3.32656 & 0.191 \\
\hline $\mathrm{H}$ & 6.44394 & -2.10074 & 1.4317 \\
\hline $\mathrm{H}$ & 6.42275 & -3.79074 & 1.87067 \\
\hline C & 3.40622 & -2.63384 & 2.73222 \\
\hline $\mathrm{H}$ & 3.73258 & -1.65814 & 3.02384 \\
\hline $\mathrm{H}$ & 2.3388 & -2.64467 & 2.66045 \\
\hline $\mathrm{H}$ & 3.72193 & -3.34879 & 3.46279 \\
\hline C & 4.35782 & 2.22158 & -0.72368 \\
\hline C & 4.08643 & 3.17172 & 0.268 \\
\hline C & 4.45191 & 2.61882 & -2.0644 \\
\hline C & 3.92772 & 4.5208 & -0.08114 \\
\hline $\mathrm{H}$ & 4.00062 & 2.86793 & 1.29178 \\
\hline C & 4.28769 & 3.96553 & -2.41283 \\
\hline $\mathrm{H}$ & 4.64696 & 1.89088 & -2.8234 \\
\hline C & 4.03243 & 4.91752 & -1.42046 \\
\hline $\mathrm{H}$ & 3.72778 & 5.24831 & 0.67803 \\
\hline $\mathrm{H}$ & 4.35977 & 4.26942 & -3.43588 \\
\hline $\mathrm{H}$ & 3.91546 & 5.94943 & -1.68301 \\
\hline O & 3.76227 & 0.40037 & 0.7843 \\
\hline C & 4.55988 & 0.7362 & -0.35379 \\
\hline $\mathrm{H}$ & 5.59113 & 0.56578 & -0.12858 \\
\hline $\mathrm{H}$ & 4.2678 & 0.12105 & -1.18043 \\
\hline C & 2.38373 & 0.58969 & 0.46508 \\
\hline $\mathrm{H}$ & 2.21651 & 1.61681 & 0.21843 \\
\hline $\mathrm{C}$ & 2.01744 & -0.29747 & -0.745 \\
\hline
\end{tabular}




\begin{tabular}{rrr}
2.61226 & -0.00294 & \multicolumn{1}{l}{-1.58335} \\
0.9786 & -0.1663 & -0.97466 \\
-1.24861 & 2.98133 & 2.41895 \\
-0.21188 & 3.02625 & 2.68163 \\
-1.72935 & 2.22255 & 2.999 \\
0.86053 & -2.19112 & -2.4149 \\
-0.231 & -1.69671 & \multicolumn{1}{l}{-3.04035} \\
2.46045 & -3.49587 & -1.95156 \\
-0.28076 & -1.61046 & -4.10587 \\
-1.39363 & -1.26134 & -2.13174 \\
-1.375 & -1.70593 & -0.66758 \\
-0.86614 & -2.64151 & -0.58482 \\
-0.87026 & -0.97179 & -0.08175 \\
-2.38088 & -1.81561 & -0.31861 \\
-2.42575 & -0.50458 & -2.55144 \\
-2.43831 & -0.08368 & -3.53602 \\
-3.21319 & 0.80405 & -0.48965 \\
-2.35253 & 0.49391 & 0.07265 \\
-3.57947 & -0.24737 & -1.5582 \\
-4.44365 & 0.04764 & -2.11684 \\
-3.88212 & -1.40475 & -0.72853 \\
-1.86658 & 4.25054 & 2.677 \\
-1.79577 & 4.46203 & 3.61054 \\
1.50986 & 0.21899 & 1.67885 \\
1.63869 & -0.72537 & 2.16943 \\
0.57761 & 1.09806 & 2.12511 \\
0.45797 & 2.04455 & 1.63493 \\
-0.03543 & 0.85127 & 2.96587 \\
-1.38599 & 2.64664 & 0.92767 \\
-0.43362 & 1.90624 & 0.30745 \\
-0.53113 & 1.66523 & -0.73305 \\
0.41682 & 1.56089 & 0.85797
\end{tabular}

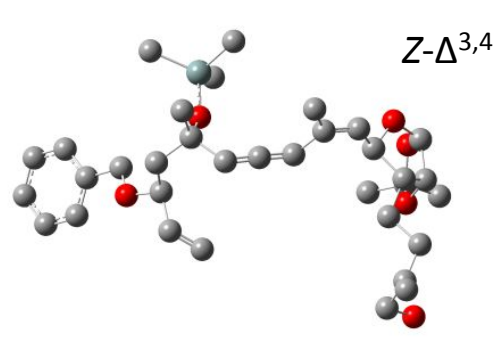

$$
\begin{gathered}
\text { 2.21c-(6S) } \\
E_{\mathrm{rel}}=1.44 \mathrm{kcal} / \mathrm{mol}
\end{gathered}
$$

C
C
H
C

$\begin{array}{lll}0 . & 0 . & 0 . \\ 1.36854 & 0 . & 0 . \\ 1.86419 & 0.96345 & 0 . \\ -1.36828 & 0.0001 & 0.00007\end{array}$




\begin{tabular}{|c|c|c|c|}
\hline $\mathrm{H}$ & -1.8972 & -0.30341 & -0.85495 \\
\hline $\mathrm{C}$ & -2.1713 & 0.46694 & 1.27296 \\
\hline C & -3.52481 & 0.28462 & 1.40972 \\
\hline $\mathrm{H}$ & -3.98262 & 0.76595 & 2.23021 \\
\hline C & -4.49428 & -0.50145 & 0.48396 \\
\hline $\mathrm{H}$ & -4.75114 & -1.43901 & 0.95721 \\
\hline C & -4.01015 & -0.82954 & -0.92876 \\
\hline $\mathrm{H}$ & -3.6433 & 0.04699 & -1.40006 \\
\hline 0 & -5.64575 & 0.31694 & 0.23403 \\
\hline C & -5.33555 & -1.29118 & -1.51334 \\
\hline $\mathrm{H}$ & -5.40255 & -1.37544 & -2.58257 \\
\hline C & -6.31562 & -0.32533 & -0.86326 \\
\hline $\mathrm{H}$ & -6.64379 & 0.42871 & -1.56168 \\
\hline C & -2.94985 & -1.94382 & -1.00048 \\
\hline $\mathrm{H}$ & -2.0944 & -1.67604 & -0.42306 \\
\hline $\mathrm{H}$ & -3.34062 & -2.87596 & -0.62456 \\
\hline 0 & -5.67147 & -2.53679 & -1.0026 \\
\hline 0 & -7.35909 & -1.16799 & -0.33487 \\
\hline C & -7.11711 & -2.43958 & -0.93493 \\
\hline $\mathrm{C}$ & -2.6044 & -2.03484 & -2.46223 \\
\hline $\mathrm{H}$ & -3.47036 & -2.35793 & -3.00454 \\
\hline $\mathrm{H}$ & -2.33472 & -1.03661 & -2.75969 \\
\hline C & -1.37701 & -2.94929 & -2.77927 \\
\hline $\mathrm{C}$ & 1.71696 & -2.65195 & -2.06527 \\
\hline $\mathrm{H}$ & 1.63959 & -3.5742 & -1.52135 \\
\hline C & 2.75357 & -1.74103 & -1.29537 \\
\hline $\mathrm{H}$ & 3.62295 & -2.35544 & -1.16233 \\
\hline $\mathrm{H}$ & 3.00384 & -0.8642 & -1.85225 \\
\hline C & 2.25864 & -1.27783 & 0.09942 \\
\hline 0 & 1.49549 & -2.36332 & 0.66423 \\
\hline $\mathrm{Si}$ & 1.36697 & -3.07672 & 2.18629 \\
\hline $\mathrm{C}$ & 1.03565 & -1.78314 & 3.60572 \\
\hline $\mathrm{H}$ & 0.92684 & -2.30465 & 4.53743 \\
\hline $\mathrm{H}$ & 1.85782 & -1.10188 & 3.67689 \\
\hline $\mathrm{H}$ & 0.14033 & -1.23716 & 3.3991 \\
\hline C & 2.99801 & -4.03522 & 2.60986 \\
\hline $\mathrm{H}$ & 2.88184 & -4.53352 & 3.5508 \\
\hline $\mathrm{H}$ & 3.19509 & -4.75526 & 1.84618 \\
\hline $\mathrm{H}$ & 3.81581 & -3.34787 & 2.67235 \\
\hline $\mathrm{C}$ & -0.13297 & -4.29888 & 2.06537 \\
\hline $\mathrm{H}$ & -1.02411 & -3.74705 & 1.83842 \\
\hline $\mathrm{H}$ & 0.05215 & -5.01007 & 1.28897 \\
\hline $\mathrm{H}$ & -0.25968 & -4.80846 & 2.99498 \\
\hline C & 3.50155 & -0.89991 & 0.95446 \\
\hline $\mathrm{H}$ & 3.19343 & -0.59174 & 1.93567 \\
\hline $\mathrm{H}$ & 4.14691 & -1.74747 & 1.03948 \\
\hline $\mathrm{H}$ & 4.02683 & -0.09484 & 0.48326 \\
\hline 0 & 2.21753 & -2.91094 & -3.38581 \\
\hline C & 3.30372 & -3.83732 & -3.32338 \\
\hline $\mathrm{H}$ & 4.1619 & -3.37441 & -2.88161 \\
\hline $\mathrm{H}$ & 3.00054 & -4.66969 & -2.73141 \\
\hline
\end{tabular}


$\begin{array}{lll}3.638 & -4.33446 & -4.75098\end{array}$

$\begin{array}{lll}4.9134 & -4.83195 & -5.08224\end{array}$

$\begin{array}{lll}2.6331 & -4.29826 & -5.72848\end{array}$

$\begin{array}{lll}5.16498 & -5.30312 & -6.38519\end{array}$

$5.68969-4.85705-4.34719$

$2.88948-4.7576 \quad-7.02645$

$\begin{array}{lll}1.66693 & -3.9164 & -5.48107\end{array}$

$\begin{array}{lll}4.15066 & -5.26677 & -7.35408\end{array}$

$\begin{array}{lll}6.12757 & -5.69384 & -6.63798\end{array}$

$\begin{array}{lll}2.11918 & -4.72259 & -7.76656\end{array}$

$\begin{array}{lll}4.34164 & -5.62698 & -8.34355\end{array}$

$\begin{array}{lll}-1.48431 & 1.14817 & 2.48261\end{array}$

$\begin{array}{lll}-0.49139 & 0.77213 & 2.60277\end{array}$

$\begin{array}{lll}-1.45126 & 2.20274 & 2.31885\end{array}$

$\begin{array}{lll}-2.04695 & 0.94344 & 3.37433\end{array}$

$\begin{array}{lll}-0.72582 & -4.12047 & -1.88241\end{array}$

$\begin{array}{lll}0.32566 & -4.19262 & -2.0963\end{array}$

$\begin{array}{lll}-0.84634 & -3.90223 & -0.83085\end{array}$

$\begin{array}{lll}-7.91913 & -2.50239 & -2.26928\end{array}$

$\begin{array}{lll}-7.73556 & -3.43192 & -2.76173\end{array}$

$\begin{array}{lll}-8.96943 & -2.42392 & -2.03932\end{array}$

$\begin{array}{lll}-7.64082 & -1.69817 & -2.91366\end{array}$

$\begin{array}{lll}-7.53936 & -3.60424 & -0.06332\end{array}$

$\begin{array}{lll}-8.5747 & -3.54469 & 0.1597\end{array}$

$\begin{array}{lll}-7.33365 & -4.5185 & -0.58109\end{array}$

$\begin{array}{lll}-6.96953 & -3.57132 & 0.84259\end{array}$

$\begin{array}{lll}0.27115 & -2.12358 & -2.22268\end{array}$

$\begin{array}{lll}-0.24642 & -2.35583 & -3.13701\end{array}$

$\begin{array}{llll}-0.89255 & -2.64504 & -4.03503\end{array}$

$\begin{array}{lll}-0.07011 & -3.15501 & -4.46463\end{array}$

$\begin{array}{lll}-1.36022 & -1.83654 & -4.58282\end{array}$

$\begin{array}{lll}-0.38437 & -1.4366 & -1.26928\end{array}$

$\begin{array}{lll}0.09803 & -1.1859 & -0.35704\end{array}$

$\begin{array}{lll}-1.40771 & -1.15429 & -1.43363\end{array}$

$\begin{array}{lll}-1.35662 & -5.37452 & -2.15507\end{array}$

$\begin{array}{lll}-0.72336 & -6.08536 & -2.03144\end{array}$

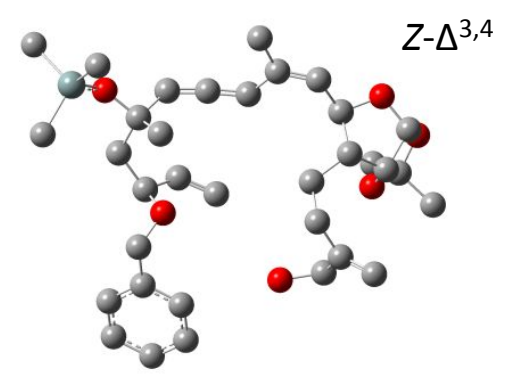

2.21d-(6R)

$\mathrm{E}_{\mathrm{rel}}=0.00 \mathrm{kcal} / \mathrm{mol}$ 


\begin{tabular}{|c|c|c|c|}
\hline $\mathrm{C}$ & -1.32764 & -2.74684 & 1.0452 \\
\hline C & -2.47031 & -1.89725 & 0.44133 \\
\hline C & 4.99748 & 0.49109 & -0.9236 \\
\hline C & 5.98164 & -0.63014 & -1.28824 \\
\hline $\mathrm{H}$ & 5.04301 & 1.3507 & -1.6045 \\
\hline $\mathrm{H}$ & 6.31889 & -0.66344 & -2.32803 \\
\hline C & 3.62618 & -0.18984 & -0.88556 \\
\hline $\mathrm{H}$ & 3.2392 & -0.21917 & -1.91378 \\
\hline O & 5.41819 & 0.86968 & 0.38648 \\
\hline 0 & 7.11727 & -0.38763 & -0.50018 \\
\hline C & 6.76333 & 0.43426 & 0.62015 \\
\hline $\mathrm{C}$ & 7.70562 & 1.63509 & 0.64717 \\
\hline $\mathrm{H}$ & 7.45411 & 2.29581 & 1.48266 \\
\hline $\mathrm{H}$ & 8.7425 & 1.30267 & 0.7593 \\
\hline $\mathrm{H}$ & 7.61774 & 2.19793 & -0.28678 \\
\hline C & 6.79796 & -0.38264 & 1.90878 \\
\hline $\mathrm{H}$ & 7.81875 & -0.71906 & 2.11552 \\
\hline $\mathrm{H}$ & 6.44857 & 0.22184 & 2.75202 \\
\hline $\mathrm{H}$ & 6.15604 & -1.26145 & 1.80733 \\
\hline $\mathrm{C}$ & 2.58825 & 0.43171 & 0.05762 \\
\hline $\mathrm{H}$ & 1.89753 & -0.36592 & 0.32078 \\
\hline $\mathrm{H}$ & 3.10299 & 0.71916 & 0.98014 \\
\hline C & 1.75889 & 1.60437 & -0.48472 \\
\hline $\mathrm{H}$ & 1.10761 & 1.27237 & -1.30172 \\
\hline $\mathrm{H}$ & 1.10117 & 1.98036 & 0.31433 \\
\hline $\mathrm{C}$ & -2.33754 & -1.8742 & -1.08789 \\
\hline $\mathrm{H}$ & -2.29852 & -2.90033 & -1.46499 \\
\hline $\mathrm{H}$ & -3.18237 & -1.3561 & -1.55085 \\
\hline $\mathrm{H}$ & -1.42639 & -1.35561 & -1.39282 \\
\hline 0 & -3.64397 & -2.62636 & 0.84581 \\
\hline $\mathrm{Si}$ & -5.10375 & -3.0935 & 0.1772 \\
\hline $\mathrm{C}$ & -5.99773 & -3.93212 & 1.60614 \\
\hline $\mathrm{H}$ & -6.98785 & -4.29068 & 1.29868 \\
\hline $\mathrm{H}$ & -5.43018 & -4.79338 & 1.9768 \\
\hline $\mathrm{H}$ & -6.13705 & -3.24131 & 2.4455 \\
\hline C & -6.13162 & -1.6219 & -0.42477 \\
\hline $\mathrm{H}$ & -6.29479 & -0.89117 & 0.37661 \\
\hline $\mathrm{H}$ & -5.66886 & -1.09795 & -1.2691 \\
\hline $\mathrm{H}$ & -7.11915 & -1.96526 & -0.75939 \\
\hline $\mathrm{C}$ & -4.89297 & -4.3222 & -1.2444 \\
\hline $\mathrm{H}$ & -4.46939 & -3.86101 & -2.14317 \\
\hline $\mathrm{H}$ & -4.24045 & -5.15464 & -0.95563 \\
\hline $\mathrm{H}$ & -5.86641 & -4.74771 & -1.52104 \\
\hline $\mathrm{C}$ & -3.75989 & 2.99383 & -0.76405 \\
\hline $\mathrm{C}$ & -3.34727 & 4.01666 & -1.62985 \\
\hline $\mathrm{C}$ & -4.40037 & 3.34655 & 0.43047 \\
\hline C & -3.55536 & 5.35638 & -1.30438 \\
\hline $\mathrm{H}$ & -2.86051 & 3.75593 & -2.56706 \\
\hline $\mathrm{C}$ & -4.60797 & 4.68724 & 0.76385 \\
\hline $\mathrm{H}$ & -4.74986 & 2.5651 & 1.10191 \\
\hline
\end{tabular}


C

$\mathrm{H}$

$\mathrm{H}$

C

H

C

H

$\mathrm{H}$

C

$\mathrm{H}$

$\mathrm{H}$

C

C

H

$\mathrm{H}$

C

C

$\mathrm{H}$

C

$\mathrm{H}$

$\mathrm{H}$

$\mathrm{H}$

O

H

C

H

C

H

H

C

C

H

H

$\begin{array}{ccc}-4.1838 & 5.69501 & -0.10252 \\ -3.23704 & 6.13689 & -1.99086 \\ -5.10606 & 4.94243 & 1.69544 \\ -4.34942 & 6.73859 & 0.15146 \\ 4.00523 & -1.64582 & -0.47279 \\ 4.04265 & -1.6974 & 0.62628 \\ 5.3324 & -1.85657 & -0.99799 \\ -2.19143 & 1.07717 & -0.81516 \\ -3.50896 & 1.5422 & -1.12189 \\ -3.59994 & 1.4077 & -2.20491 \\ -4.26909 & 0.90828 & -0.64731 \\ -1.90496 & 0.78817 & 0.56283 \\ -2.28199 & 1.61826 & 1.18232 \\ -2.64625 & -0.47572 & 1.08029 \\ -3.71955 & -0.2712 & 1.00424 \\ -2.4496 & -0.56555 & 2.15475 \\ 1.81395 & 4.09185 & -0.78412 \\ 2.43202 & 4.96277 & -1.04029 \\ 1.52349 & 4.19329 & 0.27105 \\ -0.04561 & -2.70624 & 0.79284 \\ 1.25395 & -2.65872 & 0.56893 \\ -1.70584 & -3.53586 & 1.69629 \\ 1.86506 & -2.10098 & 1.27748 \\ 1.94317 & -3.20701 & -0.61452 \\ 3.13383 & -2.73781 & -1.04275 \\ 3.55159 & -3.16481 & -1.95296 \\ 1.21745 & -4.27964 & -1.39165 \\ 1.02522 & -5.1571 & -0.7616 \\ 0.23957 & -3.92224 & -1.73771 \\ 1.79464 & -4.59976 & -2.26379 \\ 0.66216 & 4.04018 & -1.63212 \\ 0.20719 & 4.89154 & -1.5503 \\ -0.38528 & 0.71696 & 0.70786 \\ -0.1579 & -0.20488 & 1.24597 \\ 0.24714 & 1.98957 & 1.34968 \\ -0.46648 & 2.76072 & 1.66062 \\ 0.89201 & 1.77129 & 2.20601 \\ 2.60138 & 2.80238 & -0.9938 \\ 3.14668 & 2.64384 & -2.44929 \\ 3.06006 & 3.57082 & -3.02438 \\ 2.70566 & 1.84207 & -3.04795\end{array}$




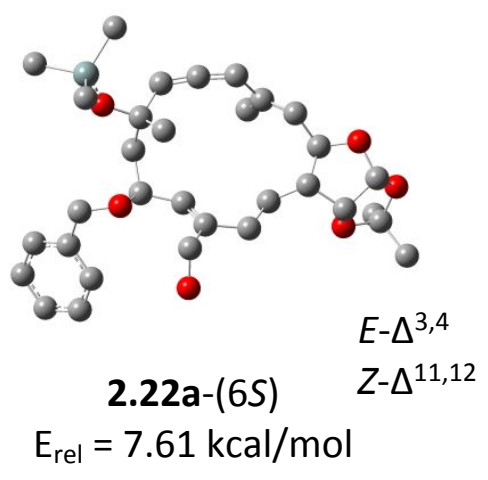

C

C

C

C

$\mathrm{H}$

$\mathrm{H}$

C

$\mathrm{H}$

O

O

C

C

$\mathrm{H}$

$\mathrm{H}$

$\mathrm{H}$

C

$\mathrm{H}$

$\mathrm{H}$

$\mathrm{H}$

C

$\mathrm{H}$

$\mathrm{H}$

C

$\mathrm{H}$

$\mathrm{H}$

C

C

$\mathrm{H}$

C

$\mathrm{H}$

$\mathrm{H}$

$\mathrm{H}$

O

$\mathrm{Si}$

C

$\mathrm{H}$

$\mathrm{H}$

$\mathrm{H}$

$\begin{array}{lcc}1.53669 & -2.48201 & -1.28903 \\ 2.20155 & -1.47578 & -0.32728 \\ -5.13205 & 0.43823 & 1.0306 \\ -6.13187 & -0.71386 & 0.73199 \\ -5.247 & 0.8653 & 2.03525 \\ -6.67898 & -1.11255 & 1.59097 \\ -3.76577 & -0.20506 & 0.78475 \\ -3.50493 & -0.76055 & 1.69767 \\ -5.41934 & 1.41046 & 0.03085 \\ -7.0557 & -0.16191 & -0.18731 \\ -6.76512 & 1.22238 & -0.4075 \\ -7.72494 & 2.08642 & 0.41626 \\ -7.50999 & 3.14891 & 0.26346 \\ -8.7603 & 1.88834 & 0.12203 \\ -7.62337 & 1.86132 & 1.48299 \\ -6.83477 & 1.51751 & -1.89871 \\ -7.84893 & 1.34321 & -2.27013 \\ -6.56276 & 2.55966 & -2.09245 \\ -6.14457 & 0.86233 & -2.43664 \\ -2.58229 & 0.68193 & 0.38515 \\ -1.87614 & 0.00901 & -0.10707 \\ -2.90418 & 1.41083 & -0.36634 \\ -1.84896 & 1.37282 & 1.55118 \\ -2.29472 & 2.35879 & 1.75219 \\ -2.02414 & 0.79616 & 2.47325 \\ -0.33056 & 1.52839 & 1.4329 \\ 0.31782 & 1.25261 & 0.29071 \\ -0.29623 & 0.99662 & -0.56967 \\ 1.60402 & -1.58301 & 1.07993 \\ 1.72301 & -2.60331 & 1.45859 \\ 2.10204 & -0.88299 & 1.75182 \\ 0.53718 & -1.35316 & 1.07173 \\ 3.6136 & -1.74791 & -0.2957 \\ 4.52754 & -2.98939 & 0.37498 \\ 3.76318 & -4.69509 & 0.09578 \\ 4.42523 & -5.46987 & 0.50394 \\ 2.78817 & -4.80391 & 0.58311 \\ 3.6236 & -4.90618 & -0.97031\end{array}$




\begin{tabular}{|c|c|c|c|}
\hline $\mathrm{C}$ & 6.17794 & -2.8684 & -0.52756 \\
\hline $\mathrm{H}$ & 6.05554 & -3.02694 & -1.60515 \\
\hline $\mathrm{H}$ & 6.63346 & -1.88127 & -0.38664 \\
\hline $\mathrm{H}$ & 6.88808 & -3.61787 & -0.15679 \\
\hline $\mathrm{C}$ & 4.81059 & -2.71796 & 2.22404 \\
\hline $\mathrm{H}$ & 5.23658 & -1.72617 & 2.41689 \\
\hline $\mathrm{H}$ & 3.8864 & -2.80042 & 2.80603 \\
\hline $\mathrm{H}$ & 5.51619 & -3.46201 & 2.61583 \\
\hline $\mathrm{C}$ & 4.32328 & 2.85776 & -0.05099 \\
\hline $\mathrm{C}$ & 3.73791 & 4.06462 & 0.35685 \\
\hline C & 5.21633 & 2.87218 & -1.12789 \\
\hline $\mathrm{C}$ & 4.04251 & 5.25737 & -0.29719 \\
\hline $\mathrm{H}$ & 3.03495 & 4.05963 & 1.18564 \\
\hline $\mathrm{C}$ & 5.53143 & 4.06719 & -1.77902 \\
\hline $\mathrm{H}$ & 5.67136 & 1.94053 & -1.45817 \\
\hline C & 4.94375 & 5.26282 & -1.36556 \\
\hline $\mathrm{H}$ & 3.5791 & 6.18543 & 0.02781 \\
\hline $\mathrm{H}$ & 6.22876 & 4.0612 & -2.6128 \\
\hline $\mathrm{H}$ & 5.18276 & 6.19351 & -1.87336 \\
\hline C & -4.13021 & -1.27771 & -0.28334 \\
\hline $\mathrm{H}$ & -4.27034 & -0.78638 & -1.25448 \\
\hline O & -5.39416 & -1.78257 & 0.18477 \\
\hline $\mathrm{C}$ & -3.10382 & -2.36189 & -0.34342 \\
\hline $\mathrm{H}$ & -3.12184 & -3.03892 & 0.51129 \\
\hline $\mathrm{C}$ & -2.0584 & -2.41894 & -1.19415 \\
\hline $\mathrm{C}$ & -1.90247 & -1.56806 & -2.43292 \\
\hline $\mathrm{H}$ & -1.03803 & -0.8984 & -2.35194 \\
\hline $\mathrm{H}$ & -1.71576 & -2.21181 & -3.30106 \\
\hline $\mathrm{H}$ & -2.7834 & -0.95884 & -2.6424 \\
\hline O & 2.64981 & 1.36814 & 1.00291 \\
\hline C & 4.02801 & 1.5657 & 0.68832 \\
\hline $\mathrm{H}$ & 4.52112 & 1.5754 & 1.6677 \\
\hline $\mathrm{H}$ & 4.42359 & 0.70695 & 0.13204 \\
\hline C & 1.77814 & 1.20815 & -0.11218 \\
\hline $\mathrm{H}$ & 1.93946 & 2.05565 & -0.80217 \\
\hline C & 2.07381 & -0.0533 & -0.98053 \\
\hline $\mathrm{H}$ & 3.00382 & 0.10354 & -1.53537 \\
\hline $\mathrm{H}$ & 1.28223 & -0.07672 & -1.73747 \\
\hline $\mathrm{C}$ & 0.30842 & 1.96894 & 2.72916 \\
\hline $\mathrm{H}$ & 1.3782 & 2.13767 & 2.62762 \\
\hline $\mathrm{H}$ & 0.14848 & 1.21561 & 3.5141 \\
\hline $\mathrm{C}$ & 0.33397 & -2.96844 & -1.11901 \\
\hline C & -0.91386 & -3.29902 & -0.85365 \\
\hline $\mathrm{H}$ & -1.12303 & -4.18487 & -0.2519 \\
\hline $\mathrm{H}$ & 2.1227 & -2.72168 & -2.17762 \\
\hline O & -0.31161 & 3.16997 & 3.19606 \\
\hline $\mathrm{H}$ & -0.0412 & 3.33912 & 4.10153 \\
\hline
\end{tabular}




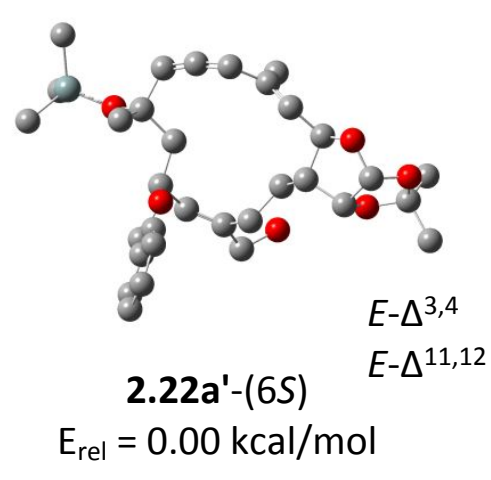

C

C

C

C

$\mathrm{H}$

$\mathrm{H}$

C

$\mathrm{H}$

O

O

C

C

$\mathrm{H}$

$\mathrm{H}$

$\mathrm{H}$

C

$\mathrm{H}$

$\mathrm{H}$

$\mathrm{H}$

C

$\mathrm{H}$

$\mathrm{H}$

C

$\mathrm{H}$

$\mathrm{H}$

C

$\mathrm{H}$

C

$\mathrm{H}$

$\mathrm{H}$

$\mathrm{H}$

0

$\mathrm{Si}$

C

$\mathrm{H}$

$\mathrm{H}$

$\mathrm{H}$

C

$\begin{array}{lcc}1.6321 & -3.26137 & -1.75823 \\ 1.97878 & -2.06605 & -0.84093 \\ -4.28887 & 0.36875 & 0.42655 \\ -5.41567 & -0.57477 & 0.83053 \\ -4.1916 & 1.24454 & 1.03404 \\ -5.62931 & -0.54053 & 1.87812 \\ -3.09929 & -0.52779 & 0.40508 \\ -2.7606 & -0.78785 & 1.38624 \\ -4.65596 & 0.81022 & -0.86119 \\ -6.5183 & -0.14202 & 0.01507 \\ -6.01737 & 1.10948 & -0.47334 \\ -6.0295 & 2.17653 & 0.63622 \\ -5.60669 & 3.08513 & 0.26144 \\ -7.03687 & 2.35426 & 0.94958 \\ -5.45249 & 1.83092 & 1.46891 \\ -6.85436 & 1.62646 & -1.66138 \\ -7.85964 & 1.8024 & -1.3406 \\ -6.43075 & 2.53937 & -2.0252 \\ -6.85287 & 0.89644 & -2.44436 \\ -1.91751 & 0.07127 & -0.40991 \\ -1.15854 & -0.66727 & -0.56655 \\ -2.30859 & 0.39267 & -1.35269 \\ -1.28293 & 1.27096 & 0.31929 \\ -2.04316 & 1.9575 & 0.62429 \\ -0.75721 & 0.91231 & 1.18002 \\ 0.96436 & 1.65502 & -0.90917 \\ 1.55356 & 2.29133 & -1.53548 \\ 1.41455 & -2.34309 & 0.56214 \\ 1.84843 & -3.23927 & 0.95169 \\ 1.64901 & -1.52243 & 1.20702 \\ 0.35211 & -2.45763 & 0.5016 \\ 3.39618 & -1.90134 & -0.77312 \\ 4.27787 & -2.64338 & 0.42497 \\ 4.02579 & -4.56539 & 0.33728 \\ 4.59955 & -5.03584 & 1.10889 \\ 2.98986 & -4.7953 & 0.47245 \\ 4.34952 & -4.92588 & -0.61706 \\ 6.154 & -2.23091 & 0.17988\end{array}$


$\mathrm{H}$

$\mathrm{H}$

$\mathrm{H}$

C

$\mathrm{H}$

$\mathrm{H}$

$\mathrm{H}$

C

$\mathrm{H}$

O

C

$\mathrm{H}$

C

C

$\mathrm{H}$

$\mathrm{H}$

$\mathrm{H}$

C

$\mathrm{H}$

C

$\mathrm{H}$

$\mathrm{H}$

C

C

$\mathrm{H}$

$\mathrm{H}$

C

C

$\mathrm{H}$

$\mathrm{H}$

O

$\mathrm{H}$

O

C

$\mathrm{H}$

$\mathrm{H}$

C

C

C

C

$\mathrm{H}$

C

$\mathrm{H}$

C

$\mathrm{H}$

$\mathrm{H}$

$\mathrm{H}$

\begin{tabular}{|c|c|c|}
\hline 6.47658 & -2.59108 & -0.77495 \\
\hline 6.29386 & -1.172 & 0.22757 \\
\hline 6.72604 & -2.70251 & 0.95039 \\
\hline 3.68524 & -1.9914 & 2.15215 \\
\hline 3.82354 & -0.93149 & 2.1986 \\
\hline 2.64862 & -2.22223 & 2.28288 \\
\hline 4.25556 & -2.46026 & 2.92598 \\
\hline-3.76597 & -1.71823 & -0.28512 \\
\hline-4.00772 & -1.4477 & -1.29114 \\
\hline-4.97062 & -1.93992 & 0.5068 \\
\hline-2.85106 & -2.94993 & -0.283 \\
\hline-3.0492 & -3.78001 & 0.3601 \\
\hline-1.77462 & -2.9388 & -1.07634 \\
\hline-1.44822 & -1.65477 & -1.86338 \\
\hline-0.81997 & -1.0228 & -1.27175 \\
\hline-0.94244 & -1.91172 & -2.77121 \\
\hline-2.35612 & -1.13749 & -2.09643 \\
\hline 1.6090 & 0.36838 & -0.36721 \\
\hline 2.66401 & 0.51411 & -0.27226 \\
\hline 1.33122 & -0.77914 & -1.36498 \\
\hline 1.73596 & -0.53755 & -2.32567 \\
\hline 0.27233 & -0.92101 & -1.44746 \\
\hline 0.42835 & -3.80788 & -1.52632 \\
\hline-0.82815 & -4.13732 & -1.1962 \\
\hline-1.13725 & -5.14603 & -1.0115 \\
\hline 2.29915 & -3.62439 & -2.5141 \\
\hline-0.3231 & 2.01848 & -0.64761 \\
\hline-0.871 & 3.26529 & -1.37164 \\
\hline-1.60746 & 3.74 & -0.75745 \\
\hline-0.0708 & 3.95041 & -1.5606 \\
\hline-1.46553 & 2.8718 & -2.61134 \\
\hline-1.81072 & 3.64424 & -3.06315 \\
\hline 1.0707 & 0.0378 & 0.92203 \\
\hline 1.31665 & 1.11563 & 1.8379 \\
\hline 2.3585 & 1.35535 & 1.83 \\
\hline 0.74998 & 1.97353 & 1.53828 \\
\hline 0.90536 & 0.69564 & 3.26676 \\
\hline 1.56405 & 1.2526 & 4.37429 \\
\hline-0.11952 & -0.2395 & 3.4646 \\
\hline 1.20706 & 0.86424 & 5.67508 \\
\hline 2.34071 & 1.9736 & 4.22712 \\
\hline-0.47292 & -0.63015 & 4.76688 \\
\hline-0.6304 & -0.65705 & 2.6237 \\
\hline 0.19153 & -0.08078 & 5.8711 \\
\hline 1.70979 & 1.28878 & 6.51866 \\
\hline & -1.35032 & 4.9195 \\
\hline & -0 & \\
\hline
\end{tabular}




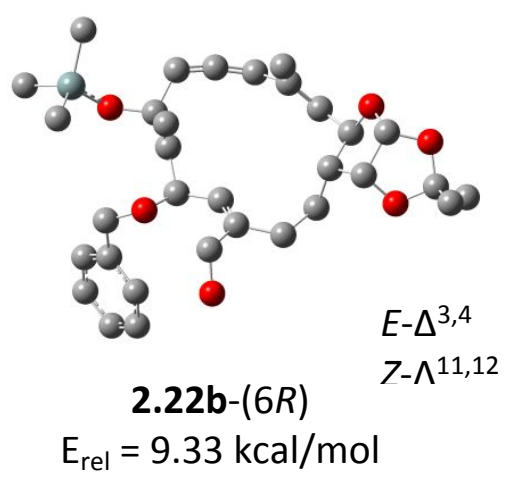

C

C

C

C

$\mathrm{H}$

$\mathrm{H}$

O

O

C

C

$\mathrm{H}$

$\mathrm{H}$

$\mathrm{H}$

C

$\mathrm{H}$

$\mathrm{H}$

$\mathrm{H}$

C

$\mathrm{H}$

$\mathrm{H}$

C

$\mathrm{H}$

$\mathrm{H}$

C

C

$\mathrm{H}$

C

$\mathrm{H}$

$\mathrm{H}$

$\mathrm{H}$

O

$\mathrm{Si}$

C

$\mathrm{H}$

$\mathrm{H}$

$\mathrm{H}$

C

$\mathrm{H}$

$\begin{array}{ccc}1.51839 & -2.80764 & -1.15897 \\ 2.12661 & -1.66247 & -0.31415 \\ -4.6983 & 0.22999 & 0.88676 \\ -5.4789 & -1.03169 & 0.43195 \\ -4.50126 & 0.25094 & 1.9663 \\ -5.57517 & -1.8307 & 1.17307 \\ -5.54426 & 1.31201 & 0.51004 \\ -6.76801 & -0.56185 & 0.08819 \\ -6.88278 & 0.82911 & 0.40396 \\ -7.62249 & 0.99651 & 1.73501 \\ -7.70419 & 2.05645 & 1.99624 \\ -8.6281 & 0.57007 & 1.66602 \\ -7.08672 & 0.48122 & 2.53904 \\ -7.56749 & 1.54836 & -0.74889 \\ -8.58262 & 1.1633 & -0.88341 \\ -7.62219 & 2.62257 & -0.54702 \\ -7.00257 & 1.38664 & -1.67062 \\ -2.78551 & 1.57379 & -0.24863 \\ -2.20042 & 1.52048 & -1.17342 \\ -3.59043 & 2.2891 & -0.4437 \\ -1.91458 & 2.10566 & 0.90748 \\ -1.93907 & 3.20643 & 0.88306 \\ -2.39972 & 1.84676 & 1.86048 \\ -0.43574 & 1.71806 & 1.00659 \\ 0.25695 & 1.25019 & -0.0437 \\ -0.30204 & 1.07766 & -0.96213 \\ 1.42489 & -1.67296 & 1.05562 \\ 1.46687 & -2.67421 & 1.49985 \\ 1.8989 & -0.95619 & 1.72853 \\ 0.37335 & -1.39586 & 0.94443 \\ 3.53359 & -1.90612 & -0.16344 \\ 4.47381 & -2.9139 & 0.79104 \\ 3.90901 & -4.72008 & 0.73848 \\ 4.59207 & -5.33868 & 1.33507 \\ 2.90241 & -4.86278 & 1.14871 \\ 3.91622 & -5.11881 & -0.28289 \\ 6.19223 & -2.7726 & 0.03263 \\ 6.18737 & -3.08966 & -1.01647\end{array}$




\begin{tabular}{|c|c|c|c|}
\hline $\mathrm{H}$ & 6.55838 & -1.73998 & 0.06603 \\
\hline $\mathrm{H}$ & 6.91482 & -3.39933 & 0.56977 \\
\hline $\mathrm{C}$ & 4.52558 & -2.33971 & 2.59173 \\
\hline $\mathrm{H}$ & 4.79357 & -1.27893 & 2.66172 \\
\hline $\mathrm{H}$ & 3.56486 & -2.4733 & 3.10051 \\
\hline $\mathrm{H}$ & 5.27874 & -2.90935 & 3.15139 \\
\hline C & 4.24374 & 2.73927 & -0.07131 \\
\hline $\mathrm{C}$ & 3.62106 & 3.91776 & 0.3628 \\
\hline C & 5.19091 & 2.81596 & -1.09819 \\
\hline $\mathrm{C}$ & 3.94404 & 5.1445 & -0.21523 \\
\hline $\mathrm{H}$ & 2.87489 & 3.86336 & 1.15077 \\
\hline $\mathrm{C}$ & 5.52348 & 4.04478 & -1.67305 \\
\hline $\mathrm{H}$ & 5.67388 & 1.90648 & -1.44987 \\
\hline C & 4.89964 & 5.21233 & -1.23298 \\
\hline $\mathrm{H}$ & 3.45194 & 6.05046 & 0.12948 \\
\hline $\mathrm{H}$ & 6.26246 & 4.08719 & -2.46904 \\
\hline $\mathrm{H}$ & 5.15251 & 6.16945 & -1.68153 \\
\hline O & 2.54477 & 1.19626 & 0.84963 \\
\hline $\mathrm{C}$ & 3.93143 & 1.40982 & 0.5914 \\
\hline $\mathrm{H}$ & 4.39531 & 1.37092 & 1.58453 \\
\hline $\mathrm{H}$ & 4.34886 & 0.58213 & 0.00472 \\
\hline $\mathrm{C}$ & 1.73459 & 1.01685 & -0.31074 \\
\hline $\mathrm{H}$ & 2.00397 & 1.80675 & -1.03468 \\
\hline C & 2.00626 & -0.30955 & -1.0751 \\
\hline $\mathrm{H}$ & 2.93684 & -0.21339 & -1.64277 \\
\hline $\mathrm{H}$ & 1.21035 & -0.38995 & -1.82057 \\
\hline $\mathrm{C}$ & 0.13262 & 2.01764 & 2.37512 \\
\hline $\mathrm{H}$ & 1.20706 & 1.85728 & 2.42449 \\
\hline $\mathrm{H}$ & -0.35318 & 1.38361 & 3.13102 \\
\hline C & 0.44005 & -2.66058 & -1.88262 \\
\hline $\mathrm{C}$ & -0.69228 & -2.31269 & -2.4594 \\
\hline $\mathrm{H}$ & 1.98768 & -3.78192 & -1.03375 \\
\hline $\mathrm{H}$ & -0.695 & -1.97484 & -3.49689 \\
\hline C & -1.94899 & -2.17087 & -1.68453 \\
\hline $\mathrm{C}$ & -2.12727 & -3.08828 & -0.49974 \\
\hline $\mathrm{H}$ & -2.07704 & -4.13289 & -0.83169 \\
\hline $\mathrm{H}$ & -1.31672 & -2.95551 & 0.22643 \\
\hline $\mathrm{H}$ & -3.08582 & -2.92906 & -0.00993 \\
\hline C & -2.78225 & -1.16773 & -2.02681 \\
\hline $\mathrm{H}$ & -2.53309 & -0.59297 & -2.91843 \\
\hline C & -3.42324 & 0.20439 & 0.02958 \\
\hline $\mathrm{H}$ & -2.69431 & -0.43813 & 0.54021 \\
\hline C & -3.9096 & -0.58002 & -1.22067 \\
\hline $\mathrm{H}$ & -4.49406 & 0.09429 & -1.86417 \\
\hline 0 & -4.79452 & -1.57745 & -0.67094 \\
\hline O & -0.15394 & 3.36797 & 2.74846 \\
\hline $\mathrm{H}$ & -0.06107 & 3.46421 & 3.6991 \\
\hline
\end{tabular}




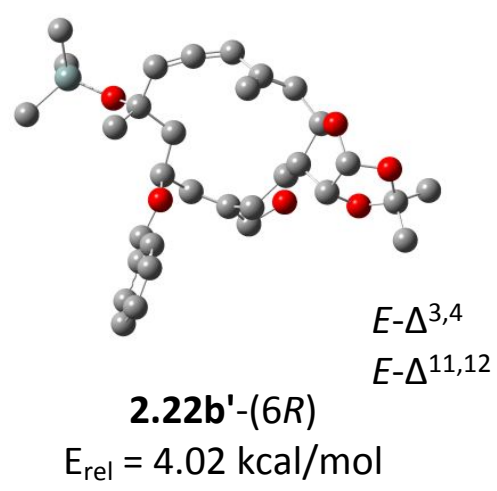

C

C

C

C

$\mathrm{H}$

$\mathrm{H}$

O

O

C

C

$\mathrm{H}$

$\mathrm{H}$

$\mathrm{H}$

C

$\mathrm{H}$

$\mathrm{H}$

$\mathrm{H}$

C

$\mathrm{H}$

$\mathrm{H}$

C

$\mathrm{H}$

$\mathrm{H}$

C

$\mathrm{H}$

C

$\mathrm{H}$

$\mathrm{H}$

$\mathrm{H}$

0

$\mathrm{Si}$

C

$\mathrm{H}$

$\mathrm{H}$

$\mathrm{H}$

C

$\mathrm{H}$

$\mathrm{H}$

$\mathrm{H}$

$$
\begin{array}{ccc}
1.66863 & -3.10639 & -1.15183 \\
1.77293 & -1.86578 & -0.23574 \\
-3.98612 & 0.31492 & 0.22993 \\
-4.37373 & -1.08365 & 0.66958 \\
-3.74417 & 0.98986 & 1.02172 \\
-3.91325 & -1.3596 & 1.58943 \\
-5.17281 & 0.81824 & -0.37504 \\
-5.82069 & -1.04151 & 0.74926 \\
-6.07566 & 0.36624 & 0.67439 \\
-5.77298 & 1.04287 & 2.0215 \\
-5.90788 & 2.09939 & 1.93505 \\
-6.44377 & 0.66112 & 2.76666 \\
-4.76647 & 0.83536 & 2.30555 \\
-7.55225 & 0.66273 & 0.3224 \\
-8.18569 & 0.29528 & 1.10266 \\
-7.69272 & 1.71849 & 0.22087 \\
-7.80377 & 0.18047 & -0.59522 \\
-2.65015 & 1.36564 & -1.62738 \\
-2.11209 & 1.23394 & -2.55179 \\
-3.64904 & 1.67695 & -1.81487 \\
-1.86919 & 2.43203 & -0.8019 \\
-2.23843 & 3.39514 & -1.02649 \\
-2.00877 & 2.20665 & 0.24463 \\
0.79471 & 1.85335 & -0.99231 \\
1.63684 & 2.23771 & -1.5302 \\
1.08278 & -2.12078 & 1.11285 \\
1.5585 & -2.9422 & 1.60684 \\
1.15835 & -1.24789 & 1.72317 \\
0.05312 & -2.3547 & 0.94856 \\
3.16535 & -1.59267 & -0.03411 \\
4.08622 & -2.42231 & 1.08441 \\
3.91431 & -4.34044 & 0.79915 \\
4.52238 & -4.86319 & 1.50626 \\
2.89195 & -4.63129 & 0.92604 \\
4.23086 & -4.58659 & -0.19275 \\
5.94169 & -1.89268 & 0.83363 \\
6.24453 & -2.13244 & -0.16604 \\
6.03684 & -0.83945 & 0.98824 \\
6.56191 & -2.4129 & 1.52779
\end{array}
$$




\begin{tabular}{|c|c|c|c|}
\hline $\mathrm{C}$ & 3.49393 & -2.00652 & 2.89205 \\
\hline $\mathrm{H}$ & 3.58793 & -0.95586 & 3.06921 \\
\hline $\mathrm{H}$ & 2.46959 & -2.2941 & 3.00687 \\
\hline $\mathrm{H}$ & 4.09349 & -2.54166 & 3.59785 \\
\hline C & 1.1539 & 0.63794 & -0.10776 \\
\hline $\mathrm{H}$ & 2.15883 & 0.78754 & 0.22885 \\
\hline C & 1.09485 & -0.65828 & -0.96044 \\
\hline $\mathrm{H}$ & 1.626 & -0.49585 & -1.87284 \\
\hline $\mathrm{H}$ & 0.05991 & -0.88501 & -1.17014 \\
\hline C & 0.59837 & -3.15834 & -1.95585 \\
\hline $\mathrm{C}$ & -0.54345 & -3.06816 & -2.6443 \\
\hline $\mathrm{H}$ & 2.41547 & -3.86916 & -1.15204 \\
\hline $\mathrm{H}$ & -0.67188 & -3.47693 & -3.62479 \\
\hline C & -1.6273 & -2.29853 & -1.91784 \\
\hline C & -1.50899 & -2.1115 & -0.3872 \\
\hline $\mathrm{H}$ & -1.01679 & -2.95617 & 0.04365 \\
\hline $\mathrm{H}$ & -0.93983 & -1.22602 & -0.18428 \\
\hline $\mathrm{H}$ & -2.48445 & -2.01084 & 0.044 \\
\hline C & -2.68585 & -1.73862 & -2.5035 \\
\hline $\mathrm{H}$ & -2.83134 & -1.70055 & -3.56172 \\
\hline C & -2.89224 & 0.07134 & -0.7985 \\
\hline $\mathrm{H}$ & -1.94728 & -0.27136 & -0.38182 \\
\hline C & -3.60121 & -1.10721 & -1.49869 \\
\hline $\mathrm{H}$ & -4.52262 & -0.81048 & -1.97233 \\
\hline O & -3.89484 & -1.9916 & -0.37377 \\
\hline $\mathrm{C}$ & -0.36836 & 2.5336 & -1.23771 \\
\hline $\mathrm{C}$ & -0.16189 & 3.75871 & -2.16591 \\
\hline $\mathrm{H}$ & -0.86081 & 4.51997 & -1.8769 \\
\hline $\mathrm{H}$ & 0.84542 & 4.13275 & -2.07128 \\
\hline O & -0.39024 & 3.40395 & -3.5414 \\
\hline $\mathrm{H}$ & -0.27254 & 4.17654 & -4.09983 \\
\hline O & 0.29002 & 0.52003 & 1.02569 \\
\hline $\mathrm{C}$ & 0.75749 & 1.37407 & 2.07306 \\
\hline $\mathrm{H}$ & 0.14289 & 2.24863 & 2.12121 \\
\hline $\mathrm{H}$ & 1.76951 & 1.65979 & 1.87536 \\
\hline C & 0.68894 & 0.62389 & 3.41624 \\
\hline C & 1.76481 & 0.68077 & 4.30265 \\
\hline $\mathrm{C}$ & -0.44874 & -0.11234 & 3.74669 \\
\hline C & 1.70321 & 0.00099 & 5.51893 \\
\hline $\mathrm{H}$ & 2.6619 & 1.26066 & 4.0415 \\
\hline C & -0.51092 & -0.79157 & 4.96373 \\
\hline $\mathrm{H}$ & -1.29686 & -0.15713 & 3.04826 \\
\hline $\mathrm{C}$ & 0.56485 & -0.73514 & 5.84978 \\
\hline $\mathrm{H}$ & 2.55143 & 0.04527 & 6.21739 \\
\hline $\mathrm{H}$ & -1.40823 & -1.37164 & 5.22419 \\
\hline $\mathrm{H}$ & 0.51648 & -1.27099 & 6.80885 \\
\hline
\end{tabular}




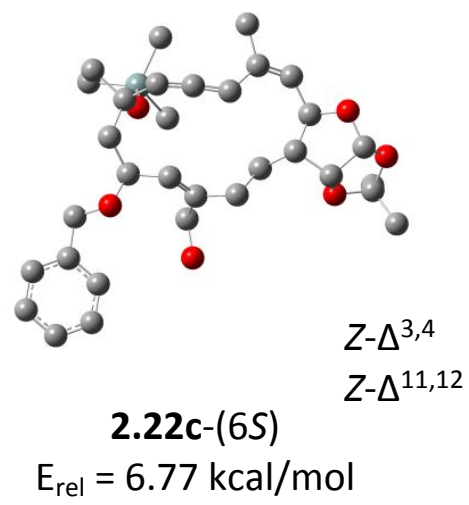

$-0.29493$

0.94579

1. 30173

$-1.50035$

$-1.54549$

$-2.77464$

$-3.87804$

$-4.75472$

$-4.17426$

$-4.3646$

$-3.22915$

$-2.93771$

$-5.40489$

$-4.19643$

$-3.91451$

$-5.58128$

$-6.04798$

$-1.98641$

$-1.6456$

$-2.278$

$-4.33129$

$-6.40962$

$-5.72002$

$-0.80073$

$-0.94203$

$-0.79825$

0.53243

1. 37602

1.11237

2.68133

2.68712

3.00373

3.99728

3.09943

2.0282

1.45793

1.04466

$-0.05653$
$2.13042-1.83268$

$2.52356-1.94317$

$2.84285-2.92602$

$1.60267-1.77795$

$0.53639-1.97275$

$2.28803-1.49191$

$1.68005-1.00812$

$2.3131-0.87244$

$0.27863-0.53515$

$\begin{array}{lll}0.33649 & 0.55112\end{array}$

$-0.91429-0.78793$

$-0.88622-1.84576$

$-0.12499-1.18708$

$-2.10477-0.64768$

$-2.96144-1.27253$

$-1.50361-1.00981$

$-1.88588-1.92256$

$\begin{array}{ll}-0.96009 & 0.11656\end{array}$

$0.06554 \quad 0.28996$

$-1.36232 \quad 1.09112$

$\begin{array}{ll}-2.50269 & 0.71232\end{array}$

$\begin{array}{ll}-1.8057 & 0.10143\end{array}$

$\begin{array}{ll}-2.67794 & 0.99779\end{array}$

$\begin{array}{ll}-1.76986 & -0.47248\end{array}$

$\begin{array}{ll}-2.8381 & -0.25329\end{array}$

$-1.67478-1.56579$

$\begin{array}{ll}-1.28523 & 0.06408\end{array}$

$-0.61606-0.73837$

$-0.51101-1.7898$

$0.01778-0.33625$

$0.21163 \quad 0.74234$

$1.34174-1.06342$

$1.6577-0.72133$

$1.13303-2.13605$

$2.54461-0.85448$

$2.42447 \quad 0.43983$

$3.45272 \quad 1.69734$

$4.89183 \quad 1.16211$ 


\begin{tabular}{|c|c|c|c|}
\hline $\mathrm{H}$ & -0.29903 & 5.51586 & 2.03212 \\
\hline $\mathrm{H}$ & 0.41647 & 5.5417 & 0.41718 \\
\hline $\mathrm{H}$ & -1.00117 & 4.53043 & 0.74192 \\
\hline C & 2.58224 & 4.13399 & 2.5637 \\
\hline $\mathrm{H}$ & 2.29722 & 4.64578 & 3.49202 \\
\hline $\mathrm{H}$ & 3.27806 & 3.33019 & 2.83234 \\
\hline $\mathrm{H}$ & 3.12702 & 4.8575 & 1.94716 \\
\hline C & 0.0957 & 2.34283 & 2.88528 \\
\hline $\mathrm{H}$ & -0.80396 & 1.93838 & 2.40779 \\
\hline $\mathrm{H}$ & 0.70935 & 1.49523 & 3.21097 \\
\hline $\mathrm{H}$ & -0.21722 & 2.89335 & 3.7811 \\
\hline C & 2.82924 & 3.85116 & -1.01801 \\
\hline $\mathrm{H}$ & 2.17979 & 4.72832 & -0.93229 \\
\hline $\mathrm{H}$ & 3.61661 & 3.91516 & -0.26047 \\
\hline $\mathrm{H}$ & 3.30706 & 3.8841 & -2.00373 \\
\hline O & 3.72358 & -0.93496 & -0.63859 \\
\hline C & 4.91214 & -0.80022 & 0.11716 \\
\hline $\mathrm{H}$ & 5.49734 & 0.08139 & -0.19199 \\
\hline $\mathrm{H}$ & 4.65942 & -0.64959 & 1.18296 \\
\hline $\mathrm{C}$ & 5.76156 & -2.04421 & -0.03604 \\
\hline C & 7.14738 & -1.97045 & 0.14601 \\
\hline $\mathrm{C}$ & 5.17936 & -3.28517 & -0.31692 \\
\hline C & 7.93894 & -3.11633 & 0.06147 \\
\hline $\mathrm{H}$ & 7.61227 & -1.00814 & 0.35268 \\
\hline C & 5.97115 & -4.43057 & -0.41012 \\
\hline $\mathrm{H}$ & 4.10746 & -3.33659 & -0.47592 \\
\hline C & 7.35188 & -4.35178 & -0.21798 \\
\hline $\mathrm{H}$ & 9.01401 & -3.04225 & 0.20441 \\
\hline $\mathrm{H}$ & 5.50768 & -5.38797 & -0.63502 \\
\hline $\mathrm{H}$ & 7.96681 & -5.245 & -0.2913 \\
\hline $\mathrm{C}$ & -2.80524 & 3.77045 & -1.79325 \\
\hline $\mathrm{H}$ & -1.99294 & 4.29387 & -1.2752 \\
\hline $\mathrm{H}$ & -2.65692 & 3.95026 & -2.86572 \\
\hline $\mathrm{H}$ & -3.75415 & 4.22446 & -1.49406 \\
\hline C & 0.77346 & -1.56381 & 1.5279 \\
\hline $\mathrm{H}$ & 1.75432 & -1.23129 & 1.87365 \\
\hline $\mathrm{H}$ & 0.01217 & -1.08254 & 2.15571 \\
\hline C & -6.14043 & -4.12914 & 0.74181 \\
\hline $\mathrm{H}$ & -5.60469 & -4.80714 & 1.41396 \\
\hline $\mathrm{H}$ & -7.21638 & -4.24625 & 0.90442 \\
\hline $\mathrm{H}$ & -5.91738 & -4.41749 & -0.29078 \\
\hline C & -5.98002 & -2.23032 & 2.42831 \\
\hline $\mathrm{H}$ & -7.04584 & -2.31243 & 2.66108 \\
\hline $\mathrm{H}$ & -5.41748 & -2.85474 & 3.12916 \\
\hline $\mathrm{H}$ & -5.67011 & -1.18909 & 2.54946 \\
\hline O & 0.67978 & -2.96835 & 1.77965 \\
\hline & 0.90905 & -3.14587 & 2.69481 \\
\hline
\end{tabular}




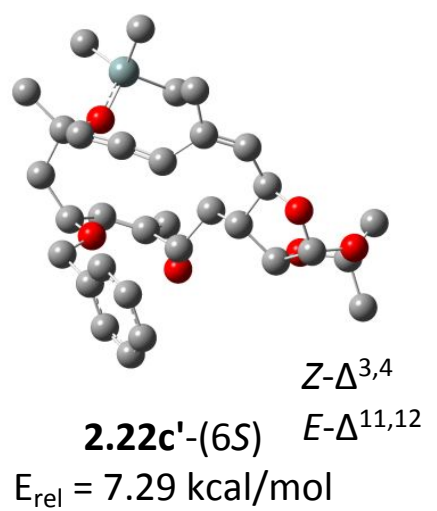

C

C

$\mathrm{H}$

C

$\mathrm{H}$

C

C

$\mathrm{H}$

C

$\mathrm{H}$

C

$\mathrm{H}$

O

C

$\mathrm{H}$

C

$\mathrm{H}$

C

$\mathrm{H}$

$\mathrm{H}$

0

0

C

C

$\mathrm{H}$

$\mathrm{H}$

C

$\mathrm{H}$

C

$\mathrm{H}$

C

$\mathrm{H}$

$\mathrm{H}$

C

O

$\mathrm{Si}$

C

$\mathrm{H}$

$\begin{array}{ccc}-0.31927 & 2.20744 & -1.77665 \\ 0.88927 & 2.78317 & -1.9414 \\ 1.06397 & 3.32912 & -2.83983 \\ -1.53324 & 1.62925 & -1.61121 \\ -1.60624 & 0.58716 & -1.71942 \\ -2.82265 & 2.43674 & -1.30559 \\ -3.97641 & 1.82382 & -0.88109 \\ -4.84089 & 2.45768 & -0.82006 \\ -4.23047 & 0.31972 & -0.48996 \\ -4.31542 & 0.23082 & 0.58212 \\ -3.17422 & -0.75755 & -0.92489 \\ -2.91617 & -0.66922 & -1.96131 \\ -5.42897 & -0.05054 & -1.17746 \\ -4.09984 & -2.00207 & -0.65834 \\ -3.82772 & -2.94036 & -1.09606 \\ -5.50768 & -1.46809 & -1.05811 \\ -5.8451 & -1.86131 & -2.00051 \\ -1.83459 & -0.60489 & 0.02022 \\ -1.60239 & 0.42668 & -0.00948 \\ -2.16069 & -0.9178 & 0.99887 \\ -4.21333 & -2.2045 & 0.72256 \\ -6.36131 & -1.77647 & 0.06491 \\ -5.59444 & -2.649 & 0.8906 \\ -0.43127 & -1.36054 & -0.25649 \\ -0.54679 & -2.4077 & -0.11835 \\ -0.04966 & -1.16933 & -1.23548 \\ 1.66699 & 0.13637 & 0.63107 \\ 1.92694 & 0.81653 & 1.40646 \\ 2.49002 & 0.1504 & -0.67175 \\ 3.35981 & -0.46573 & -0.55735 \\ 3.04318 & 1.61493 & -1.08014 \\ 3.85863 & 1.83745 & -0.42568 \\ 3.38668 & 1.617 & -2.10387 \\ 2.00553 & 2.76139 & -0.86454 \\ 1.45356 & 2.50665 & 0.44968 \\ 1.0433 & 3.43559 & 1.8022 \\ -0.22265 & 4.84789 & 1.38974 \\ -0.47196 & 5.37366 & 2.29497\end{array}$


H

$\mathrm{H}$

C

$\mathrm{H}$

$\mathrm{H}$

$\mathrm{H}$

C

$\mathrm{H}$

$\mathrm{H}$

$\mathrm{H}$

C

$\mathrm{H}$

$\mathrm{H}$

$\mathrm{H}$

C

$\mathrm{H}$

$\mathrm{H}$

$\mathrm{H}$

C

$\mathrm{H}$

$\mathrm{H}$

$\mathrm{H}$

C

$\mathrm{H}$

$\mathrm{H}$

$\mathrm{H}$

C

O

C

$\mathrm{H}$

$\mathrm{H}$

C

C

C

C

$\mathrm{H}$

C

$\mathrm{H}$

C

$\mathrm{H}$

$\mathrm{H}$

$\mathrm{H}$

C

$\mathrm{H}$

$\mathrm{H}$

O

$\mathrm{H}$

\begin{tabular}{|c|c|c|}
\hline 0.20876 & 5.53277 & 0.69236 \\
\hline-1.11181 & 4.42405 & 0.97309 \\
\hline 2.61065 & 4.24994 & 2.62055 \\
\hline 2.31842 & 4.74252 & 3.53153 \\
\hline 3.33357 & 3.49281 & 2.84775 \\
\hline 3.04107 & 4.96623 & 1.9552 \\
\hline 0.24102 & 2.18541 & 3.03685 \\
\hline-0.62375 & 1.74509 & 2.582 \\
\hline 0.94778 & 1.4159 & 3.27586 \\
\hline-0.04685 & 2.69364 & 3.932 \\
\hline 2.75501 & 4.12752 & -0.940 \\
\hline 2.07229 & 4.92553 & -0.73 \\
\hline 3.54811 & 4.13927 & -0.2244 \\
\hline 3.16 & 4.25517 & -1.92 \\
\hline-2.8845 & 3.97797 & $-1.4 \xi$ \\
\hline-1.98549 & 4.42856 & -1.10 \\
\hline-2.99174 & 4.20547 & -2.5 \\
\hline-3.7238 & 4.37465 & -0.9 \\
\hline-5.93463 & -4.13079 & 0.51 \\
\hline-5.35532 & -4.79893 & 1.12359 \\
\hline-6.98 & -4.3006 & 0.71 \\
\hline-5.7 & -4.31897 & -0.51 \\
\hline-5.891 & -2.4221 & 2.36 \\
\hline-6.91 & -2.60074 & 2.5712 \\
\hline-5.28572 & -3.07999 & 2.95 \\
\hline-5.64732 & -1.40205 & 2.6091 \\
\hline 0.59449 & -0.79366 & 0.82995 \\
\hline 1.67368 & -0.46469 & -1.58764 \\
\hline 2.5614 & -1.34731 & -2.26214 \\
\hline 2.91072 & -2.13742 & -1.60348 \\
\hline 3.40757 & -0.80569 & -2.61412 \\
\hline 1.78243 & -1.92983 & -3.401 \\
\hline 1.21092 & -3.20659 & -3.34863 \\
\hline 1.63817 & -1.11756 & -4.50431 \\
\hline 0.4 & -3.66074 & -4.449 \\
\hline 1.32954 & -3.82809 & -2.48107 \\
\hline 0.89699 & -1.55253 & -5.58914 \\
\hline 2.09319 & -0.14835 & -4.50442 \\
\hline 0.32077 & -2.82851 & -5.56895 \\
\hline 0.04652 & -4.63675 & -4.43962 \\
\hline 0.76782 & -0.91167 & -6.43191 \\
\hline-0.24263 & -3.16706 & -6.40858 \\
\hline 0.38628 & -1.3269 & 2.2596 \\
\hline-0.41277 & -0.79065 & 2.72744 \\
\hline 1.28488 & -1.19265 & 2.82475 \\
\hline 0.05988 & -2.71814 & 2.20661 \\
\hline 1346 & -3.15146 & 3.00379 \\
\hline
\end{tabular}




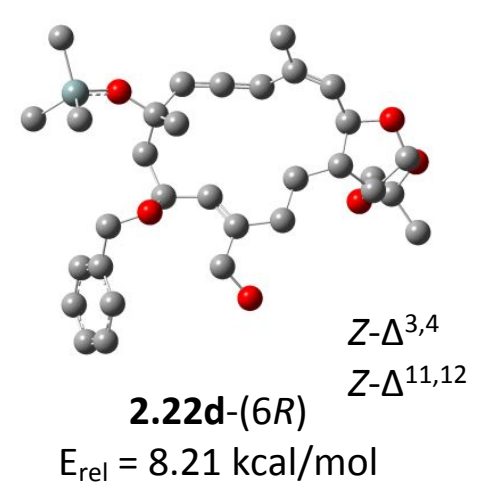

\begin{tabular}{|c|c|c|c|}
\hline $\mathrm{C}$ & -0.98619 & -2.7249 & 1.23076 \\
\hline $\mathrm{C}$ & -2.04172 & -1.90585 & 0.45329 \\
\hline $\mathrm{C}$ & 4.94042 & 0.71115 & -0.88211 \\
\hline C & 6.15134 & -0.22396 & -0.61781 \\
\hline $\mathrm{H}$ & 5.0809 & 1.36919 & -1.74917 \\
\hline $\mathrm{H}$ & 6.90125 & -0.27664 & -1.41246 \\
\hline C & 3.75789 & -0.25268 & -1.02929 \\
\hline $\mathrm{H}$ & 3.73321 & -0.58971 & -2.0754 \\
\hline O & 4.8262 & 1.47316 & 0.31588 \\
\hline O & 6.75744 & 0.28794 & 0.55465 \\
\hline C & 6.09834 & 1.49024 & 0.96376 \\
\hline C & 6.91524 & 2.70509 & 0.51247 \\
\hline $\mathrm{H}$ & 6.41509 & 3.63321 & 0.80732 \\
\hline $\mathrm{H}$ & 7.91187 & 2.68065 & 0.96411 \\
\hline $\mathrm{H}$ & 7.03286 & 2.70446 & -0.57615 \\
\hline C & 5.87618 & 1.45244 & 2.46857 \\
\hline $\mathrm{H}$ & 6.83726 & 1.42323 & 2.99055 \\
\hline $\mathrm{H}$ & 5.32592 & 2.34096 & 2.79327 \\
\hline $\mathrm{H}$ & 5.303 & 0.56024 & 2.73385 \\
\hline C & 2.3757 & 0.23855 & -0.60537 \\
\hline $\mathrm{H}$ & 1.73709 & -0.64629 & -0.5873 \\
\hline $\mathrm{H}$ & 2.4374 & 0.60758 & 0.42479 \\
\hline $\mathrm{C}$ & 1.68817 & 1.28354 & -1.48558 \\
\hline $\mathrm{H}$ & 2.19602 & 2.25616 & -1.39646 \\
\hline $\mathrm{H}$ & 1.79462 & 0.99897 & -2.54494 \\
\hline $\mathrm{C}$ & 0.19693 & 1.47579 & -1.21219 \\
\hline C & -0.41158 & 0.83467 & -0.19984 \\
\hline $\mathrm{H}$ & 0.21148 & 0.21944 & 0.44235 \\
\hline C & -1.71446 & -1.91631 & -1.04667 \\
\hline $\mathrm{H}$ & -1.78068 & -2.94547 & -1.41352 \\
\hline $\mathrm{H}$ & -2.4 & -1.28047 & -1.6082 \\
\hline $\mathrm{H}$ & -0.70039 & -1.55103 & -1.22312 \\
\hline O & -3.24863 & -2.64233 & 0.69227 \\
\hline $\mathrm{Si}$ & -4.8322 & -2.71881 & 0.16889 \\
\hline $\mathrm{C}$ & -5.28774 & -4.54481 & 0.26134 \\
\hline $\mathrm{H}$ & -6.34121 & -4.70709 & 0.00114 \\
\hline $\mathrm{H}$ & -4.67671 & -5.14023 & -0.42693 \\
\hline $\mathrm{H}$ & -5.12864 & -4.93992 & 1.27136 \\
\hline
\end{tabular}




\begin{tabular}{|c|c|c|c|}
\hline $\mathrm{C}$ & -5.94501 & -1.75374 & 1.35628 \\
\hline $\mathrm{H}$ & -5.77284 & -2.06329 & 2.39389 \\
\hline $\mathrm{H}$ & -5.78439 & -0.67055 & 1.30194 \\
\hline $\mathrm{H}$ & -7.00278 & -1.9373 & 1.12769 \\
\hline $\mathrm{C}$ & -5.10455 & -2.08277 & -1.59023 \\
\hline $\mathrm{H}$ & -4.80114 & -1.03697 & -1.70802 \\
\hline $\mathrm{H}$ & -4.55307 & -2.67421 & -2.32965 \\
\hline $\mathrm{H}$ & -6.17107 & -2.14958 & -1.84232 \\
\hline $\mathrm{C}$ & -3.95158 & 3.0859 & -0.03327 \\
\hline $\mathrm{C}$ & -3.7963 & 4.06421 & -1.02602 \\
\hline $\mathrm{C}$ & -4.0384 & 3.49528 & 1.30293 \\
\hline C & -3.71709 & 5.4147 & -0.69054 \\
\hline $\mathrm{H}$ & -3.74234 & 3.76169 & -2.06927 \\
\hline C & -3.95945 & 4.84744 & 1.64456 \\
\hline $\mathrm{H}$ & -4.17802 & 2.74973 & 2.083 \\
\hline $\mathrm{C}$ & -3.79646 & 5.80933 & 0.64786 \\
\hline $\mathrm{H}$ & -3.60022 & 6.16083 & -1.47208 \\
\hline $\mathrm{H}$ & -4.02841 & 5.14736 & 2.68686 \\
\hline $\mathrm{H}$ & -3.73746 & 6.86232 & 0.91005 \\
\hline $\mathrm{C}$ & 4.23764 & -1.45565 & -0.16663 \\
\hline $\mathrm{H}$ & 4.08903 & -1.20278 & 0.89403 \\
\hline O & 5.64871 & -1.52594 & -0.44292 \\
\hline 0 & -2.77909 & 1.03668 & -0.79313 \\
\hline C & -4.02898 & 1.61915 & -0.40784 \\
\hline $\mathrm{H}$ & -4.66944 & 1.4888 & -1.28612 \\
\hline $\mathrm{H}$ & -4.48299 & 1.05226 & 0.41696 \\
\hline C & -1.84896 & 0.81757 & 0.2794 \\
\hline $\mathrm{H}$ & -1.96312 & 1.64441 & 1.0008 \\
\hline C & -2.1882 & -0.46631 & 1.08793 \\
\hline $\mathrm{H}$ & -3.2262 & -0.37989 & 1.42201 \\
\hline $\mathrm{H}$ & -1.58689 & -0.42772 & 2.00386 \\
\hline C & -0.46818 & 2.4166 & -2.18644 \\
\hline $\mathrm{H}$ & -1.53131 & 2.52983 & -1.98994 \\
\hline $\mathrm{H}$ & -0.34122 & 2.05756 & -3.21772 \\
\hline $\mathrm{C}$ & 0.31123 & -2.6781 & 1.08028 \\
\hline C & 1.61662 & -2.56122 & 0.93207 \\
\hline $\mathrm{H}$ & -1.42377 & -3.40229 & 1.96468 \\
\hline $\mathrm{H}$ & 2.12341 & -1.81872 & 1.54994 \\
\hline $\mathrm{C}$ & 2.44675 & -3.27993 & -0.05668 \\
\hline C & 3.6154 & -2.77819 & -0.50854 \\
\hline $\mathrm{H}$ & 4.17079 & -3.34023 & -1.25764 \\
\hline $\mathrm{C}$ & 1.90269 & -4.58366 & -0.58855 \\
\hline $\mathrm{H}$ & 1.7791 & -5.31509 & 0.21992 \\
\hline $\mathrm{H}$ & 0.91077 & -4.4403 & -1.03481 \\
\hline $\mathrm{H}$ & 2.56369 & -5.0128 & -1.34708 \\
\hline 0 & 0.15302 & 3.70331 & -2.12821 \\
\hline $\mathrm{H}$ & 0.06782 & 4.1391 & -2.97935 \\
\hline
\end{tabular}




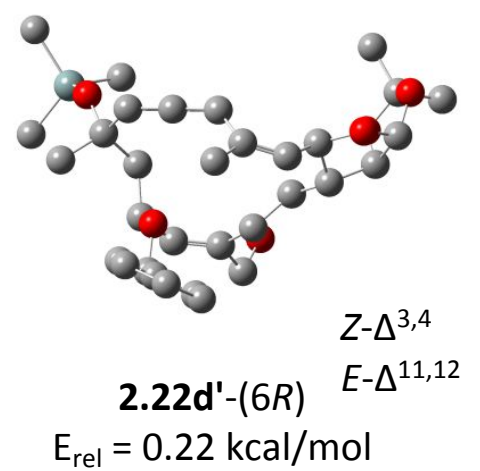

C

C

C

C

$\mathrm{H}$

$\mathrm{H}$

C

$\mathrm{H}$

O

O

C

C

$\mathrm{H}$

$\mathrm{H}$

$\mathrm{H}$

C

$\mathrm{H}$

$\mathrm{H}$

$\mathrm{H}$

C

$\mathrm{H}$

$\mathrm{H}$

C

$\mathrm{H}$

$\mathrm{H}$

C

$\mathrm{H}$

C

$\mathrm{H}$

$\mathrm{H}$

$\mathrm{H}$

O

$\mathrm{Si}$

C

$\mathrm{H}$

$\mathrm{H}$

$\mathrm{H}$

C

$\begin{array}{rrr}-0.97174 & -2.82296 & 1.12661 \\ -2.13815 & -2.00218 & 0.40582 \\ 4.96977 & 0.73098 & -0.66936 \\ 6.22072 & -0.22379 & -0.67289 \\ 5.10634 & 1.63983 & -1.24085 \\ 6.85425 & -0.22649 & -1.53561 \\ 3.77963 & -0.17537 & -1.05085 \\ 3.73358 & -0.33482 & -2.1261 \\ 4.79497 & 1.05113 & 0.66937 \\ 6.91872 & 0.12923 & 0.54931 \\ 6.1409 & 1.17983 & 1.16722 \\ 6.91754 & 2.51761 & 0.85415 \\ 6.41069 & 3.35841 & 1.27158 \\ 7.89922 & 2.44372 & 1.29546 \\ 7.01902 & 2.65763 & -0.20006 \\ 5.99174 & 1.01783 & 2.67849 \\ 6.94365 & 0.96697 & 3.13637 \\ 5.44352 & 1.84492 & 3.08138 \\ 5.44813 & 0.11305 & 2.86289 \\ 2.32407 & 0.30051 & -0.36655 \\ 1.59908 & -0.45025 & -0.63421 \\ 2.55504 & 0.29842 & 0.66287 \\ 1.56588 & 1.7378 & -0.6406 \\ 2.34409 & 2.52291 & -0.74616 \\ 0.99148 & 1.66927 & -1.54024 \\ -0.78567 & 1.5567 & 0.98187 \\ -1.12374 & 1.78456 & 1.97538 \\ -1.87685 & -1.91814 & -1.13981 \\ -1.83357 & -2.90736 & -1.55287 \\ -2.63339 & -1.36344 & -1.61875 \\ -0.91729 & -1.4359 & -1.30061 \\ -3.29568 & -2.70183 & 0.83879 \\ -4.8419 & -2.90649 & 0.26237 \\ -5.25385 & -4.78414 & 0.42011 \\ -6.27805 & -4.93831 & 0.18042 \\ -4.64286 & -5.35763 & -0.24705 \\ -5.06257 & -5.09171 & 1.42368 \\ -5.95115 & -1.87288 & 1.42098\end{array}$




\begin{tabular}{|c|c|c|c|}
\hline $\mathrm{H}$ & -5.74605 & -2.14957 & 2.43117 \\
\hline $\mathrm{H}$ & -5.73369 & -0.83733 & 1.28103 \\
\hline $\mathrm{H}$ & -6.97297 & -2.05972 & 1.19574 \\
\hline $\mathrm{C}$ & -5.17765 & -2.35589 & -1.59506 \\
\hline $\mathrm{H}$ & -4.94396 & -1.32359 & -1.71509 \\
\hline $\mathrm{H}$ & -4.58093 & -2.93303 & -2.27357 \\
\hline $\mathrm{H}$ & -6.21601 & -2.51689 & -1.82288 \\
\hline $\mathrm{C}$ & 4.28712 & -1.47313 & -0.28747 \\
\hline $\mathrm{H}$ & 4.13575 & -1.31773 & 0.76846 \\
\hline O & 5.67486 & -1.5685 & -0.5971 \\
\hline $\mathrm{C}$ & -1.72196 & 0.60618 & 0.17221 \\
\hline $\mathrm{H}$ & -2.60944 & 1.10956 & -0.17096 \\
\hline $\mathrm{C}$ & -2.21452 & -0.58431 & 1.08992 \\
\hline $\mathrm{H}$ & -3.21764 & -0.27014 & 1.35281 \\
\hline $\mathrm{H}$ & -1.63081 & -0.7508 & 1.97677 \\
\hline C & 0.35414 & -2.65716 & 0.91724 \\
\hline C & 1.66861 & -2.49263 & 0.70968 \\
\hline $\mathrm{H}$ & -1.29885 & -3.57048 & 1.80988 \\
\hline $\mathrm{H}$ & 2.16415 & -1.76183 & 1.27557 \\
\hline $\mathrm{C}$ & 2.44953 & -3.29732 & -0.34465 \\
\hline C & 3.62698 & -2.80854 & -0.77296 \\
\hline $\mathrm{H}$ & 4.14024 & -3.37062 & -1.50973 \\
\hline C & 1.87921 & -4.58719 & -0.94998 \\
\hline $\mathrm{H}$ & 1.81985 & -5.35185 & -0.1824 \\
\hline $\mathrm{H}$ & 0.9069 & -4.39626 & -1.3236 \\
\hline $\mathrm{H}$ & 2.51734 & -4.92472 & -1.72567 \\
\hline $\mathrm{C}$ & 0.48447 & 2.15036 & 0.6225 \\
\hline C & 0.68888 & 3.28519 & 1.70226 \\
\hline $\mathrm{H}$ & 1.33582 & 4.11988 & 1.39134 \\
\hline $\mathrm{H}$ & -0.32074 & 3.68096 & 1.8897 \\
\hline O & 1.24736 & 2.65831 & 2.90561 \\
\hline $\mathrm{H}$ & 1.30626 & 3.2728 & 3.64728 \\
\hline O & -1.10102 & 0.19344 & -0.975 \\
\hline $\mathrm{C}$ & -1.62349 & 1.21812 & -1.93269 \\
\hline $\mathrm{H}$ & -1.13008 & 2.18551 & -1.83282 \\
\hline $\mathrm{H}$ & -2.68746 & 1.39603 & -1.78139 \\
\hline $\mathrm{C}$ & -1.40752 & 0.58889 & -3.29839 \\
\hline $\mathrm{C}$ & -2.39276 & -0.27567 & -3.80264 \\
\hline C & -0.24513 & 0.8318 & -4.01279 \\
\hline C & -2.19395 & -0.91697 & -5.02856 \\
\hline $\mathrm{H}$ & -3.29132 & -0.45473 & -3.24672 \\
\hline $\mathrm{C}$ & -0.04504 & 0.19107 & -5.2398 \\
\hline $\mathrm{H}$ & 0.49511 & 1.49682 & -3.62354 \\
\hline C & -1.0172 & -0.68349 & -5.74822 \\
\hline $\mathrm{H}$ & -2.93752 & -1.58606 & -5.41394 \\
\hline $\mathrm{H}$ & 0.85229 & 0.36664 & -5.79019 \\
\hline $\mathrm{H}$ & -0.86005 & -1.17251 & -6.68606 \\
\hline
\end{tabular}


${ }^{1} \mathrm{H}$ NMR (500MHz, $\left.\mathrm{CDCl}_{3}\right)$ and ${ }^{13} \mathrm{C}\{1 \mathrm{H}\}$ NMR $\left(\mathrm{CDCl}_{3}, 125 \mathrm{MHz}\right)$ spectrums of (3aR,5S,6R,6aR)-5-((R)2,2-dimethyl-1,3-dioxlan-4-yl)-6-(2-iodoethyl)-2,2-dimethyltetrahydrofuro [2,3-d][1,3]dioxole (4) $1 \mathrm{H}$ AMX500

SRK 4111-Iodination (rk0811 exp. 11; y.2010)

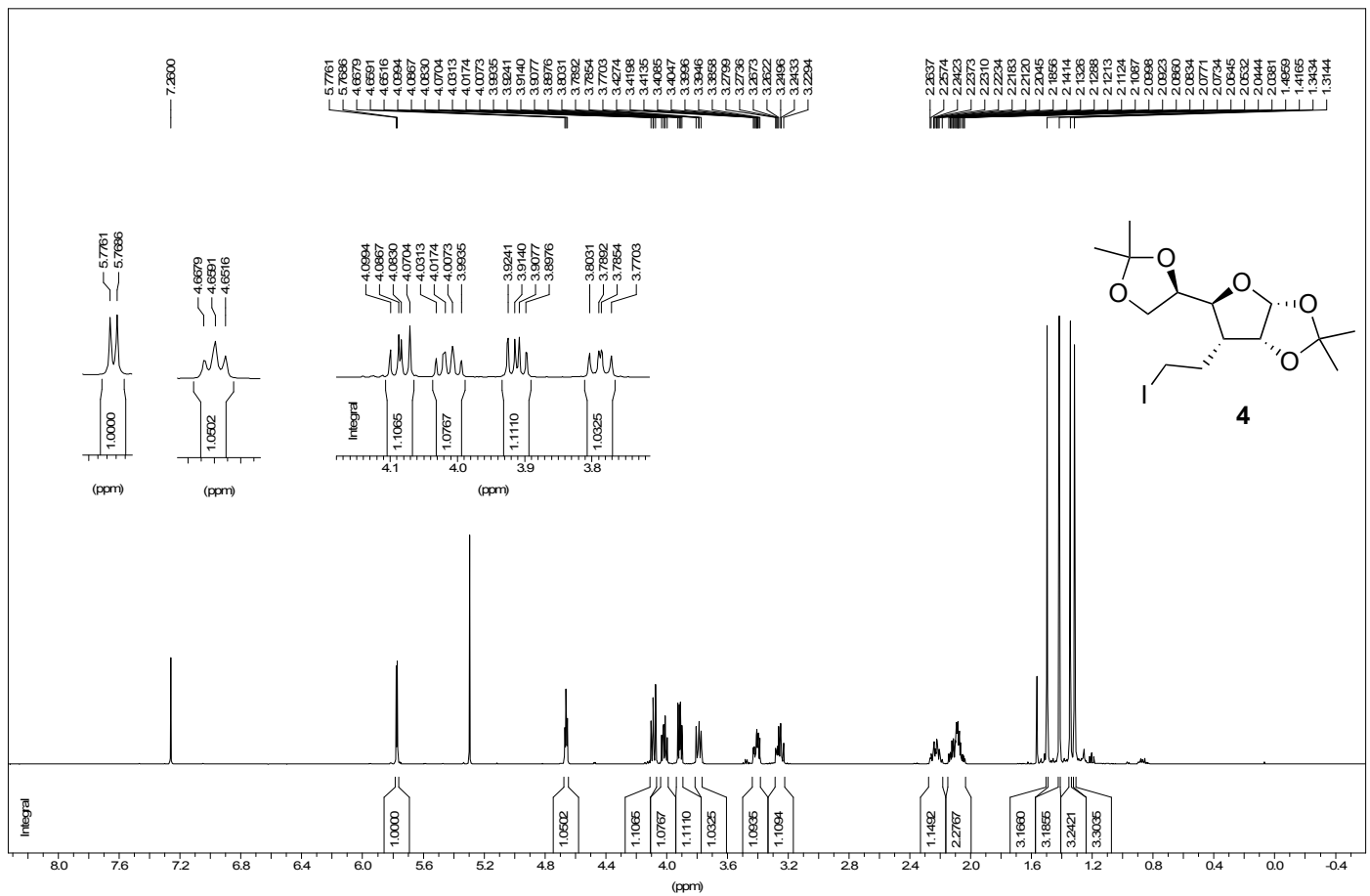

13C AMX500

SRK 4111-lodine (rk0811, exp 12; year 2010)

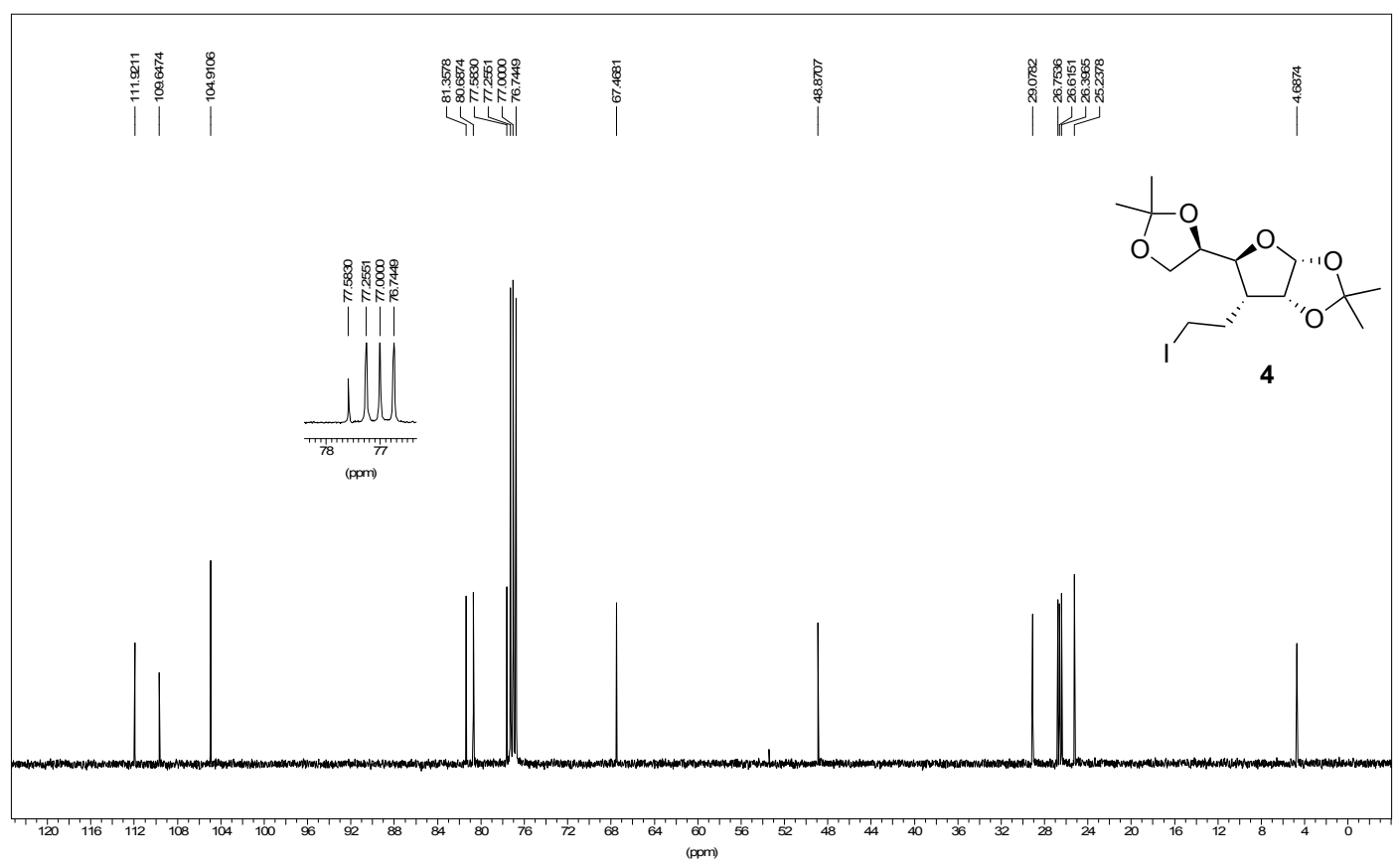


${ }^{1} \mathrm{H}$ NMR (500MHz, $\left.\mathrm{CDCl}_{3}\right)$ and ${ }^{13} \mathrm{C}\{1 \mathrm{H}\}$ NMR $\left(\mathrm{CDCl}_{3}, 125 \mathrm{MHz}\right)$ spectrums of (3aR,5S,6R,6aR)-6-(but-3en-1-yl)-5-((R)-2,2-dimethyl-1,3-dioxolan-4-yl)-2,2-dimethyltetrahydrofuro[2,3-d][1,3]dioxole (6):

4114_top spot

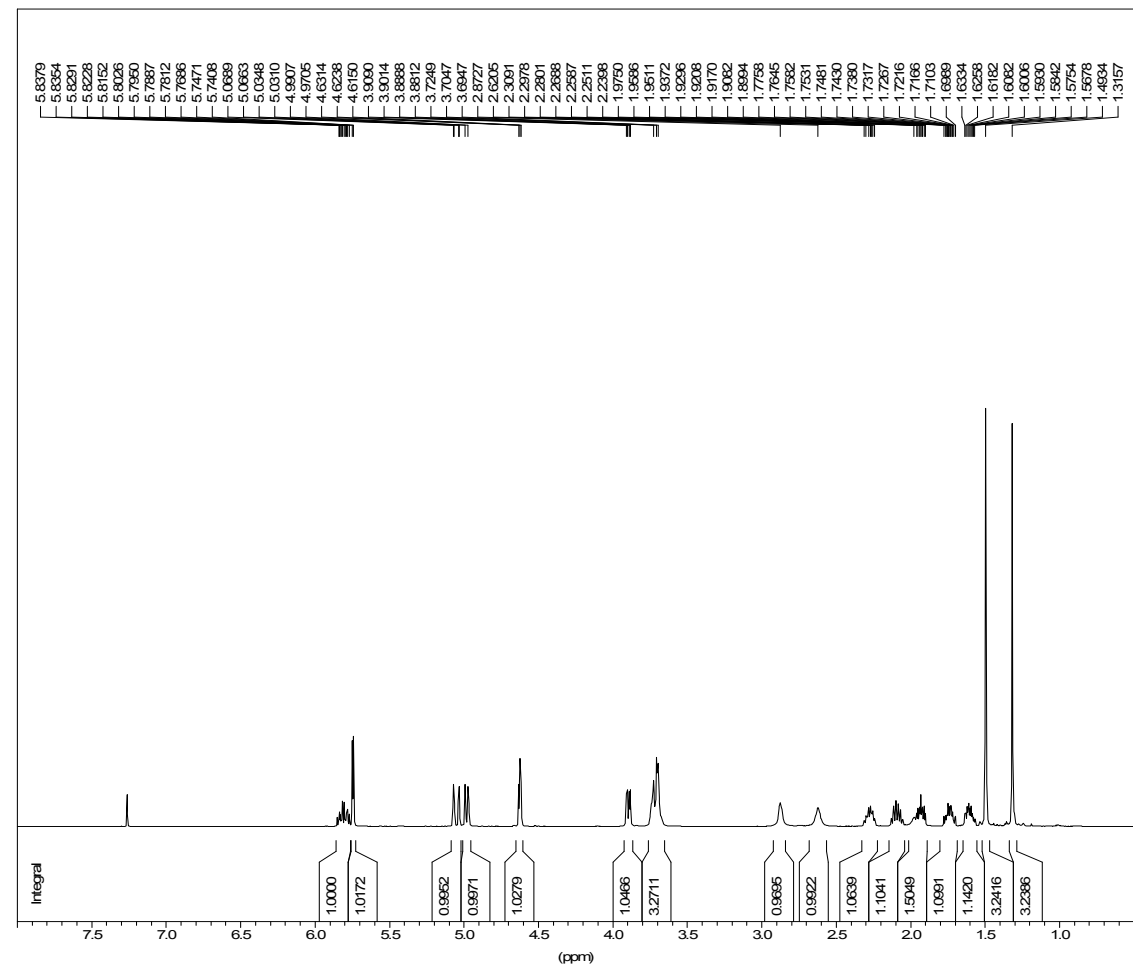

$$
\begin{aligned}
& \text { *** Current Data Parameters *** } \\
& \text { NAME : } \quad \text { rk0818 }
\end{aligned}
$$

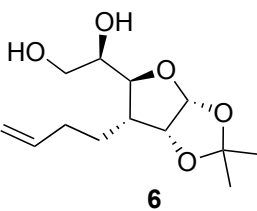

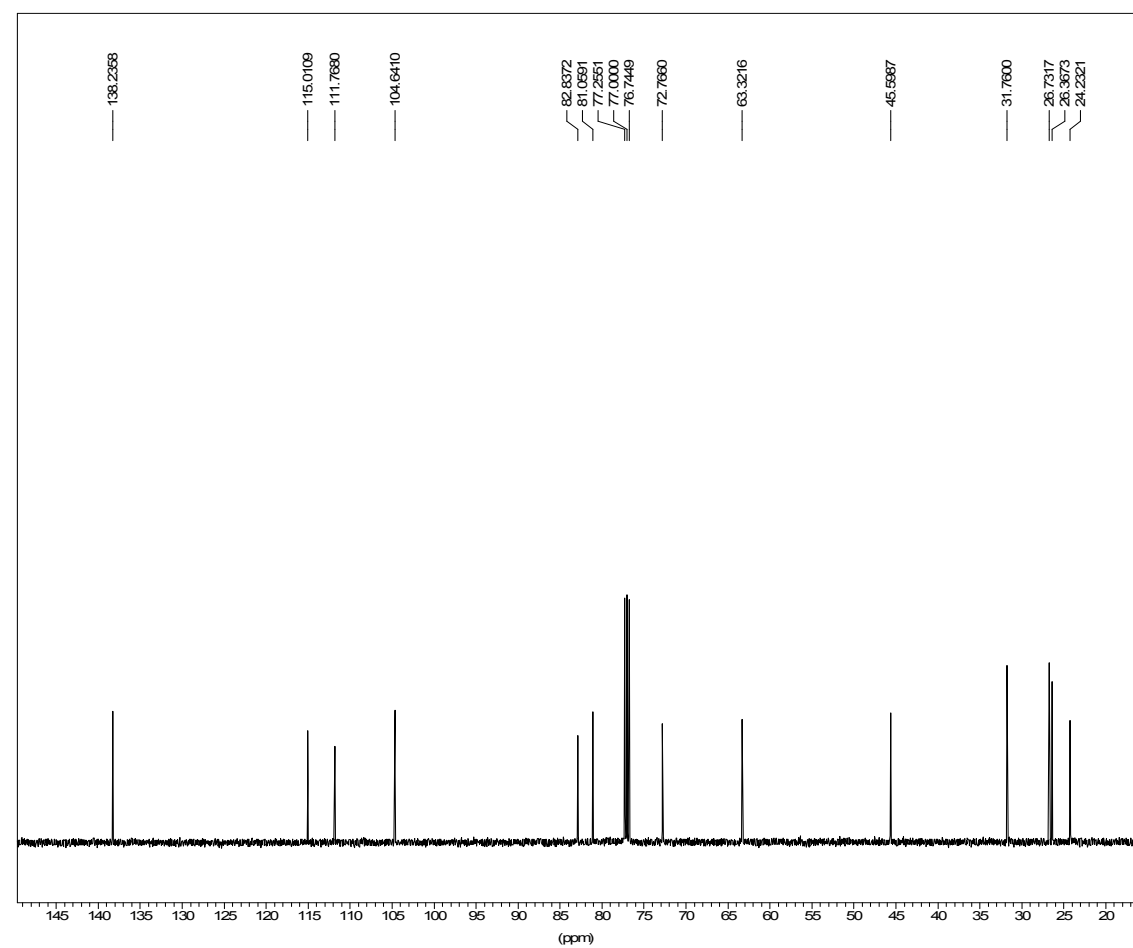

$$
\begin{aligned}
& { }^{* * *} \text { Current Data Parameters *** } \\
& \text { NAME : rk0818 } \\
& \text { EXPNO : } 12 \\
& \text { PROCNO }
\end{aligned}
$$

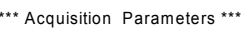

$$
\begin{aligned}
& \text { BF1 : } 125.7577890 \mathrm{MHz}
\end{aligned}
$$

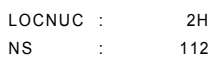

$$
\begin{aligned}
& 01: 13204.57 \mathrm{~Hz} \\
& \text { PULPROG: zgpg30 } \\
& \text { SFO1 : } 125.7709936 \mathrm{MHz} \\
& \text { SOLVENT : } \quad \mathrm{CDCl} \\
& \text { SW : } 238.7675 \mathrm{ppm} \\
& \text { *** Processing Parameters *** } \\
& \text { LB : } \quad 1.00 \mathrm{~Hz} \\
& \text { PHCO : } 162.833 \text { degree } \\
& \text { PHC1 : } 53.761 \text { degree }
\end{aligned}
$$

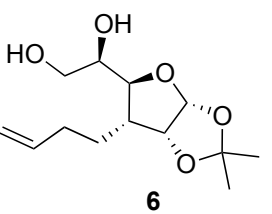


${ }^{1} \mathrm{H}$ NMR (500MHz, $\left.\mathrm{CDCl}_{3}\right)$ and ${ }^{13} \mathrm{C}\{1 \mathrm{H}\}$ NMR $\left(\mathrm{CDCl}_{3}, 125 \mathrm{MHz}\right)$ Spectrums of Methyl (E)-3-((3aR,5R,6R, 6aR)-6-(but-3-en-1-yl)-2,2-dimethyltetrahydrofuro[2,3-d][ 1,3]dioxol-5-yl)-2-methylacrylate (8):

$1 \mathrm{H}$ AMX500

4116_TT 3

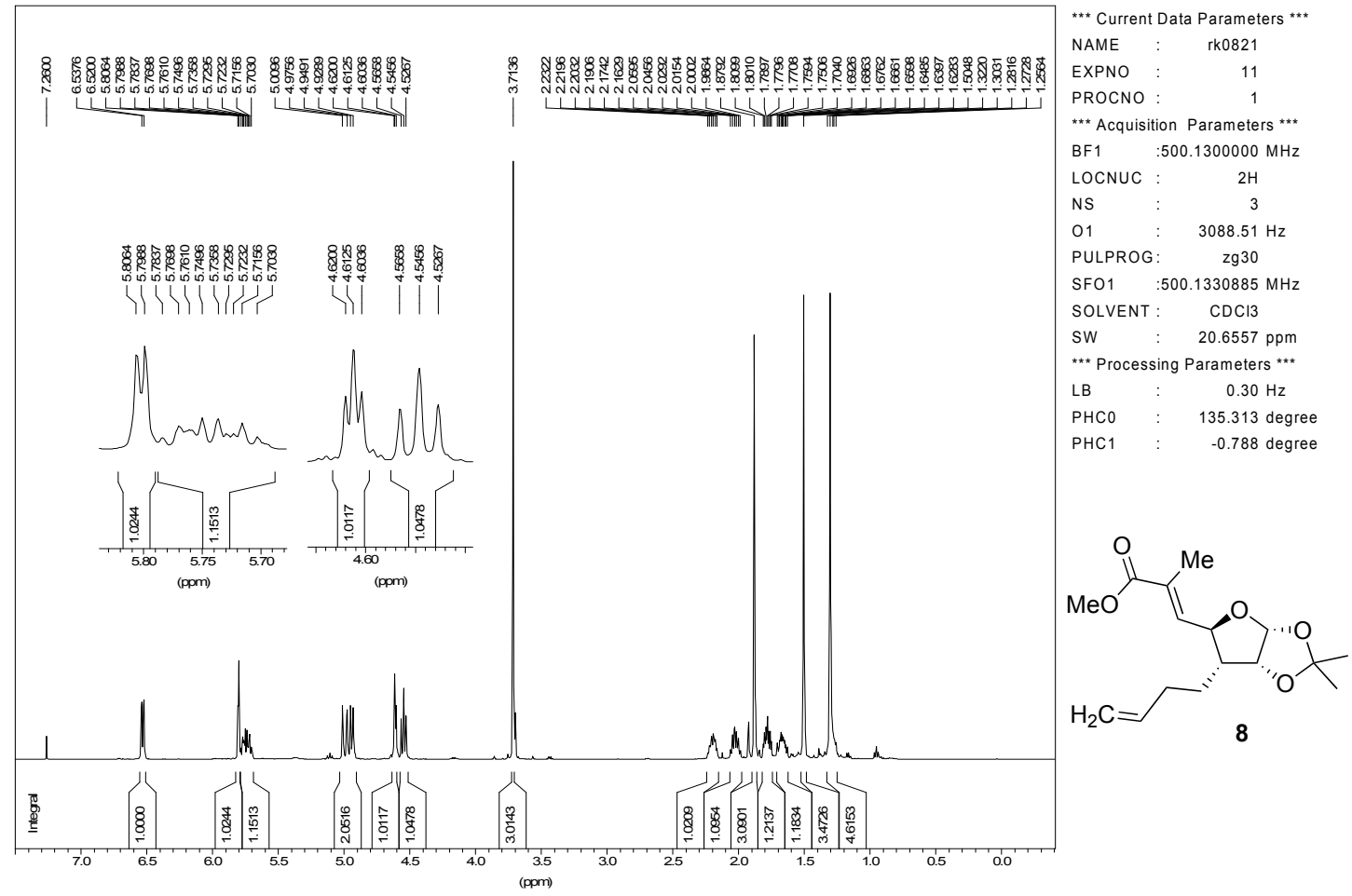

13C AMX500

4116_TT 3 (rk0821, exp. 12; year 2010)

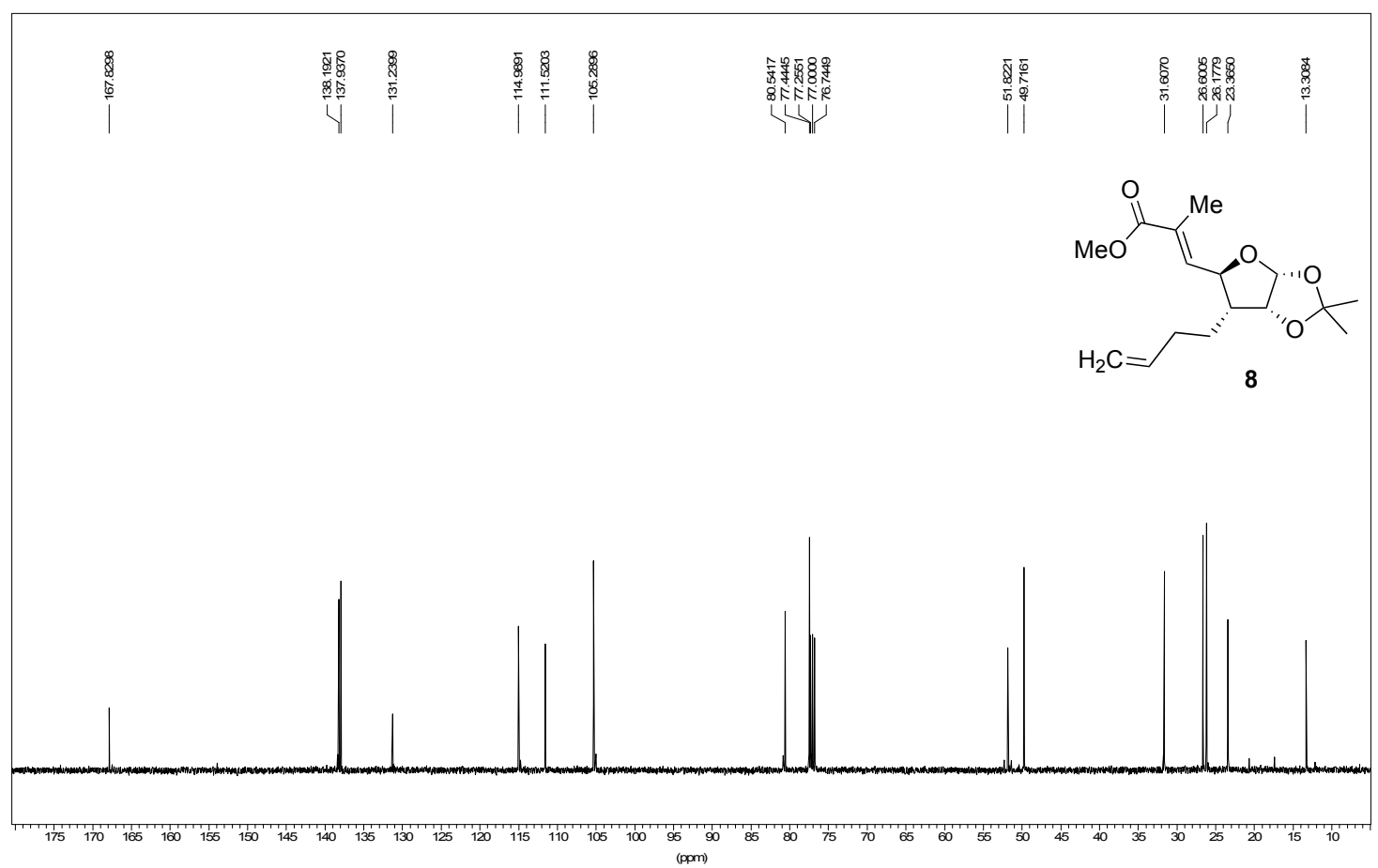


${ }^{1} \mathrm{H}$ NMR (500MHz, $\left.\mathrm{CDCl}_{3}\right)$ and ${ }^{13} \mathrm{C}\{1 \mathrm{H}\}$ NMR $\left(\mathrm{CDCl}_{3}, 125 \mathrm{MHz}\right)$ Spectrums of $(E)-3-((3 \mathrm{a} R, 5 R, 6 R, 6 \mathrm{a} R)-6-$ (but-3-en-1-yl)-2,2-dimethyltetrahydrofuro[2,3- $d][1,3]$ dioxol-5-yl)-2-methylprop-2-en-1-ol (9)

1H AMX500

TBAF_TBDPS removal_top spot_pure (rk0526 exp 5)

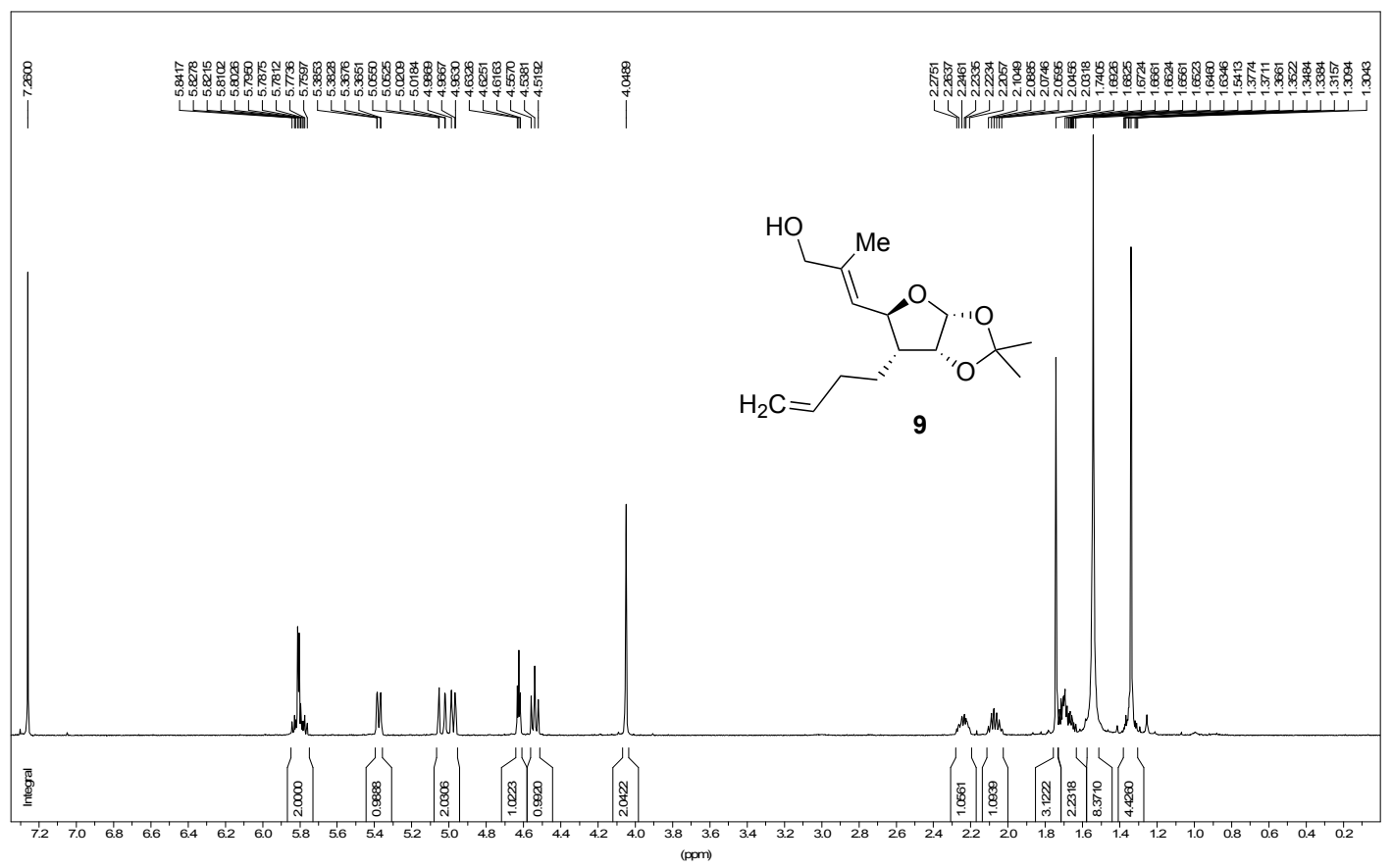

13C AMX500

TBAF_TBDPS removal_Top spot

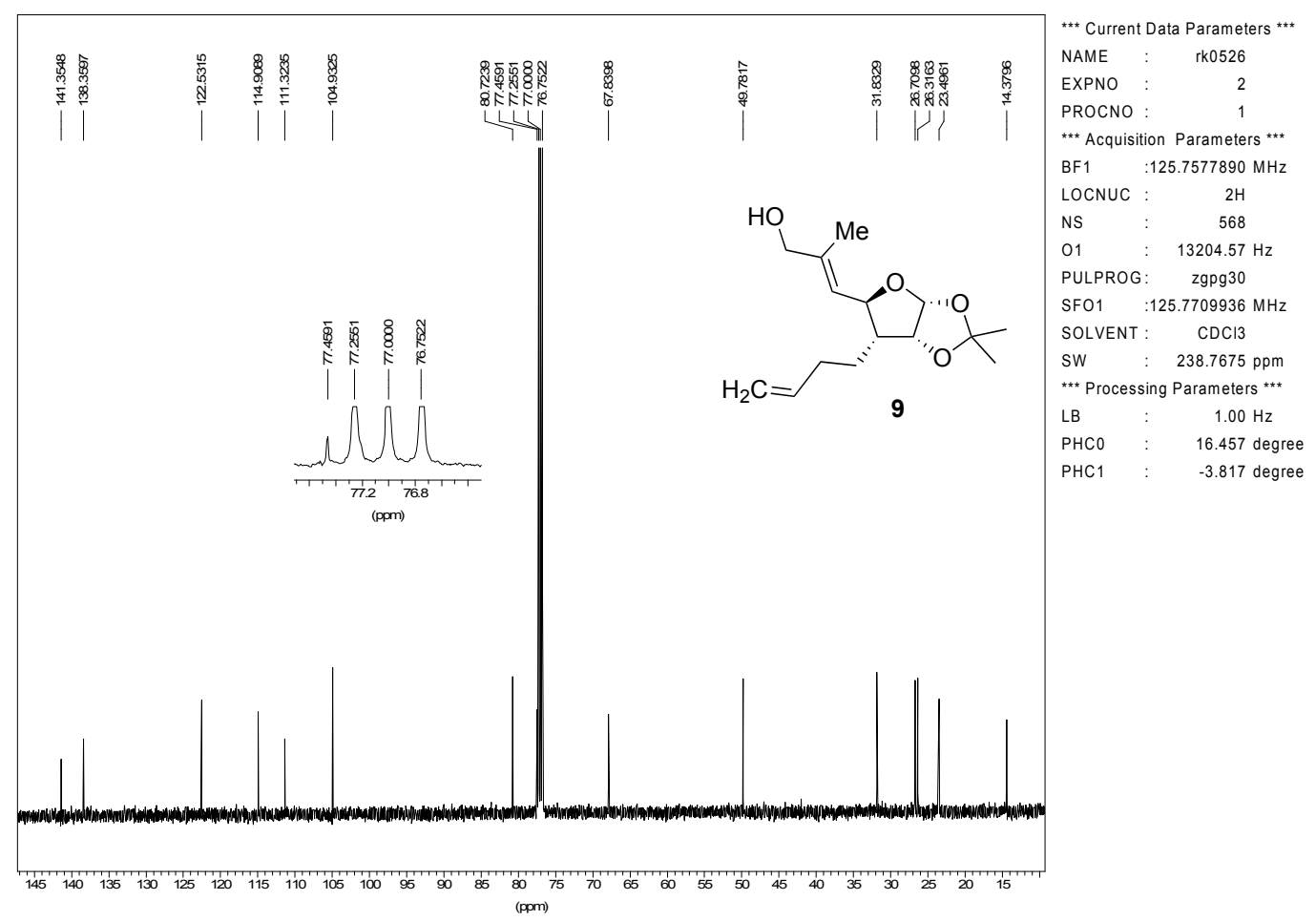


${ }^{1} \mathrm{H}$ NMR (500MHz, $\left.\mathrm{CDCl}_{3}\right)$ and ${ }^{13} \mathrm{C}\{1 \mathrm{H}\}$ NMR $\left(\mathrm{CDCl}_{3}, 125 \mathrm{MHz}\right)$ Spectrums of $(E)-3-((3 \mathrm{a} R, 5 R, 6 R, 6 \mathrm{a} R)-6-$ (But-3-enyl)-2,2-dimethyltetrahydrofuro[2,3- $d][1,3]$ dioxol -5-yl)-2-methylacrylaldehyde (10):

$1 \mathrm{H}$ AMX500

SRK $4097=$ right fragment (rk0610 exp 11)

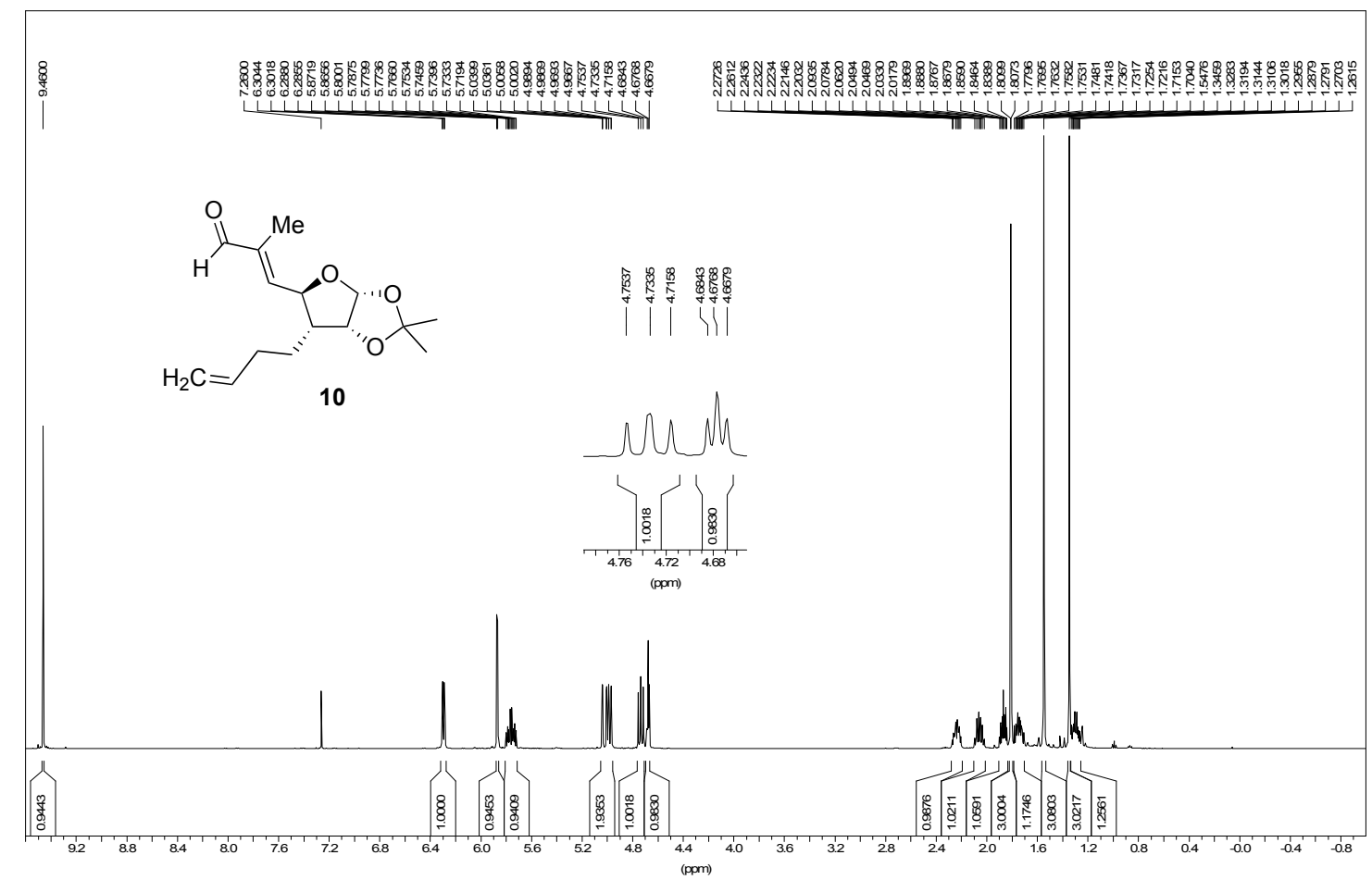

13C AMX500

SRK $4097=$ right fragment (rk0610 exp 12)

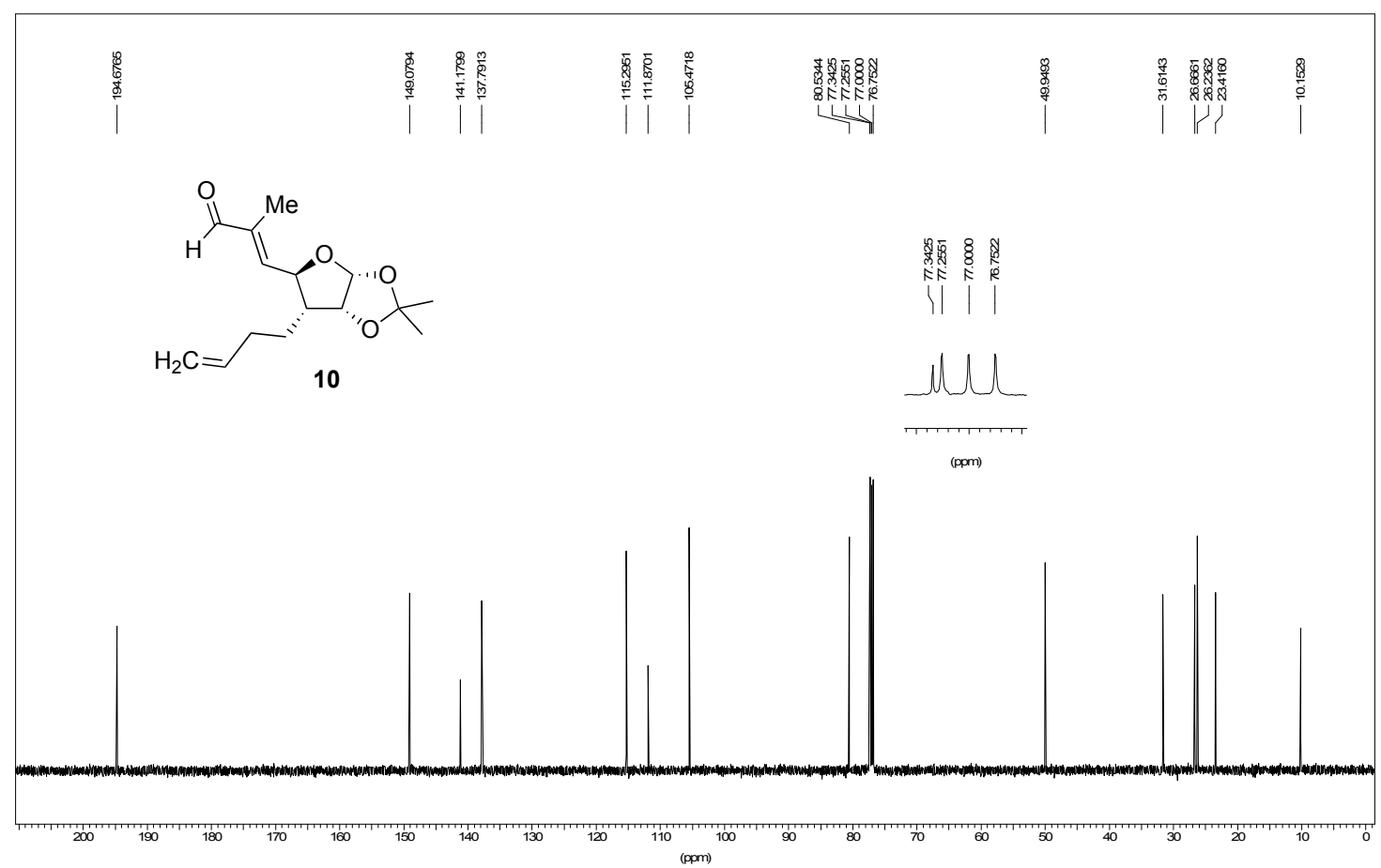


${ }^{1} \mathrm{H}$ NMR (500MHz, $\left.\mathrm{CDCl}_{3}\right)$ and ${ }^{13} \mathrm{C}\{1 \mathrm{H}\}$ NMR $\left(\mathrm{CDCl}_{3}, 125 \mathrm{MHz}\right)$ Spectrums of (2S,4S)-2-phenyl-4-vinyl1,3-dioxane (13)

1H AMX500

srk 4007_wittig pdt

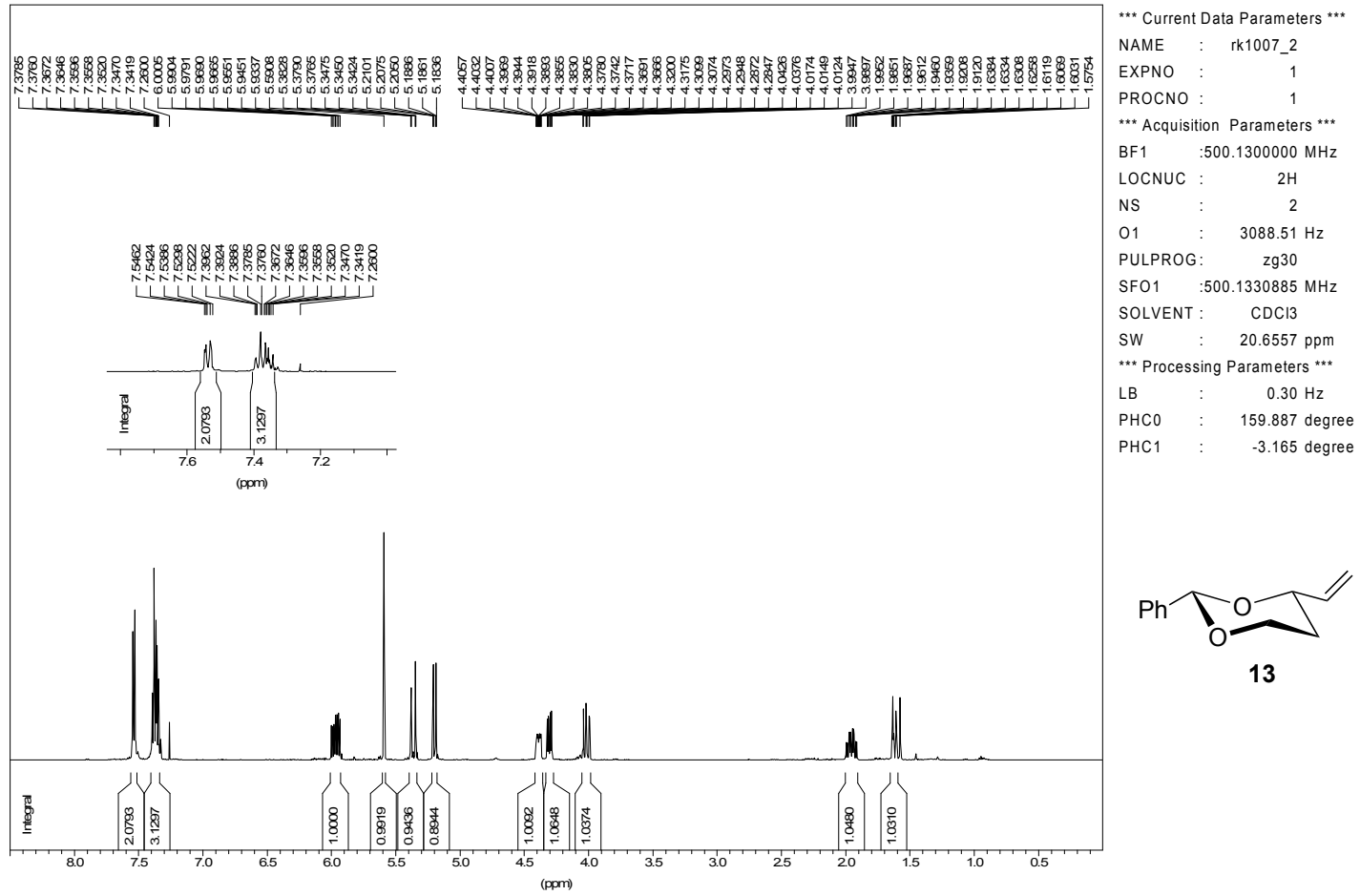

$13 \mathrm{C}$ AMX500

srk 4007_wittig pdt (rk1007_2 <y. 2009> exp 2 )

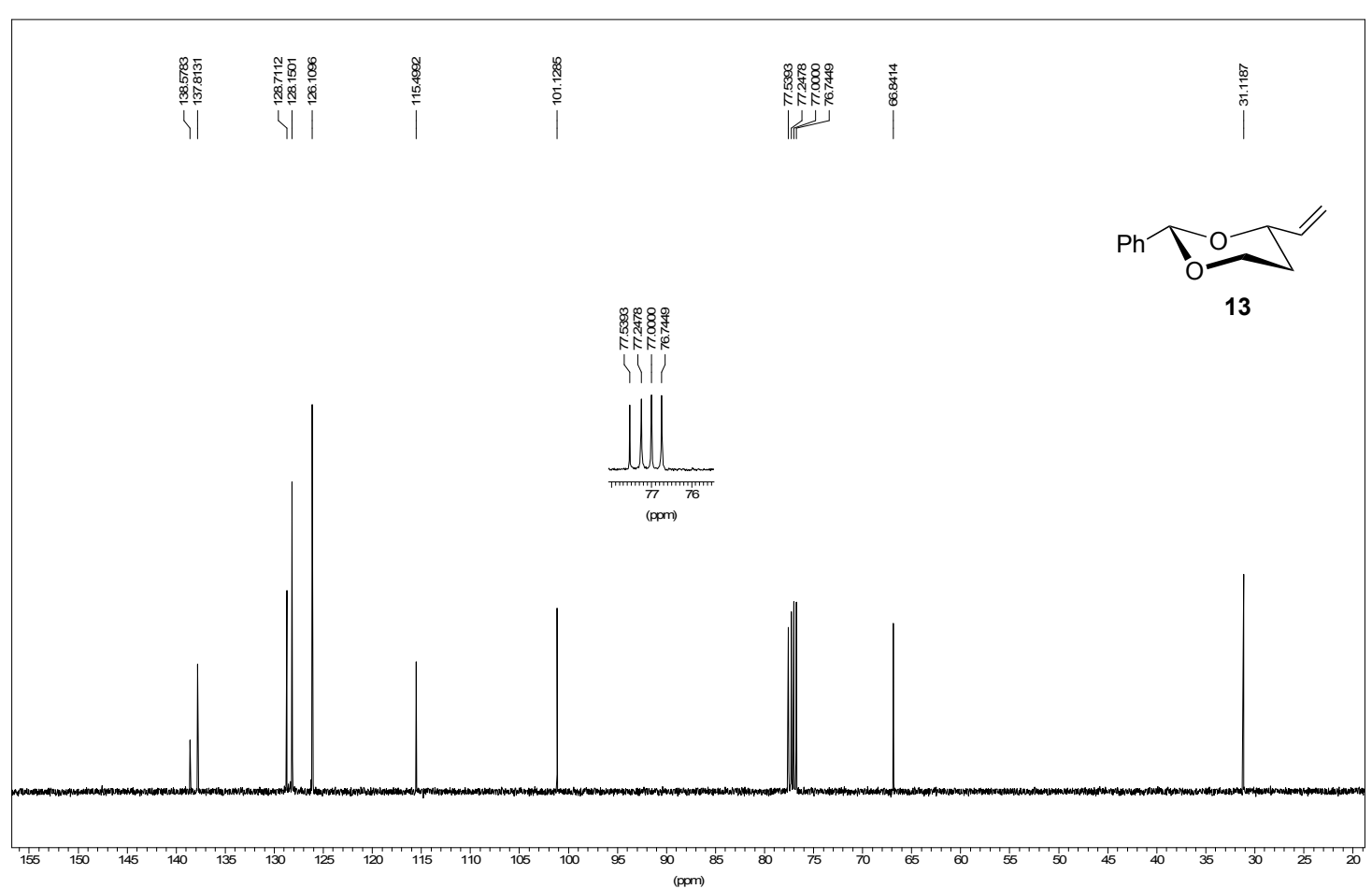


${ }^{1} \mathrm{H}$ NMR (500MHz, $\left.\mathrm{CDCl}_{3}\right)$ and ${ }^{13} \mathrm{C}\{1 \mathrm{H}\}$ NMR $\left(\mathrm{CDCl}_{3}, 125 \mathrm{MHz}\right)$ Spectrums of $(S)-3-($ benzyloxy$)$ pent-4en-1-ol (13a)

$1 \mathrm{H}$ AMX500

SRK 4009_spt 2_ benzylidene opening by LAH /AICI3

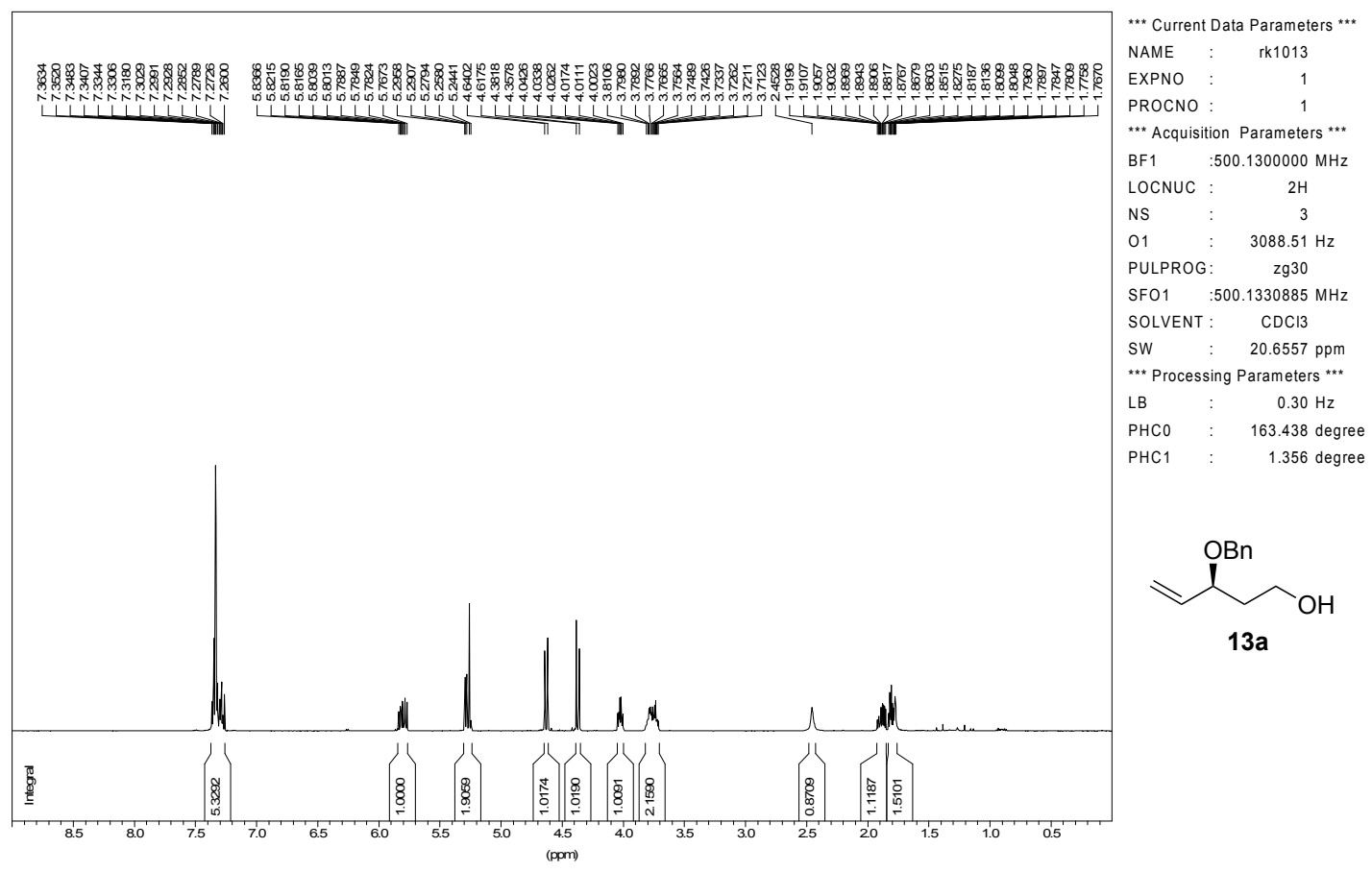

13C AMX500

SRK 4009_spt 2 benzylidene opening by LAH /AICI3 ( rk1013<y.2009> exp 2)

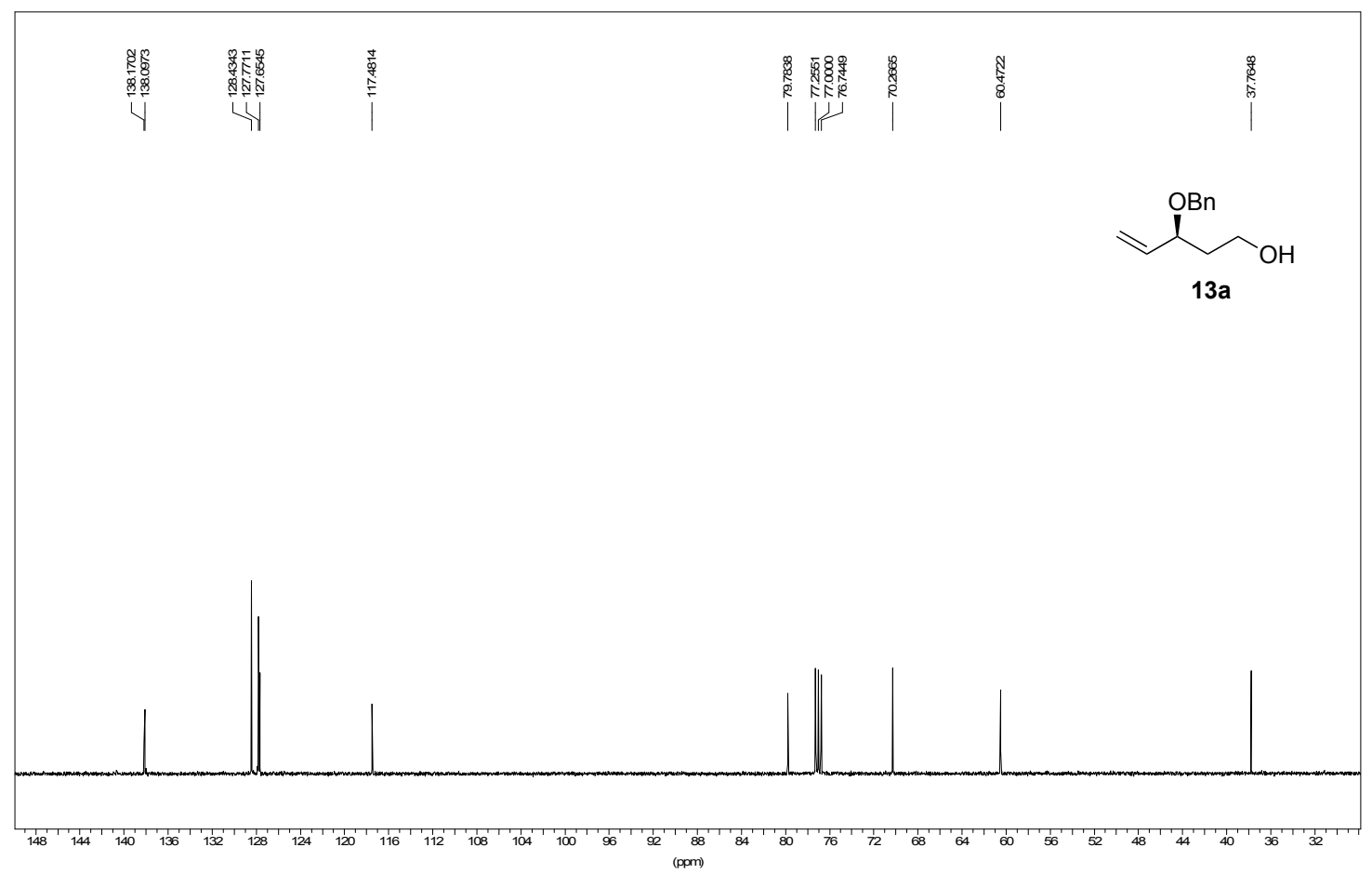


${ }^{1} \mathrm{H}$ NMR (500MHz, $\left.\mathrm{CDCl}_{3}\right)$ and ${ }^{13} \mathrm{C}\{1 \mathrm{H}\}$ NMR $\left(\mathrm{CDCl}_{3}, 125 \mathrm{MHz}\right)$ Spectrums of $(S)-3-($ benzyloxy)pent-4enal (14)

1 H AMX 500

SRK 4010_DMP oxdn

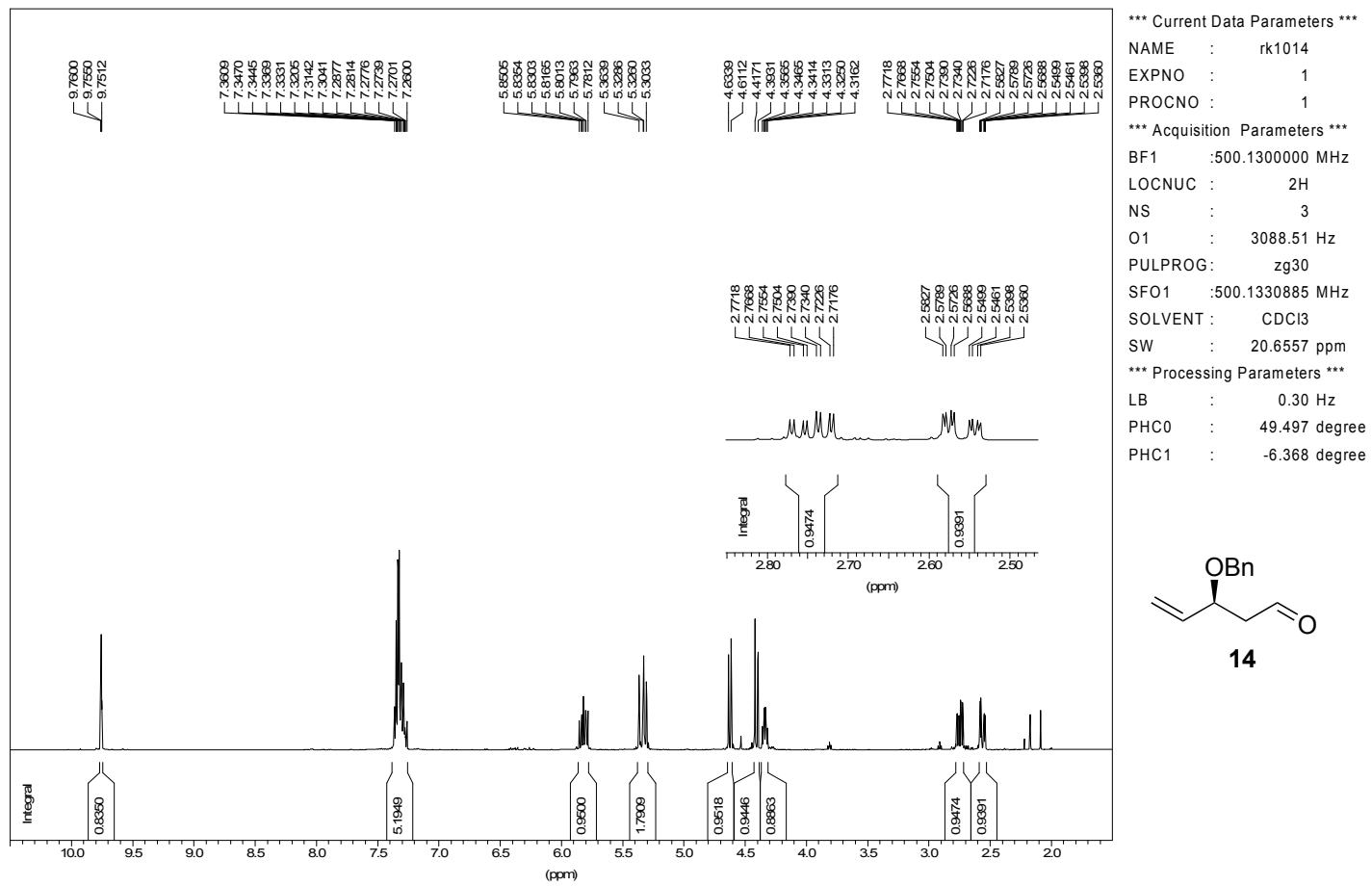

13C AMX500

SRK 4010_DMP oxdn (rk1014<2009> exp 2)

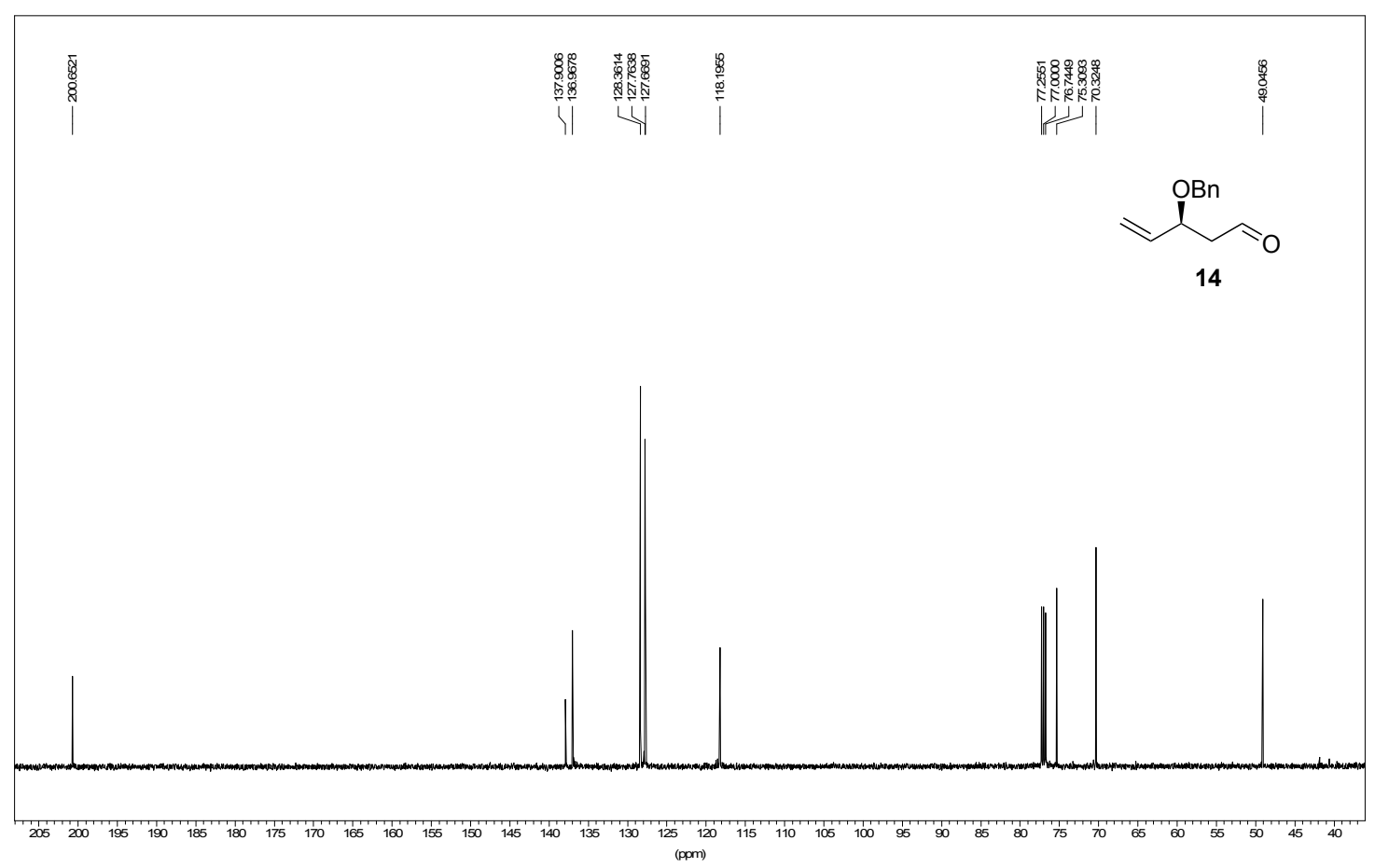


${ }^{1} \mathrm{H}$ NMR (500MHz, $\left.\mathrm{CDCl}_{3}\right)$ and ${ }^{13} \mathrm{C}\{1 \mathrm{H}\}$ NMR $\left(\mathrm{CDCl}_{3}, 125 \mathrm{MHz}\right)$ Spectrums of $(S)-4-($ benzyloxy)hex-5-en2-one (15)

1H AMX500

RSK4052 PCC

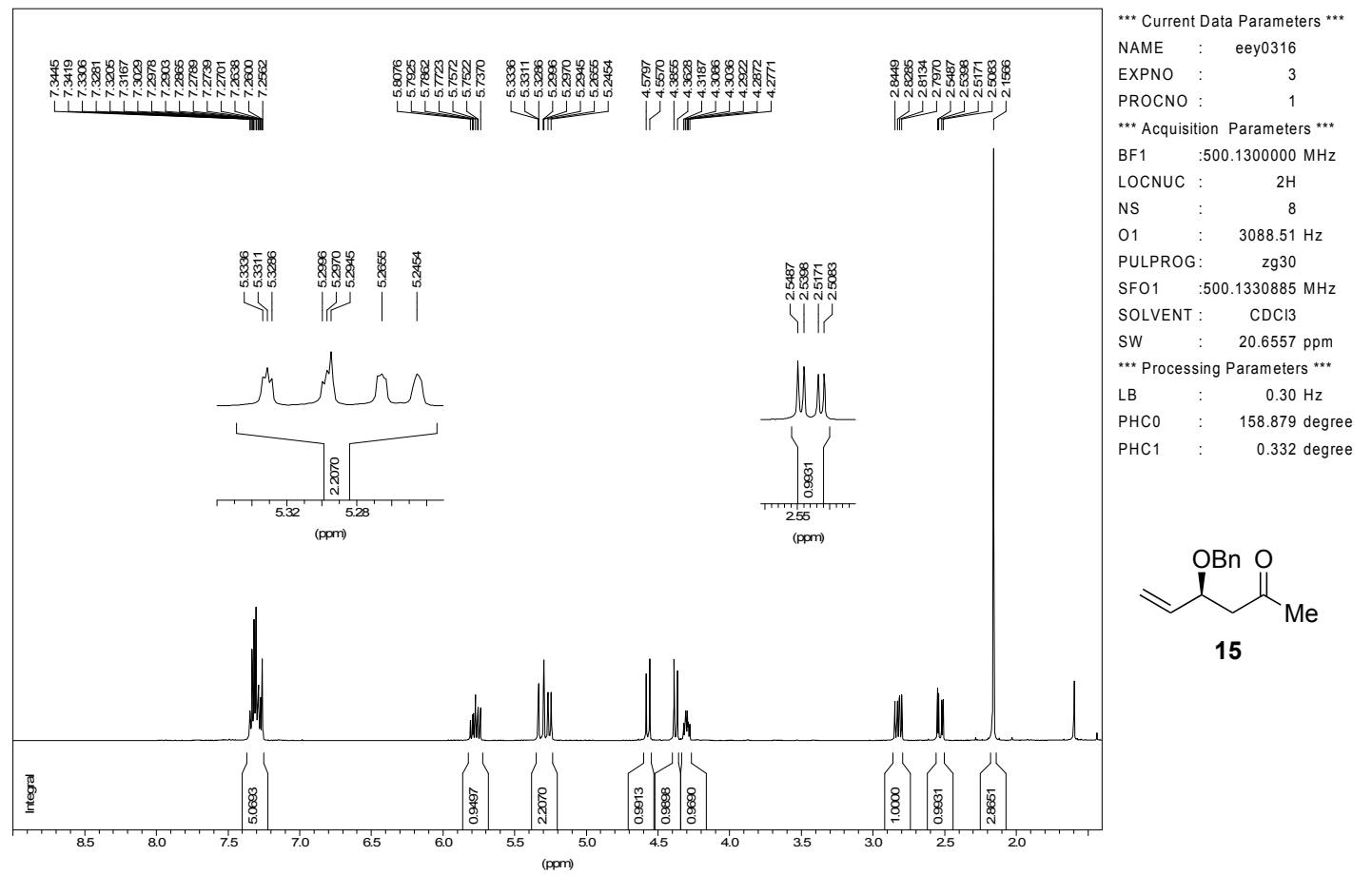

$13 \mathrm{C}$ Standard AC300

4052_PCC oxdn

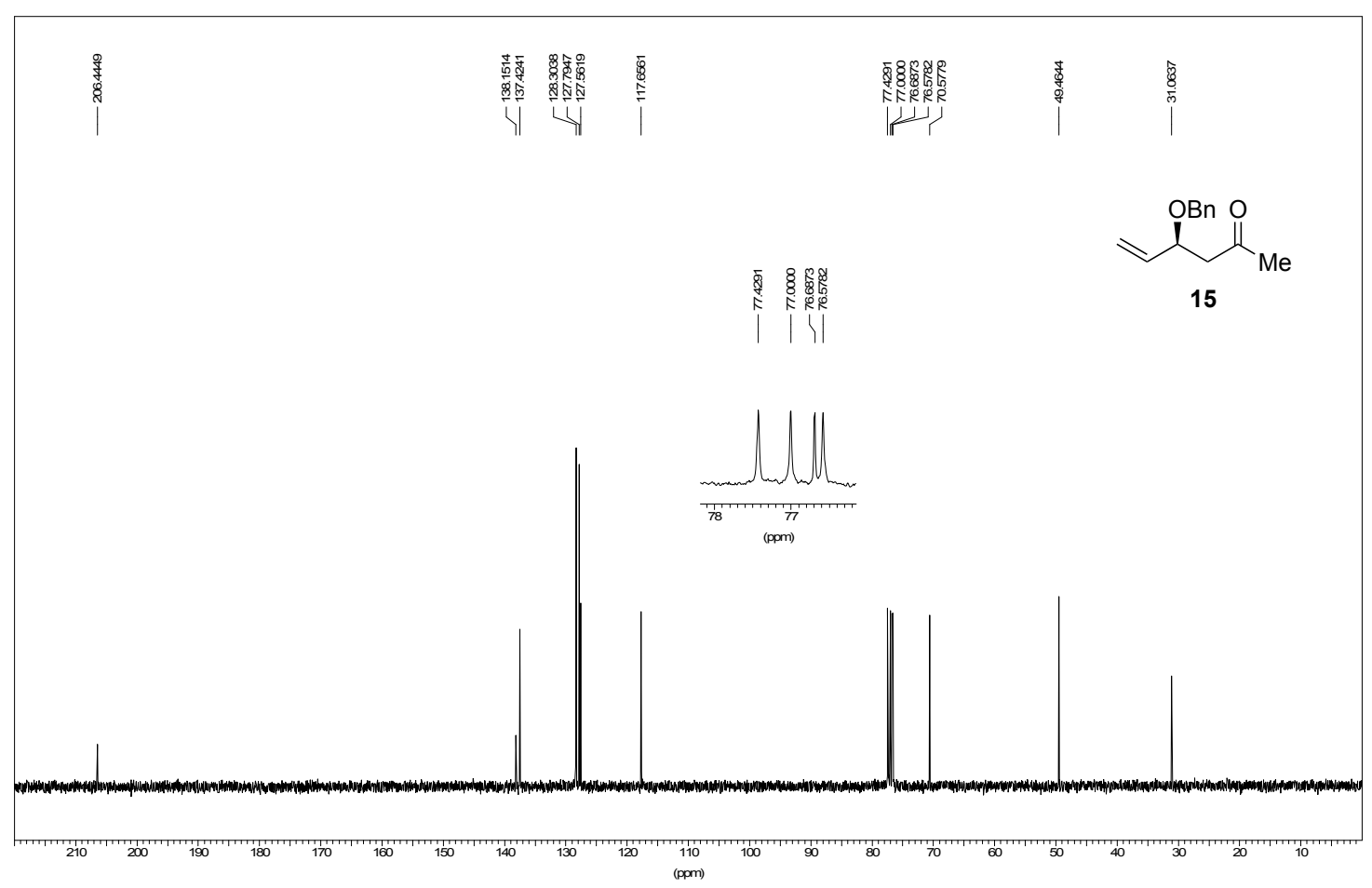


${ }^{1} \mathrm{H}$ NMR (500MHz, $\left.\mathrm{CDCl}_{3}\right)$ and ${ }^{13} \mathrm{C}\{1 \mathrm{H}\}$ NMR (CDCl $\left.3,125 \mathrm{MHz}\right)$ Spectrums of (5S)-5-(benzyloxy)-3methylhept-6-en-1-yn-3-ol (16)

1 AM $X 500$

4053_TOP_spot 1 (rk0317 exp 1)

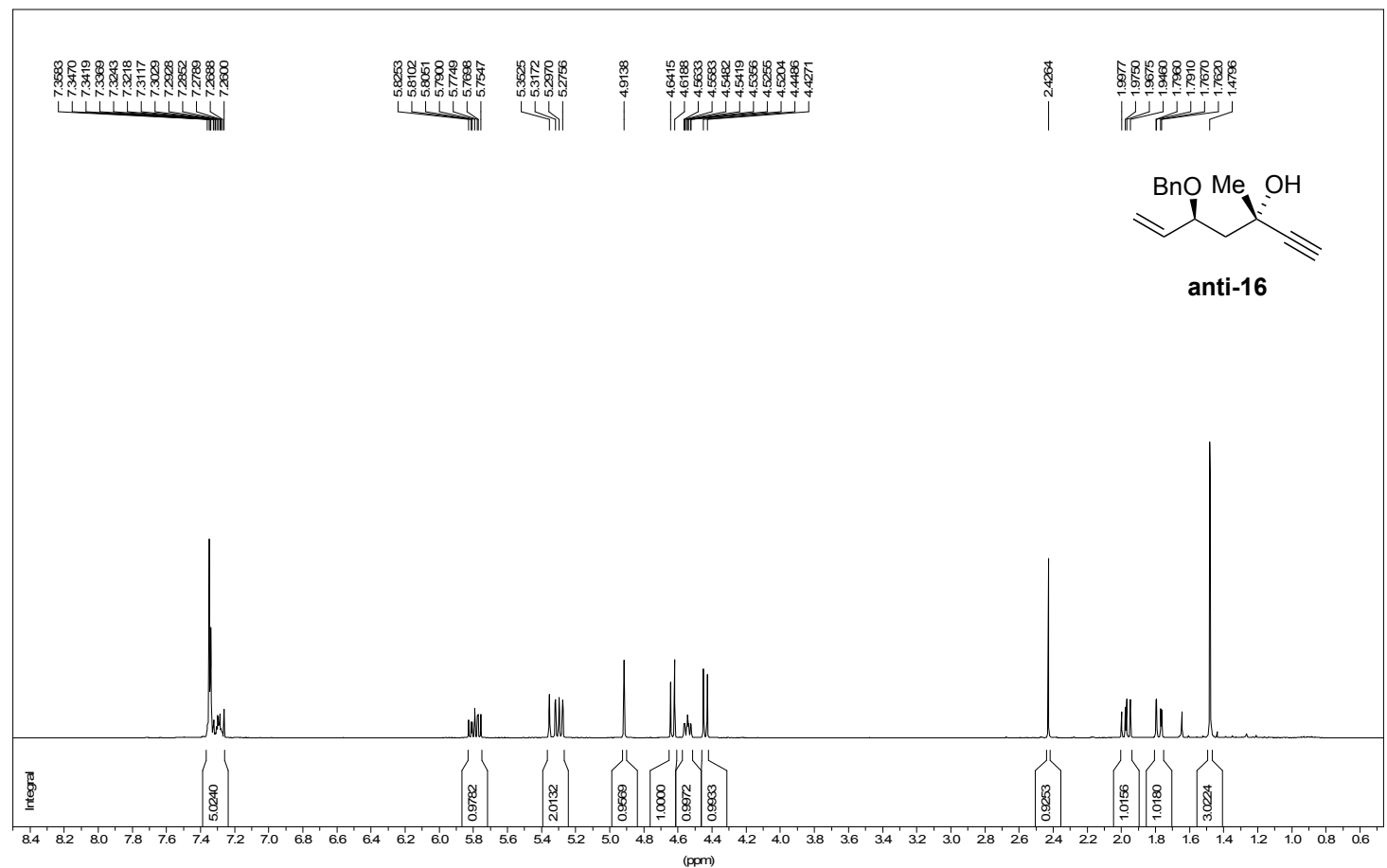

13C AMX500

4053_TOP_spot 1

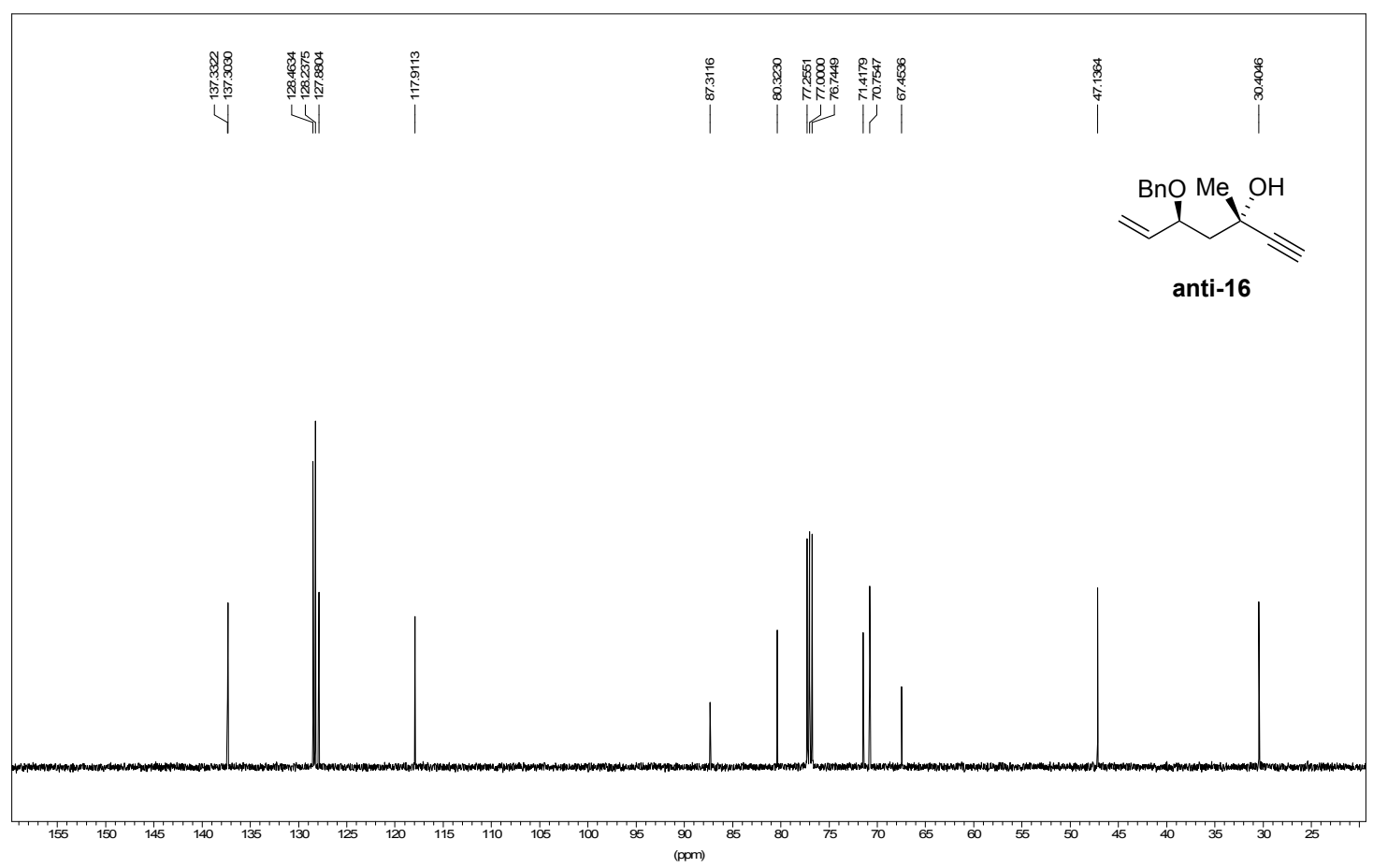


${ }^{1} \mathrm{H}$ NMR (500MHz, $\left.\mathrm{CDCl}_{3}\right)$ and ${ }^{13} \mathrm{C}\{1 \mathrm{H}\}$ NMR $\left(\mathrm{CDCl}_{3}, 125 \mathrm{MHz}\right)$ Spectrums of (3R,5S)-5-(benzyloxy)-3methylhept-6-en-1-yn-3-ol(16s)

$1 \mathrm{H}$ AMX500

4053_down_spot 3 (rk0317 exp 11)

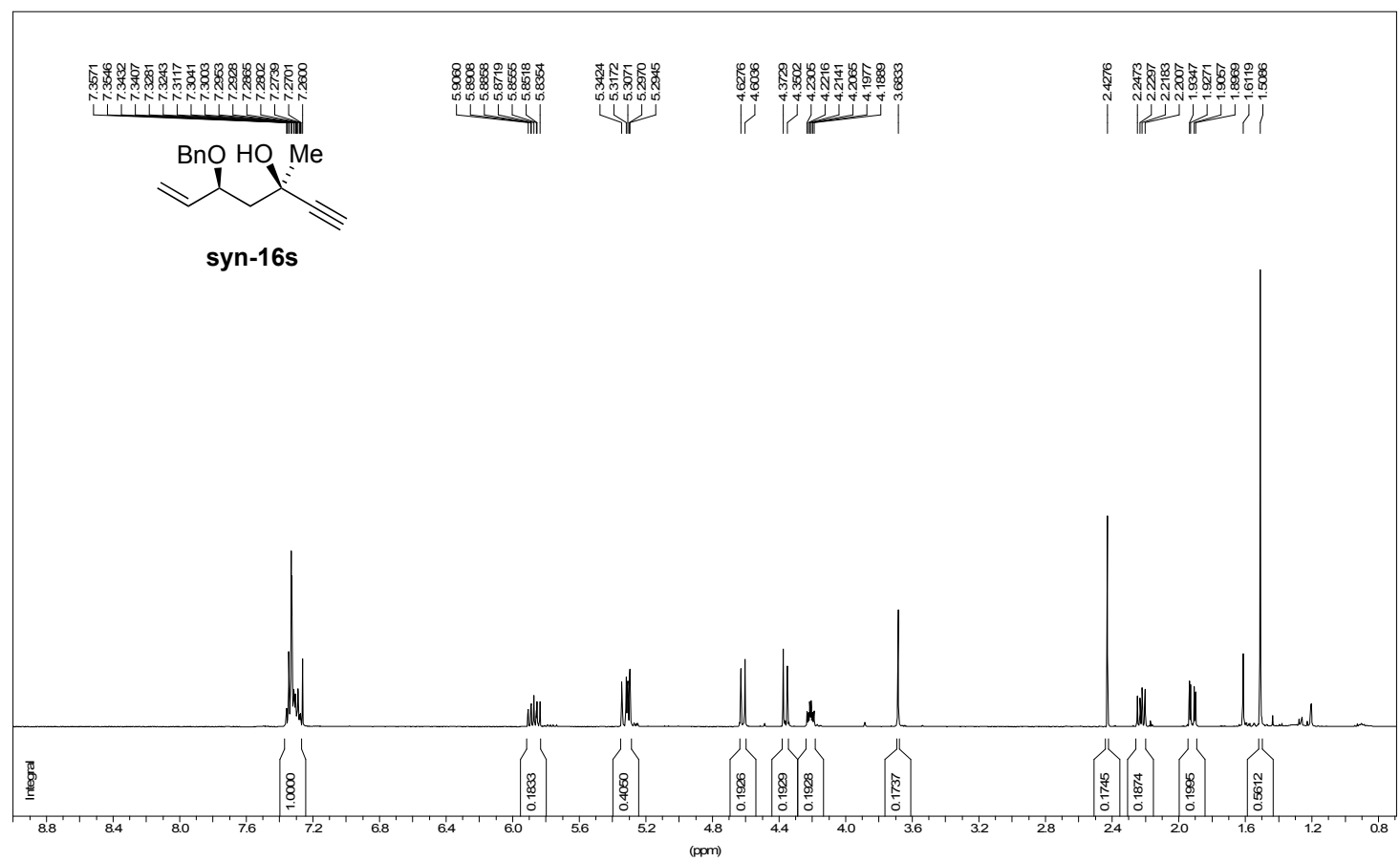

13C AMX500

SRK 4053_down_spot 3 (rk0317 exp 12)

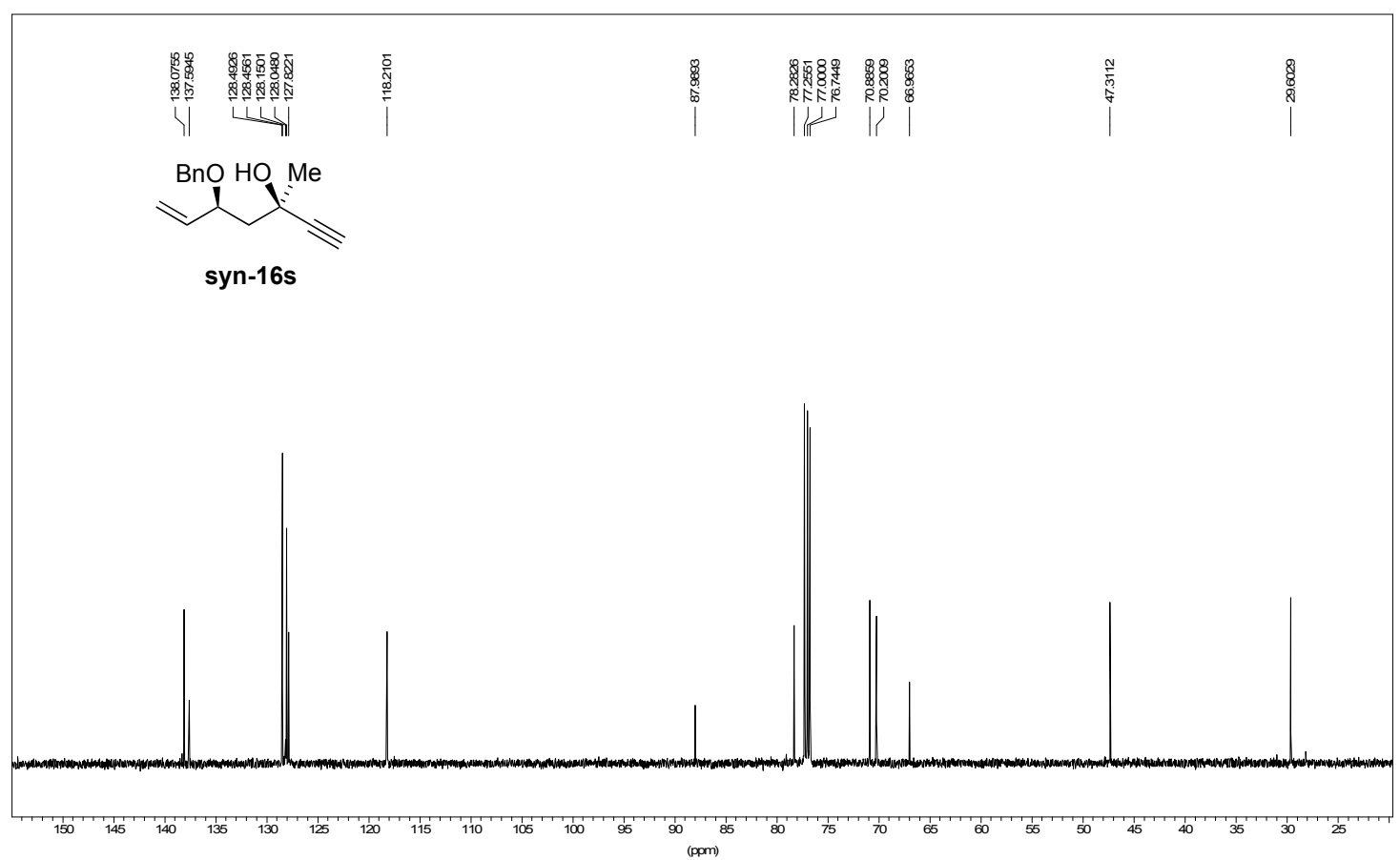


${ }^{1} \mathrm{H}$ NMR (500MHz, $\left.\mathrm{CDCl}_{3}\right)$ and ${ }^{13} \mathrm{C}\{1 \mathrm{H}\}$ NMR $\left(\mathrm{CDCl}_{3}, 125 \mathrm{MHz}\right)$ Spectrums of ((3S,5S)-5-(benzyloxy)-3methylhept-6-en-1-yn-3-yloxy)trimethylsilane (17)

1 H AMX500

SRK 4056_TMS Protection of tert-OH (rk 0322, exp 1)

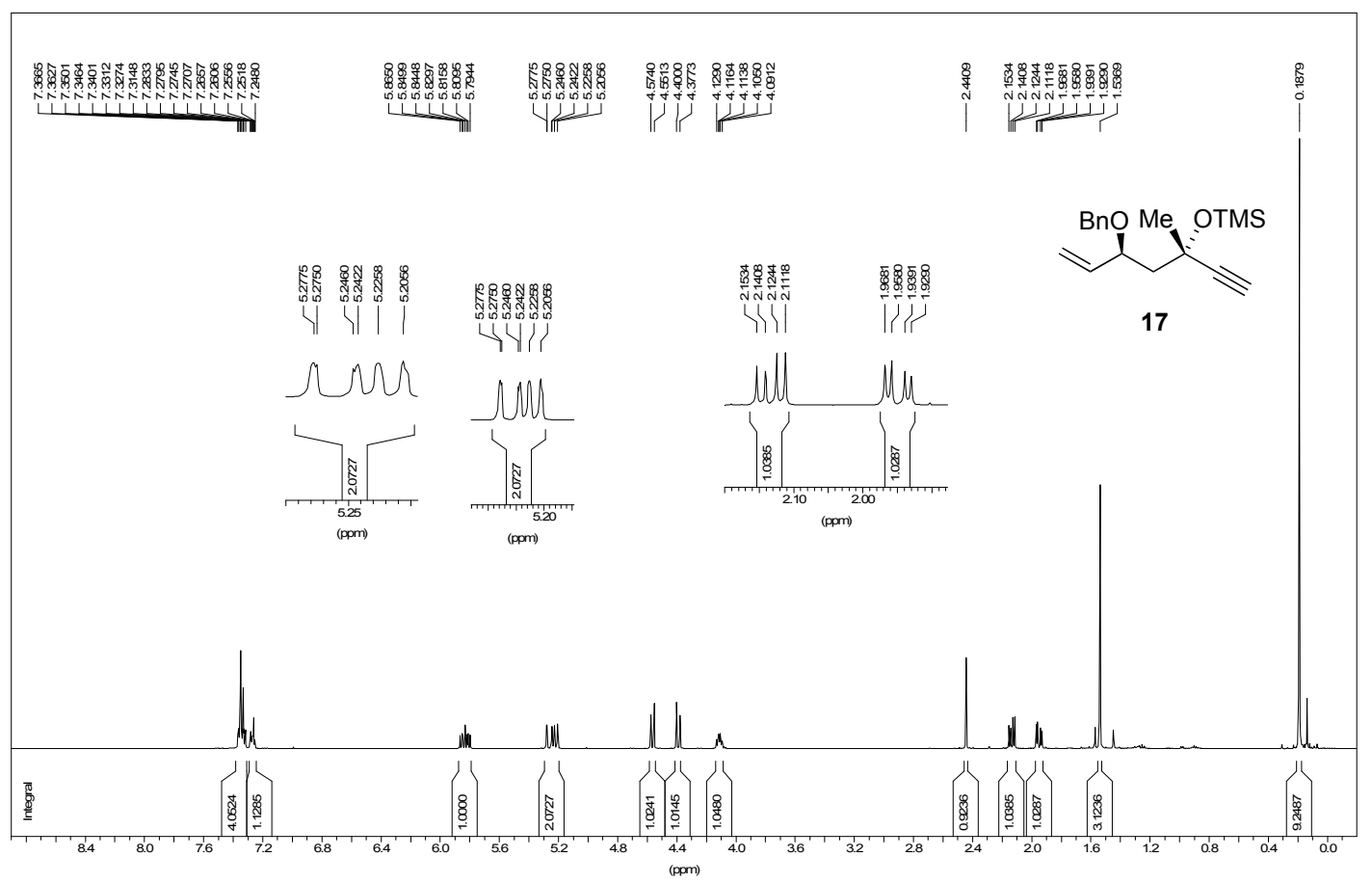

13C AMX500

SRK 4056 TMS Protection of tert alc (rk0322 exp.2)

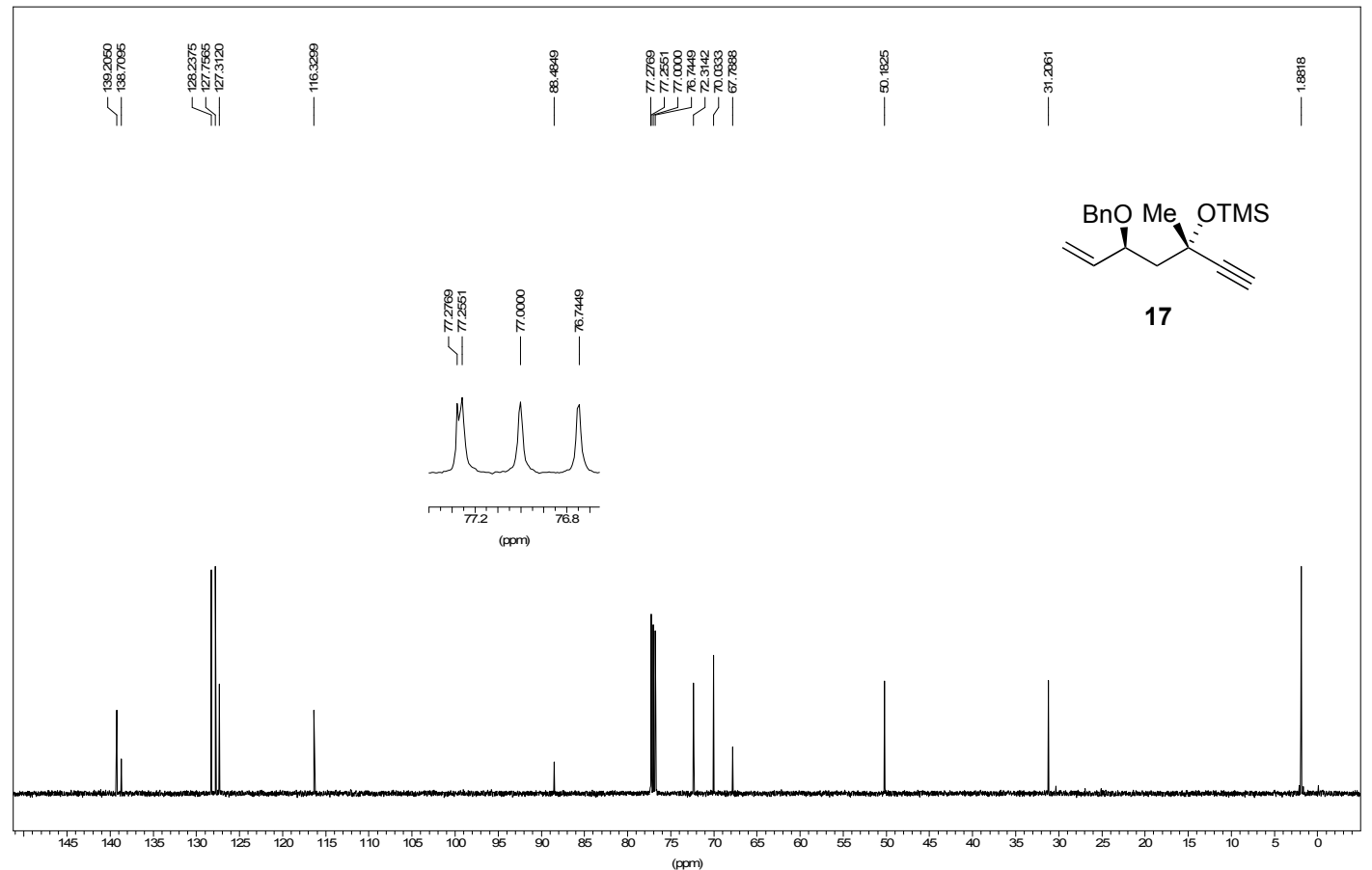


${ }^{1} \mathrm{H}$ NMR (500MHz, $\left.\mathrm{CDCl}_{3}\right)$ and ${ }^{13} \mathrm{C}\{1 \mathrm{H}\}$ NMR $\left(\mathrm{CDCl}_{3}, 125 \mathrm{MHz}\right)$ Spectrums of $(3 R, 6 S, 8 S, E)-8$-(benzyloxy)1-((3aR,5R,6R,6aR)-6-(but-3-enyl)-2,2-dimethyltetrahydrofuro[2,3- $d]$ [1,3]dioxol-5-yl)-2,6-dimethyl-6-(tri methylsilyloxy)deca-1,9-dien-4-yn-3-ol (18a)

1H AMX500

SRK 4100 Coupling_spot 1 (top) (rk0618 exp 1)

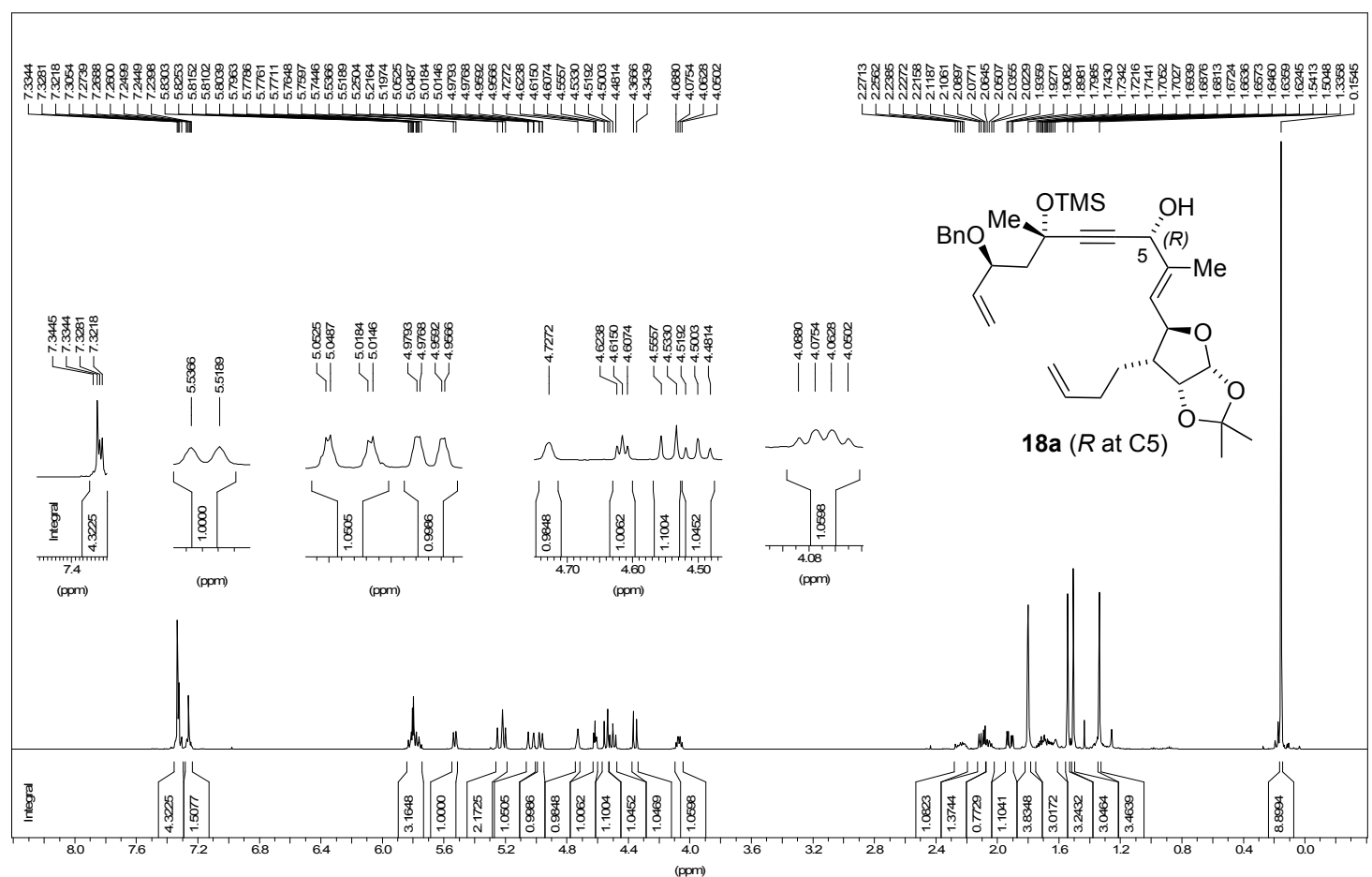

$13 \mathrm{C}$ AMX500

SRK 4100_Coupling_spot 1 (top)
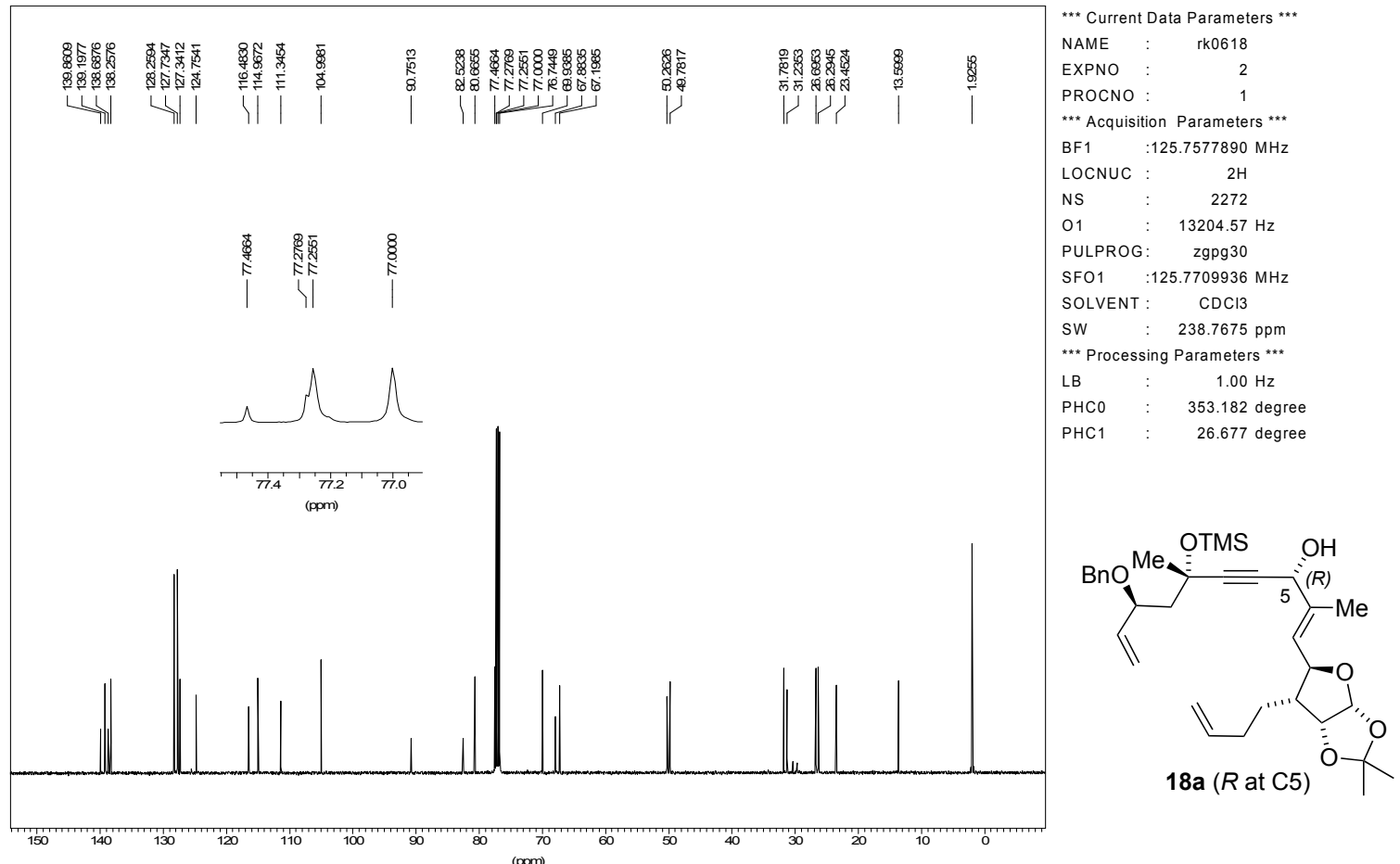
${ }^{1} \mathrm{H}$ NMR (500MHz, $\left.\mathrm{CDCl}_{3}\right)$ and ${ }^{13} \mathrm{C}\{1 \mathrm{H}\}$ NMR $\left(\mathrm{CDCl}_{3}, 125 \mathrm{MHz}\right)$ Spectrums of $(3 S, 6 S, 8 S, E)-8$-(Benzyloxy)1-((3aR,5R,6R,6aR)-6-(but-3-enyl)-2,2-dimethyltetrahydrofuro[2,3- $d]$ [1,3]dioxol-5-yl)-2,6-dimethyl-6-(tri methylsilyloxy)deca-1,9-dien-4-yn-3-ol (18b)

1H AMX500

$4100 \_$btm_pure
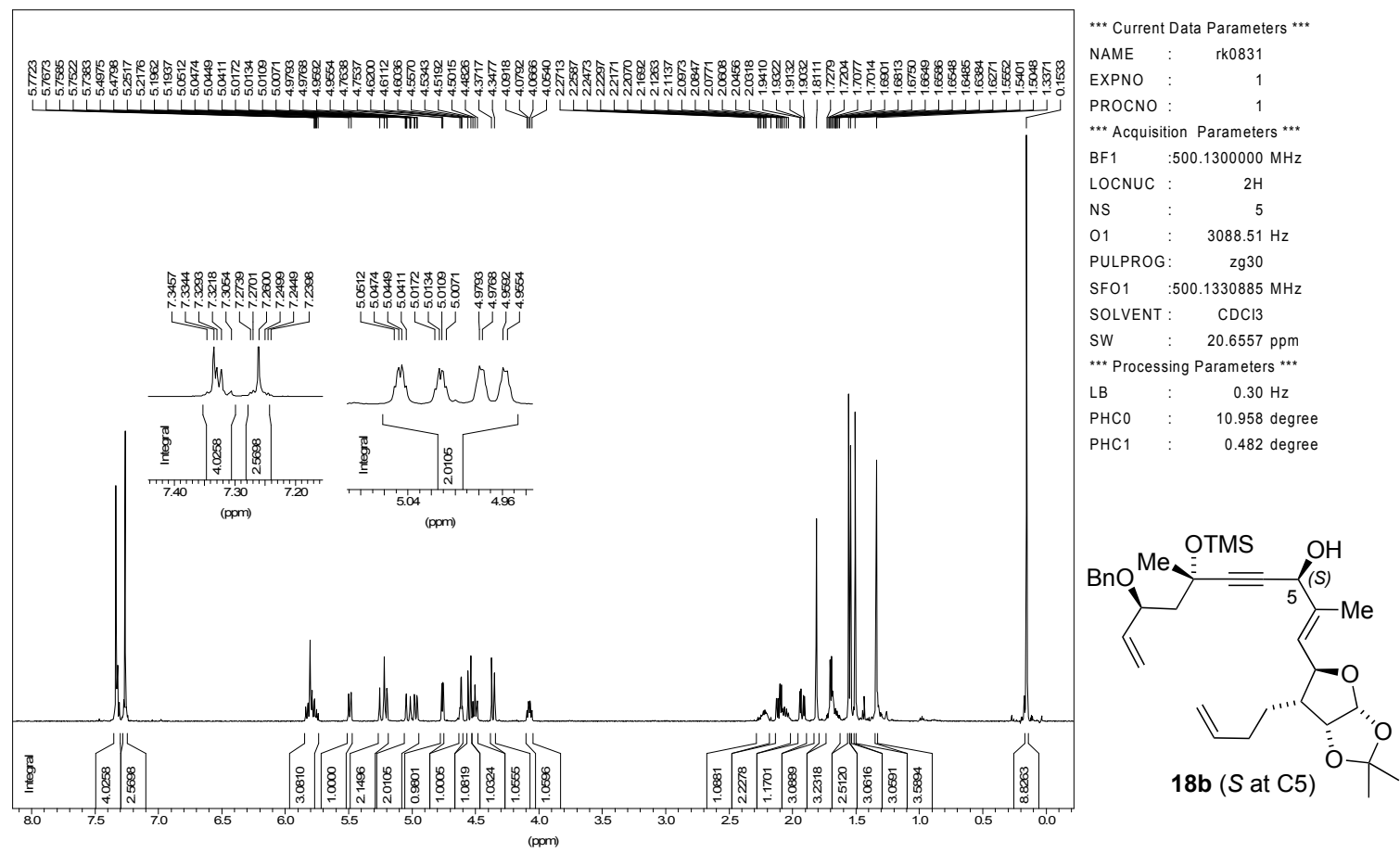

$13 \mathrm{C}$ AMX500

SRK 4100_Coupling_spot 2 (bottom)
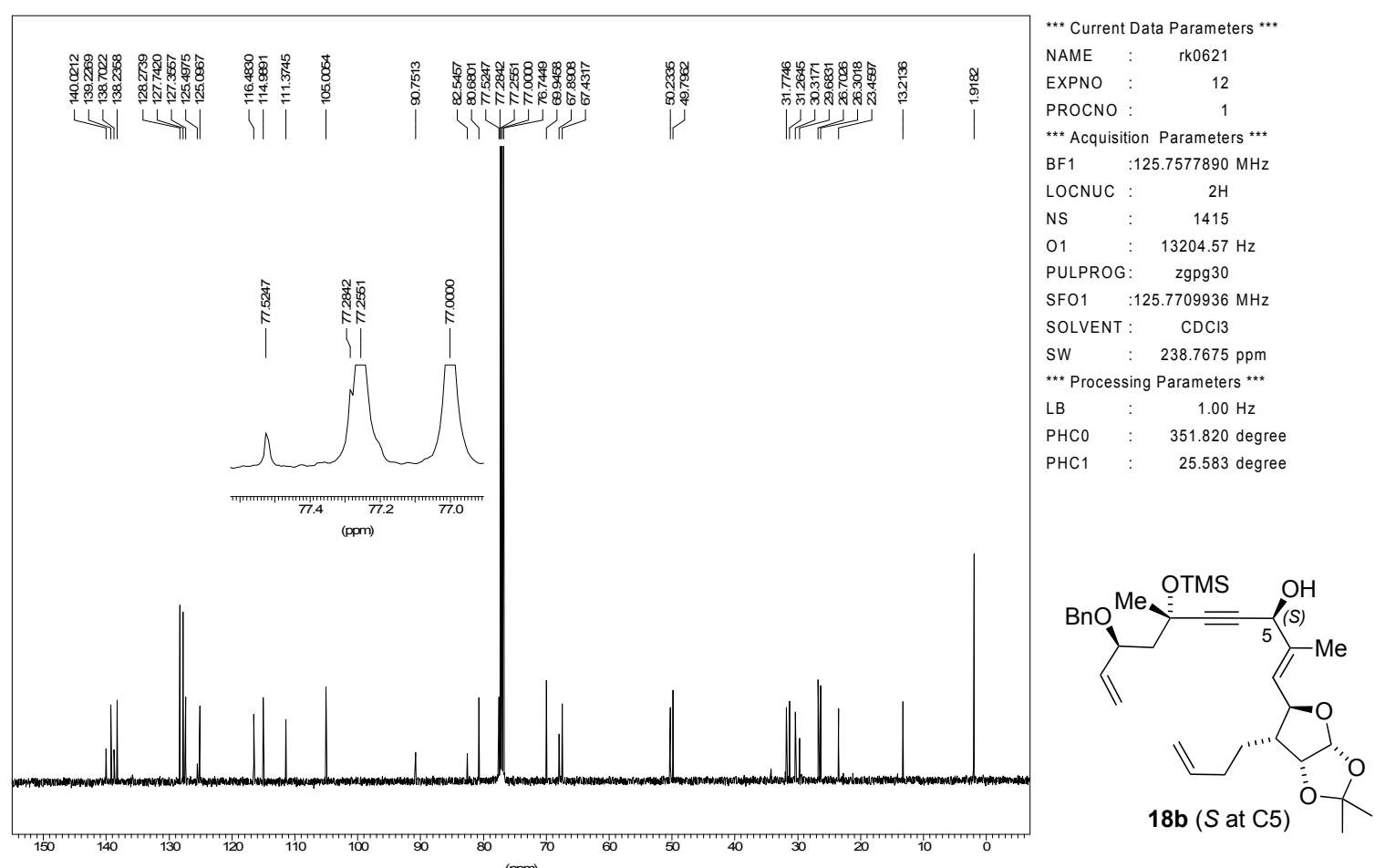
${ }^{1} \mathrm{H}$ NMR (500MHz, $\left.\mathrm{CDCl}_{3}\right)$ and ${ }^{13} \mathrm{C}\{1 \mathrm{H}\}$ NMR $\left(\mathrm{CDCl}_{3}, 125 \mathrm{MHz}\right)$ Spectrums of $((3 S, 5 S, 8 R, E)-3-(\mathrm{benzyl}$ oxy)-10-((3a $R, 5 R, 6 R, 6 \mathrm{a} R)-6$-(but-3-enyl)-2,2-dimethyltetrahydrofuro[2,3-d][1,3]dioxol-5-yl)-8-methoxy5,9-dimethyldeca-1,9-dien-6-yn-5-yloxy)trimethylsilane (19)

1H AMX500

SRK 4101_NaH/Me
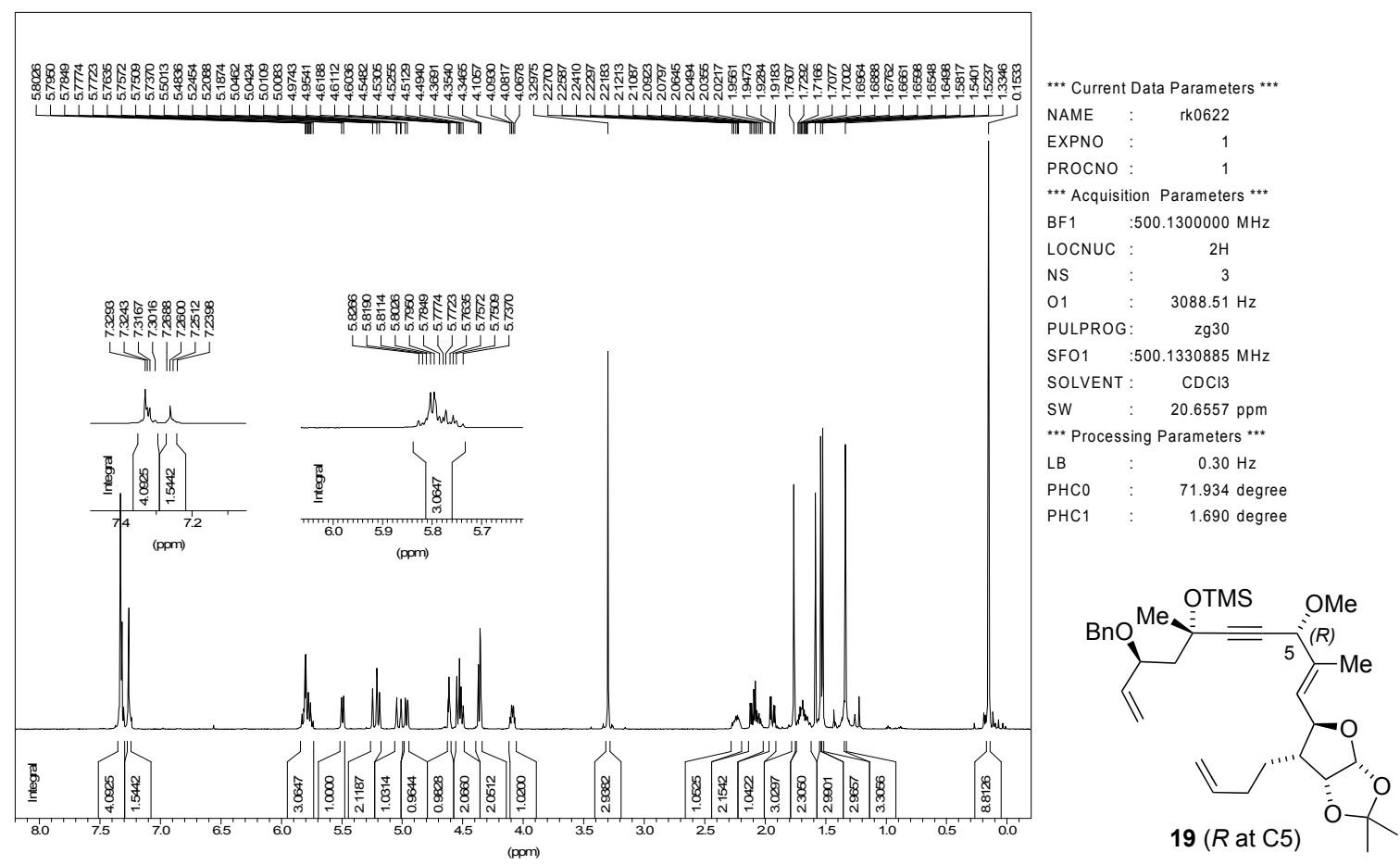

13C AMX500

SRK 4101_NaH/Mel
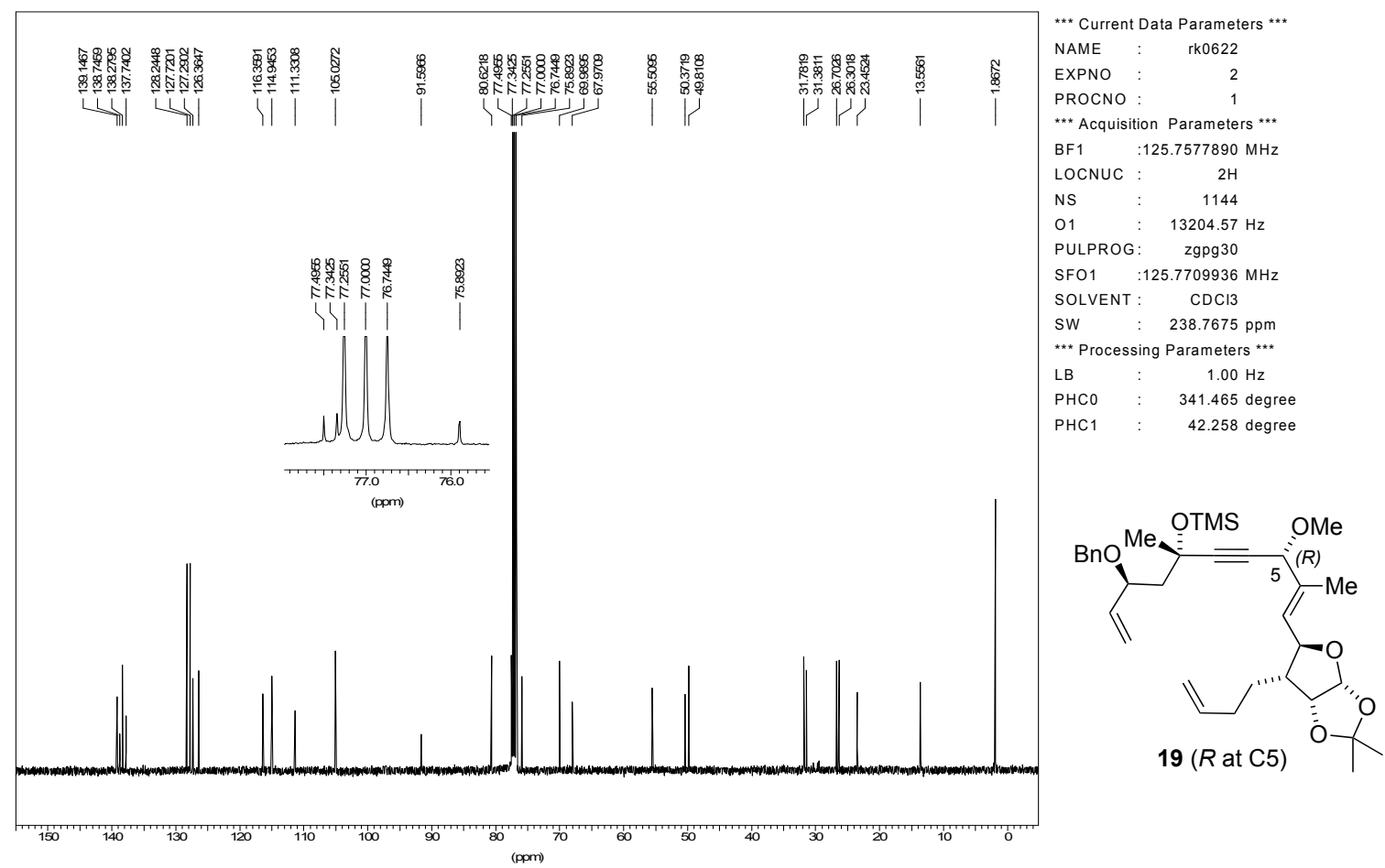
${ }^{1} \mathrm{H}$ NMR (500MHz, $\left.\mathrm{CDCl}_{3}\right)$ Spectrum of $(4 S, 7 \mathrm{R})-4-((S)-2-(b e n z y l o x y) b u t-3-e n y l)-7-((E)-1-((3 \mathrm{a} R, 5 R, 6 R$, 6aR)-6-(but-3-enyl)-2,2-dimethyltetrahydrofuro[2,3- $d][1,3]$ dioxol-5-yl)prop-1-en-2-yl)-9,9-diethyl-2,2,4trimethyl-3,8-dioxa-2,9-disilaundec-5-yne (20a)

$1 \mathrm{H}$ AMX500

4121_TES Of top

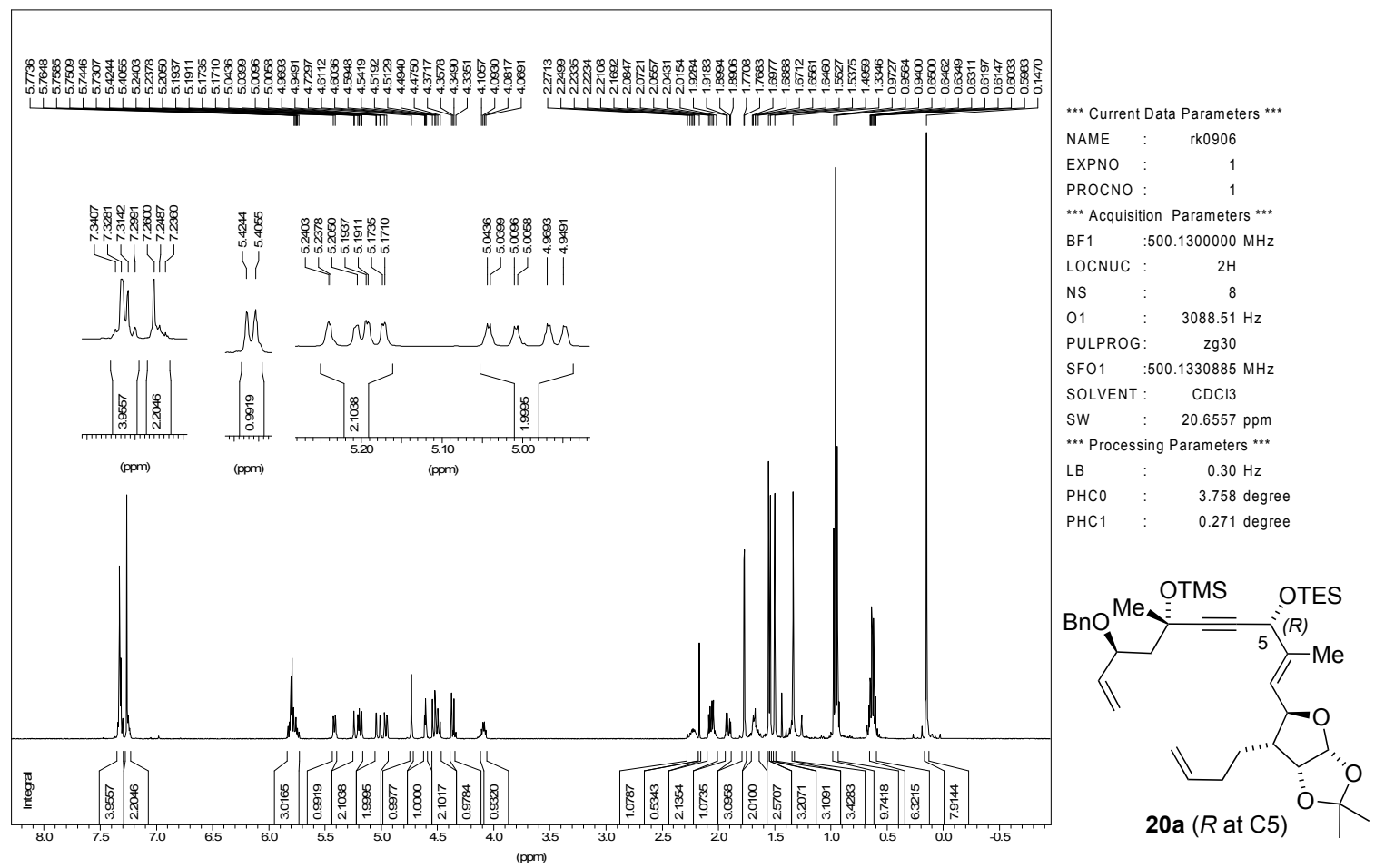

${ }^{1} \mathrm{H}$ NMR (500MHz, $\left.\mathrm{CDCl}_{3}\right)$ and ${ }^{13} \mathrm{C}\{1 \mathrm{H}\}$ NMR $\left(\mathrm{CDCl}_{3}, 125 \mathrm{MHz}\right)$ Spectrums of $(4 S, 7 S)-4-((S)-2-$ (benzyloxy)but-3-enyl)-7-((E)-1-((3aR,5R,6R,6aR)-6-(but-3-enyl)-2,2-dimethyltetrahydro furo[2,3- $d][1,3] \mathrm{di}$ oxol-5-yl)prop-1-en-2-yl)-9,9-diethyl-2,2,4-trimethyl-3,8-dioxa-2,9-disilaundec-5-yne (20b)

1H AMX500

4119_TES protection of btm diasteromer

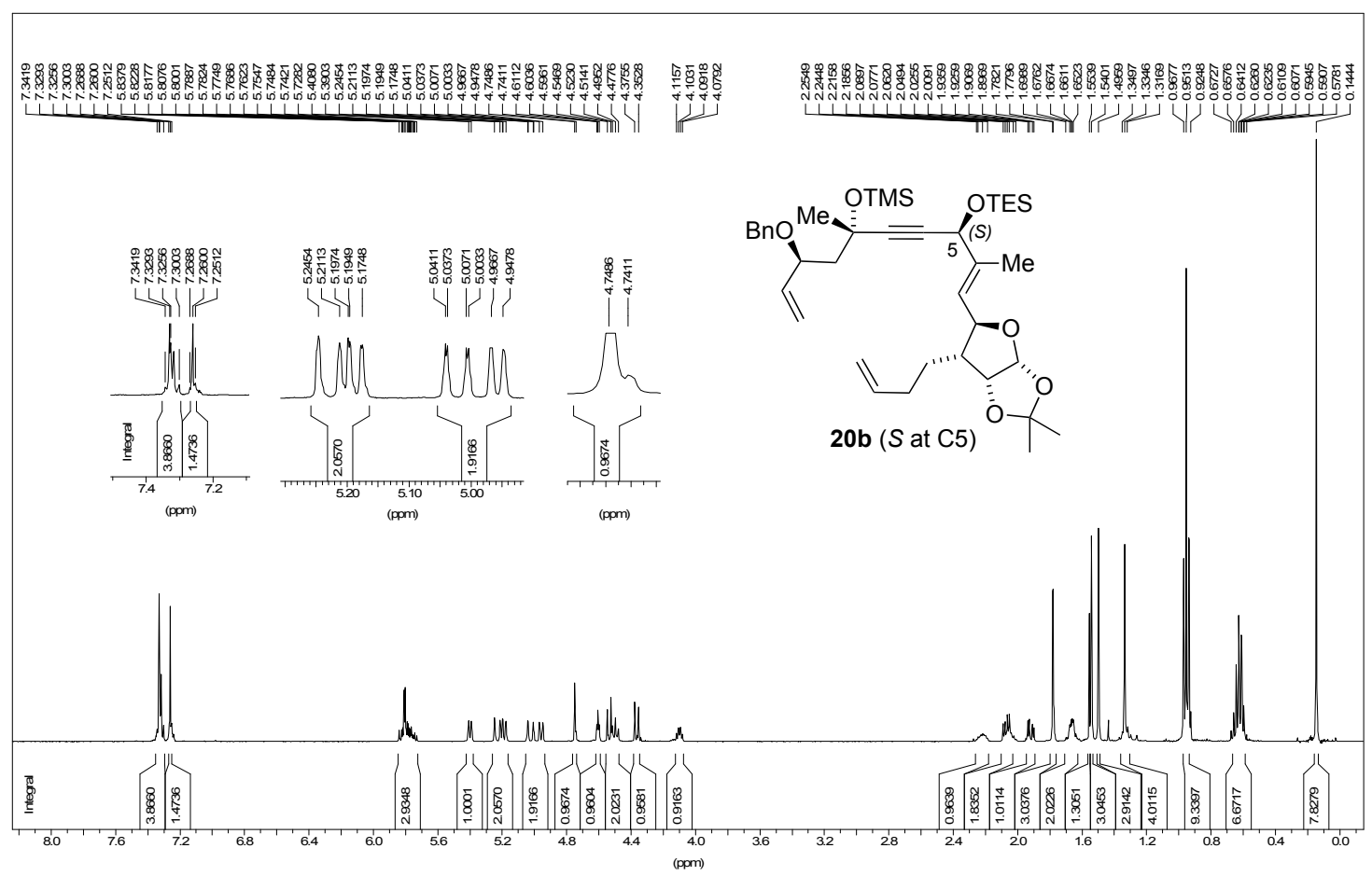



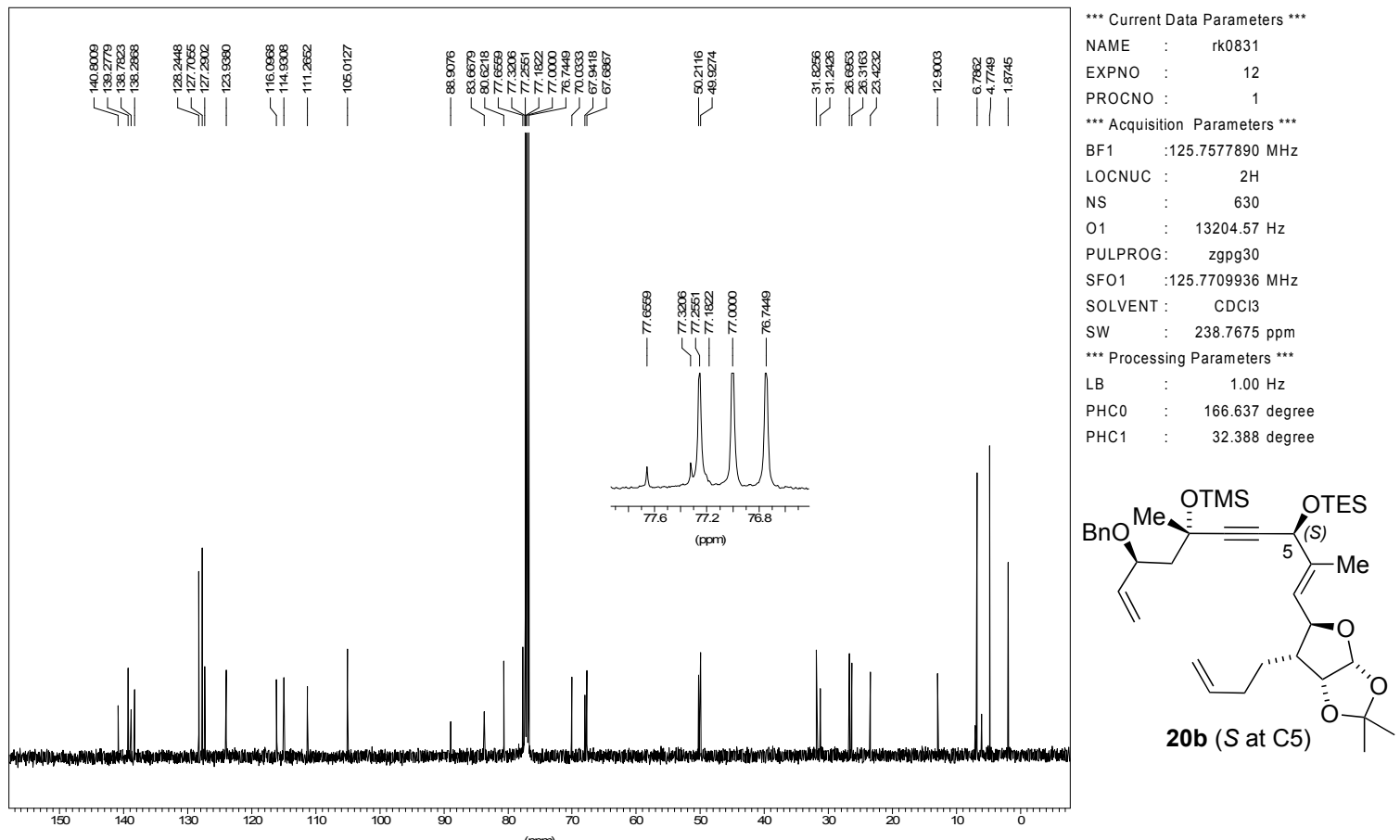

${ }^{1} \mathrm{H}$ NMR (500MHz, $\left.\mathrm{CDCl}_{3}\right)$ and ${ }^{13} \mathrm{C}\{1 \mathrm{H}\}$ NMR $\left(\mathrm{CDCl}_{3}, 125 \mathrm{MHz}\right)$ Spectrums of $(6 S, 8 S, E)-8$-(benzyloxy)-1((3aR,5R,6R,6aR)-6-(but-3-en-1-yl)-2,2-dimethyltetrahydrofuro[2,3- $d][1,3]$ dioxol-5-yl)-2,6-dimethyl-6((trimethylsilyl)oxy)deca-1,9-dien-4-yn-3-one (21)

1 H AMX500

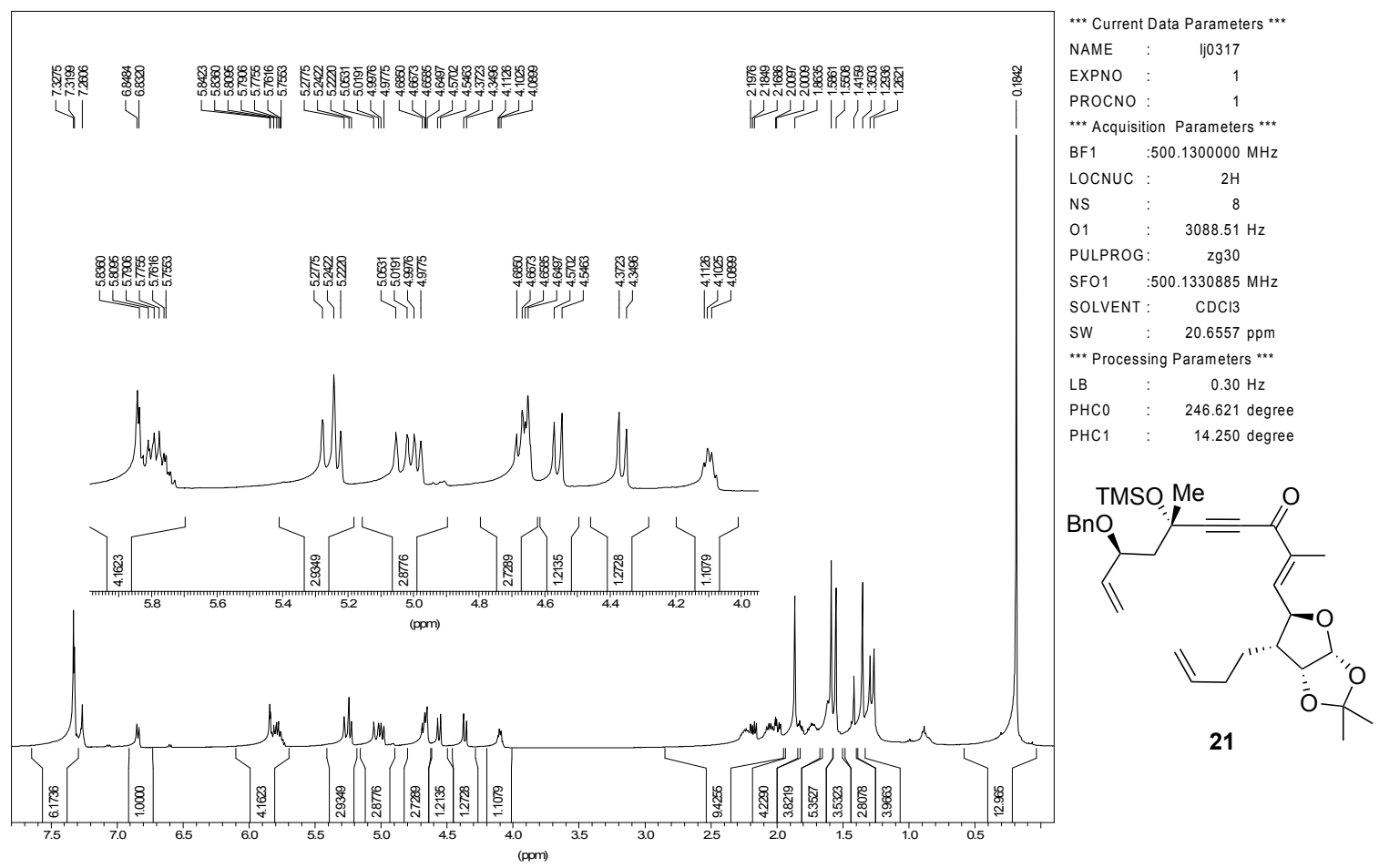




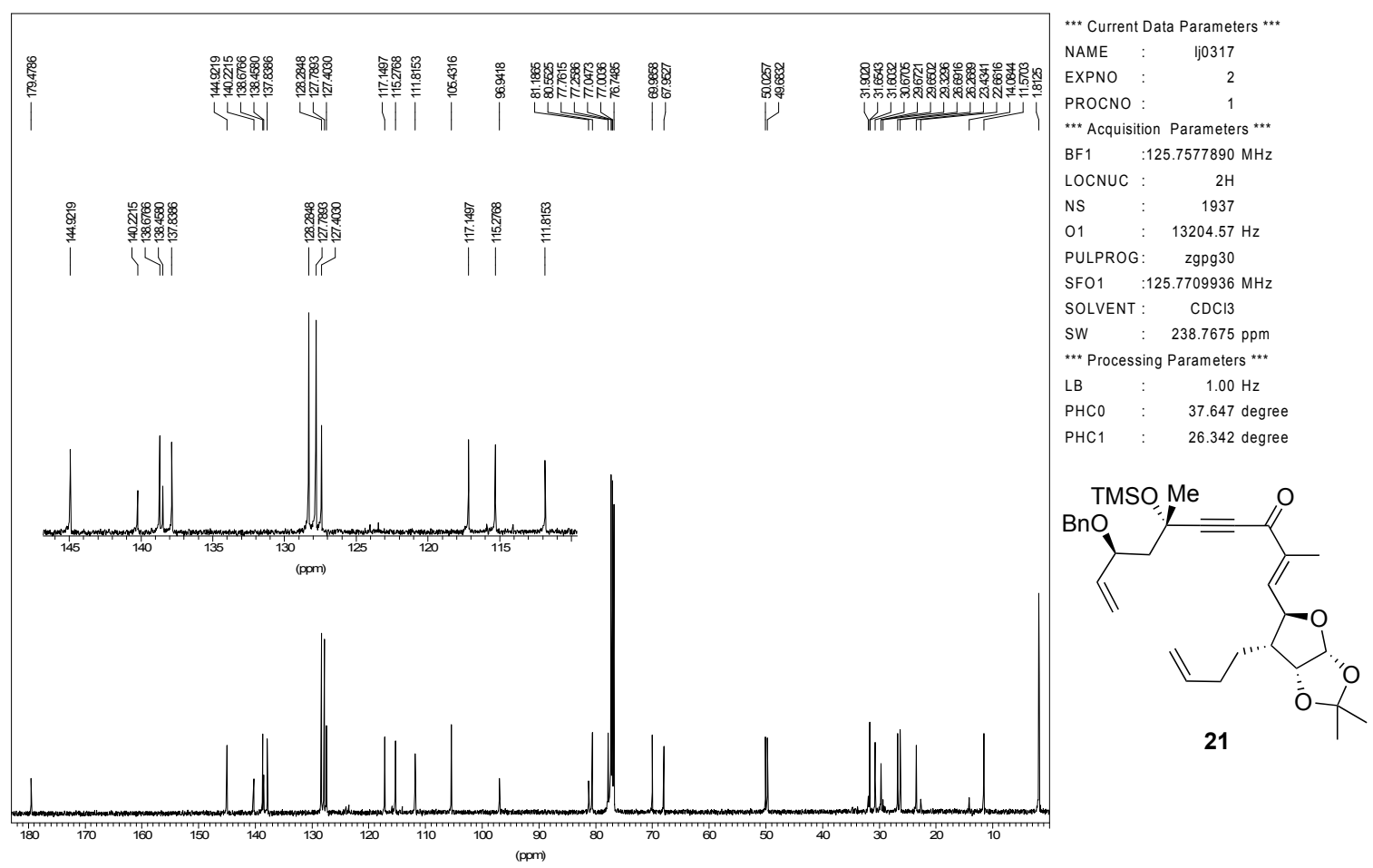

${ }^{1} \mathrm{H}$ NMR (500MHz, $\left.\mathrm{CDCl}_{3}\right)$ and ${ }^{13} \mathrm{C}\{1 \mathrm{H}\} \mathrm{NMR}\left(\mathrm{CDCl}_{3}, 125 \mathrm{MHz}\right.$ and DEPT) Spectrums of 22

1H AMX500

GH-II_new spot (rk0728_expt 1)

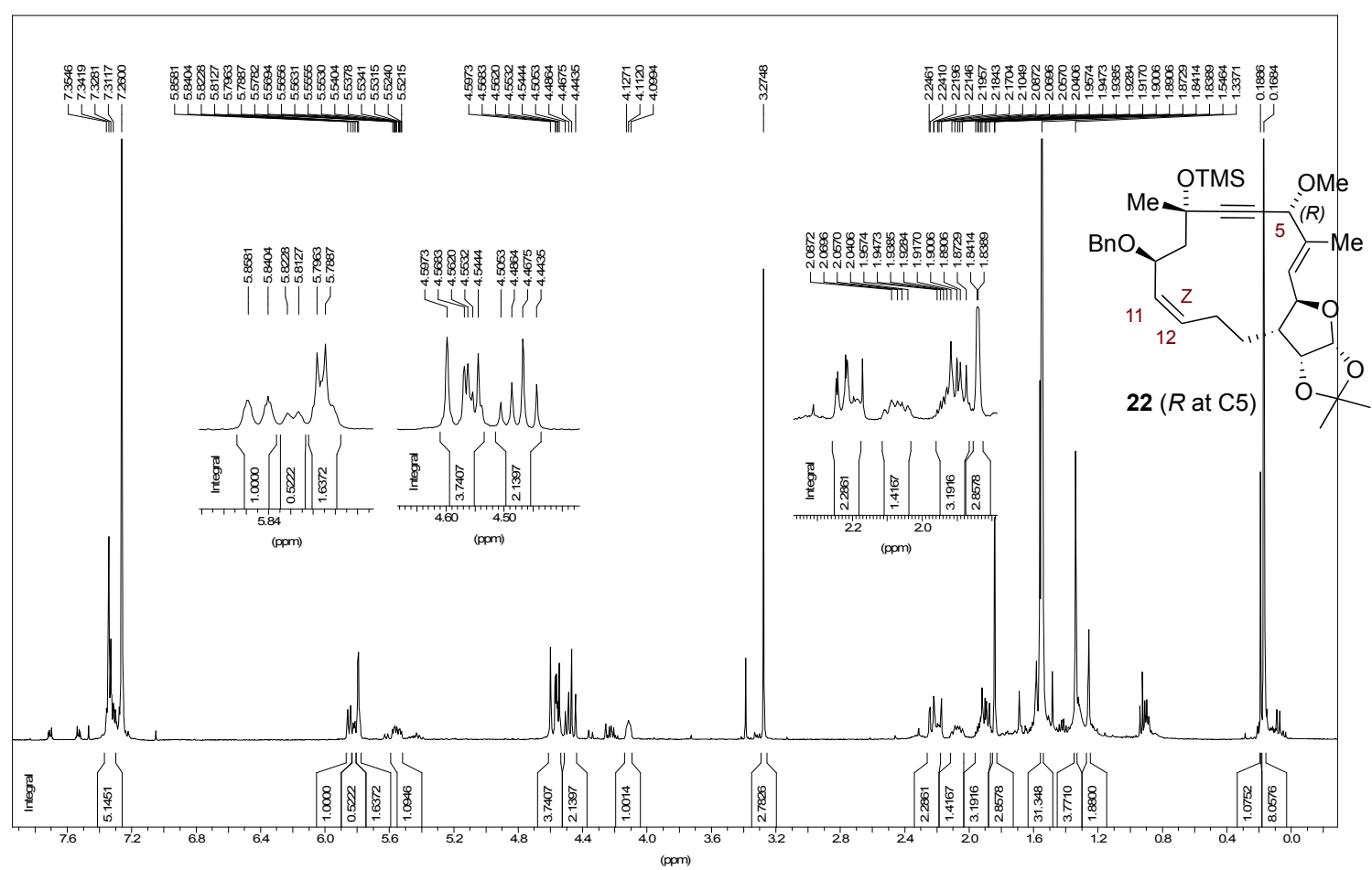




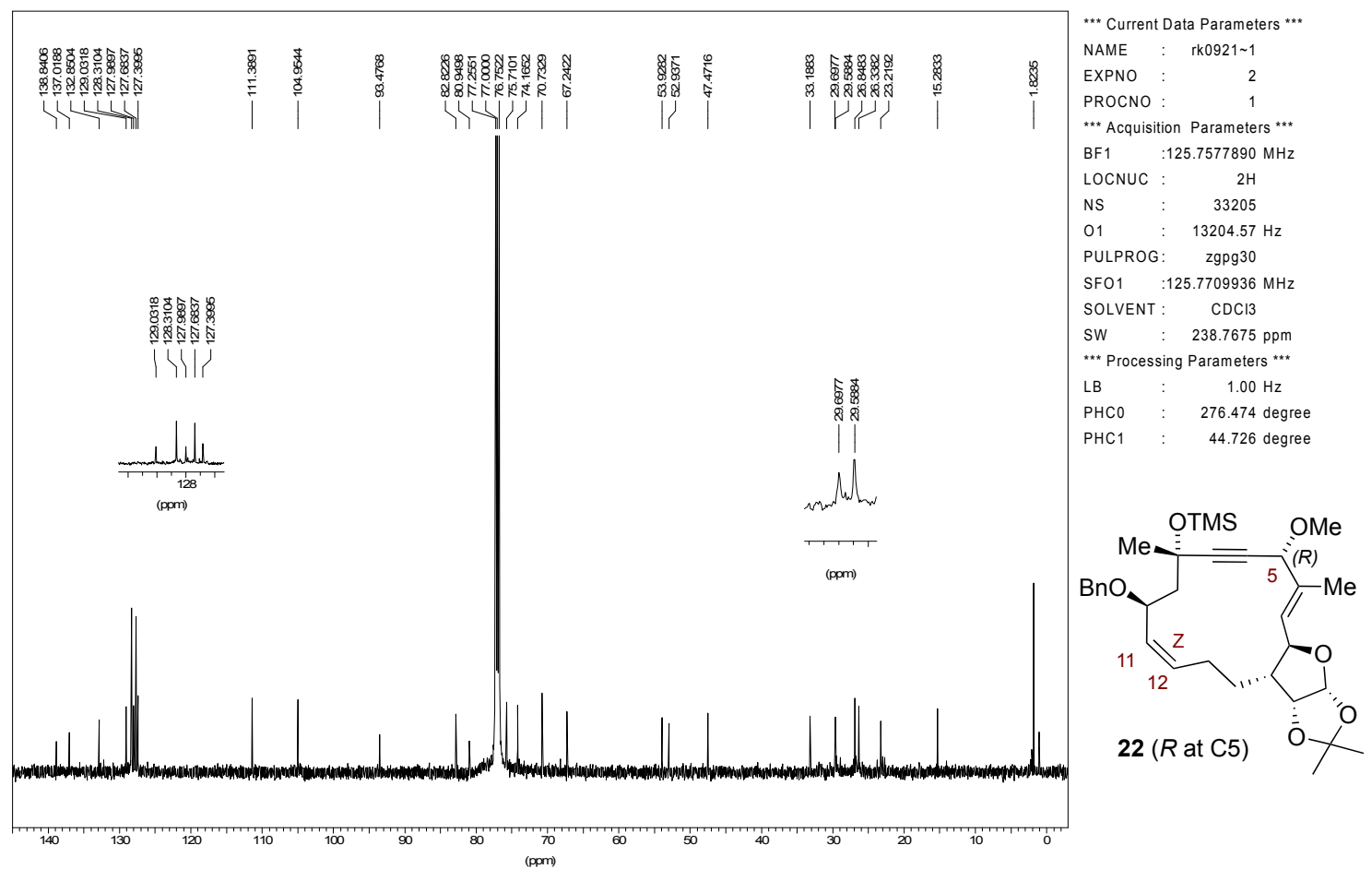

DEPT135 AMX500

RCM product of Biels (rk0921_2 nights scanned exp 3)

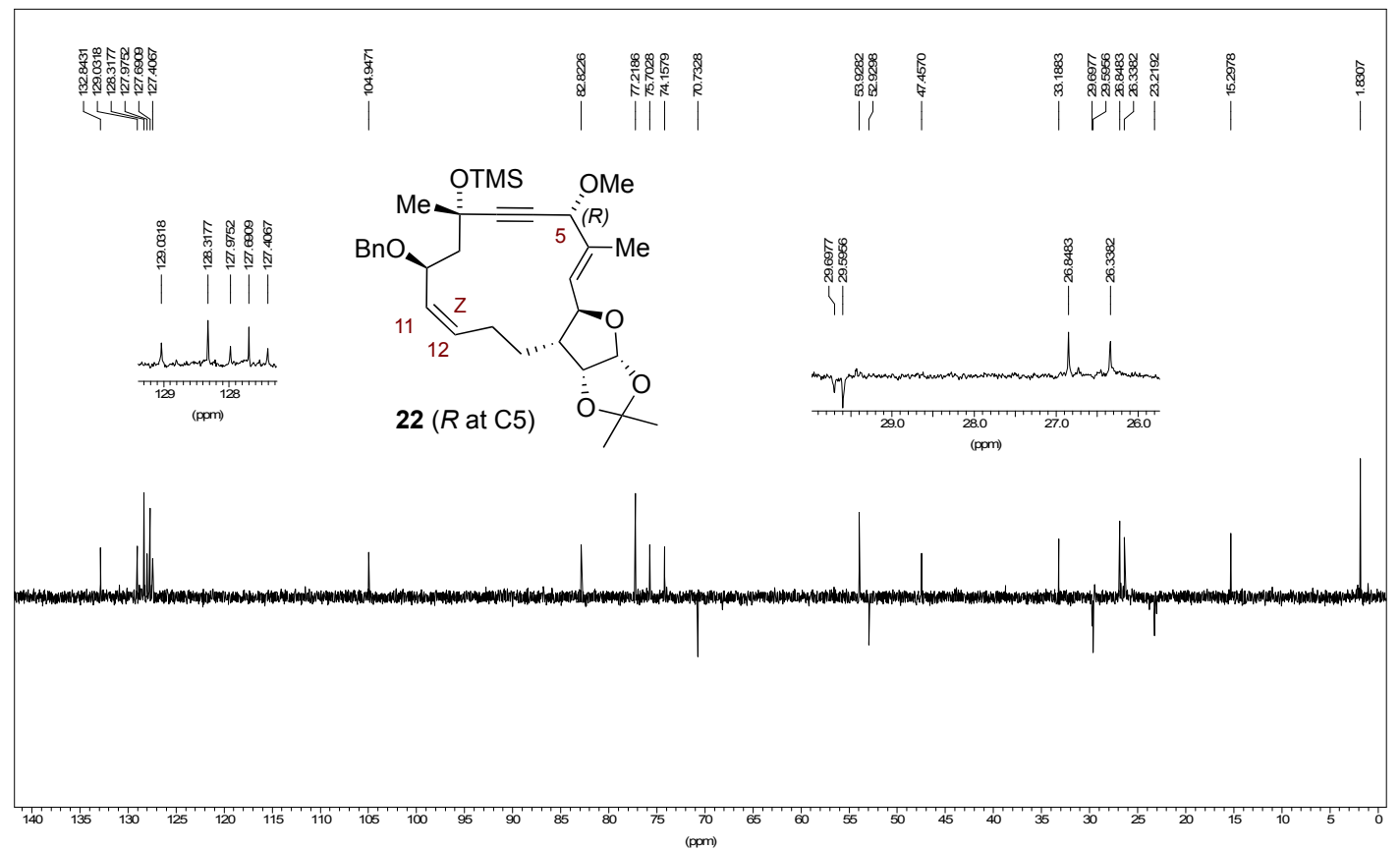


COSY Spectrum (22)
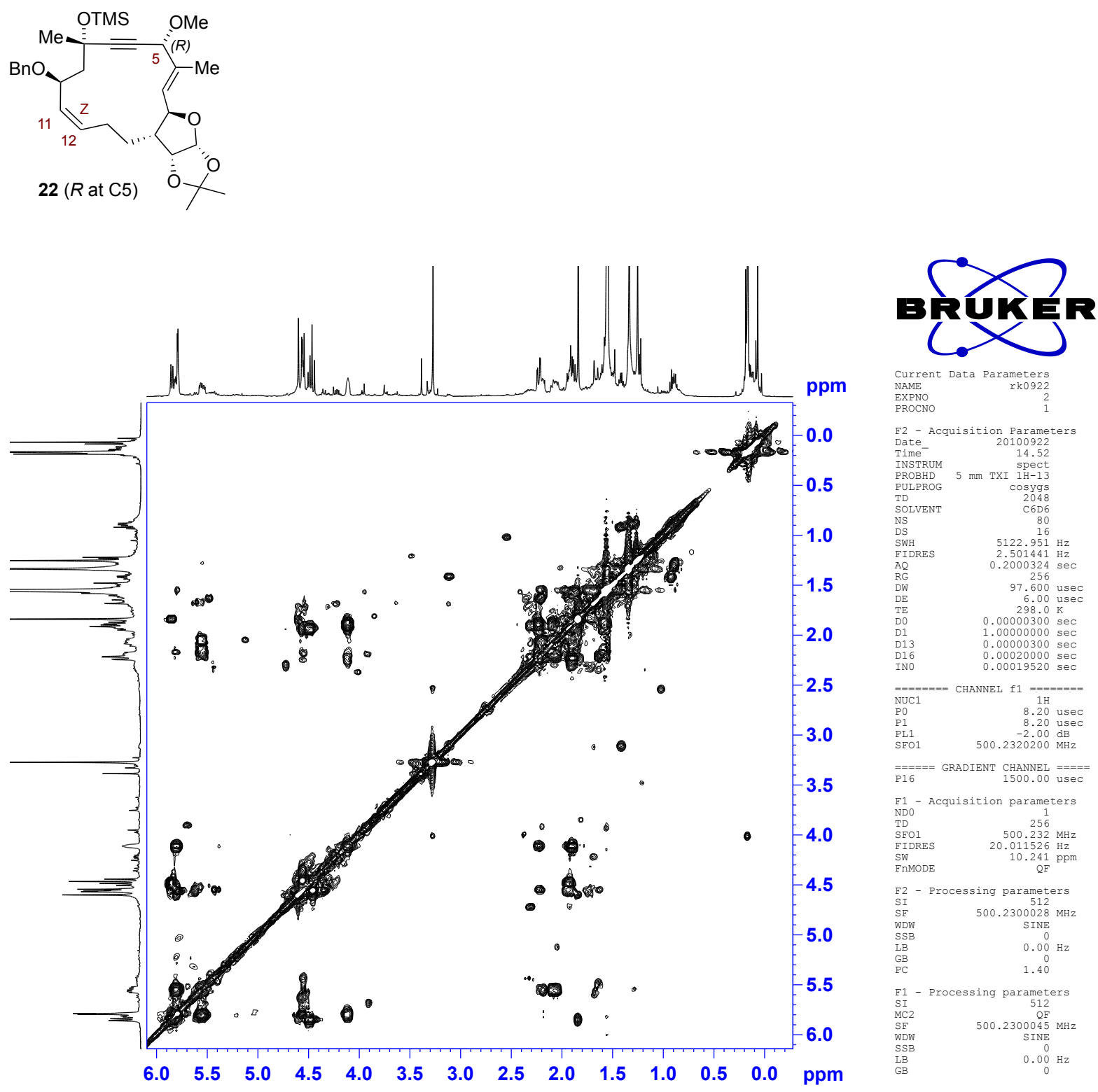
HMQC Spectrum of 22
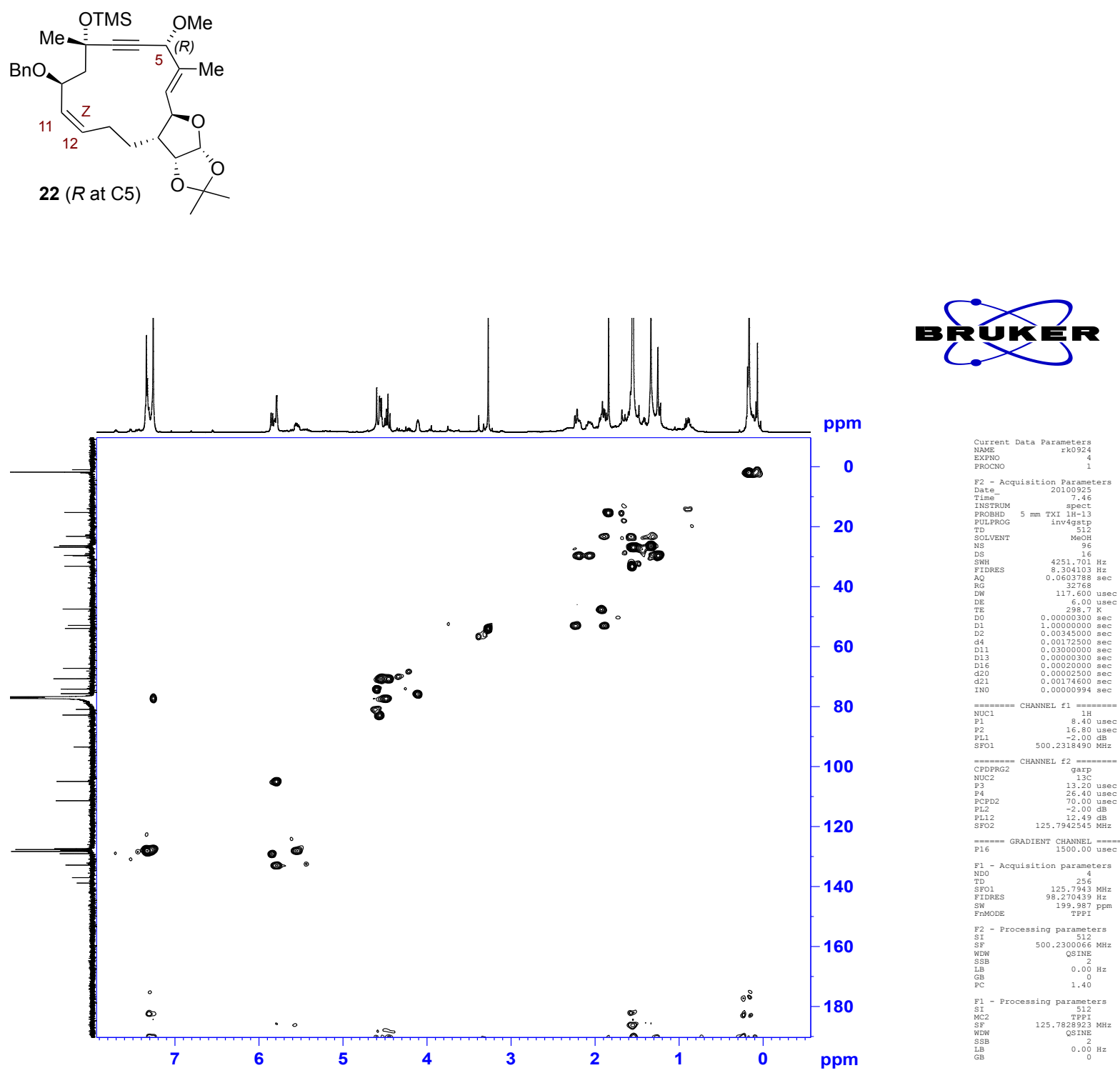


\section{NOESY (22)}
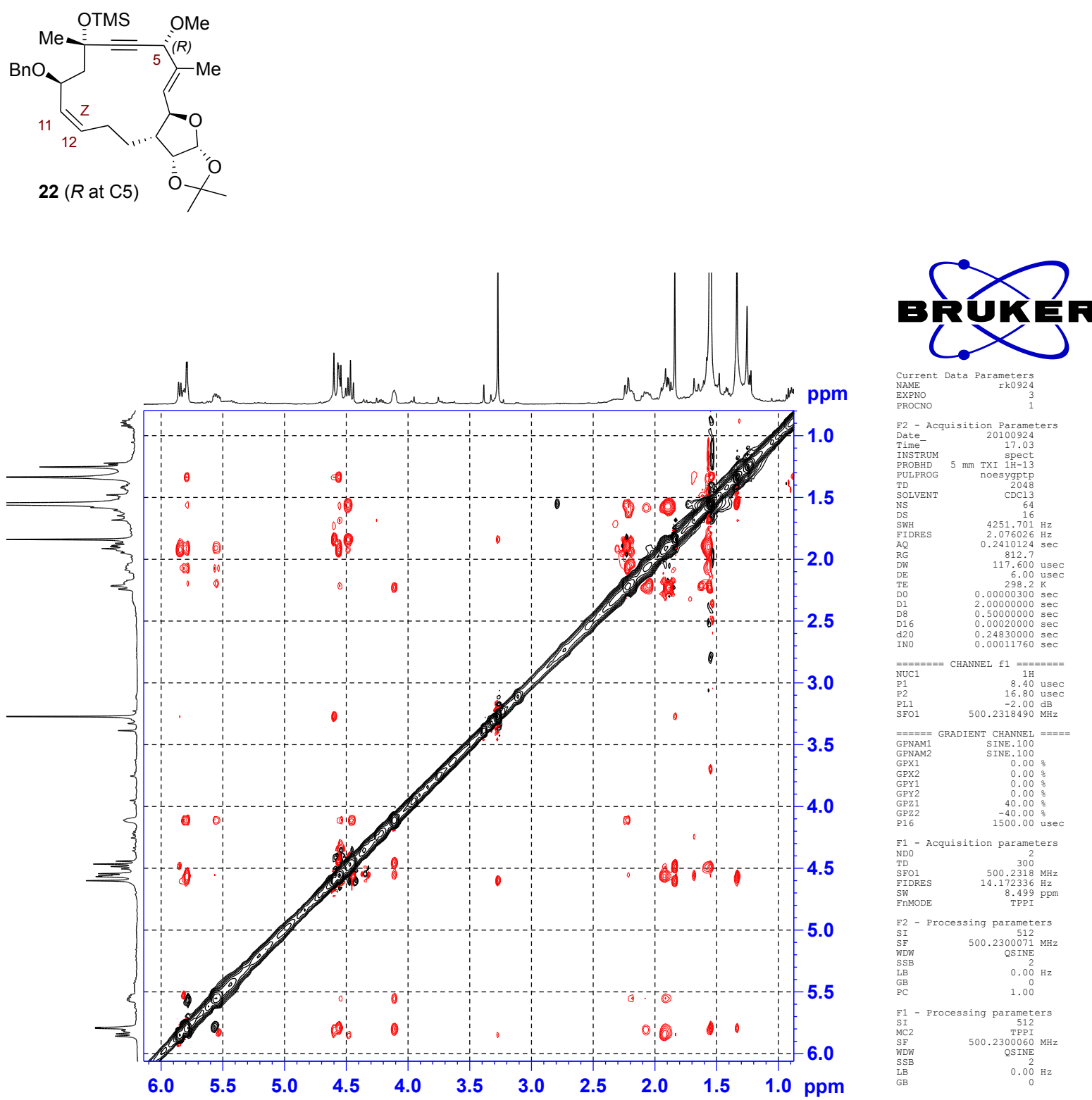
${ }^{1} \mathrm{H}$ NMR (500MHz, $\left.\mathrm{CDCl}_{3}\right)$ and ${ }^{13} \mathrm{C}\{1 \mathrm{H}\}$ NMR $\left(\mathrm{CDCl}_{3}, 125 \mathrm{MHz}\right)$ Spectrums of $(2 R, 4 S, 6 S)$-4-ethynyl-2phenyl-6-vinyl-1,3-dioxane (27h):
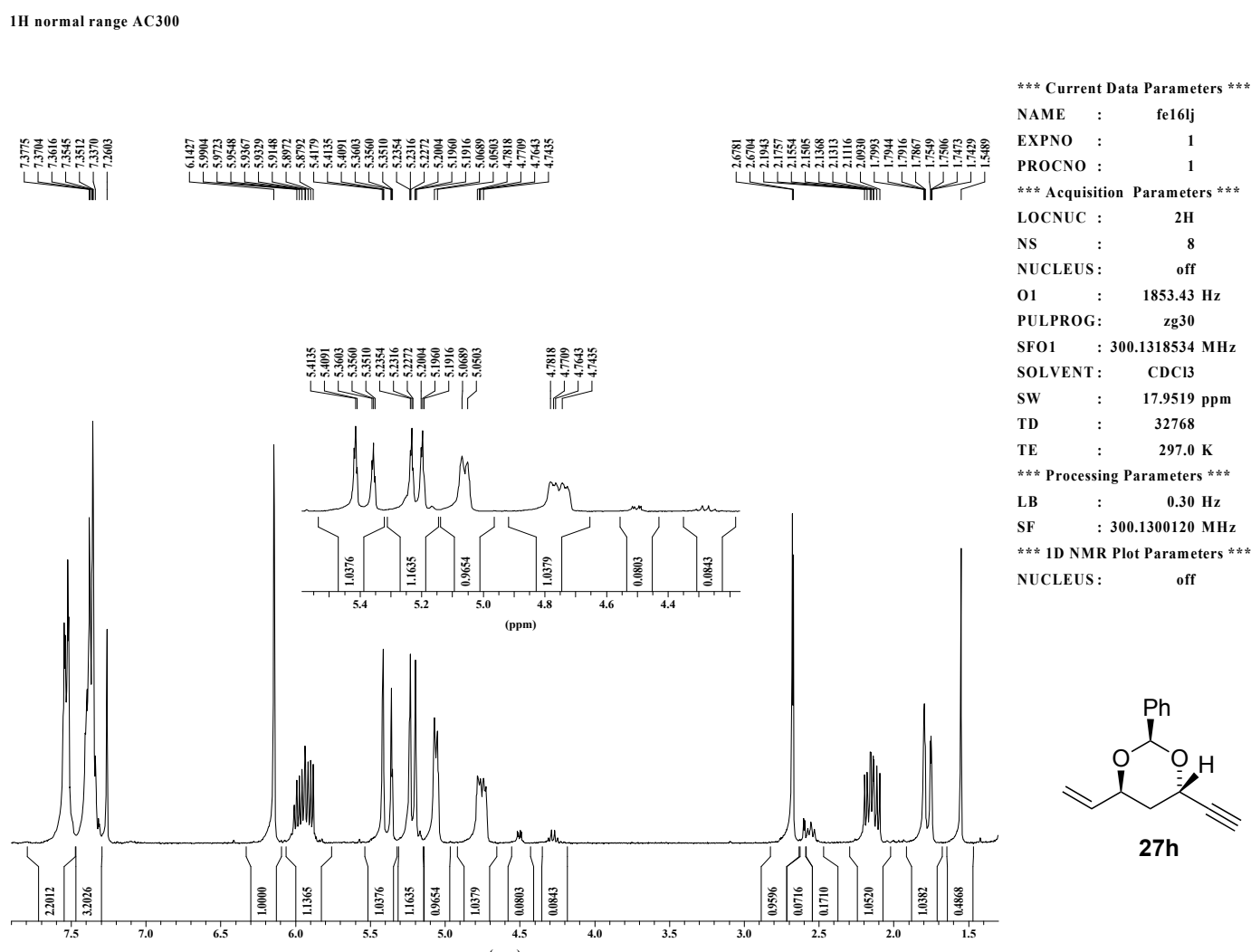

13C Standard AC300

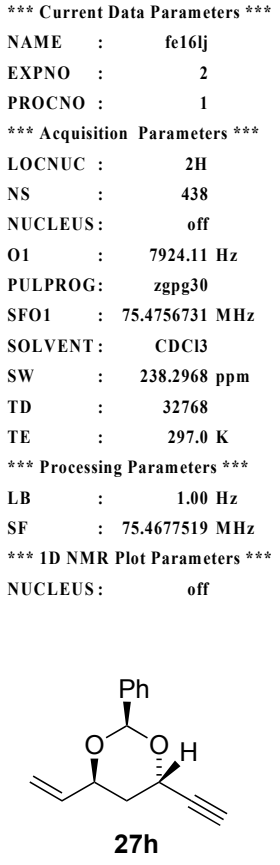

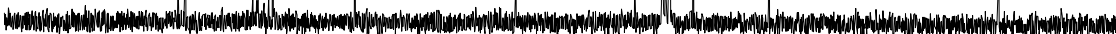

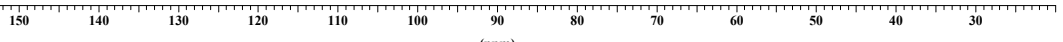


${ }^{1} \mathrm{H}$ NMR (500MHz, $\left.\mathrm{CDCl}_{3}\right)$ and ${ }^{13} \mathrm{C}\{1 \mathrm{H}\}$ NMR $\left(\mathrm{CDCl}_{3}, 125 \mathrm{MHz}\right)$ Spectrums of $(E)-1-((3 \mathrm{a} R, 5 R, 6 R, 6 \mathrm{a} R)-6-$ (but-3-en-1-yl)-2,2-dimethyltetrahydrofuro[2,3- $d][1,3]$ dioxol-5-yl)-2-methyl-5-((2R,4S,6S)-2-phenyl-6vinyl-1,3-dioxan-4-yl)pent-1-en-4-yn-3-ol (28h)

1 H AMX500

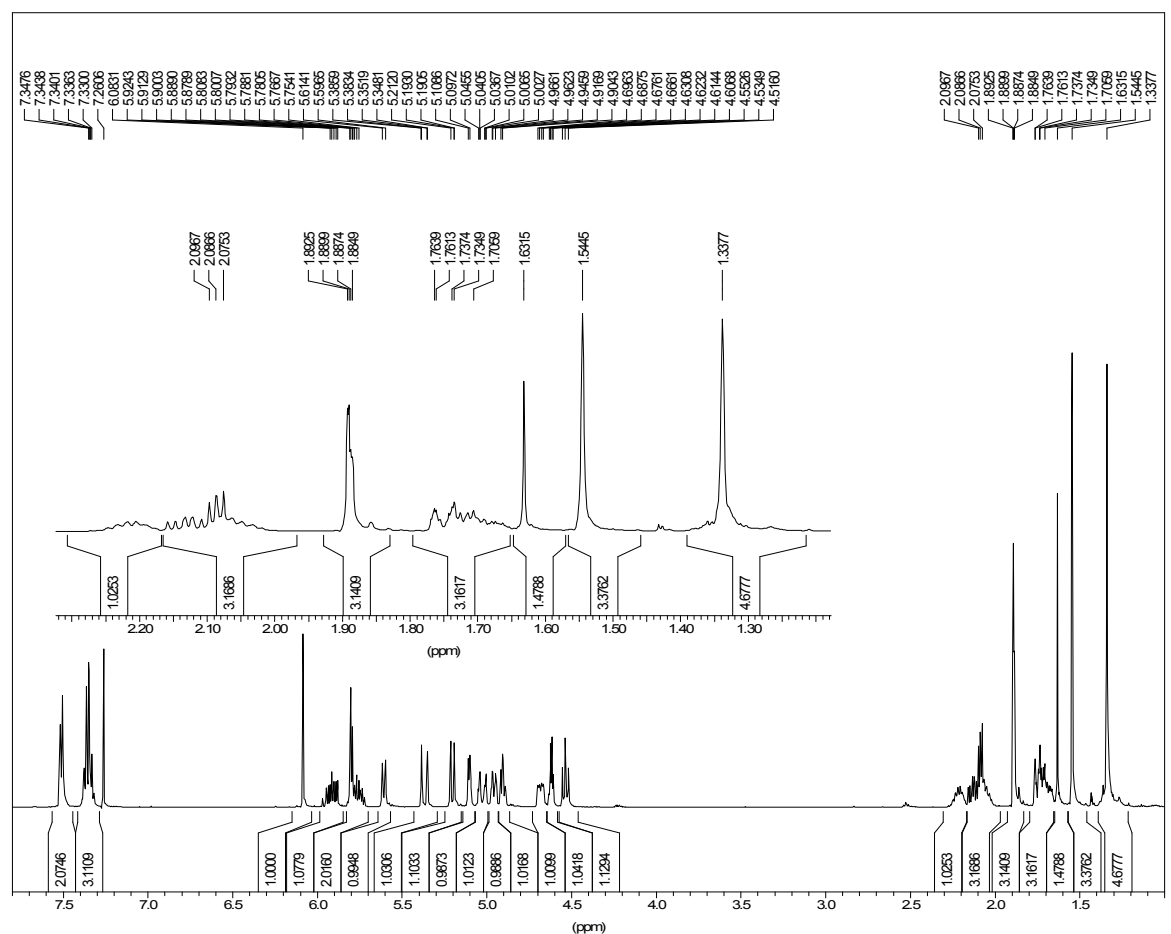

*** Current Data Parameters **

NAME : $\quad$ lj0226

EXPNO : $\quad 1$

PROCNO: $\quad 1$

*** Acquisition Parameters ***

BF1 : $500.1300000 \mathrm{MHz}$

LOCNUC : $\quad 2 \mathrm{H}$

$\begin{array}{llc}\text { NS } & : & 8 \\ 01 & : & 3088.51 \mathrm{~Hz}\end{array}$

PULPROG: $\quad \mathrm{zg} 30$

SF01 : $500.1330885 \mathrm{MHz}$

SOLVENT : $\quad \mathrm{CDCl} 3$

SW : $\quad 20.6557 \mathrm{ppm}$

*** Processing Parameters ***

LB : $\quad 0.30 \mathrm{~Hz}$

PHC0 : 158.987 degre

PHC1 : 0.009 degree

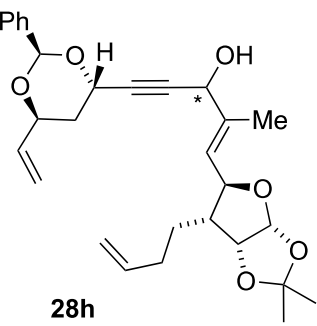

13C AMX500

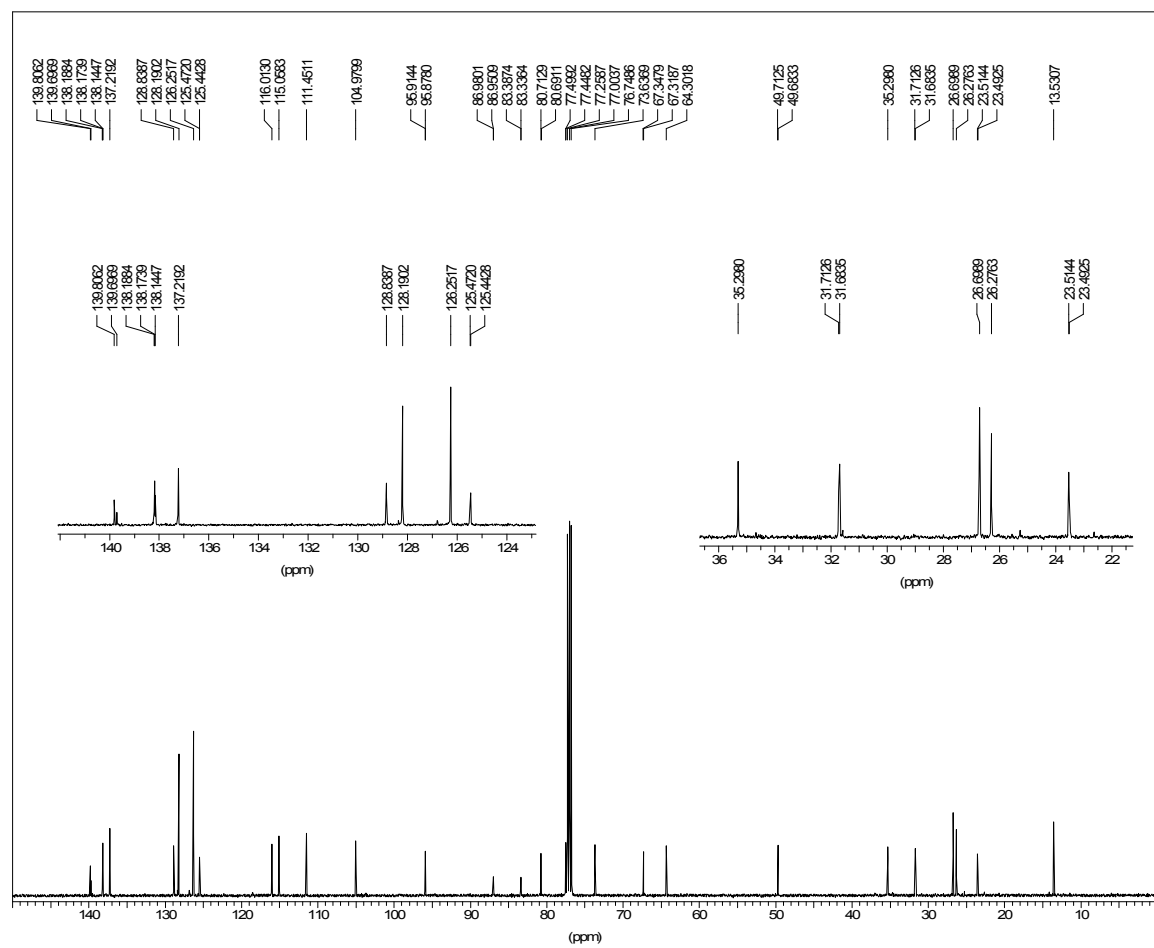

"** Current Data Parameters ***

NAME : $\quad$ lj0226

EXPNO : 2

EROCNO :

*** Acquisition Parameters ***

BF1 : $125.7577890 \mathrm{MHz}$

LOCNUC : $\quad 2 \mathrm{H}$

NS : 1609

$\begin{array}{llc}\text { NS } & : & 1609 \\ 01 & : & 13204.57 \mathrm{~Hz}\end{array}$

PULPROG: $\quad$ zgpg30

SFO1 : $125.7709936 \mathrm{MHz}$

SOLVENT : $\quad \mathrm{CDCl} 3$

SW : $238.7675 \mathrm{ppm}$

*** Processing Parameters **

LB : $\quad 1.00 \mathrm{~Hz}$

PHCO : $\quad 300.313$ degre

PHC1 : $\quad 39.027$ degree

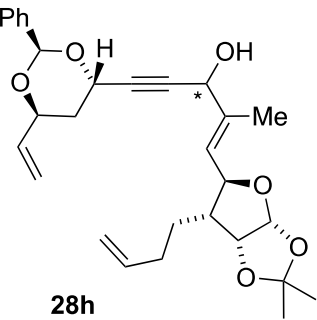


${ }^{1} \mathrm{H}$ NMR (500MHz, $\left.\mathrm{CDCl}_{3}\right)$ and ${ }^{13} \mathrm{C}\{1 \mathrm{H}\}$ NMR $\left(\mathrm{CDCl}_{3}, 125 \mathrm{MHz}\right)$ Spectrums of $(E)-1-((3 \mathrm{a} R, 5 R, 6 R, 6 \mathrm{a} R)-6-$ (but-3-en-1-yl)-2,2-dimethyltetrahydrofuro[2,3- $d][1,3]$ dioxol-5-yl)-2-methyl-5-((2R,4S,6S)-2-phenyl-6vinyl-1,3-dioxan-4-yl)pent-1-en-4-yn-3-one (31)

$1 \mathrm{H}$ AMX500

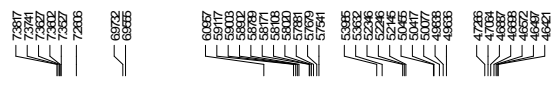

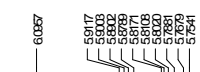
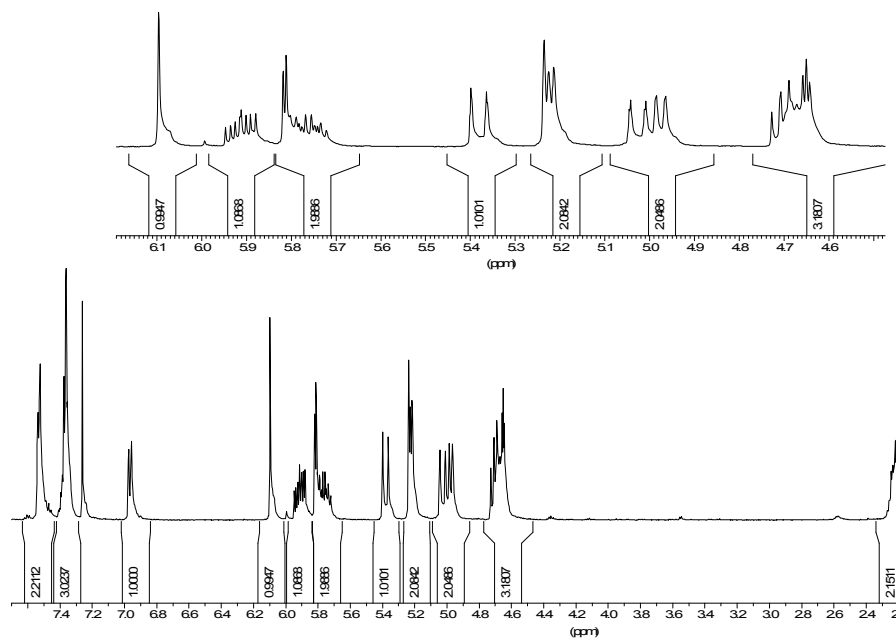

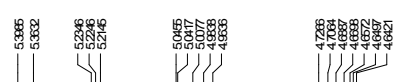
53

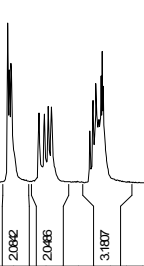

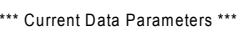

NAME : test1

EXPNO :

PROCNO: 1

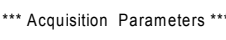

NUCLEUS: NUC?

$01: \quad 4250.00 \mathrm{~Hz}$

PULPROG: TOSS.PC

SFO1 : $75.4842500 \mathrm{MHz}$

SOLVENT : Solvent?

SW : $662.4096 \mathrm{ppm}$

TD : 2048

${ }^{\star \star \star}$ Processing Parameters ${ }^{\star \star \star}$

*** 1D NMR Plot $\quad 0.00 \mathrm{~Hz}$

NUCLEUS: ?

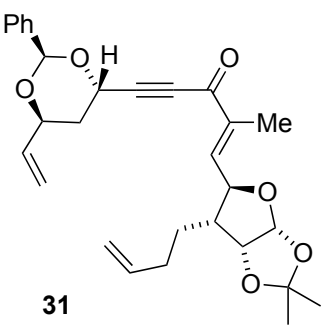

13C AMX500

*** Current Data Parameters ***

NAME : test1

EXPNO : 1

PROCNO : 1

*** Acquisition Parameters ***

NUCLEUS: NUC?

Nuc?

$01: \quad 4250.00$

PULPROG: TOSS.PC

SFO1 : $75.4842500 \mathrm{MHz}$

SOLVENT : Solvent?

SW : $662.4096 \mathrm{ppm}$

$\begin{array}{rrr}\text { SW } & : & 662.4096 \\ \text { TD } \quad: & 2048\end{array}$

*** Processing Parameters ***

*** 1D NMR Plot Parameters *

NUCLEUS: ?

31

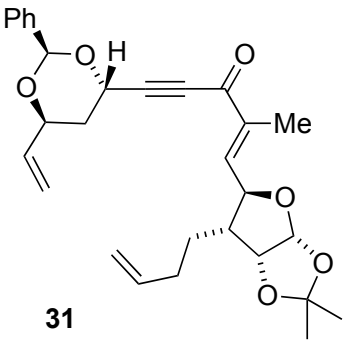


${ }^{1} \mathrm{H}$ NMR (500MHz, $\left.\mathrm{CDCl}_{3}\right)$ and ${ }^{13} \mathrm{C}\{1 \mathrm{H}\}$ NMR $\left(\mathrm{CDCl}_{3}, 125 \mathrm{MHz}\right)$ Spectrums of $(2 S, 4 S, 6 S)$-4-ethynyl-4methyl-2-phenyl-6-vinyl-1,3-dioxane (27)

$1 \mathrm{H} \mathrm{AMX} \times 500$

4018_1 (rk1110 exp 11)

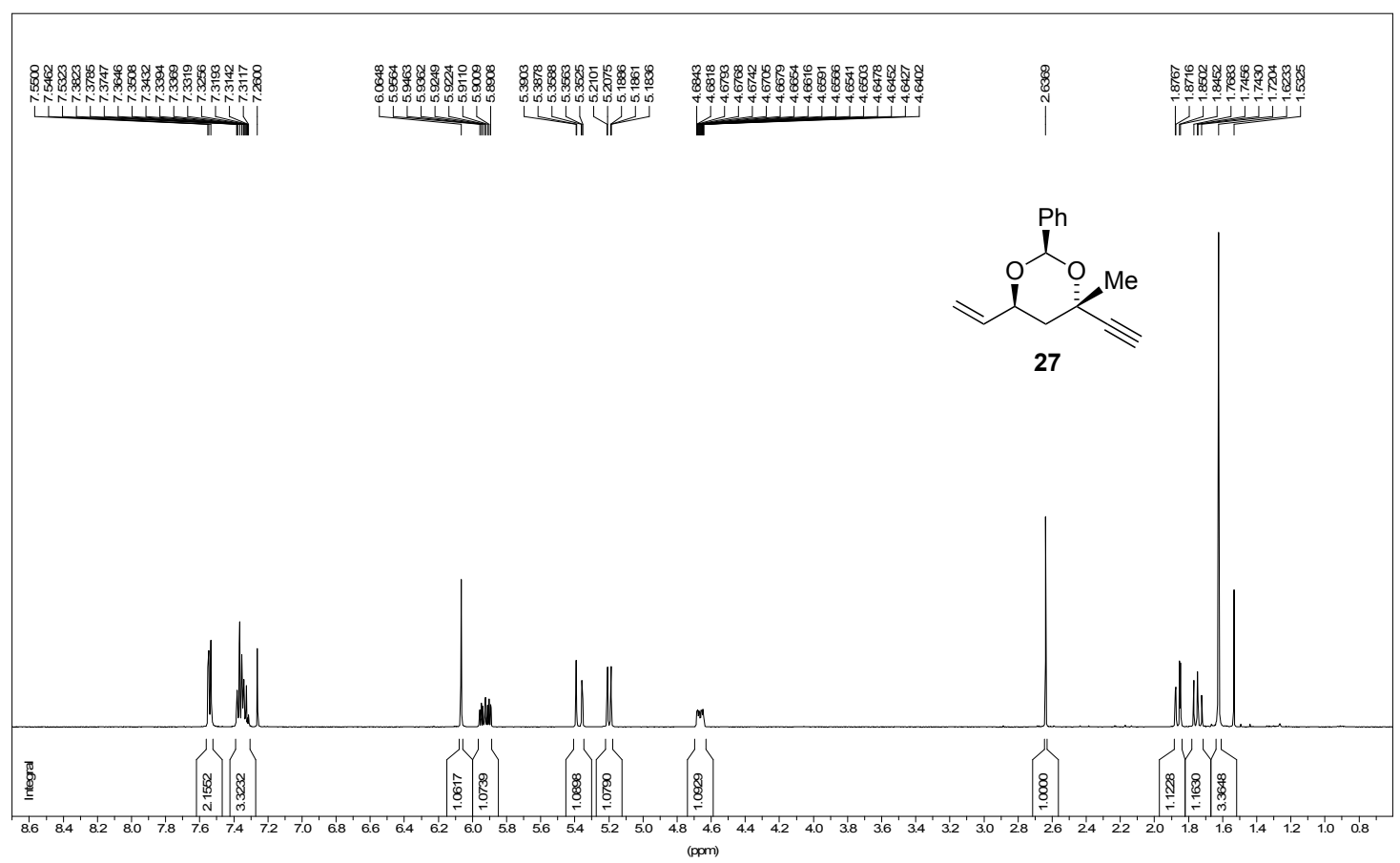

13C AMX500

4018_1 (rk1110 exp 12)

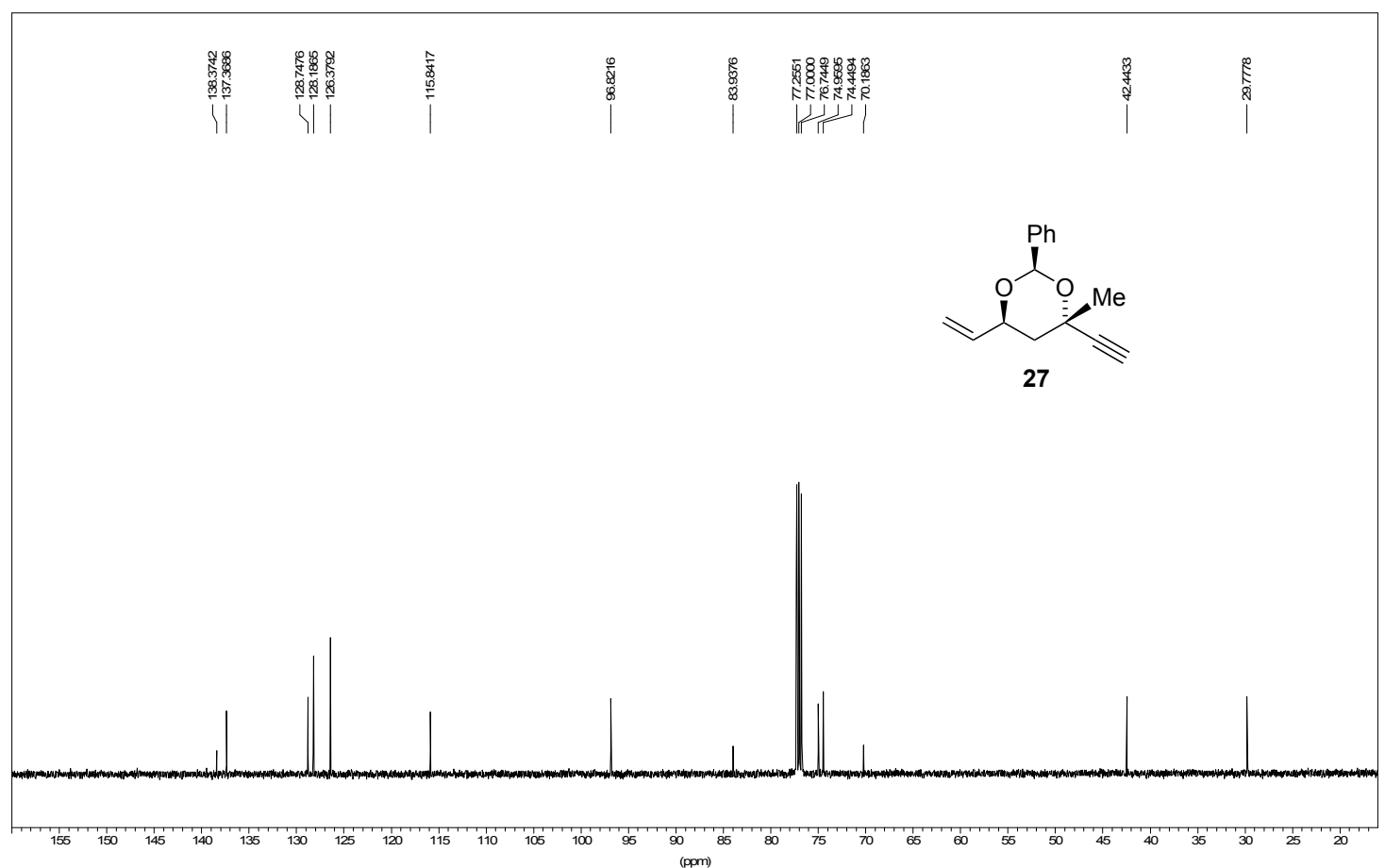


${ }^{1} \mathrm{H}$ NMR (500MHz, $\left.\mathrm{CDCl}_{3}\right)$ and ${ }^{13} \mathrm{C}\{1 \mathrm{H}\}$ NMR $\left(\mathrm{CDCl}_{3}, 125 \mathrm{MHz}\right)$ Spectrums of $(E)-1-((3 \mathrm{a} R, 5 R, 6 R, 6 \mathrm{a} R)-6-$ (but-3-en-1-yl)-2,2-dimethyltetrahydrofuro[2,3- $d][1,3]$ dioxol-5-yl)-2-methyl-5-((2R,4S,6S)-4-methyl-2-phe nyl-6-vinyl-1,3-dioxan-4-yl)pent-1-en -4-yn-3-ol (28)

$1 \mathrm{H}$ normal range $\mathrm{AC} 300$
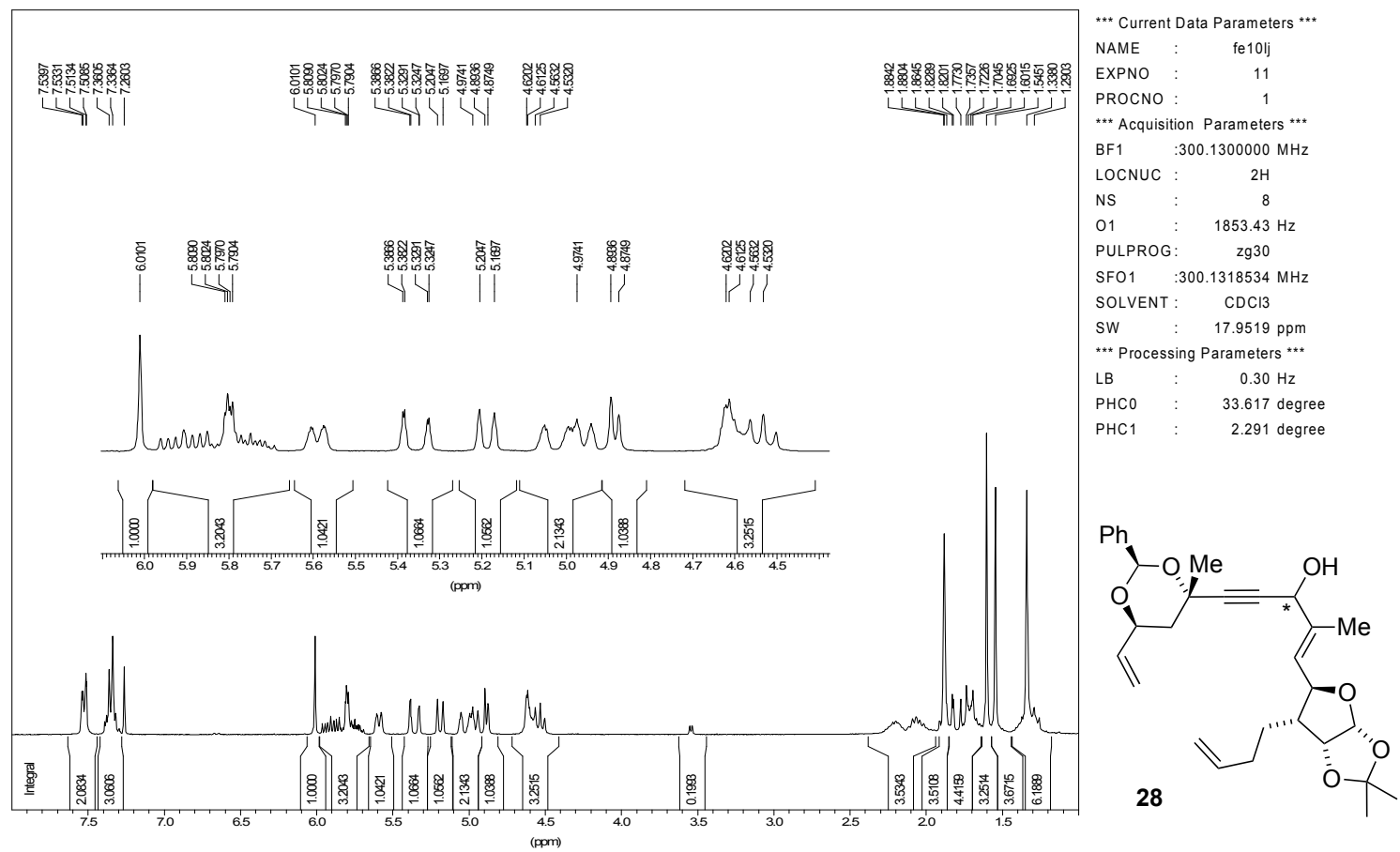

13C Standard AC300
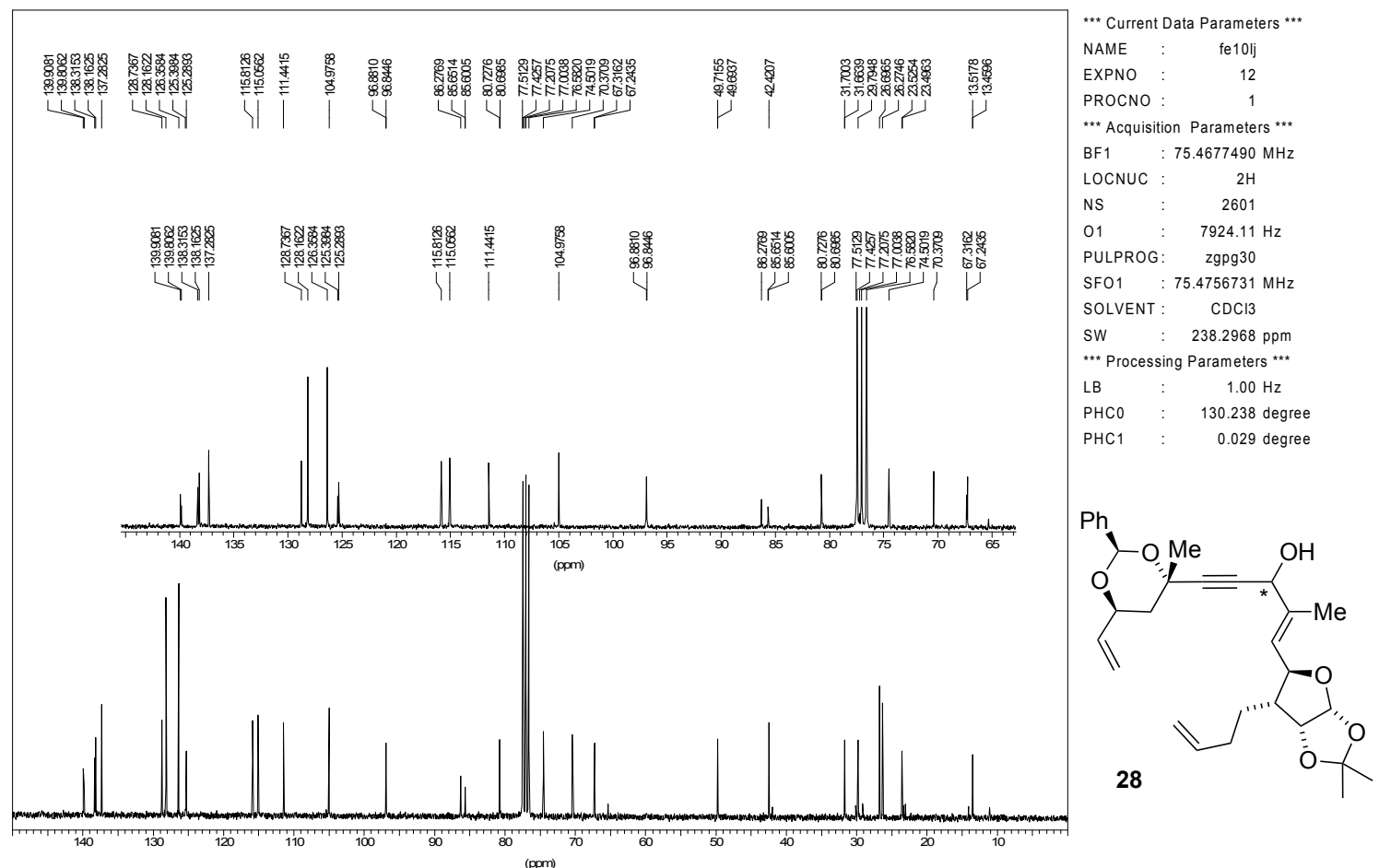
${ }^{1} \mathrm{H}$ NMR (500MHz, $\left.\mathrm{CDCl}_{3}\right)$ and ${ }^{13} \mathrm{C}\{1 \mathrm{H}\}$ NMR $\left(\mathrm{CDCl}_{3}, 125 \mathrm{MHz}\right)$ Spectrums of $(E)-1-((3 \mathrm{a} R, 5 R, 6 R, 6 \mathrm{a} R)-6-$ (but-3-en-1-yl)-2,2-dimethyltetrahydrofuro[2,3- $d][1,3]$ dioxol-5-yl)-2-methyl-5-((2R,4S,6S)-4-methyl-2-phe nyl-6-vinyl-1,3-dioxan-4-yl)pent-1-en -4-yn-3-one (29)

1 H AMX500
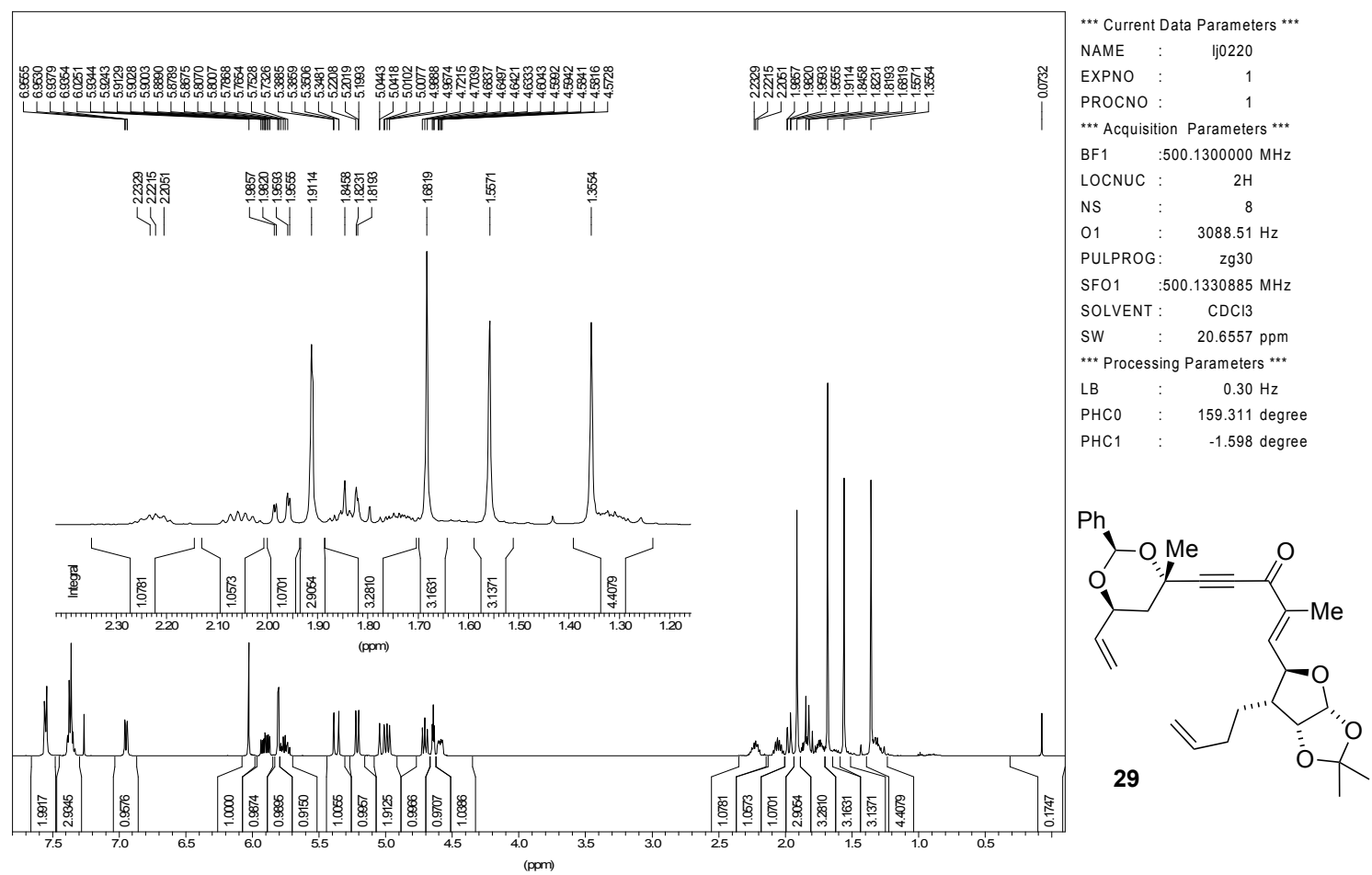

$13 \mathrm{C}$ AMX500
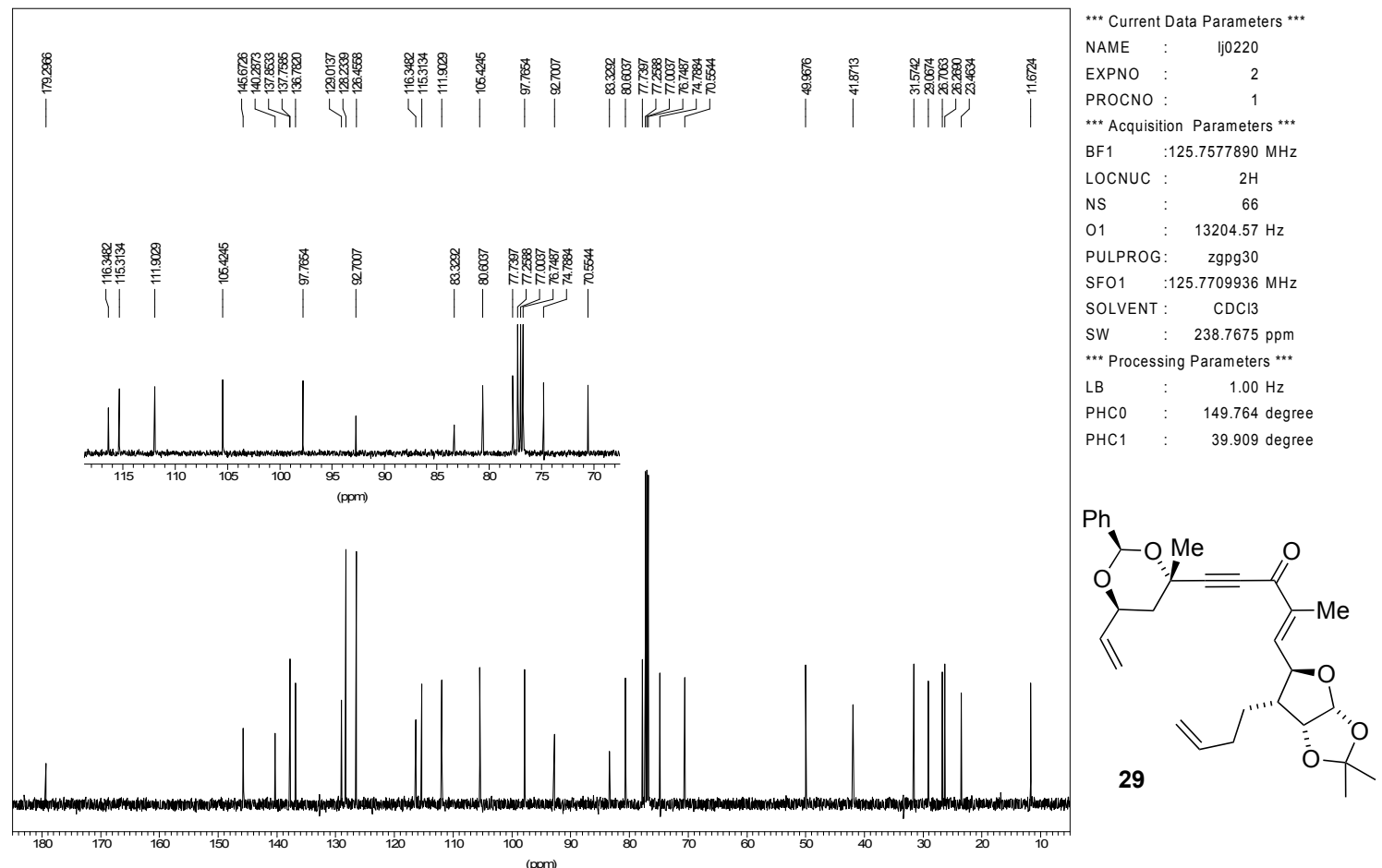
${ }^{1} \mathrm{H}$ NMR (500MHz, $\left.\mathrm{CDCl}_{3}\right)$ and ${ }^{13} \mathrm{C}\{1 \mathrm{H}\}$ NMR $\left(\mathrm{CDCl}_{3}, 125 \mathrm{MHz}\right)$ Spectrums of 30

1H AM X500
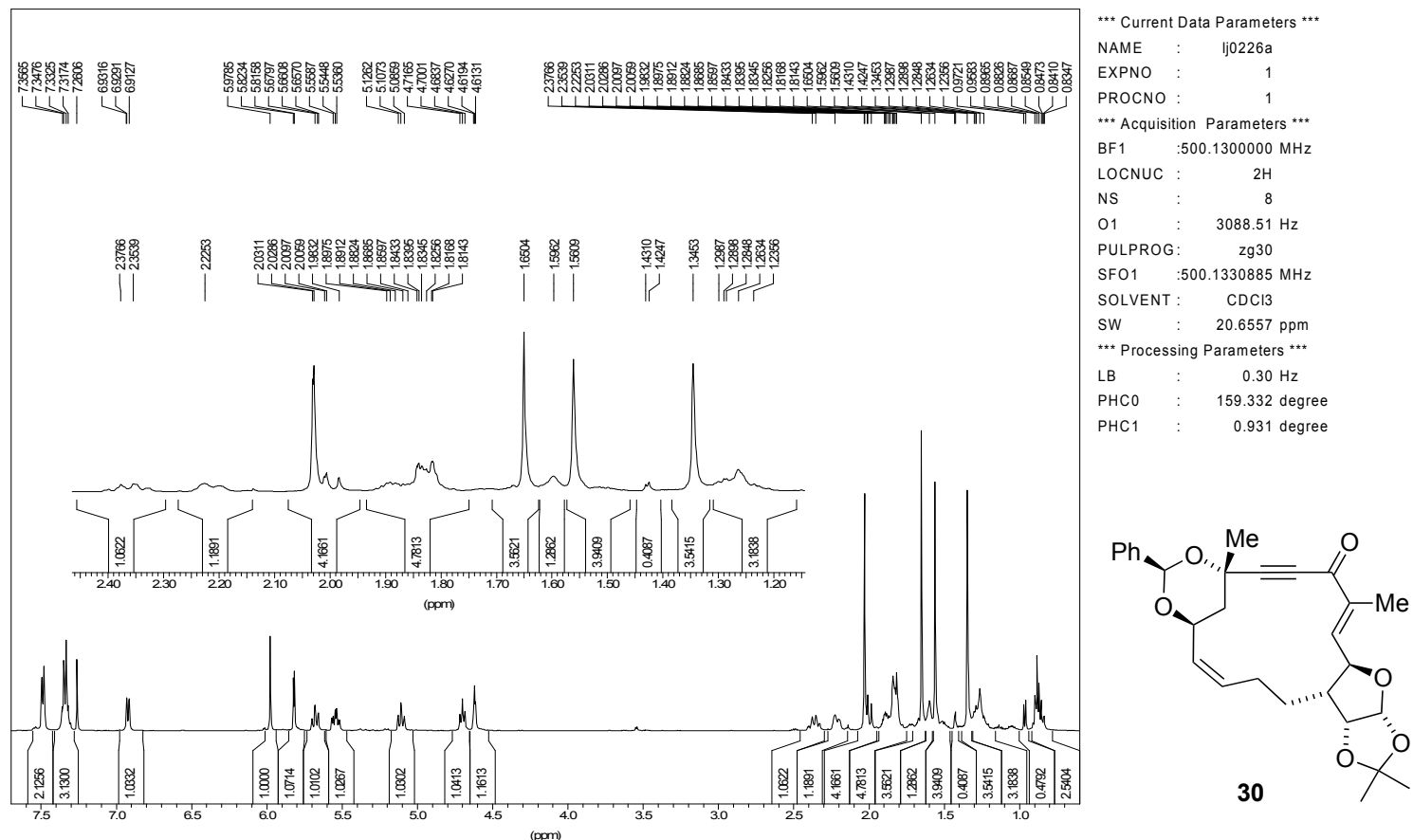

13C AMX500

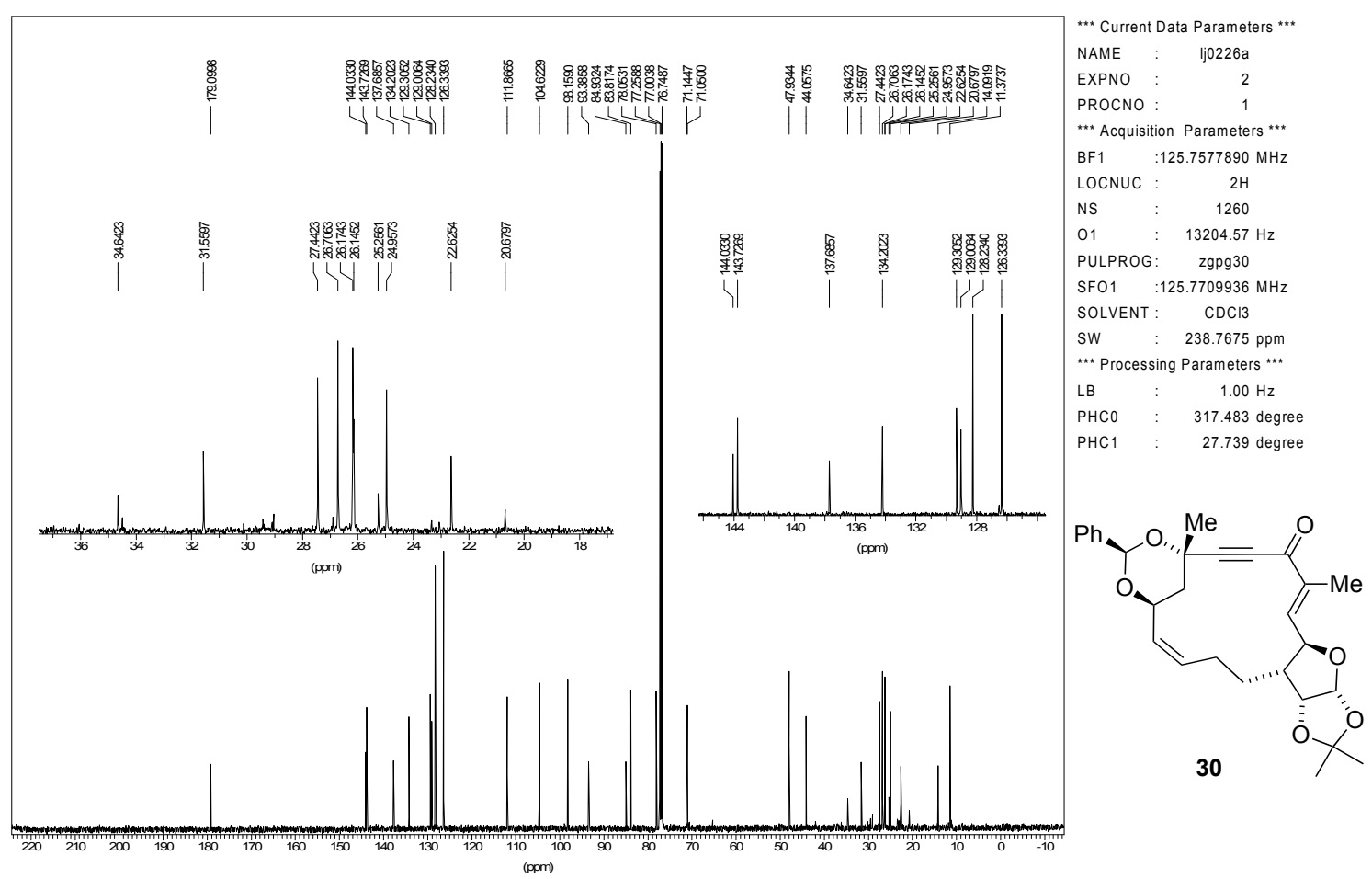


NOESY Spectrum of 30
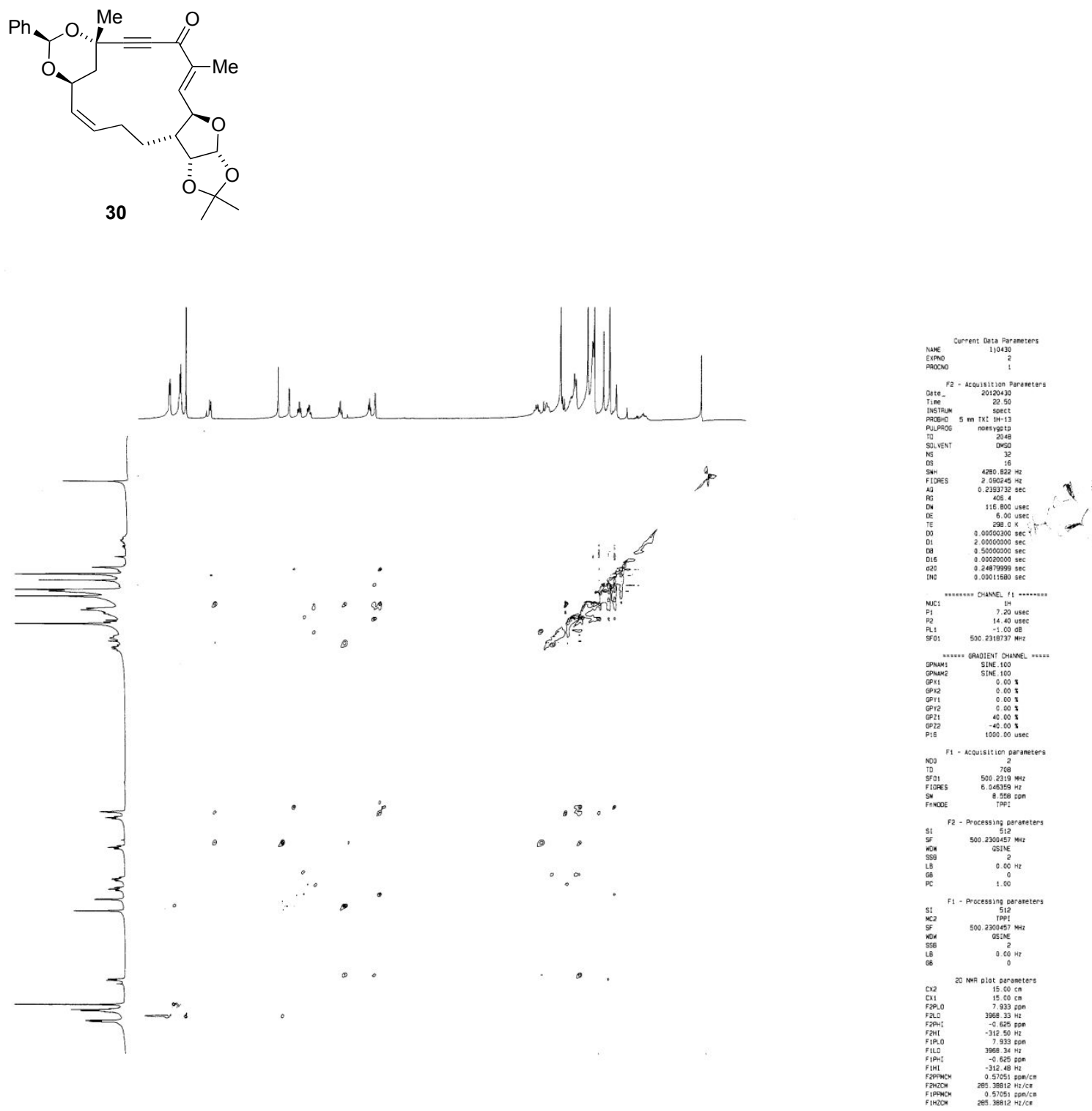